\title{
Horizontal Gene Transfer of Phytochelatin Synthases from Bacteria to Extremophilic Green Algae
}

\section{Olsson, Sanna}

2017-01

Olsson , S , Penacho , V , Puente-Sanchez , F , Diaz , S , Eduardo Gonzalez-Pastor , J \& Aguilera , A 2017 , ' Horizontal Gene Transfer of Phytochelatin Synthases from Bacteria to Extremophilic Green Algae ' , Microbial Ecology , vol. 73 , no. 1 , pp. 50-60 . https://doi.org/10.1007/s00248-016-084

http://hdl.handle.net/10138/307903

https://doi.org/10.1007/s00248-016-0848-z

acceptedVersion

Downloaded from Helda, University of Helsinki institutional repository.

This is an electronic reprint of the original article.

This reprint may differ from the original in pagination and typographic detail.

Please cite the original version. 


\title{
Horizontal gene transfer of phytochelatin synthases from bacteria to
}

\author{
extremophilic green algae
}

\author{
Sanna Olsson ${ }^{1,2 *}$, Vanessa Penacho ${ }^{2,3}$, Fernando Puente-Sánchez ${ }^{2}$, Silvia Díaz ${ }^{4}$, José Eduardo \\ Gonzalez-Pastor ${ }^{2}$ and Angeles Aguilera ${ }^{2}$ \\ ${ }^{1}$ Department of Agricultural Sciences, P.O. Box 27, 00014 University of Helsinki, Finland \\ ${ }^{2}$ Centro de Astrobiología (CSIC-INTA), Carretera de Ajalvir Km 4, Torrejón de Ardoz, 28850 \\ Madrid, Spain \\ ${ }^{3}$ Bioarray, S.L., Parque Científico y Empresarial de la UMH. Edificio Quorum III. Avenida de la \\ Universidad s/n, 03202 Elche, Alicante, Spain \\ ${ }^{4}$ Departamento de Microbiología-III, Facultad de Biología, Universidad Complutense (UCM), \\ Madrid, Spain
}

* Corresponding author: Sanna Olsson, e-mail: sanna.olsson@helsinki.fi, tel.: +358405622456 


\begin{abstract}
Transcriptomic sequencing together with bioinformatic analyses and an automated annotation process led us to identify novel phytochelatin synthase (PCS) genes from two extremophilic green algae (Chlamydomonas acidophila and Dunaliella acidophila). These genes are of intermediate length compared to known PCS genes from eukaryotes and PCS-like genes from prokaryotes. A detailed phylogenetic analysis gives new insight into the complicated evolutionary history of PCS genes and provides evidence for multiple horizontal gene transfer events from bacteria to eukaryotes within the gene family. A separate subgroup containing PCSlike genes within the PCS gene family is not supported since the PCS genes are monophyletic only when the PCS-like genes are included. The presence and functionality of the novel genes in the organisms was verified by genomic sequencing and qRT-PCR. Furthermore, the novel PCS gene in Chlamydomonas acidophila showed very strong induction by cadmium. Cloning and expression of the gene in Escherichia coli clearly improves its cadmium resistance. The gene in Dunaliella was not induced, most likely due to gene duplication.
\end{abstract}

Keywords: heavy metal tolerance, cadmium, acidophile, Chlamydomonas acidophila, Dunaliella acidophila 


\section{INTRODUCTION}

Contamination of soil and water by toxic heavy metals poses a major environmental hazard due to their toxicity and accumulation behavior [1]. Thus, metals are among the most studied polluting agents. Heavy metals are usually toxic because they displace endogenous metal cofactors from their cellular binding sites, undergo aberrant capping reactions with the thiol groups of proteins and thiol containing coenzymes, and promote the formation of active oxygen species [2]. Of the various detoxification pathways activated in plants under heavy metal stress, increased synthesis of sulfur containing defense compounds, namely cysteine, glutathione (GSH) and phytochelatins (PCs), is considered to be of prime importance for their tolerance and survival $[3,4]$. In addition to a variety of plants, their synthesis is also increased in algae and fungi [4-5] upon metal exposure.

Phytochelatins (PCs) mediate high-affinity binding and contribute to detoxification of heavy metal ions and metalloids, such as cadmium or arsenic, by promoting the vacuolysosomal sequestration of heavy metals [6]. These compounds are enzymatically synthesized from reduced glutathione (GSH) and related thiols in a $p$-glutamyl-cysteinyltranspeptidation reaction catalyzed by phytochelatin synthase (PCS). Genes encoding phytochelatin synthases have been found in all vascular plants as well as some algae, fungi, diatoms and invertebrates [4, 7-9]. However, studies regarding PCS gene expression levels have only been examined in a few vascular plants and, in many cases, the results have been contradictory [10-12]. In addition to the eukaryotic full-length phytochelatin synthase genes, PCS-like or half-size PCS proteins were detected initially in prokaryotes (reviewed by Rea et al. [2]). These proteins were described to have a similar $\mathrm{N}$-terminal but lack the more sequence-variable $\mathrm{C}$-terminal, being therefore shorter (220-237 compared to 421-506 amino acid residues). Since then, PCS-like genes have also been reported in some eukaryotes (e.g. fungi: Saccharomyces pombe, Phytophtora infestans, Dictyostelium discoideum; heterokonta: Tetrahymena thermophila; metazoan: Caenorhabditis elegans, Hydra magnipapillata, Strongylocentrotus purpuratus) with scattered distribution but the functionality of these transcripts has largely remained untested [13-14].

Most studies concerning heavy metals and microorganism physiology are related to environments polluted by industrial and domestic wastes. Only little is known about the toxicity of these substances in environments with a natural, non-anthropogenic source of high levels of 
heavy metals, such as extreme acidic ecosystems. Acidic environments tend to contain unusually high concentrations of heavy metals, because their solubility increases markedly as the $\mathrm{pH}$ decreases [15]. Despite these extreme environmental conditions, a large number of prokaryotic and eukaryotic organisms have been identified to live in the presence of high concentrations of heavy metals [16-17]. Acidophiles can survive in $\leq 1000$-fold higher heavy metals amounts than neutrophilic microorganisms [18]. These highly metal resistant organisms seem to have multiple and/or more efficient active resistance systems than neutrophiles [19]. Modern biotechnological methods like high-throughput sequencing of non-model organisms that live in extreme habitats can help to understand the metabolism that allows these organisms to live in their environment and also enable the discovery of novel chemical components.

In a recent study on genes affected by copper excess in a strain of the microalga Chlamydomonas acidophila isolated from Río Tinto (one of the largest extreme acidic environments described until now), transcripts with a putative function related to stress and heavy metal detoxification were identified from a de novo transcriptomic assembly [20]. Several of these transcripts got a best blast hit in other organisms than green algae. One of these, a transcript annotated as a glutathione gamma-glutamyl-cysteinyl-transferase (synonymous to PCS), was hypothesized to represent a novel gene absent in other green algae. A putative PCSlike transcript was later also found in another microalga, in a Dunaliella acidophila strain isolated from the same environment [21]. The present study aims to: 1) Characterize these novel PCS genes by additional sequencing to recover the complete genes. 2) Analyze their protein domains and transmembrane topology. 3) Study their functionality and induction under cadmium exposure. Cadmium was selected because its immense water solubility determines its wide distribution in aquatic systems and, its relatively high mobility in comparison to other metals, makes it a metal of major concern in environmental contamination [22-24]. A phylogenetic analysis was performed in order to uncover relationships between PCS genes in plants, bacteria and green algae and to interpret their evolutionary history.

\section{Material AND Methods}

\section{Algae cultivation and exposure to Cadmium}


Chlamydomonas acidophila strain RT46 was collected from water samples taken in 2010 at the CEM station of Río Tinto (SW Spain) [17], and isolated to grow in the presence of antibiotics, vancomycin $50 \mu \mathrm{g} / \mathrm{mL}$, cefotaxime $100 \mu \mathrm{g} / \mathrm{mL}$ and chloramphenicol $15 \mu \mathrm{g} / \mathrm{mL}$ (Sigma Aldrich, $\mathrm{MO}, \mathrm{US})$, on agar plates made with $0.22 \mu \mathrm{m}$-filtered river water. Clonal cultures were grown in $50-\mathrm{ml}$ flasks in $\mathrm{K}$ medium [25] at pH 2 under an illumination of ca. 70 microE s-1 m-2 irradiance provided by day-light fluorescent tubes, $16: 8 \mathrm{~h}$ LD cycle at 20 ㅇ․

Dunaliella acidophila RT5 was also collected in 2010 at RICHI sampling station, and grown in ARTII medium (developed in our laboratory, see Online Resource 1) at pH 2. When the cultivations reached stationary stage, the cultures were exposed to a Cd solution $\mathrm{CdCl}_{2} 2 \frac{1}{2} \mathrm{H}_{2} \mathrm{O}$ (Panreac Química, Barcelona, Spain). Cultures were maintained during $1 \mathrm{~h}, 3 \mathrm{~h}$ and $24 \mathrm{~h}$ with a final concentration of $1 \mu \mathrm{M}$. Cells were collected in $50 \mathrm{ml}$ Falcon tubes and centrifuged for $5 \mathrm{~min}$ at $5000 \mathrm{rpm}$. Pellets were frozen with liquid nitrogen and stored at $-80^{\circ} \mathrm{C}$ until RNA extraction.

\section{Nucleic acid extraction}

Total RNA was extracted with TRI Reagent ${ }^{\circledR}$ Solution (Thermo Fisher Scientific, MA, US) following manufacturer's protocol. To remove possible DNA contamination the samples were treated with DNase I (RNase free) (Thermo Fisher Scientific). DNA was extracted from cultures not exposed to cadmium using the Wizard $^{\circledR}$ Genomic DNA Purification Kit (Promega Corporation, WI, US) or PurelinkTM Genomic DNA Mini Kit (Thermo Fisher Scientific). DNA and RNA quality were determined spectrometrically $\left(A_{260} / A_{280}>1.8\right)$ using a NanoDrop ND-1000 spectrometer (NanoDrop, DE, US).

\section{Primer design}

Primers for amplification of the full-length genes (CaPCS1, CaPCS2 and DaPCS1) as well as qRT$\mathrm{PCR}$, and cloning and expression of CaPCS2 were designed using the program Primer3 (http://frodo.wi.mit.edu/cgi-bin/primer3/primer3_www.cgi) with default settings. Due to high variability in the PCS transcripts, species specific primers were needed. In quantitative reverse transcription PCR (qRT-PCR) 18S rRNA [26] and actin were both used as housekeeping genes to normalize gene expression. All primers used in this study together with optimal annealing temperatures are listed in Table 1. 


\section{PCR amplification, cloning and sequencing}

PCR amplifications (Bio-Rad Tetrad 2, Bio-Rad, CA, US) of DNA were performed in $25 \mu$ l-reactions following the recommendation for GoTaq ${ }^{\circledR}$ DNA Polymerase (Promega Corporation). PCR settings were as follows: $2 \min 95^{\circ} \mathrm{C}, 35$ cycles $\left(1 \mathrm{~min} 95^{\circ} \mathrm{C}, 1 \mathrm{~min}\right.$ primer specific annealing temperature, $\left.1 \mathrm{~min} 72^{\circ} \mathrm{C}\right), 10 \mathrm{~min} 72^{\circ} \mathrm{C}$. The number of cycles to 40 was frequently used for obtaining stronger products. The PCR products were separated on a $1 \%$ agarose gel and visualized with ethidium bromide using 50 bp DNA ladder (Thermo Fisher Scientific) for size determination. The amplification specificity and efficiency were tested and one primer pair was discarded due to unspecific amplification. The amplified products for each primer pair were cloned with the TOPO TA Cloning kit (Thermo Fisher Scientific) and sequenced on an Applied Biosystems 3730xI DNA Analyzer (Thermo Fisher Scientific) using M13pUC primers and BigDye Terminator v3.1 Cycle Sequencing Kit (Thermo Fisher Scientific). The sequences were edited manually with $\mathrm{PhyDE}^{\circledR}$ v1.0 [27] and primer sequences eliminated before submission to GenBank (accession numbers KT348512 - KT348515).

Occasional non-specific amplification of the target region in Dunaliella acidophila was detected. Strong amplification of the non-specific products was detected using a gradient annealing temperature program $\left(1 \min 95^{\circ} \mathrm{C}, 40\right.$ cycles $\left(1 \min 95^{\circ} \mathrm{C}, 1 \min 53^{\circ} \mathrm{C}-57 \stackrel{\circ}{\circ} \mathrm{C}, 1 \min 72^{\circ} \mathrm{C}, 10\right.$ $\min 72 \stackrel{\circ}{ } \mathrm{C})$.

\section{Quantitative reverse transcription PCR (qRT-PCR)}

For qRT-PCR, protocols established by Díaz et al. [28] were followed, except that the annealing temperature was $55^{\circ} \mathrm{C}$ and actin (ACT1) was used in addition to $18 \mathrm{~S}$ as endogenous control gene. ACT1 primers were modified from the Tetrahymena gene TTHERM_00190950 available on the Tetrahymena Genome Database (TGD) [29]. The cDNA synthesis was carried out using $3 \mu \mathrm{g}$ RNA in $20 \mu$ l-reaction, according to the protocol supplied by 1st Strand cDNA Synthesis kit (AMV, Roche, Basel, Switzerland). All qRT-PCR reactions were carried out in an iQTM5 multicolor RealTime PCR detection System (Bio-Rad) apparatus with the following cycling conditions: (i) 5 min at $95^{\circ} \mathrm{C}$ to denature reverse transcriptase, (ii) 40 cycles of $95^{\circ} \mathrm{C}$ for $30 \mathrm{~s}, 55^{\circ} \mathrm{C}$ for $30 \mathrm{~s}$ and $72^{\circ} \mathrm{C}$ after $20 \mathrm{~s}$. Both NTC (no template control) and RT minus control were negative. The real-time dissociation curve was used to check primer specificity and to confirm the presence of a unique PCR product. Standard curves were obtained using 10 -fold serial cDNA dilutions and 
determining the $\mathrm{Ct}$ (cycle threshold) values. The standard line parameters (amplification efficiency, slope and correlation coefficient) are reported in Online Resource 2. Analysis of relative gene expression was carried out according to the Standard-curve quantification method [30] from at least four independent experiments (each performed in duplicates). Only CaPCS2 was amplified with qRT-PCR, since CaPCS1 was not in the focus of this study and reliable results would not be possible to obtain for DaPCS2 due to assumed gene duplication.

\section{Cloning and expression of the novel PCS gene (CaPCS2) from C. acidophilainE. coli}

The CaPCS2 gene was amplified and cloned in the plasmid pdr111 using the following primers: FQUpHIN and FQLoNHE, which contain the restriction sites HindIII and Nhel required for subsequent subcloning. Plasmid pdr111 was a gift from D. Rudner (Harvard Medical School) and contains the hyper-SPANK promoter, upstream of the cloning site, which is inducible by IPTG. Primer FQUpHIN contains a bacterial ribosomal binding sequence to promote the expression the CaPCS2 gene in E. coli. PCR reaction was carried out using cDNA as DNA template in $20 \mu \mathrm{l}$ reaction containing: 1x PCR buffer 1 (Expand Long Template PCR System, Roche) with $17.5 \mathrm{mM}$ $\mathrm{MgCl} 2,350 \mu \mathrm{M}$ each dNTP, $300 \mathrm{nM}$ each primer, $3.75 \mathrm{U}(0.75 \mu \mathrm{l})$ Expand Long Template enzyme mix (Roche) and $1 \mu$ l of cDNA. Amplification was carried out with the following cycling conditions: $2 \min 94^{\circ} \mathrm{C}, 39$ cycles $\left(1 \min 94^{\circ} \mathrm{C}, 1.5 \min 56^{\circ} \mathrm{C}, 1.5 \min 72^{\circ} \mathrm{C}\right), 10 \min 72^{\circ} \mathrm{C}$.

A unique PCR fragment of approximately $850 \mathrm{bp}$ corresponding to the complete ORF of CaPCS2 gene was gel purified with the QIAquick extraction gel (Qiagen, Hilden, Germany), digested with the restriction endonucleases HindIII (Roche) and Nhel (Roche) and ligated into pdr111 plasmid. Chemically competent Escherichia coli DH5 $\alpha$ were transformed with pdr111 plasmid containing the cloned CaPCS2 gene. Bacterial plasmids were isolated with QIAprep Spin Miniprep kit (Qiagen). The growth medium for transformed bacteria was supplemented with $50 \mu \mathrm{g} \mathrm{ml}-1$ ampicillin to prevent loss of the plasmid in E. coli.

\section{Analysis of Cadmium resistance in $E$. coli clones}

The cadmium resistance was analyzed by the minimum inhibitory concentration (MIC) using serial dilutions 0-10of E. coli clones in LB solid medium (Luria Bertani medium, Laboratorios Conda, Madrid, Spain) and with growth curves by measured the optical density at $600 \mathrm{~nm}$ (OD600). Bacteria carrying the plasmid pdr111 without the gene CaPCS2 cloned were used as 
negative control. The MIC were determinate pipetting $4 \mu$ of each dilution $\left(0-10^{-4}\right)$ onto LB solid medium containing 200-500 $\mu \mathrm{M} \mathrm{CdCl}_{2} 2 \frac{1}{2} \mathrm{H}_{2} \mathrm{O}$ (Panreac Química). The cultures were maintained during $16-24 \mathrm{~h}$ at $37{ }^{\circ} \mathrm{C}$. For the growth curves, OD600 was measured every $60 \mathrm{~min}$ by using a microplate reader (Tecan Genios, Mannedorf, Switzerland) at $37^{\circ} \mathrm{C}$ for 50 cycles ( $49 \mathrm{~h}$ ) as described in Mirete et al. [31]. At least 3 different clones in LB (non-treated) and LB supplemented with 300 or $500 \mu \mathrm{M} \mathrm{CdCl}_{2}$ (treated clones) were analyzed. The pdr111 vector contains a hyper-SPANK promoter, which makes it inducible by IPTG. For this reason, MIC determination and growth curves were carried out in the presence or in the absence of $1 \mathrm{mM}$ IPTG.

\section{Characterization of novel genes}

To form a complete picture of the genes both genomic and transcriptomic sequences were used. Transcripts were detected from de novo assemblies of $C$. acidophila [20] and D. acidophila [21] and in addition the corresponding sequences from another assembly for $C$. acidophila (Olsson et al. unpubl.) were retrieved. Sanger sequencing on genomic DNA was used to complement the information. A flowchart (Online Resource 3) outlines the process from culture to extraction to analyses and shows which assemblies are used in the current study.

\section{Protein characterization}

The nucleotide sequences were translated to amino acids using ExPASy translation tool [32]. The novel predicted proteins were further characterized using the web-server version of HMMER [33] to identify protein domains and the Phobius web-server [34] to predict transmembrane topology and signal peptides.

\section{Identification of similar PCS coding sequences and phylogenetic analyses}

The novel PCS transcripts were used as queries in BLASTx searches [35] against all protein sequences available in GenBank ( $\mathrm{nr}$ database downloaded in April 2013) and the most similar sequences were downloaded. The number of species and different isoforms in closely related species of well represented groups like land plants were limited. Sequences were aligned with Mafft [36] using alignment option FFT-NS-I. The alignment was manually edited in $\mathrm{PhyDE}^{\circledR} \mathrm{V} 1.0$ [27] by excluding ends of the alignments which could not be confidently aligned due to length differences and ambiguities in homology assessment, including the variable C-terminal domain. 
Bayesian analyses were performed with MrBayes v3.2.1 [37], applying search strategies suggested by [37-38]. Calculations of the consensus tree and of the posterior probability of clades were performed based upon the trees sampled after the chains converged. Consensus topologies and support values from the different methodological approaches were compiled and drawn using TreeGraph2 [39].

\section{RESULTS AND DISCUSSION}

\section{Transcripts coding for novel PCS genes}

A full-length PCS from Chlamydomonas acidophila was detected and named as CaPCS1. Transcripts annotated as putative phytochelatin synthases or phytochelatin synthase-like genes in C. acidophila and Dunaliella acidophila were identified as novel genes and named CaPCS2 and DaPCS, respectively. Both of them had best BLAST hits in bacteria, but there are a few eukaryotic sequences among the most similar sequences as well (Blast results are provided in Online Resource 4). The nucleotide sequences have substantial differences between them and only the translated amino acid sequences can be aligned. The genes are illustrated in Figure 1. Additional information on the introns is represented in Online Resource 5.

\section{Characterization of the full-length PCS CaPCS1 from Chlamydomonas acidophila}

The transcript that was similar to known PCS from plants and green alga from C. acidophila, CaPCS1 is 425 amino acids long and contains four introns. Seven different isoforms of the gene were detected. In the predicted 3'UTR (238 bp) two isoforms show a 66 bp long gap, indicating alternative polyadenylation of UTR. Alternative polyadenylation of UTR (APA-UTR) is a widespread phenomenon in eukaryotic cells [40] including Chlamydomonas reinhardtii [41] but has not been documented in C. acidophila.

There are some differences between the transcripts obtained from the different assemblies used in this study. From the de novo assembly by Olsson et al. [20] two different transcripts were obtained of which one (KT348511) is almost identical with the genomic sequence obtained by Sanger sequencing, while the other KT447466 has a unique $58 \mathrm{bp}$ long insertion. Also the sequences from the other transcriptome assembly (Olsson et al. unpubl. corresponding sequences presented in Online resource 6) show several differences, most importantly in the 
5'UTR of two of the sequences (comp17619_c0_seq4 and comp17619_c0_seq6 depicted in Fig.

2). Due to these differences it is hypothesized that two copies of the gene exist. The transcript with the longest open reading frame, which was congruent with the genomic sequence (KT348511), was used for the characterization of the gene.

\section{Characterization of CaPCS2 from Chlamydomonas acidophila}

CaPCS2 consists of four exons and three introns, with a predicted open reading frame of 285 amino acid residues. Alternative polyadenylation of the UTR is present also in this gene; in the eight isoforms covering a 1288 bp long stretch of predicted 5'UTR regions common to all isoforms alter with regions present in only some of the isoforms (Online Resource 7).

A deletion of a nucleotide $235 \mathrm{bp}$ after the beginning of the last exon was detected in the transcript assembled for this study compared to an existing one [20]. Since the deletion occurs in the coding sequence it affects the open reading frame of the gene. The genomic sequence was congruent with the transcript from our previous study (GBAH01012416), which was therefore used for the characterization of the gene for this inconsistent part (depicted in Figure $1)$.

\section{Characterization of DaPCS from Dunaliella acidophila}

Two isoforms of DaPCS1 (comp2951_c3_seq1 and comp2951_c3_seq2) were predicted in the de novo transcriptome assembly of Dunaliella acidophila [21] corresponding accession numbers KT348509-KT348510). One of them has five and the other eight introns (Figure 1).

The nucleotide sequence of contig comp2951_c3_seq1 is longer than comp2951_c3_seq2 but there is an alternative stop codon TAA (instead of CAA coding alanine), which ends the open reading frame. The open reading frame of contig comp2951_c3_seq2 has 390 amino acid residues including a complete phytochelatin synthase domain (pfam 05023) of 293 amino acids as well as a signal peptide and inwards orientated transmembrane domain. Contig comp2951_c3_seq1 has a shorter open reading frame of only 238 amino acids with a truncated phytochelatin synthase domain and neither signal peptides nor transmembrane domains are detected. 
Amplification of DaPCS yielded in addition to the expected amplification product unspecific amplification products, observed by gel electrophoresis. Two different products differing about $650 \mathrm{bp}$ in size were cloned and sequenced (see material and methods and Online Resource 8). The sequences are otherwise identical for the overlapping parts except for two differing nucleotides, suggesting gene duplication in this haploid organism. The sequence of the larger product is congruent with the other genomic and transcriptomic sequences and thus probably represents the prevalent gene copy.

\section{Gene expression of the novel CaPCS2 gene}

Gene expression analysis of the CaPCS2 gene (Chlamydomonas acidophila) was carried out under cadmium treatment using qRT-PCR. The relative mRNA expression levels of target gene were normalized against the levels of actin and 18S rRNA. Statistical analysis for qRT-PCR fold induction indicates that there is a significant difference between the cadmium treatment and the control (Pair Wise Fixed Reallocation Randomisation test, $p<0.01$ ). The gene showed a very strong induction (1275.3 fold induction, \pm 218.8 SD) at three hours, which flatten out at 24 hours (47.8 fold change, \pm 24.1 SD).

As discussed above, it is likely that DaPCS is duplicated, which explains the difficulties involved in the DNA amplification of this region. For this reasons qRT-PCR amplification was not performed on DaPCS. The focus of our study is on the novel PCS genes, and therefore the function of the full-length CaPCS1 gene was not investigated in detail. Furthermore, different isoforms might be differently expressed thus introducing errors to the measurements. The results by Ramos et al. [10] showed that the regulatory mechanisms of PCS expression in plants is very complex including alternative splicing and gene duplication events.

\section{Expression and cadmium resistance of CaPCS2 in E. coli clones}

The cadmium resistance analyzed by the minimum inhibitory concentration (MIC) as explained in material and methods clearly shows that the expression of CaPCS2 gene in E. coli improves their resistance to $\mathrm{Cd}$ (Fig. 3). The effect is observed on LB solid medium with the highest tested concentration $(500 \mu \mathrm{M})$ of $\mathrm{Cd}$. In addition, the growth curves on liquid LB of the strain expressing CaPCS2 in the presence of $\mathrm{Cd}$ confirm this result. The effects of $\mathrm{Cd}$ in a liquid medium are different from solid medium, and the improved resistance to cadmium was better observed 
in LB broth with $300 \mu \mathrm{M}$ added Cd (Fig. 4). In spite of the hyper-SPANK promoter in the used pdr111 plasmid, which controls the expression of the inserted gene CaPCS2 and is inducible by IPTG, addition of IPTG has no significant effect on the cell growth. This could be caused by the transcription of the gene from another constitutively promoter in the plasmid, which dims out the effect of IPTG.

\section{Phylogenetic analyses of phytochelatin synthases}

The results of the phylogenetic analyses show different topology from the expected one based on known species relationships. The only large clade reflecting phylogenetic relationships of organisms is the clade that contains known PCS genes from land plants and green algae. Even within this group some species, such as lotus and Volvox carteri, have different variants or copies of the gene, which were not always monophyletic (Fig. 5). Indeed, many plant species are known to have at least two PCS genes $[10,13]$. The PCS genes from other organisms do not form monophyletic groups congruent with species phylogenies and many of the branches remain unresolved or with low support. Some smaller clades containing bacteria, metazoa or fungi get statistical support but none of them includes all the PCS genes of the group of organisms present in the analysis. Genes from cyanobacteria have a basal position in the phylogenetic tree and do not form a clade.

The full-length PCS (CaPCS1) present in Chlamydomonas acidophila is located in a clade containing PCS from other green algae. No similar transcripts from Dunaliella acidophila or other publicly available Dunaliella sequences were found. This could, however, be due to limited data available for the genus Dunaliella. The novel PCS transcripts (CaPCS2 from Chlamydomonas acidophila and DaPCS1 from Dunaliella acidophila) cluster together but are distinguished by long branches due to very different amino acid sequences between them (Fig. 5and Online Resource 9).

\section{The scattered distribution of PCS genes in nature}

For a long time, researchers have been puzzled by the high constitutive expression of PCS genes in different plant tissues [2]. This is especially peculiar if we assume the presence of high heavy metal concentrations to be an exclusively recent phenomenon caused by human activity like mining. It might be, however, that high heavy metal concentration has been a much more 
severe problem for organisms early in the earth's history and especially arsenic and cadmium comprised a major threat to incipient life forms [42-43]. Indeed, all life forms have evolved strategies to cope with these heavy metals. The best-characterized metalloid resistances are encoded by the ars operons of Gram-negative and Gram-positive bacteria, which are nonspecialized in the way that they share common organization and genes including repressors of resistance to $\mathrm{Zn}(\mathrm{II}), \mathrm{Cd}(\mathrm{II})$ and $\mathrm{Pb}(\mathrm{II})$ (e.g. [44]. If life did emerge in waters rich in ionic metals, resistance to these compounds would probably be a very ancient trait, as suggested by Rosen [45]. Basal plants including mosses and other bryophyte lineages were for a long time supposed not to have PCS genes [13]. Recent results show the opposite, and the presence of constitutively expressed and functional phytochelatin synthases was demonstrated in all the bryophyte lineages by Petraglia et al. [46]. The presence of PCS genes in bryophytes were assayed by western blotting, in vitro activity and by measuring glutathione and phytochelatin contents but the genes have not been sequenced and were therefore not included in our phylogenetic analysis. It is thus not clear if PCSs are key innovations to extreme habitats or ancestral traits, and why they haven't been downregulated or lost through evolution. One plausible explanation would be that PCS serve other physiological functions beyond cadmium or arsenic detoxification, which has been suggested by some authors $[9,14]$.

According to our results PCS genes probably have a bacterial origin and were subsequently inherited to different groups, in some cases multiple times. Bacterial origin is also supported by the domain structure and size of the PCS genes, the bacterial genes being smaller. It has been noted earlier that PCS genes have a non-monophyletic distribution across kingdoms [14] but a comprehensive phylogenetic analyses including PCS genes from both eukaryotes and prokaryotes has not been made earlier. It has been suggested earlier that there might be horizontal gene transfer events involved in the PCS genes in metazoa and fungi [14] and even among vascular plants [10]. Our results confirm that the evolutionary history of these genes is indeed hard to elucidate and show that prokaryotic genes have to be included to resolve their phylogeny. The complex evolution of the PCS genes involves several gene duplications and losses or independent insertions of the full-length PCS genes in plants and green algae.

The great differences in the amino acid composition and sequence length between the novel PCS genes DaPCS and CaPCS2 point to an ancient horizontal gene transfer event, although two 
independent events could also be possible. Horizontal gene transfer (HGT) from bacteria can be beneficial for eukaryotic organisms to survive in adverse conditions [47] and seems to be more common in algae than in vascular plants [48]. Recent studies have emphasized horizontally acquired key genes in adaptation to extreme and rapidly changing environments in other extremophilic algae [49-50]. The novel PCS genes characterized in this study represent a likely example of genes that enable the organism to invade ecological niches otherwise unavailable, in this case an environment toxic for most other organisms.

\section{Conclusions}

Novel PCS genes from two extremophilic green algae are characterized in this study. The results of the phylogenetic analysis oppose the separation of prokaryotic PCS-like genes and suggest multiple horizontal gene transfer events from bacteria to eukaryotes within the PCS gene family. Further functional and structural analyses are needed to understand in detail the role of PCS both in heavy metal detoxification and other possible functions. The gene CaPCS2 increases cadmium tolerance in bacteria but it remains to be seen if the novel genes are transferable to vascular plants since this could be of potential interest for crop science and phytoremediation.

\section{ACKNOWLEDGEMENTS}

This work was supported by the Spanish Ministry of Economy and Competitivity (MINECO) under Grant CGL-2011-22540 to AA. We acknowledge CSC - IT Center for Science Ltd. (Finland) for the allocation of computational resources. Dr. Salvador Mirete (Centro de Astrobiología CSIC-INTA) is acknowledged for assistance with the growth curves for the analysis of cadmium resistance in E. coli. FP-S was supported by a JAE-pre fellowship from the Spanish Consejo Superior de Investigaciones Científicas (CSIC). 


\section{LITERATURE}

1. Jain CK (2004) Metal fractionation study on bed sediments of river Yamuna, India. Water Res 38:569-578

2. Rea PA, Vatamaniuk OK, Rigden DJ (2004) Weeds, Worms, and More. Papain's Long-Lost Cousin, Phytochelatin Synthase. Plant Physiol 136:2463-2474

3. Rausch T, Wachter A. (2005) Sulfur metabolism: a versatile platform for launching defence operations. Trends Plant Sci 10:503-509

4. Grill E, Winnacker E-L, Zenk MH (1987) Phytochelatins, a class of heavy-metal-binding peptides from plants, are functionally analogous to metallothioneins. Proc Natl AcadSci USA 84:439-443

5. Mishra S, Srivastava S, Tripathi RD, Govindarajan R, Kuriakose SV, Prasad MN (2006) Phytochelatin synthesis and response of antioxidants during cadmium stress in Bacopamonnieri L. Plant Physiol Biochem 44:25-3

6. Cobbett CS (2000) Phytochelatin biosynthesis and function in heavy-metal detoxification. Curr Opin Plant Biol 3:211-216

7. Clemens S, Kim EJ, Neumann D, Schroeder JI (1999) Tolerance to toxic metals by a gene family of phytochelatin synthases from plants and yeast. EMBO J 18:3325-3333

8. Vivares D, Arnoux P, Pignol D (2005) A papain-like enzyme at work: native and acylenzyme intermediate structures in phytochelatin synthesis. Proc Natl Acad Sci USA 102:18848-18853

9. Rea PA (2012) Phytochelatin synthase: of a protease a peptide polymerase made. Physiol. Plant 145:154-164 
10. Ramos J, Clemente MR, Naya L et al (2007) Phytochelatin Synthases of the Model Legume Lotus japonicus. A Small Multigene Family with differential response to Cadmium and alternatively spliced variants. Plant Physiol. 143:1110-1118

11. Liu Z, Gu C, Chen F, Yang D, Wu K, Chen S, Jiang J, Zhang Z (2012) Heterologous expression of a Nelumbo nucifera phytochelatin synthase gene enhances cadmium tolerance in Arabidopsis thaliana. Appl Biochem Biotechnol 166:722-734

12. Shukla D, Kesari R, Mishra S et al. (2012) Expression of phytochelatin synthase from aquatic macrophyte Ceratophyllum demersum L. enhances cadmium and arsenic accumulation in tobacco. Plant Cell Rep 31:1687-1699

13. Clemens S (2006) Evolution and function of phytochelatin synthases. J Plant Physiol 163:319-332

14. Clemens S, Peršoh D (2009) Multi-tasking phytochelatin synthases. Plant Science 177:266271

15. Nordstrom DK, Alpers CN (1999) Negative pH, efflorescent mineralogy and consequences from environmental restoration at the Iron Mountain Superfund site, California. Proc Nat Acad Sci USA 96:3455-3462

16. Nixdorf B, Wollmann K, Deneke R (1998) Ecological potentials for planktonic development and food web interactions in extremely acidic mining lakes in Lusatia. In: Geller J, Klapper H Salomons W (eds) Acidic mining lakes. Springer, New York, pp 147-167

17. Aguilera A, Manrubia SC, Gómez F, Rodriguez N, Amils R (2006) Eukaryotic community distribution and their relationship with the water physicochemical parameters in an extreme acidic environment, Río Tinto (SW, Spain). Appl Environ Microbiol 72:5325-5330 
18. Dopson M, Baker-Austin C, Koppineedi PR, Bond P (2003) Growth in sulfidic mineral environments: metal resistance mechanisms in acidophilic micro-organisms. Microbiol 149:1959-1970

19. Dopson M, Ossandon FJ, Lövgren L, Holmes, D (2014) Metal resistance or tolerance? Acidophiles confront high metal loads via both abiotic and biotic mechanisms. Front Microbiol $5: 1-4$

20. Olsson S, Puente-Sánchez F, Gómez-Rodriguez M, Aguilera A (2015) Transcriptional response to copper excess and identification of genes involved in heavy metal tolerance in the extremophilic microalga Chlamydomonas acidophila. Extremophiles 19:657-672

21. Puente-Sánchez F, Olsson S, Aguilera A (2016) Comparative transcriptomic analysis of the response of Dunaliella acidophila (Chlorophyta) to short-term Cadmium and chronic natural metal-rich water exposures. Microb Ecol DOI: 10.1007/s00248-016-0824-7

22. Guanzon NG, Nakahara H, Yoshida Y (1994) Inhibitory effects of heavy metals on growth and photosynthesis of three freshwater microalgae. Fish Sci 60:379-384

23. Worms IA, Boltzman J, Garcia M, Slaveykova VI (2012) Cell-wall-dependent effect of carboxyl-CdSe/ZnS quantum dots on lead and copper availability to green microalgae. Environ Pollut 167:27-33

24. de Abreu FC, da Costa PN, Brondi AM et al (2014) Effects of cadmium and copper biosorption on Chlorella vulgarisBull Environ ContamToxicol 93:405-9

25. Keller MD, Selvin RC, Claus W, Guillard RRL (1987) Media for the culture of oceanic ultraphytoplankton. J Phycol 23:633-638

26. Fei X, Eriksson M, Li Y, Deng X (2010) A Novel Negative Fe-Deficiency-Responsive Element and a TGGCA-Type-Like FeRE Control the Expression of FTR1 in Chlamydomonas reinhardtii. J Biomed Biotechnol 2010:790247 
27. Müller K, Quandt D, Müller J, Neinhuis C (2005) PhyDE ${ }^{\circledR}$ 0.995: Phylogenetic Data Editor, http://www.phyde.de

28. Díaz S, Amaro F, Rico D et al (2007) Tetrahymena metallothioneins fall into two discrete subfamilies. PLoS ONE 2:e291

29. Stover NA, Krieger CJ, Binkley G et al (2005) Tetrahymena Genome Database (TGD): a new genomic resource for Tetrahymena thermophila research. Nucleic Acids Res 34:D500-D503

30. Larinov A, Krause A, Miller W (2005) Standard curve based method for relative real time PCR data processing. BMC Bioinformatics 6:62

31. Mirete S, Mora-Ruiz MR, Lamprecht-Grandío M, de Figueras CG, Rosselló-Móra R, GonzálezPastor JE (2015) Salt resistance genes revealed by functional metagenomics from brines and moderate-salinity rhizosphere within a hypersaline environment. Front Microbiol 6:1121.

32. Artimo P, Jonnalagedda M, Arnold K et al (2012) ExPASy: SIB bioinformatics resource portal. Nucleic Acids Res 40:W597-W603

33. Finn RD, Clements J, Eddy SR (2011) HMMER web server: interactive sequence similarity searching. Nucleic Acids Res 39:W29-W37

34. Käll L, Krogh A, Sonnhammer ELL (2007) Advantages of combined transmembrane topology and signal peptide prediction--the Phobius web server. Nucl Acids Res 35:W429-32

35. Altschul SF, Madden TL, Schaffer AA, Zhang J, Zhang Z, Miller W, Lipman DJ (1997) Gapped BLAST and PSI-BLAST: A new generation of protein database search programs. Nucl Acids Res 25:3389-3402

36. Katoh K, Misawa K, Kuma K, Miyata T (2002) MAFFT: a novel method for rapid multiple sequence alignment based on fast Fourier transform. Nucl Acids Res 30:3059-3066 
37. Ronquist F, Huelsenbeck JP (2003) MRBAYES 3: Bayesian phylogenetic inference under mixed models. Bioinformatics 19:1572-1574

38. Huelsenbeck JP, Ronquist F, Nielsen R, Bollback JP (2001) Bayesian inference of phylogeny and its impact on evolutionary biology. Science 294:2310-2314

39. Stöver BC, Müller, KF (2010) TreeGraph 2: Combining and visualizing evidence from different phylogenetic analyses. BMC Bioinformatics 11:7

40. Di Giammartino DC, Nishida K, Manley JL (2011) Mechanisms and consequences of alternative polyadenylation. Mol Cell 43:853-866

41. Shen Y, Liu Y, Liu L, Liang C, Li QQ (2008) Unique features of nuclear mRNA poly(A) signals and alternative polyadenylation in Chlamydomonas reinhardtii. Genetics 179:167-176

42. Oremland RS, Saltkov CW, Wolfe-Simon F, Stolz JF (2009) Arsenic in the evolution of earth and extraterrestrial ecosystems. Geomicrobiol J 26:522-536

43. Dani SU (2010) Gold, coal and oil. Med Hypotheses 74:534-541

44. Mendoza-Cózatl DG, Jobe TO, Hauser F, Schroeder JI (2011) Long-distance transport, vacuolar sequestration, tolerance, and transcriptional responses induced by cadmium and arsenic. Curr Opin Plant Biol 14:554-562

45. Rosen BP (1999) Families of arsenic transporters. Trends Microbiol 7:207-212

46. Petraglia A, De Benedicti M, Degola F et al (2014) The capability to synthesize phytochelatins and the presence of constitutive and functional phytochelatin synthases are ancestral (plesiomorphic) characters for basal land plants. J Exp Bot 65:1153-1163 
47. Keeling PJ, Palmer JD (2008) Horizontal gene transfer in eukaryotic evolution. Nat Rev Genet 9:605-618

48. Richards TA, Soanes DM, Foster PG, Leonard G, Thornton CR, Tallbot NJ (2009) Phylogenomic analysis demonstrates a pattern of rare and ancient horizontal gene transfer between plants and fungi. Plant Cell 21:1897-1911

49. Blanc G, Agarkova I, Grimwood J, Kuo A, Brueggeman A, Dunigan DD et al (2012) The genome of the polar eukaryotic microalga Coccomyxa subellipsoidea reveals traits of cold adaptation. Genome Biol 13:R39

50. Schönknecht G, Chen WH, Ternes CM et al (2013) Gene transfer from bacteria and archaea facilitated evolution of an extremophilic eukaryote. Science 339:1207-10 


\section{TABLES}

Table 1. Oligonucleotide primers used in the study. For each region, all forward (F) and reverse (R) primers are indicated as well as optimal annealing temperature, target species and references if not designed for this study. Primers used for quantitative reverse transcription PCR are marked with an asterisk *. Primers used for cloning and expression of the novel PCS gene in Chlamydomonas acidophila (CaPCS2) are marked with an exclamation mark!

\section{FIGURE LEGENDS}

Fig. 1 Structures of the predicted genes DaPCS1, CaPCS1 and CaPCS2. Composition of exons (black, numbered E1, E2, etc.) including untranslated regions (gray) and introns (white) of the three genes is given in bp and drawn to scale. An asterisque $\left({ }^{*}\right)$ indicates that only transcriptomic data is available for the UTR

Fig. 2 A partial sequence alignment of the predicted 3'UTR in CaPCS1 from Chlamydomonas acidophila. The sequences from different assemblies (2227_isotig04503 from the assembly by Olsson et al. 2015 and comp17619_c0_seq4 and comp17619_c0_seq6 from a Trinity assembly based on merged data from 454- and Illumina sequencing) show differences in both nucleotide composition and length. Clustal format uses special characters to indicate that all residues in the column are identical (*), conserved (:) or semi-conserved substitutions have been observed (.)

Fig. 3 A) Drop assay using serial dilutions $0-10^{-4}$ of E. coli DH5 a negative control and E. coli $\mathrm{DH} 5 \alpha$ with CaPCS2 gene cloned into pdr111 vector in LB platessupplemented with $50 \mu \mathrm{g} \mathrm{ml}^{-1}$

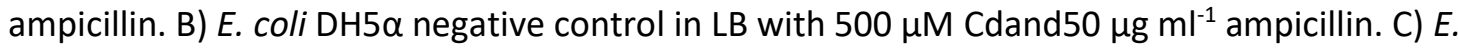
coli with CaPCS2 gene cloned into pdr111 vector in LB with $500 \mu \mathrm{M} \mathrm{Cd}$ and50 $\mu \mathrm{g} \mathrm{ml}^{-1}$ ampicillin.

Fig. 4 Growth curves of Escherichia coli in LB broth supplemented with $300 \mu \mathrm{M}$ of $\mathrm{Cd}$ in presence (+IPTG) or absence (-IPTG) of 1mM IPTG. NC= Escherichia coli negative control. CaPCS2= Escherichia coli cells carrying plasmids with CaPCS2 gene. 
Fig.5 Phylogenetic relationships based on the novel PCS-like genes and the most similar genes obtained with a BLASTX search against NCBI'sdatabases. The tree represents the majority consensus of trees sampled after stationarity in the Bayesian analysis. Posterior probability values from the Bayesian inference equal or greater than 0.50 are shown above each branch. The tree is unrooted. The amino acid alignment was 383 aa long. The kingdoms of each clade are indicated. A miniature phylogram of the consensus tree is depicted to show the branch lengths

\section{ONLINE RESOURCE CAPTIONS}

Online Resource 1 ART II medium used for the cultivation of Dunaliella acidophila strain RT5. The volume was brought to 1 l with distilled water and the $\mathrm{pH}$ adjusted with $0.1 \mathrm{~N} \mathrm{H}_{2} \mathrm{SO}_{4}$.

Online Resource 2 Quantitative reverse transcription PCR standard-curve parameters for target gene (novel phytochelatin synthase in Chlamydomonas acidophila CaPCS2) and the expression control (housekeeping) genes $18 \mathrm{~S}$ rRNA and actin. $\mathrm{E}=$ amplification efficiency, $\mathrm{S}=$ slope, $\mathrm{R}^{2}=$ correlation coefficient.

Online Resource 3 Flow chart on the process from culture to extraction to analyses for the sequences used in the current study.

Online Resource 4Results for BLASTx search using the open reading frame of transcripts coding for CaPCS2 (isotig12422) and DaPCS (comp2951_c3_seq1 and comp2951_c3_seq2) as query.

Online Resource 5 Introns in the predicted PCS genes from C. acidophila and D. acidophila. There are three spliceosomal introns in CaPCS2, four in CaPCS1 and three in DaPCS1. The introns are either the most common GT-AG splice site or variations of it.

Online Resource 6 Different contigs of component comp17619_c0 coding for the CaPCS1 gene in Chlamydomonas acidophila. 
Online Resource 7Different contigs of component comp11852_c0 coding for the CaPCS2 gene in Chlamydomonas acidophila.

Online Resource 8PCR products amplified in Dunaliellaacidophilastrain RT5 using gradient annealing run on $1 \%$ agarose gel (1X TBE). The gel electrophoresis shows amplification of products sized $1198 \mathrm{bp}$ and $542 \mathrm{bp}$ and several non-specific amplification products. The intensity of the non-specific bands depends on the used annealing temperature and PCR program. Wells 1: Ladder $1 \mathrm{~kb}$ (Promega Corporation), marker sizes from $250 \mathrm{~kb}$ to $10000 \mathrm{~kb}$, well2: annealing $52-55^{\circ} \mathrm{C}$. Well 3: annealing $55-60^{\circ} \mathrm{C}$.

Online Resource 9 Phylogram based on the novel phytochelatin synthase genes and the most similar genes obtained with a BLASTx search against NCBI's databases. The tree represents the majority consensus of trees sampled after stationarity in the Bayesian analysis. PP values from the Bayesian inference $>0.50$ are indicated above each branch. The amino acid alignment was 383 aa long. 


\begin{tabular}{|c|c|c|c|c|c|}
\hline Primer name & 5' Sequence 3' & $F / R$ & Ann. ${ }^{\circ} \mathrm{C}$ & Target species & Author \\
\hline CaPCH2_1F & TCACACGAAGGACAAAAGGC & $\mathrm{F}$ & 57 & C. acidophila & This study \\
\hline $\mathrm{CaPCH} 2 \_2 \mathrm{~F}$ & CCAGGGTCATCACTCTCCAA & $\mathrm{F}$ & 53 & C. acidophila & This study \\
\hline CaPCH2_1R & TGCAATTGGAGAGTGATGACC & $\mathrm{R}$ & 57 & C. acidophila & This study \\
\hline $\mathrm{CaPCH} 2 \_3 \mathrm{~F}^{*}$ & TGGGATTGGGATATTGTGCT & $\mathrm{F}$ & 55 & C. acidophila & This study \\
\hline CaPCH2_2R* & ATCTGTTTATGCCCCTGCAC & $R$ & 55 & C. acidophila & This study \\
\hline CaPCH2_3R & AGTGTCATCAAATGATGTTGCAG & $\mathrm{R}$ & 57 & C. acidophila & This study \\
\hline CaPCH1_1F & TGAATACAACAGCGCCACAT & $\mathrm{F}$ & 60 & C. acidophila & This study \\
\hline CaPCH1_1R & GCTCTCCACAAACATGCTCA & $R$ & 60 & C. acidophila & This study \\
\hline CaPCH1_2R & ТССТССССАСТССТАСАСАС & $\mathrm{R}$ & 60 & C. acidophila & This study \\
\hline DaPCH2_1F & TTGTGAAGATGAGGGCATGT & $\mathrm{R}$ & 57 & D. acidophila & This study \\
\hline DaPCH2_2F & ACATCAGGCCCAACATAACG & $\mathrm{F}$ & 57 & D. acidophila & This study \\
\hline DaPCH2_1R & GCTGCGTCTGAACATCTCAA & $R$ & 57 & D. acidophila & This study \\
\hline $\mathrm{DaPCH} 2 \_3 \mathrm{~F}$ & GCTTGTCCCTTGAAGACCTG & $\mathrm{F}$ & 57 & D. acidophila & This study \\
\hline DaPCH2_2R & TGCACGTTACATTCCTGCTC & $R$ & 57 & D. acidophila & This study \\
\hline 18SrRNAF* & TCAACTTTCGATGGTAGGATAGTG & $\mathrm{F}$ & 55 & $\begin{array}{l}\text { D. acidophila, } \\
\text { C. acidophila, } \\
\text { C. reinhardtii }\end{array}$ & Fei et al. 2010 \\
\hline 18SrRNAR* & CCGTGTCAGGATTGGGTAATTT & $R$ & 55 & $\begin{array}{l}\text { D. acidophila, } \\
\text { C. acidophila, } \\
\text { C. reinhardtii }\end{array}$ & Fei et al. 2010 \\
\hline RT46_ACT1* & CTCACTCTCAACATTCCAGCAA & $\mathrm{F}$ & 55 & C. acidophila, & $\begin{array}{l}\text { Modified from } \\
\text { TTHERM_00190950 }\end{array}$ \\
\hline RT46_ACT2* & GGGCCCGCTCTCATCATACTC & $\mathrm{R}$ & 55 & C. acidophila & $\begin{array}{l}\text { Modified from } \\
\text { TTHERM_00190950 }\end{array}$ \\
\hline FQUpHIN! & $\begin{array}{l}\text { TCAGTAAAGCTTAAAGGTGGTGAACTACTATGC } \\
\text { AGGACCCTGCTTCA }\end{array}$ & Left & 58 & C. acidophila & This study \\
\hline FQ_Lo_NHE! & ATCGTAGCTAGCAACAGCGCACAGTGGTGATA & Right & 58 & C. acidophila & This study \\
\hline
\end{tabular}


a)

$$
\text { 5'-UTR E1 E2 E3 E4 E5 E6 E7 E8 E9 }
$$

DaPCs1

\section{$\begin{array}{llllll}92 & 282 & 238 & 444 & 109 & 130\end{array}$}

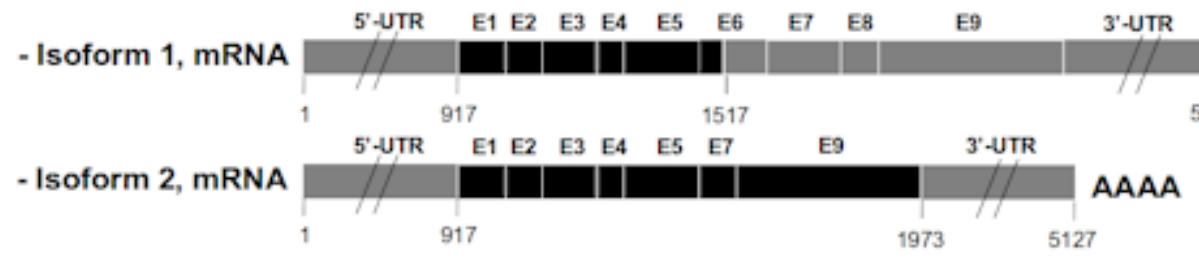

b)

CaPCS1

$5^{\prime}-$ UTR
238
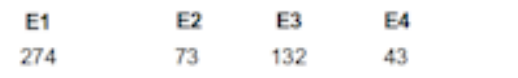

$\mathrm{ES}^{*}$
$753^{*}$

AAAA

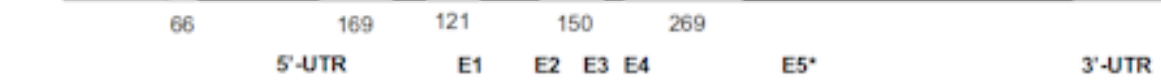

- Isoform 1, mRNA

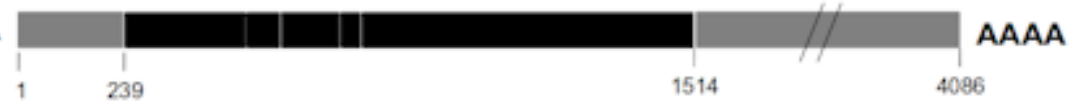

c)

CaPCS2

$274^{5-U T R}$

E1

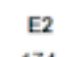

$66 \quad 114$

E3

$\begin{array}{cc}\text { E4 } & 3^{\prime} \text {-UTR } \\ 481 & 5^{*}\end{array}$

- Isoform 1, mRNA
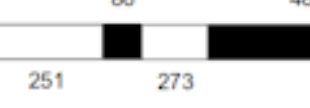

E4 3'-UTR

AAAA 
RT46 2227 Ctg i CATAAACATGGCCTTCAAGAAACTTGCCAGACCAAGGATGACAATGACAATCATTTCACA comp $\overline{1} 7619$ _c0_se CATAAACATGGCCTTCAAGAAACTTGCCAGACCAAGGATGACAATGACAATCATTTCACA comp17619_C0_se CTTAAACATGGCCTTCAAGAACCTTGCCAGACCTAG---GACAATGACACACATTTCACA

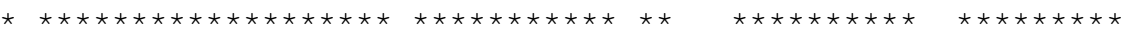

RT46 2227 Ctg i ACGCCCTAAGCAGTCATTTCACGACGCTCTAAGCAGTCGTTGTAAACTTGTAAACAATAC comp $\overline{1} 7619$ _c0_se ACGCCCTAAGCAGTCATTTCACGACGCTCTAAGCAGTCGTTGTAAACTTGTAAACAATAC comp17619 C0 se ACGCCCTAAGCAGTCGTTG---------------------------TAAACAATA-

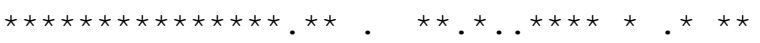

RT46 2227 Ctg i GGCTCTTATTAGTC comp 17619 c0 se GGCTCTTATTAGTC comp17619_c0_se --СTCTTATTACTC 
$10^{0} \quad 10^{-1} \quad 10^{-2} \quad 10^{-3} \quad 10^{-4}$

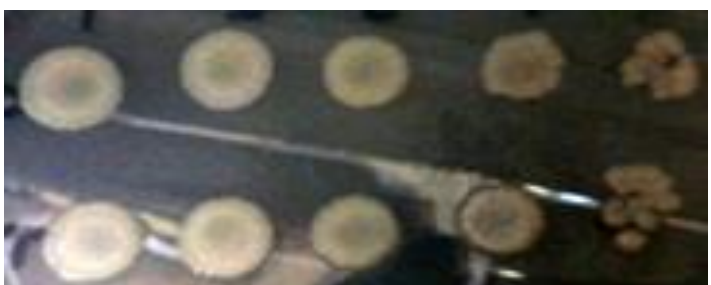

E. coli negative control

E. coli CaPCS2

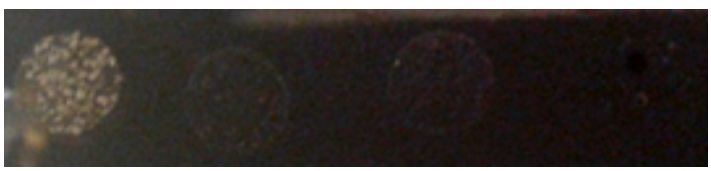

E. coli negative control (500 $\mu \mathrm{M} \mathrm{Cd})$

E. coli CaPCS2 (500 $\mu \mathrm{M}$ Cd) 


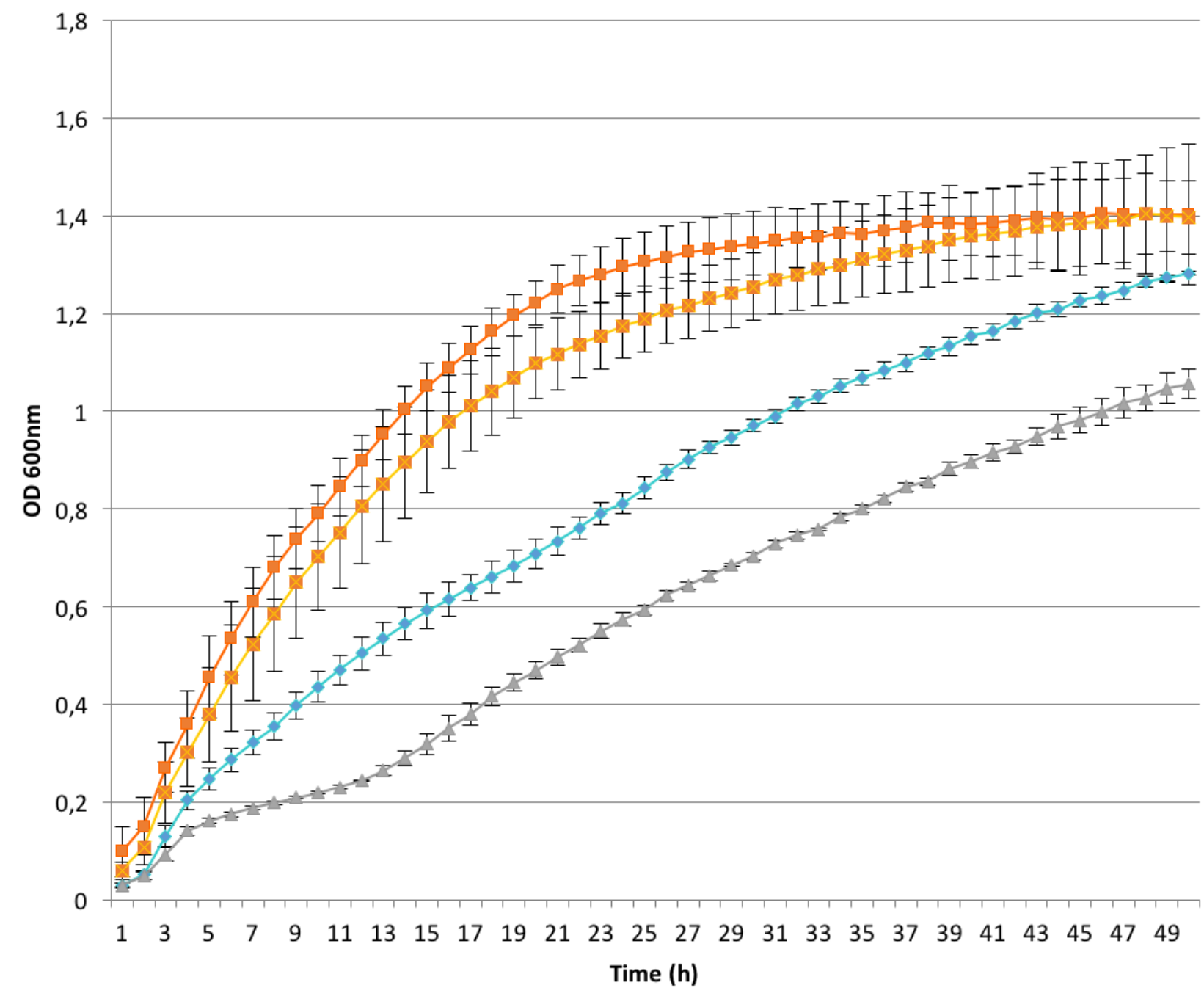

$\longrightarrow$ NC -IPTG

- CaPCS2 - IPTG

$-\mathrm{NC}+$ IPTG

$-\mathrm{CaPCS} 2+$ IPTG

Time (h) 


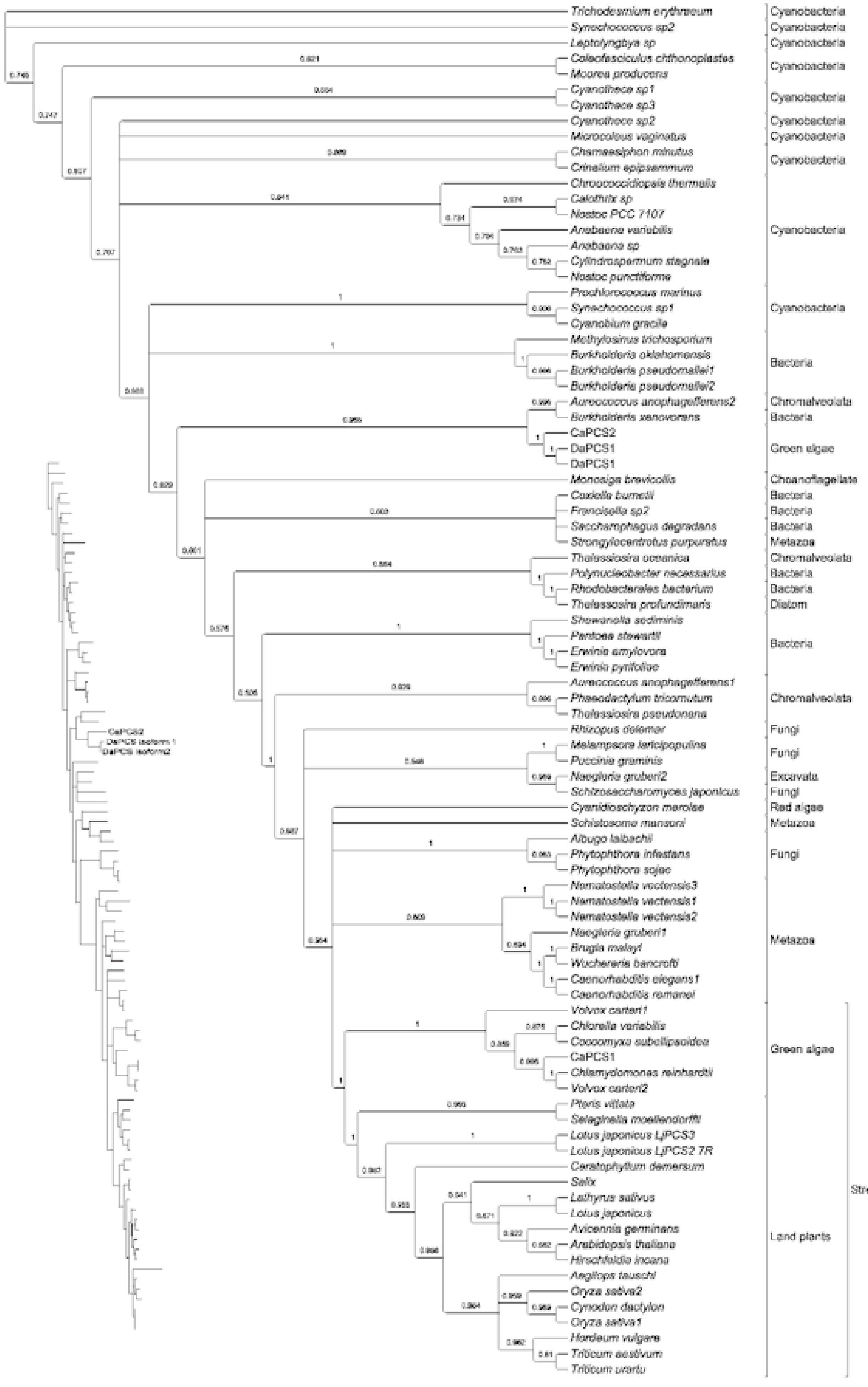

Cyanobecteria

Cyanobuderia

Cyanobacteris

Gyanobecteriat

Cyonobectrerias

Oranobacteria

Chamsasphon mandus

Crinatum eajesemmiton

Caloment $x$ sp

Cylindraspermum stagna

Cyanobium gracilo

Burkholdaris pseudomaleit

- Burkholdurie pseudomelo'?

- Eurkhoideris xenovorens

DaPCS1

Francisolla sp?

Sacchamphagus degradien

Strongyocentrohs purpurgts

Phodobectarales bectarithi

Theilussosiru profundinaris

Chromalvesosta

Func:

Fung:

Streptophyta 
Horizontal gene transfer of phytochelatin synthases from bacteria to extremophilic green algae; Microbial Ecology; Olsson S*, Penacho V, Puente-Sánchez F, Díaz S, Aguilera A; * corresponding author, Department of Agricultural Sciences, P.O. Box 27, 00014 University of Helsinki, Finland, sanna.olsson@ @elsinki.fi

Online Resource 1 ART II medium used for the cultivation of Dunaliella acidophila strain RT5. The volume was brought to 1 l with distilled water and the $\mathrm{pH}$ adjusted with $0.1 \mathrm{~N}_{2} \mathrm{SO}_{4}$.

\begin{tabular}{|c|c|}
\hline Stock Solution & $\mathrm{ml} / \mathrm{l}$ \\
\hline $0.5 \mathrm{M} \mathrm{ZnSO}_{4} \cdot 7 \mathrm{H}_{2} \mathrm{O}$ & $3.5 \mathrm{ml}$ \\
\hline $0.5 \mathrm{M} \mathrm{CuSO}_{4} \cdot 5 \mathrm{H}_{2} \mathrm{O}$ & $8.1 \mathrm{ml}$ \\
\hline $0.5 \mathrm{M} \mathrm{Mn}\left(\mathrm{NO}_{3}\right)_{2} \cdot 6 \mathrm{H}_{2} \mathrm{O}$ & $2.0 \mathrm{ml}$ \\
\hline $0.5 \mathrm{M} \mathrm{Mg} \mathrm{SO}_{4} \cdot 7 \mathrm{H}_{2} \mathrm{O}$ & $27 \mathrm{ml}$ \\
\hline $0.5 \mathrm{M} \mathrm{FeSO}_{4} \cdot 7 \mathrm{H}_{2} \mathrm{O}$ & $30 \mathrm{ml}$ \\
\hline $0.5 \mathrm{M} \mathrm{NiSO}_{4} \cdot 6 \mathrm{H}_{2} \mathrm{O}$ & $80 \mu \mathrm{l}$ \\
\hline $0.5 \mathrm{M} \mathrm{KCl} \cdot 7 \mathrm{H}_{2} \mathrm{O}$ & $380 \mu \mathrm{l}$ \\
\hline $0.5 \mathrm{M} \mathrm{CoCl}_{2} \cdot 6 \mathrm{H}_{2} \mathrm{O}$ & $190 \mu \mathrm{l}$ \\
\hline $0.5 \mathrm{M} \mathrm{KBr} \cdot 5 \mathrm{H}_{2} \mathrm{O}$ & $30 \mu \mathrm{l}$ \\
\hline
\end{tabular}


Horizontal gene transfer of phytochelatin synthases from bacteria to extremophilic green algae; Microbial Ecology; Olsson S*, Penacho V, Puente-Sánchez F, Díaz S, Aguilera A; * corresponding author, Department of Agricultural Sciences, P.O. Box 27, 00014 University of Helsinki, Finland, sanna.olsson@helsinki.fi

Online Resource 2 Quantitative reverse transcription PCR standard-curve parameters for target gene (novel phytochelatin synthase in Chlamydomonas acidophila CaPCS2) and the expression control (housekeeping) genes 18S rNA and actin. $E=$ amplification efficiency, $\mathrm{S}=$ slope, $\mathrm{R} 2=$ correlation coefficient.

\begin{tabular}{|l|l|l|l|}
\hline Gene & E & S & $\mathbf{R}^{2}$ \\
\hline PCS & 1,95 & $-3,43$ & 0,99 \\
\hline 18 S rRNA & 2,09 & $-3,12$ & 0,98 \\
\hline Actin & 2,10 & $-3,2$ & 0,98 \\
\hline
\end{tabular}


Horizontal gene transfer of phytochelatin synthases from bacteria to extremophilic green algae; Microbial Ecology; Olsson S*, Penacho V, Puente-Sánchez F, Díaz S, Aguilera A; * corresponding author, Department of Agricultural Sciences, P.O. Box 27, 00014 University of Helsinki, Finland, sanna.olsson@helsinki.fi

Online Resource 3 Flow chart on the process from culture to extraction to analyses for the sequences used in the current study. 


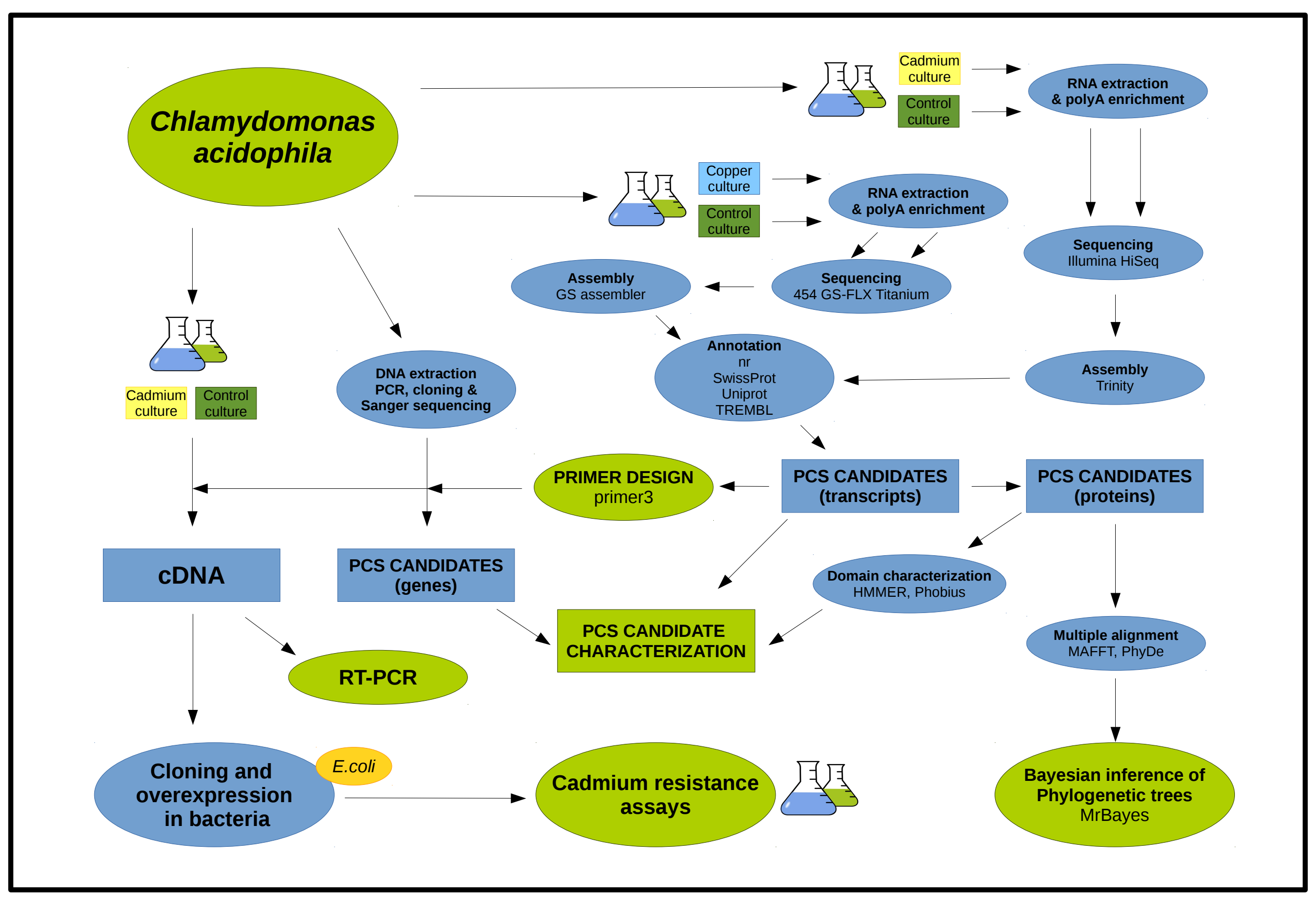


Electronic Supplementary Material 4. Results for BLASTx search using the open reading frame of transcripts coding for CaPCS2 (isotig12422) and DaPCS (comp2951_c3_seq1 and comp2951_c3_seq2) as query.

ORF_isotig12422 $\quad$ gi|821561551|ref|WP_046868556.1| $48.98 \quad 147 \quad 70$

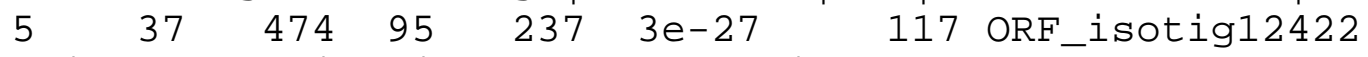
gi|504945810|ref|WP_015132912.1| $42.67 \quad 0 \quad 150 \quad 80 \quad 4 \quad 31 \quad 474 \quad 96$ 241 2e-25 112 ORF_isotig12422 gi|981461217|ref|WP_059669889.1|

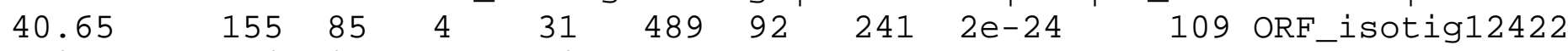

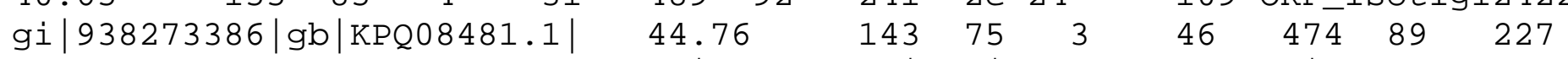
3e-24 108 ORF_isotig12422 gi|981383968|ref|WP_059597148.1| 41.10

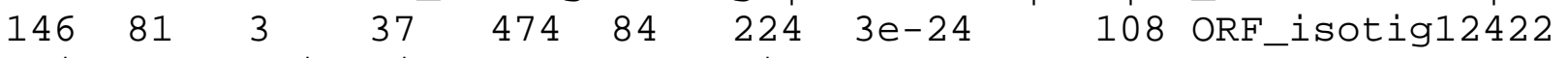
gi|981251097|ref|WP_059470554.1| $41.10 \quad 146 \quad 81^{-1} \quad 3 \quad 37 \quad 474 \quad 93$ $2334 \mathrm{e}-24$ 108 ORF_isotig12422 gi|981645748|ref|WP_059844321.1|

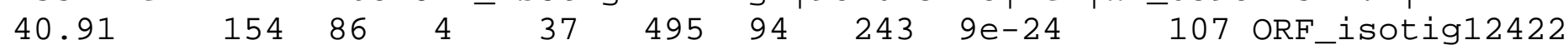
gi|705475049|gb|KGS04910.1| $40.52 \quad \begin{array}{lllllll}153 & 84 & 4 & 37 & 489 & 83 & 230\end{array}$ 1e-23 107 ORF_isotig12422 gi|740960548|ref|WP_038745121.1| 40.52

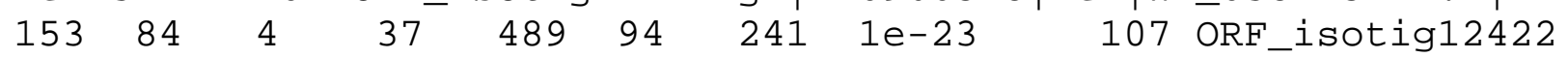
gi|782673738|ref|WP_045600604.1| $40.52 \quad 153 \quad 88 \quad 3 \quad 37 \quad 495 \quad 94$ $2431 \mathrm{e}-23 \quad 107$ ORF_isotig12422 gi|497590677|ref|WP_009904861.1|

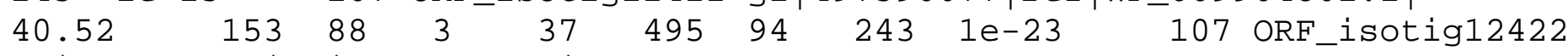

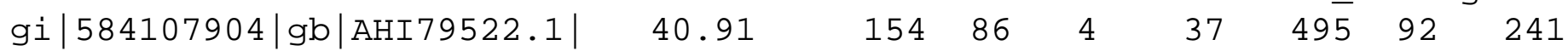
1e-23 107 ORF_isotig12422 gi|497576287|ref|WP_009890471.1| 40.91

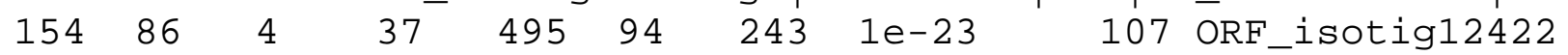
gi|981433289|ref|WP_059643234.1| $40.52 \quad 0153 \quad 84 \quad 4 \quad 37 \quad 489 \quad 94$ 241 1e-23 107 ORF_isotig12422 gi|773064690|gb|AJY42591.1| 41.22

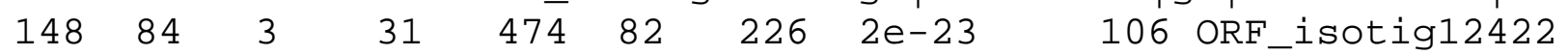

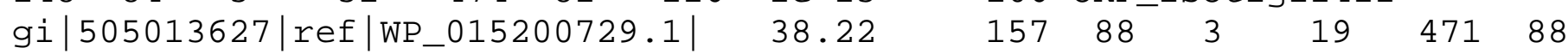
241 2e-23 106 ORF_isotig12422 gi|976471317|gb|KVD74754.1| 39.73

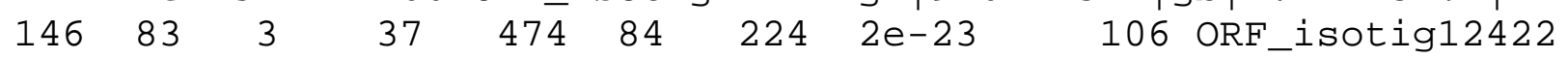
gi|492895672|ref|WP_006026078.1| $39.35 \quad 155 \quad 89 \quad 3 \quad 31 \quad 495 \quad 92$ 241 2e-23 106 ORF_isotig12422 gi|705585042|gb|KGS47698.1| 41.10

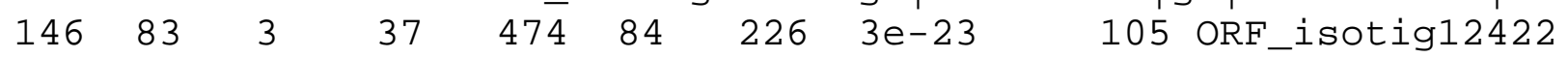

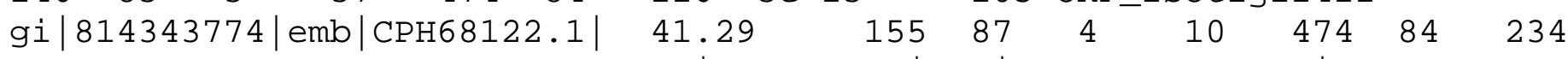
3e-23 105 ORF_isotig12422 gi|981296166|ref|WP_059513522.1| 42.00

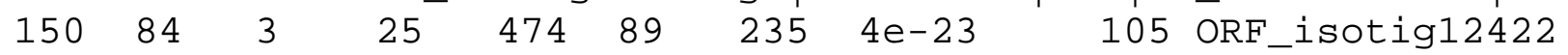

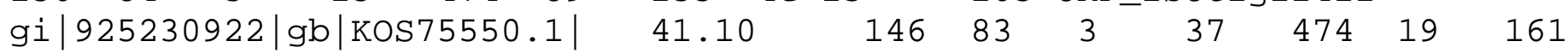
$\begin{array}{llllll}4 \mathrm{e}-23 & 103 & \mathrm{ORF}_{-} \text {isotig12422 } \mathrm{gi}|686848709| \mathrm{gb}|\mathrm{KGC} 28119.1| & 41.22 & 148\end{array}$

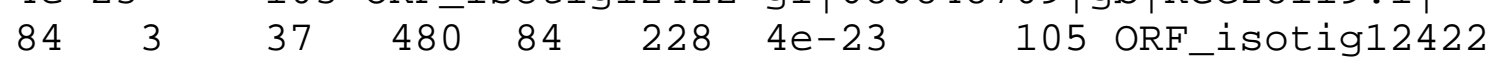

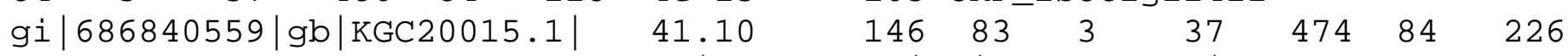
4e-23 105 ORF_isotig12422 gi|976553572|gb|KVE52227.1| $39.73 \quad 146$

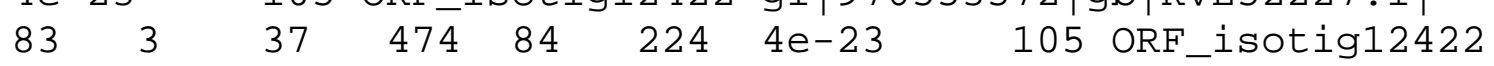

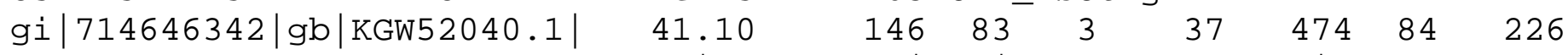
4e-23 105 ORF_isotig12422 gi|741015566|ref|WP_038797782.1| 41.10

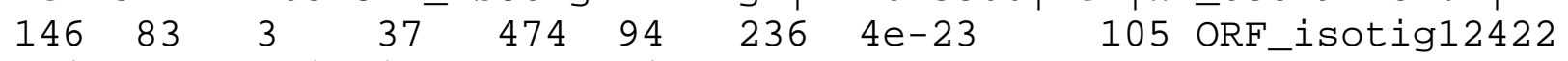

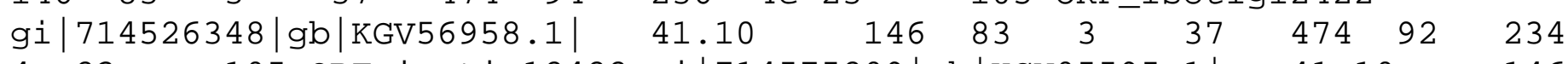
4e-23 105 ORF_isotig12422 gi|714575209|gb|KGW05595.1| $41.10 \quad 146$

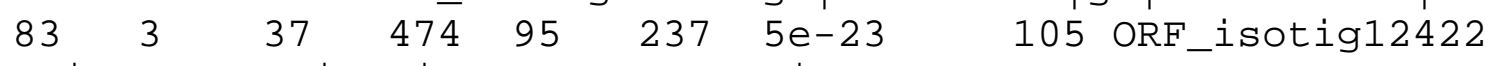
gi|648412694|ref|WP_026104445.1| $43.62 \quad 149 \quad 78 \quad 5 \quad 54 \quad 474 \quad 99$ 243 5e-23 105 ORF_isotig12422 gi|497606907|ref|WP_009921091.1|

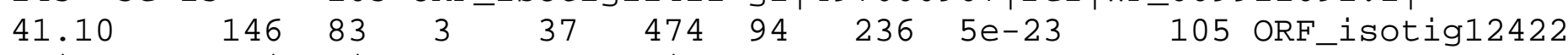
gi|740942487|ref |WP_038727502.1| $41.10 \quad 146 \quad 83 \quad 3 \quad r \begin{array}{lllll}37 & 474 & 97\end{array}$ $2395 e-23 \quad 105$ ORF_isotig12422 gi|740984489|ref|WP_038768164.1|

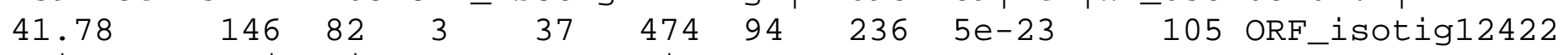
gi|740980945|ref|WP_038764816.1| $41.22 \quad 148 \quad 84 \quad 3 \quad \begin{array}{lllll}37 & 480 & 97\end{array}$ $2416 e-23 \quad 105$ ORF_isotig12422 gi|752526330|ref|WP_041197894.1|

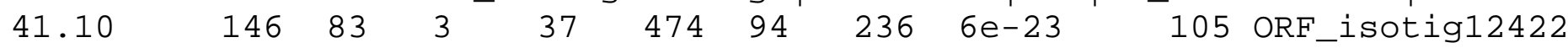


gi |686936961 |gb|KGD15055.1|

41.10

14683

37

$\begin{array}{lll}474 & 52 & 194\end{array}$

6e-23 104 ORF_isotig12422 $\begin{array}{llllll}146 & 83 & 3 & 37 & 474 & 94\end{array}$ gi|740959588|ref|WP_038744210.1| 41.10 gi |714507186 | gb | KGV37859.1| $2366 \mathrm{e}-23$

105 ORF_isotig12422

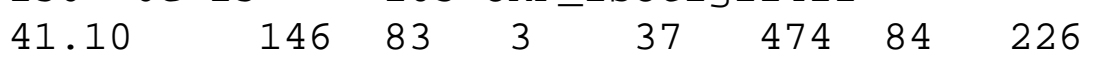
6e-23 105 ORF_isotig12422 gi|490304232|ref|WP_004199532.1| 41.10 $\begin{array}{llllllll}146 & 83 & 3 & 37 & 474 & 84 & 226 & 7 \mathrm{e}-23\end{array}$ 104 ORF_isotig12422

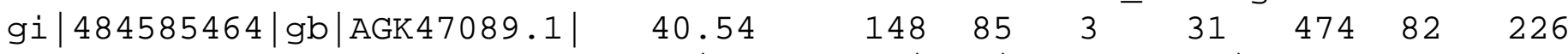
7e-23 104 ORF_isotig12422 gi|814476642|emb|CPI32267.1| $41.10 \quad 146$

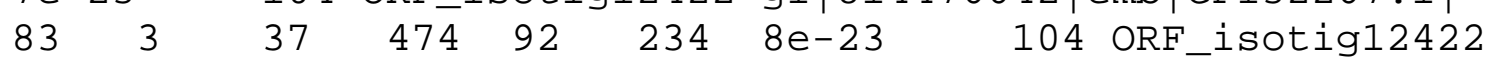
gi|752524486|ref|WP_041196068.1| $41.10 \quad 146 \quad 83 \quad 3 \quad 37 \quad 474 \quad 94$ 236 9e-23 104 ORF_isotig12422 gi|714591842|gb|KGW22095.1| 41.10

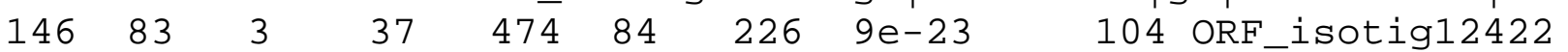
gi|754360430|ref|WP_041861630.1| $40.54 \quad 148 \quad 85 \quad 3 \quad 31 \quad 474 \quad 92$ $2369 \mathrm{e}-23 \quad 104$ ORF_isotig12422 gi|490685647|ref|WP_004550267.1|

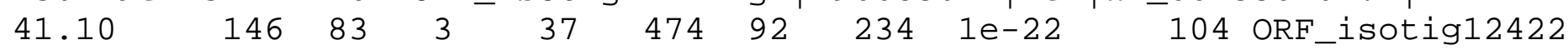
gi|913356948|ref|WP_050376444.1| $41.10 \quad 146 \quad 83 \quad 3 \quad 37 \quad 474 \quad 94$ 236 1e-22 104 ORF_isotig12422 gi|800785064|emb|CFL25926.1| 41.10

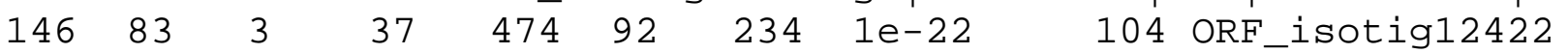
gi|953804284|ref|WP_058039816.1| $41.10 \quad 146 \quad 83 \quad 3 \quad 37 \quad 474 \quad 94$ 236 1e-22 104 ORF_isotig12422 gi|490301683|ref|WP_004197081.1|

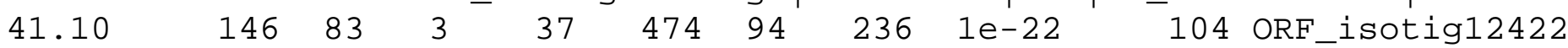
gi|953803876|ref|WP_058039691.1| $41.10 \quad 146 \quad 83 \quad 3 \quad 37 \quad 474 \quad 94$ 236 1e-22 104 ORF_isotig12422 gi|981741072|ref|WP_059934588.1|

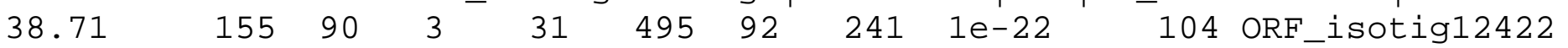
gi|981354997|ref|WP_059570263.1| $39.61 \quad 154 \quad 88 \quad 3 \quad 37 \quad 498 \quad 94$ $2421 \mathrm{e}-22 \quad 104$ ORF_isotig12422 gi|751301901|ref|WP_041010414.1|

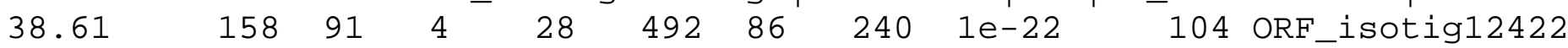
gi|740967447|ref|WP_038751917.1| $41.10 \quad 146 \quad 83 \quad 3 \quad 137 \begin{array}{lllll}94 & 94\end{array}$ 236 1e-22 104 ORF_isotig12422 gi|751277775|ref|WP_040986624.1|

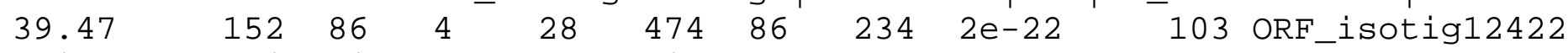
gi|751301199|ref|WP_041009719.1| $38.61 \quad 158 \quad 91 \quad 4 \quad 28 \quad 492 \quad 86$ 240 2e-22 103 ORF_isotig12422 gi|913438795|ref|WP_050418395.1|

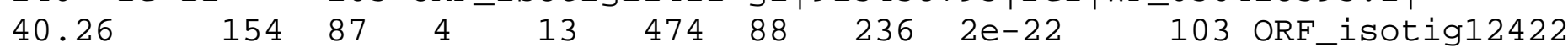
gi|909830227|gb|AKU13025.1| $40.26 \quad 154 \quad 87 \quad 4 \quad 13 \quad 474 \quad 93 \quad 241$ 2e-22 103 ORF_isotig12422 gi|806494169|gb|KKC16091.1| $40.41 \quad 146$

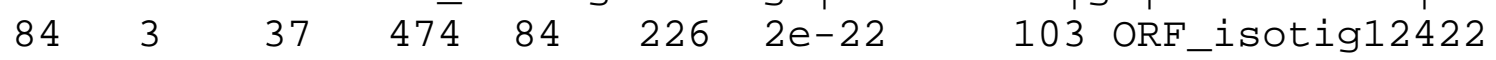

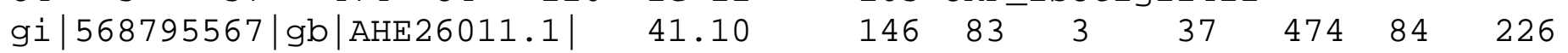
3e-22 103 ORF_isotig12422 gi|772972989|gb|AJX33047.1| $39.73 \quad 146$

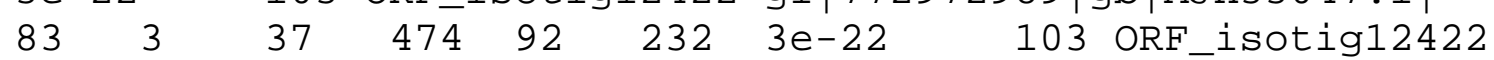
gi|756829253|ref|WP_042646352.1| $39.47 \quad 15 \overline{2} \quad 86 \quad 4 \quad 28 \quad 474 \quad 86$ 234 3e-22 103 ORF_isotig12422 gi|741019975|ref|WP_038801977.1|

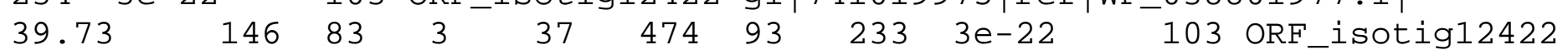
gi|740943421|ref|WP_038728427.1| $40.41 \quad 146 \quad 84 \quad 3 \quad 37 \begin{array}{lllll} & 474\end{array}$ $2364 \mathrm{e}-22$ 103 ORF_isotig12422 gi|740986897|ref|WP_038770440.1|

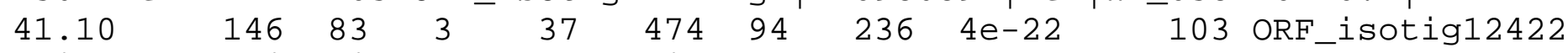
gi|785594494|ref|WP_045719475.1| $40.41 \quad 146 \quad 84 \quad 3 \quad 37 \quad 474 \quad 94$ $2364 \mathrm{e}-22 \quad 102$ ORF_isotig12422 gi|741007236|ref|WP_038789710.1|

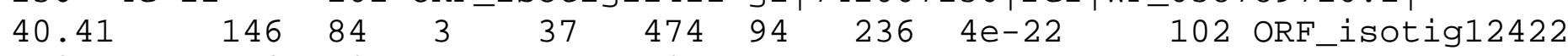
gi|493029431|ref|WP_006099075.1| $42.36 \quad 144 \quad 77 \quad 5 \quad 46 \quad 474 \quad 104$ 242 4e-22 102 ORF_isotig12422 gi|237502694|gb|ACQ95012.1| 41.10

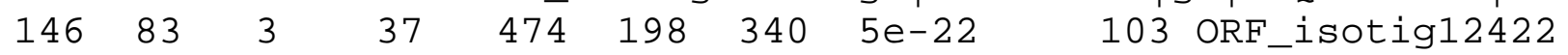

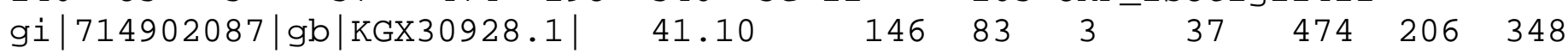
5e-22 103 ORF_isotig12422 gi|157806771|gb|ED083941.1| $41.10 \quad 146$

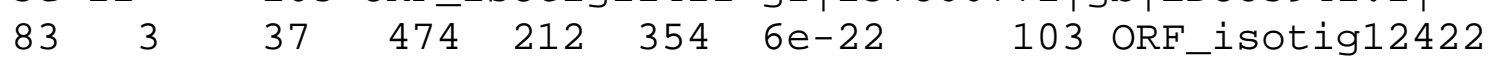

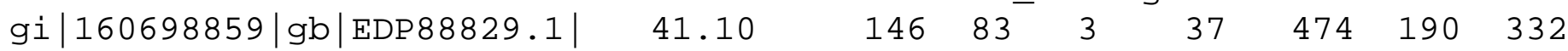
7e-22 103 ORF_isotig12422 gi|689678301|emb|CEF48871.1| $38.36 \quad 146$

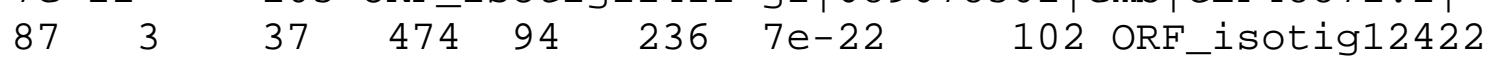


gi | 148027253 | gb|EDK85274 .1|

41.10

$\begin{array}{llll}146 & 83 & 3 & 37\end{array}$

$474 \quad 194$

336

7e-22 103 ORF_isotig12422

gi $|243063500| \mathrm{gb}|\mathrm{EES} 45686.1|$

41.10

146

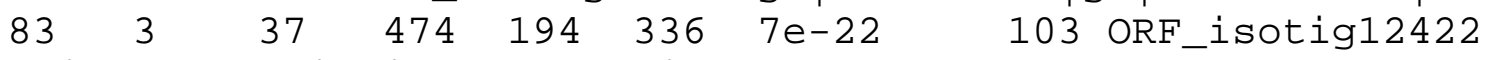

gi | 121227363 | gb | ABM49881 . 1 |

41.10

$\begin{array}{llll}146 & 83 & 3 & 37\end{array}$

$474 \quad 196$

338

7e-22 103 ORF_isotig12422 gi|126229014|gb|ABN92554.1|

$\begin{array}{lllllll}83 & 3 & 37 & 474 & 199 & 341 & 7 \mathrm{e}-22\end{array}$

103 ORF_isotig12422

gi|685679385|gb|AI066505.1|

39.73

$146 \quad 83 \quad 3 \quad 37$

41.10

146

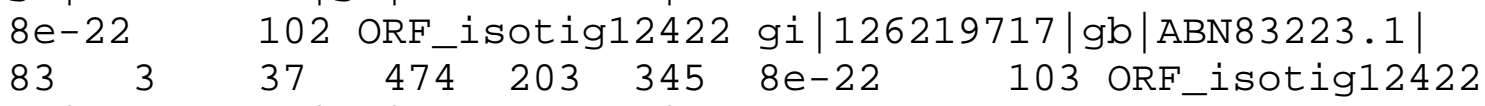

gi|169654349|gb|EDS87042.1| $41.10 \quad 146 \quad 83 \quad 3 \quad 37$

$\begin{array}{lll}474 & 92 & 232\end{array}$

$41.10 \quad 146$

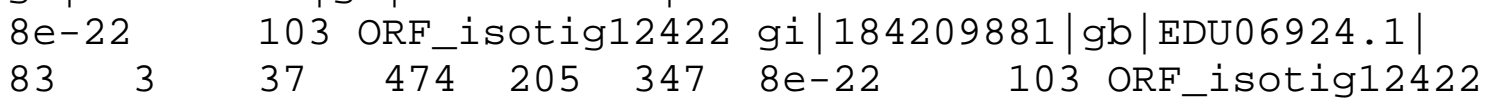

$\begin{array}{lllllllll}83 & 3 & 37 & 474 & 205 & 347 & 8 \mathrm{e}-22 & 103 & \text { ORF_isotig12422 }\end{array}$

$474 \quad 209 \quad 351$

$41.10 \quad 146$

233 8e-22 102 ORF_isotig12422 gi|157936951|gb|ED092621.1

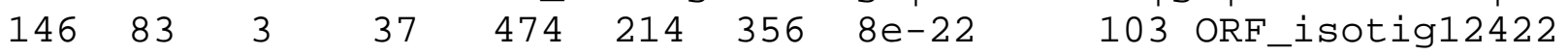

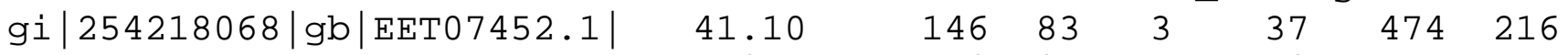

8e-22 103 ORF

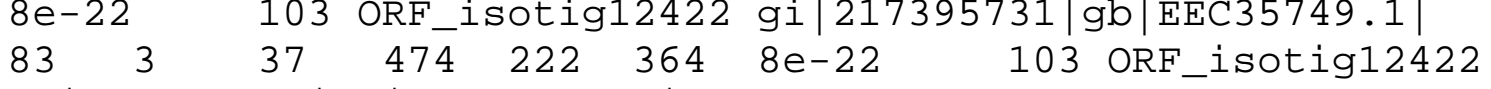

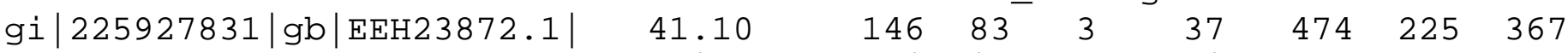

8e-22 103 ORF_isotig12422 gi|134251017|gb|EBA51096.1| $41.10 \quad 146$

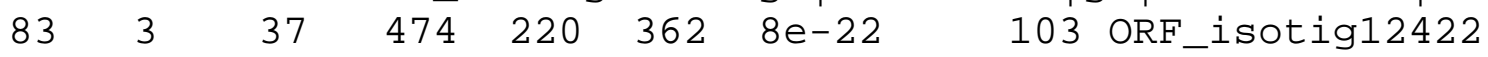

gi|501733845|ref|WP_012629148.1| $41.18 \quad 153 \quad 79 \quad 5 \quad 31 \quad 474 \quad 95$

241 8e-22 102 ORF_isotig12422 gi|927917924|emb|CDX53272.1| 39.47

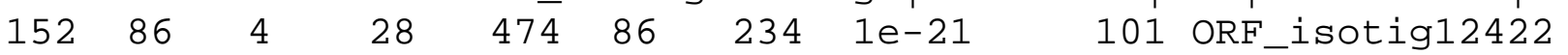

gi|499931312|ref|WP_011612046.1| $42.36 \quad 144 \quad 79 \quad 3 \quad 43 \quad 474 \quad 102$

241 2e-21 100 ORF_isotig12422 gi|503088795|ref|WP_013323642.1|

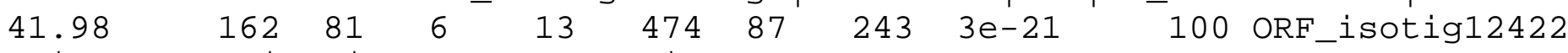

gi|826018244|ref|WP_-047165229.1| $41.67 \quad 144 \quad 80 \quad 3 \quad 43 \quad 474 \quad 90$

229 4e-21 100 ORF_isotig12422 gi|504949901|ref|WP_015137003.1|

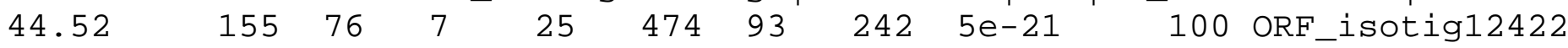

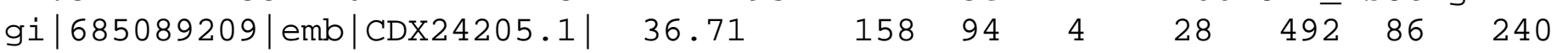

7e-21 99.4 ORF_isotig12422 gi|751264288|ref|WP_040974001.1|

$\begin{array}{llllllllll}38.16 & 152 & 88 & 4 & 28 & 474 & 88 & 236 & 8 \mathrm{e}-21 & 99.4\end{array}$

ORF_isotig12422 gi|966518160|ref|WP_058534654.1| $39.51 \quad 162 \quad 85$

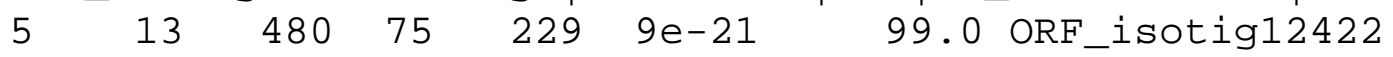

gi|974600092|ref|WP_059190108.1| $36.71 \quad-158 \quad 94 \quad 4 \quad 28 \quad 492 \quad 86$

$2401 \mathrm{e}-20 \quad 98.6$ ORF_isotig12422 gi|764950513|ref|WP_044520790.1|

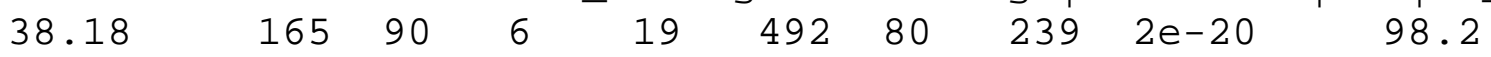

$\begin{array}{llllll}\text { ORF_isotig12422 } & \text { gi|17130321|dbj|BAB72932.1| } & 38.18 & 165 & 90 & 6\end{array}$

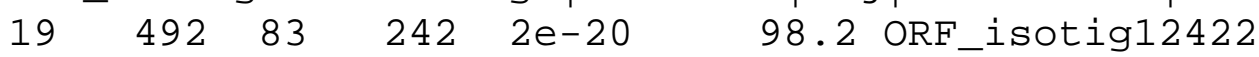

gi|652914138|ref|WP_027168079.1| $38.82 \quad 152 \quad 87 \quad 4 \quad 28 \quad 474 \quad 86$

234 2e-20 97.8 ORF_isotig12422 gi|701274860|gb|AIV79086.1|

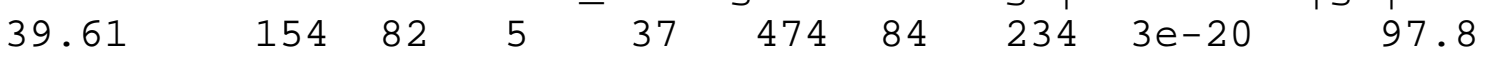

ORF_isotig12422 $\quad$ gi|75702893|gb|ABA22569.1| $38.36 \quad 159 \quad 87 \quad 5$

$19 \quad 474 \quad 80 \quad 234 \quad 3 e-20 \quad 97.4$ ORF_isotig12422

gi|759580794|ref|WP_043299684.1| $39.61 \quad 34 \quad 82 \quad 5 \quad 37 \quad 474 \quad 94$

244 4e-20 97.4 ORF_isotig12422 gi|657935844|ref|WP_029636980.1|

$\begin{array}{llllllllll}34.81 & 158 & 94 & 3 & 19 & 474 & 88 & 242 & 4 \mathrm{e}-20 & 97.4\end{array}$

ORF_isotig12422 gi|496440563|ref|WP_009149408.1| $39.33 \quad 150 \quad 85$

$4 \quad 31 \quad 474 \quad 109 \quad 254 \quad 5 e-20 \quad 97.4$ ORF_isotig12422

gi|504970487|ref|WP_015157589.1| $40.49 \quad \begin{array}{llllll}163 & 82 & 6 & 13 & 474 & 80\end{array}$

236 6e-20 96.7 ORF_isotig12422 gi|505018197|ref|WP_015205299.1|

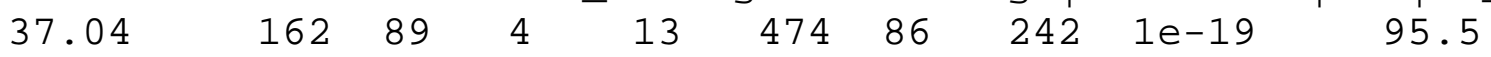

ORF_isotig12422 gi|748165414|ref|WP_039738990.1| $37.11 \quad 159 \quad 89$

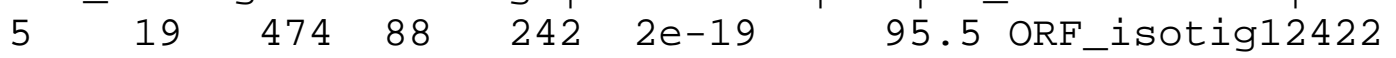

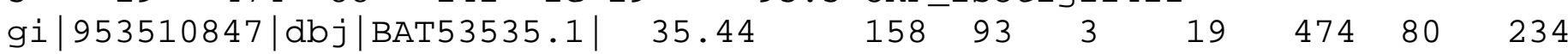

2e-19 95.5 ORF_isotig12422 gi|501377640|ref|WP_012409206.1| 
16198

$\begin{array}{llllll}3 & 16 & 474 & 82 & 239 & 2 \mathrm{e}-19\end{array}$

95.5

ORF_isotig12422

gi $|85544315| p d b|2 B T W| B \quad 38.18$

$\begin{array}{llll}165 & 90 & 6 & 19\end{array}$

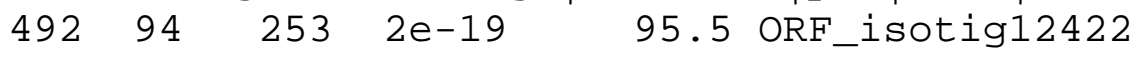

$\begin{array}{lllllllll}38.18 & 165 & 90 & 6 & 19 & 492 & 94 & 253 & 2 \mathrm{e}-19\end{array}$

gi $|85544314| p d b|2 B T W| A$

ORF_isotig12422 gi|737172391|ref|WP_035158595.1| $41.26 \quad 143 \quad 79$

$4 \quad 49 \quad 474 \quad 101 \quad 239 \quad 2 e-19 \quad 95.1 \quad$ ORF_isotig12422

gi|515888082|ref|WP_017318665.1| $35.19 \quad 162 \quad 92 \quad 5 \quad 16 \quad 474 \quad 85$

242 3e-19 94.7 ORF_isotig12422 gi|505020123|ref|WP_015207225.1|

$\begin{array}{llllllllll}35.86 & 145 & 90 & 2 & 37 & 471 & 100 & 241 & 3 e-19 & 94.7\end{array}$

ORF_isotig12422 gi|489705594|ref|WP_003609731.1| $39.24 \quad 15890$

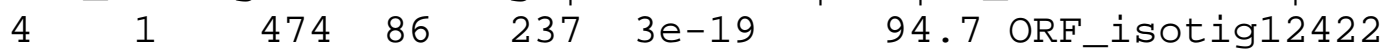

gi|504891436|ref|WP_015078538.1| $38.10 \quad 0 \quad 147 \quad 86 \quad 4 \quad 37 \quad 474 \quad 100$

242 4e-19 94.4 ORF_isotig12422 gi|763312982|ref|WP_044171432.1|

$\begin{array}{llllllllll}38.41 & 151 & 86 & 4 & 31 & 474 & 87 & 233 & 9 \mathrm{e}-19 & 93.2\end{array}$

ORF_isotig12422 gi|658419581|ref|WP_029646255.1| $37.24 \quad 145 \quad 87$

$3 \quad 34 \quad 468 \quad 100 \quad 240 \quad 1 \mathrm{e}-18 \quad 93.2$ ORF_isotig12422

gi|516956783|ref|WP_018183684.1| $38.82 \quad 0 \quad 152 \quad 87 \quad 4 \quad 28 \quad 474 \quad 87$

235 1e-18 93.2 ORF_isotig12422 gi|497231444|ref|WP_009545706.1| $\begin{array}{llllllllll}42.76 & 145 & 76 & 6 & 46 & 474 & 99 & 238 & 2 \mathrm{e}-18 & 92.8\end{array}$

$\begin{array}{llllll}\text { ORF_isotig12422 } & \text { gi|746961585|gb|KIF19114.1| } & 42.21 & 154 & 77 & 8\end{array}$

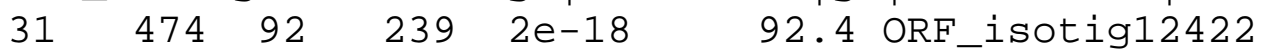

gi|917776332|ref|WP_052290273.1| $42.21 \quad 34 \quad 154 \quad 8 \quad 31 \quad 474 \quad 83$ 230 2e-18 92.4 ORF_isotig12422 gi|500232799|ref|WP_011902392.1|

$\begin{array}{llllllllll}37.41 & 147 & 83 & 4 & 31 & 462 & 87 & 227 & 3 \mathrm{e}-18 & 91.7\end{array}$

ORF_isotig12422 gi|515356232|ref|WP_016865554.1| $35.29 \quad 153 \quad 93$

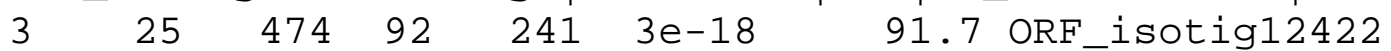

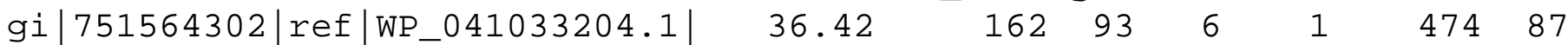

242 4e-18 91.7 ORF_isotig12422 gi|656037395|ref|WP_029076335.1|

$\begin{array}{llllllllll}35.26 & 156 & 96 & 3 & 25 & 492 & 89 & 239 & 5 e-18 & 90.9\end{array}$

ORF_isotig12422 gi|522080484|ref|WP_020591693.1| 38.46 $143 \quad 84$

$3 \quad 46 \quad 474 \quad 95 \quad 233 \quad 5 \mathrm{e}-18 \quad 90.9$ ORF_isotig12422

gi|495550750|ref|WP_008275329.1| $42.57 \quad \begin{array}{llllll}148 & 78 & 6 & 46 & 483 & 99\end{array}$

$2416 \mathrm{e}-18$ 90.9 ORF_isotig12422 gi|496167609|ref|WP_008892116.1|

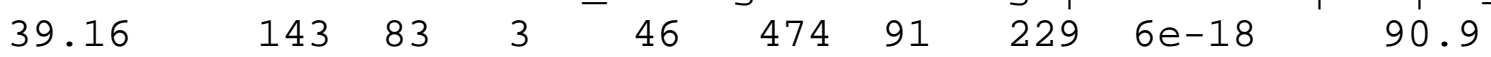

ORF_isotig12422 $\quad$ gi|427346779|gb|AFY29492.1| $37.84 \quad 148 \quad 87 \quad 4$

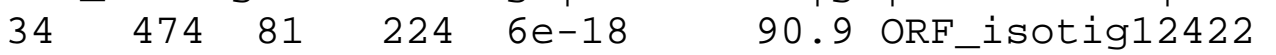

gi|504925739|ref|WP_015112841.1| $37.66 \quad 154 \quad 88 \quad 5 \quad 25 \quad 474 \quad 93$

$2427 \mathrm{e}-18 \quad 90.9$ ORF_isotig12422 gi|817130062|ref|WP_046502581.1|

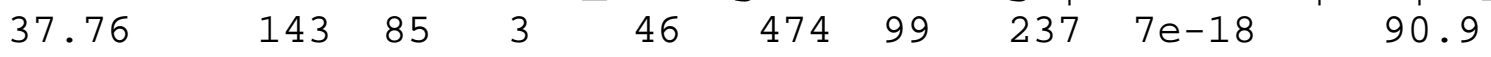

$\begin{array}{llllll}\text { ORF_isotig12422 } & \text { gi|1000089089|gb|KXJ56520.1| } & 39.16 & 143 & 83 & 3\end{array}$

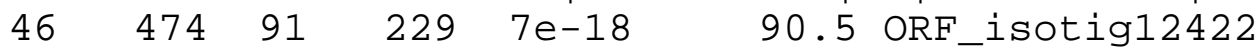

gi|522054471|ref|WP_020565680.1| $39.04 \quad 146 \quad 85 \quad 3 \quad 37 \quad 474 \quad 108$

249 7e-18 90.9 ORF_isotig12422 gi|504967338|ref|WP_015154440.1|

$\begin{array}{llllllllll}38.16 & 152 & 86 & 5 & 31 & 474 & 94 & 241 & 1 \mathrm{e}-17 & 90.5\end{array}$

ORF_isotig12422 gi|917319449|ref|WP_051926161.1| $38.19 \quad 144 \quad 84$

$4 \quad 46 \quad 474 \quad 103 \quad 242 \quad 1 \mathrm{e}-17 \quad 90.5$ ORF_isotig 12422

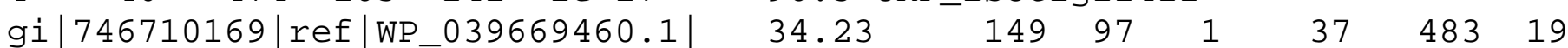

166 1e-17 88.6 ORF_isotig12422 gi|759607566|ref|WP_043325766.1|

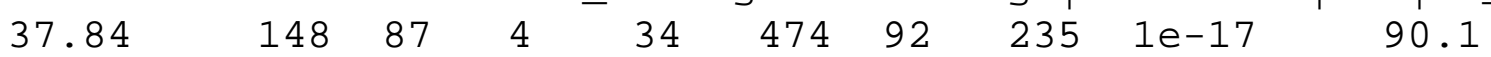

ORF_isotig12422 gi|501565810|ref|WP_012570258.1| $34.23 \quad 149 \quad 97$

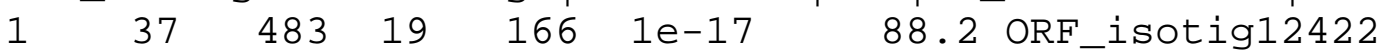

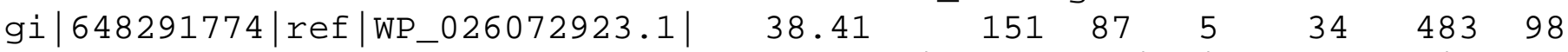

$2432 \mathrm{e}-17 \quad 89.7$ ORF_isotig12422 gi|827519218|gb|KLN61905.1|

$\begin{array}{llllllllll}37.06 & 143 & 86 & 3 & 46 & 474 & 83 & 221 & 2 \mathrm{e}-17 & 89.4\end{array}$

ORF_isotig12422 gi|920578875|ref|WP_053005650.1| $37.06 \quad 143 \quad 86$

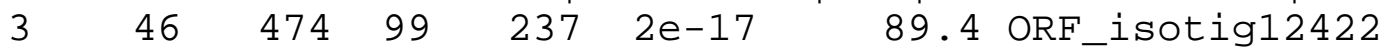

gi|740234212|ref|WP_038075400.1| $37.16 \quad-148 \quad 88 \quad 4 \quad 37 \quad 474 \quad 92$ 
$236 \quad 2 \mathrm{e}-17$

89.4 ORF_isotig12422

gi |652338179|ref |WP_026735392.1|

$\begin{array}{llllllllll}34.64 & 153 & 94 & 3 & 25 & 474 & 93 & 242 & 2 \mathrm{e}-17 & 89.4\end{array}$

ORF_isotig12422

gi|515385832|ref|WP_016877723.1| 35.95

15392

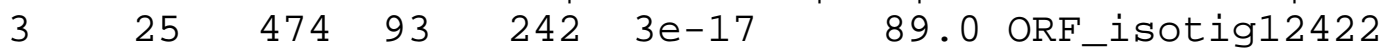

gi|915474789|ref|WP_050814322.1| $36.62 \quad 142 \quad 87 \quad 3 \quad 10 \quad 432 \quad 9$

148 3e-17 87.4 ORF_isotig12422 gi|916294818|ref|WP_051029864.1|

$\begin{array}{llllllllll}34.38 & 160 & 96 & 3 & 13 & 474 & 84 & 240 & 3 e-17 & 89.0\end{array}$

$\begin{array}{llllll}\text { ORF_isotig12422 } & \text { gi|91691483|gb|ABE34681.1| } & 37.84 & 148 & 88 & 3\end{array}$

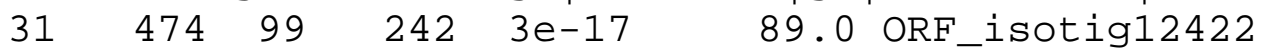

gi|156355949|ref|XP_001623696.1| $50.00 \quad 082 \quad 38 \quad 1 \quad 256 \quad 501 \quad 155$

233 3e-17 89.0 ORF_isotig12422 gi|872579356|ref|WP_048533013.1|

$\begin{array}{llllllllll}37.67 & 146 & 87 & 3 & 37 & 474 & 188 & 329 & 4 \mathrm{e}-17 & 89.7\end{array}$

ORF_isotig12422 $\quad$ gi|648499655|ref|WP_026191406.1| $36.14 \quad 166 \quad 97$

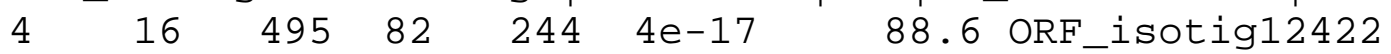

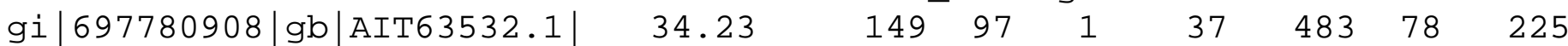

4e-17 88.2 ORF_isotig12422 gi|207082025|gb|ABS78257.2| 34.23

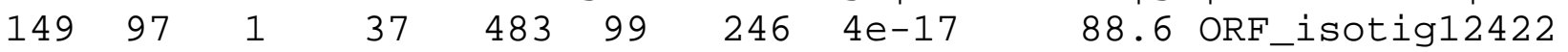

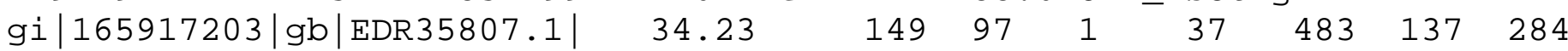

4e-17 89.0 ORF_isotig12422 gi|737933697|ref|WP_035898371.1|

$\begin{array}{llllllllll}38.22 & 157 & 91 & 5 & 10 & 474 & 84 & 236 & 5 e-17 & 88.2\end{array}$

ORF_isotig12422 gi|918692141|ref|WP_052561880.1| $35.46 \quad 141 \quad 87$

$3 \quad 52 \quad 474 \quad 88 \quad 224 \quad 6 \mathrm{e}-17 \quad 87.8$ ORF_isotig12422

gi|738342341|ref|WP_036294947.1| $36.14 \quad-166 \quad 97 \quad 4 \quad 16 \quad 495 \quad 87$

$2496 e-17$ 88.2 ORF_isotig12422 gi|941312503|ref|WP_055116231.1|

$\begin{array}{llllllllll}38.85 & 157 & 90 & 5 & 10 & 474 & 84 & 236 & 6 \mathrm{e}-17 & 87.8\end{array}$

ORF_isotig12422 gi|749301086|ref|WP_040125311.1| $37.84 \quad 148 \quad 88$

$3 \quad 31 \quad 474 \quad 149 \quad 292 \quad 8 e-17 \quad 88.2$ ORF_isotig12422

gi|916990632|ref|WP_051597344.1| $37.21 \quad 3129 \quad 79 \quad 2 \quad 34 \quad 420 \quad 99$

225 1e-16 87.4 ORF_isotig12422 gi|692118754|ref|WP_032075052.1|

$\begin{array}{llllllllll}33.56 & 149 & 98 & 1 & 37 & 483 & 19 & 166 & 1 \mathrm{e}-16 & 85.9\end{array}$

ORF_isotig12422 gi|797211671|ref|WP_045872614.1| $35.00 \quad 160 \quad 91$

$\begin{array}{llllllllll}5 & 19 & 474 & 88 & 242 & 1 \mathrm{e}-16 & 87.4 & \text { ORF_isotig12422 } & & \\ \text { gi|} 494317175|\mathrm{ref}| \mathrm{WP} & 007177410.1 \mid & 37.25 & 153 & 92 & 3 & 31 & 489 & 145\end{array}$

293 1e-16 87.8 ORF_isotig12422 gi|772943476|gb|AJX80078.1|

$\begin{array}{llllllllll}34.93 & 146 & 69 & 3 & 37 & 474 & 84 & 203 & 1 \mathrm{e}-16 & 86.7\end{array}$

ORF_isotig12422 gi|497652379|ref|WP_009966563.1| $34.93 \quad 146 \quad 69$

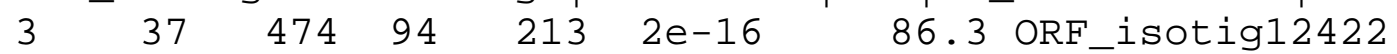

gi|493165631|ref|WP_006170876.1| $35.34 \quad-133 \quad 85 \quad 1 \quad 34 \quad 432 \quad 125$

256 2e-16 87.0 ORF_isotig12422 gi|656719681|gb|KEF42411.1|

$\begin{array}{llllllllll}37.41 & 147 & 87 & 4 & 37 & 474 & 79 & 221 & 2 \mathrm{e}-16 & 86.7\end{array}$

ORF_isotig12422 gi|504939174|ref|WP_015126276.1| $36.55 \quad 145 \quad 88$

$3 \quad 40 \quad 474 \quad 108 \quad 248 \quad 4 \mathrm{e}-16 \quad 85.9$ ORF_isotig12422

gi|501395618|ref|WP_012427184.1| $37.84 \quad 148 \quad 88 \quad 3 \quad 31 \quad 474 \quad 136$

279 6e-16 85.5 ORF_isotig12422 gi|651604058|ref|WP_026598946.1|

$\begin{array}{llllllllll}36.08 & 158 & 92 & 4 & 16 & 471 & 77 & 231 & 7 \mathrm{e}-16 & 84.7\end{array}$

ORF_isotig12422 gi|755598209|ref|WP_042525907.1| $32.89 \quad 149 \quad 99$

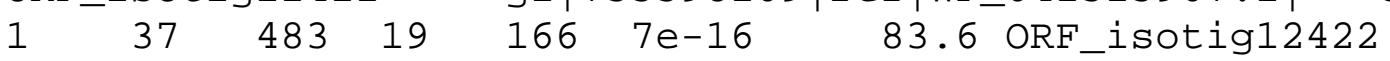

gi|640368848|ref|WP_024880427.1| $36.08 \quad-158 \quad 92 \quad 4 \quad 16 \quad 471 \quad 82$

236 1e-15 84.3 ORF_isotig12422 gi|1001837663|gb|KXS32259.1|

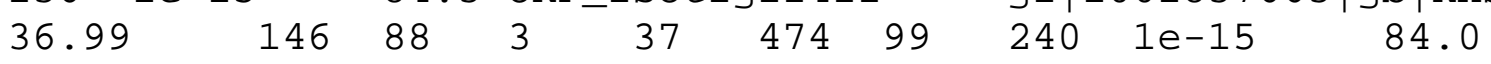

$\begin{array}{llllll}\text { ORF_isotig12422 } & \text { gi|931478713|gb|KPK59845.1| } & 37.80 & 127 & 77 & 2\end{array}$

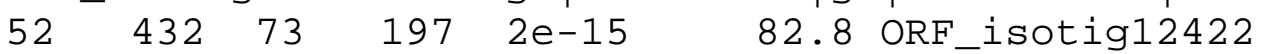

gi|926791675|ref|XP_013904311.1| $35.83 \quad 120 \quad 74 \quad 2 \quad 142 \quad 501 \quad 19$

135 2e-15 81.6 ORF_isotig12422 gi|156394135|ref|XP_001636682.1|

$\begin{array}{llllllllll}48.28 & 87 & 42 & 2 & 241 & 501 & 157 & 240 & 3 e-15 & 83.2\end{array}$

ORF_isotig12422 gi|497514158|ref|WP_009828356.1| $36.30 \quad 146 \quad 89$

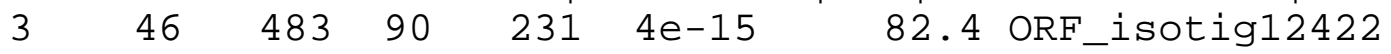


gi|754969497|ref|WP_042325399.1| 36.71

$15895 \quad 4$

$1474 \quad 119$

271 7e-15 82.4 ORF_isotig12422

gi |156379434|ref|XP_001631462.1|

$\begin{array}{llllllllll}45.83 & 72 & 39 & 0 & 241 & 456 & 147 & 218 & 7 \mathrm{e}-15 & 81.6\end{array}$

ORF_isotig12422 gi|752594371|ref|WP_041263758.1| $34.69 \quad 98 \quad 61$

$2 \quad 181 \quad 474 \quad 6 \quad 100 \quad 9 \mathrm{e}-15 \quad 78.6$ ORF_isotig12422

gi|738534441|ref|WP_036482850.1| $31.79 \quad 151 \quad 96 \quad 4 \quad 28 \quad 474 \quad 103$ 248 1e-14 81.3 ORF_isotig12422 gi|499442921|ref|WP_011130385.1|

$\begin{array}{llllllllll}36.57 & 134 & 82 & 3 & 34 & 432 & 105 & 236 & 2 \mathrm{e}-14 & 80.5\end{array}$

ORF_isotig12422 gi|739030777|ref|WP_036911908.1| $34.81 \quad 135 \quad 83$

$4 \quad 34 \quad 432 \quad 105 \quad 236 \quad 4 \mathrm{e}-14 \quad 79.7$ ORF_isotig12422

gi|499786267|ref|WP_011467001.1| $42.57 \quad-101 \quad 53 \quad 2 \quad 196 \quad 498 \quad 222$

317 5e-14 80.5 ORF_isotig12422 gi|123963072|gb|ABM77828.1|

$\begin{array}{llllllllll}33.78 & 148 & 93 & 4 & 34 & 474 & 105 & 248 & 6 \mathrm{e}-14 & 79.3\end{array}$

ORF_isotig12422 $\quad$ gi|331218212|ref|XP_003321784.1| $41.98 \quad 81 \quad 47$

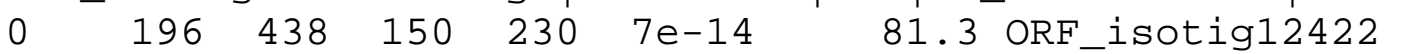

gi|550281002|ref|WP_022607401.1| $35.62 \quad-146 \quad 84 \quad 5 \quad 49 \quad 483 \quad 99$

$2359 \mathrm{e}-14$ 78.6 ORF_isotig12422 gi|800135199|dbj|GA034503.1|

$\begin{array}{llllllllll}36.55 & 145 & 88 & 3 & 40 & 474 & 90 & 230 & 2 \mathrm{e}-13 & 77.4\end{array}$

ORF_isotig12422 gi|331218210|ref|XP_003321783.1| $42.31 \quad 78 \quad 45$

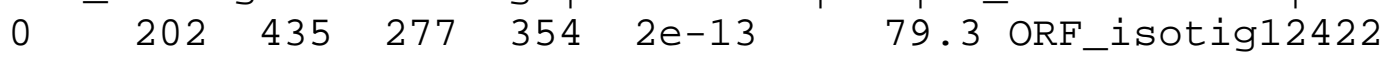

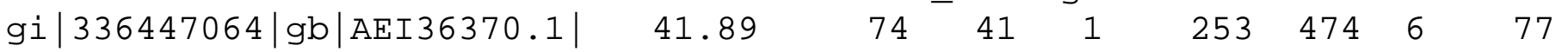

3e-13 73.9 ORF_isotig12422 gi|909606181|gb|KNE96553.1| 38.20

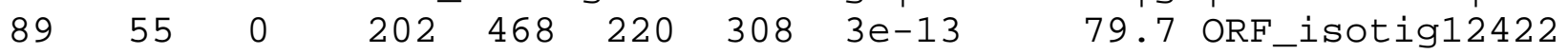

gi|831778908|ref|XP_012755554.1| $37.84 \quad 111 \quad 64 \quad 3 \quad 181 \quad 513 \quad 166$

$2713 e-13 \quad 79.3$ ORF_isotig12422 gi|290993528|ref|XP_002679385.1|

$\begin{array}{llllllllll}38.82 & 85 & 52 & 0 & 202 & 456 & 145 & 229 & 4 \mathrm{e}-13 & 76.6\end{array}$

ORF_isotig12422 gi|675852512|ref|XP_009011816.1| $38.74 \quad 111 \quad 66$

$1 \quad 211 \quad 543 \quad 206 \quad 314 \quad 6 \mathrm{e}-13 \quad 78.6 \quad$ ORF_isotig12422

gi|676386555|ref|XP_009036556.1| $41.76 \quad-91 \quad 52 \quad 1 \quad 205 \quad 474 \quad 152$ $2427 e-13 \quad 76.3$ ORF_isotig12422 gi|504984674|ref|WP_015171776.1|

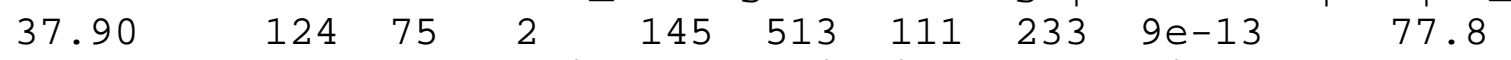

$\begin{array}{llllll}\text { ORF_isotig12422 } & \text { gi|568027492|gb|ETM01446.1| } & 37.00 & 100 & 60 & 2\end{array}$

$175 \quad 47459 \quad 155 \quad 1 e-12 \quad 75.1$ ORF_isotig12422

gi|999977806|gb|KXJ16980.1| $41.77 \quad-\begin{array}{lllllll}79 & 44 & 1 & 238 & 474 & 430 & 506\end{array}$

1e-12 77.8 ORF_isotig12422 gi|999977806|gb|KXJ16980.1| 32.61

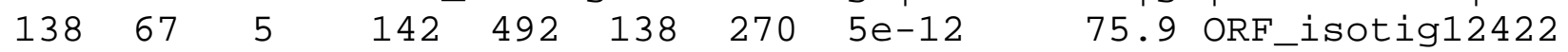

gi|909134564|gb|KNE60758.1| $33.61 \quad 119 \quad 76 \quad 2 \quad r \begin{array}{llll}145 & 501 & 24 & 139\end{array}$

1e-12 75.5 ORF_isotig12422 gi|586633751|gb|EWS64201.1| 39.18

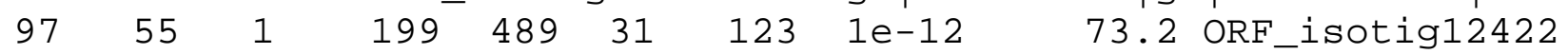

gi|999977804|gb|KXJ16978.1| $34.65 \quad 101 \quad 58 \quad 2 \quad 190 \quad 474 \quad 152 \quad 250$

1e-12 75.9 ORF_isotig12422 gi|737401579|ref|WP_035382755.1|

$\begin{array}{llllllllll}35.33 & 150 & 89 & 4 & 37 & 480 & 86 & 229 & 2 \mathrm{e}-12 & 75.1\end{array}$

ORF_isotig12422 gi|685951299|ref|XP_009269521.1| $38.54 \quad 96 \quad 57$

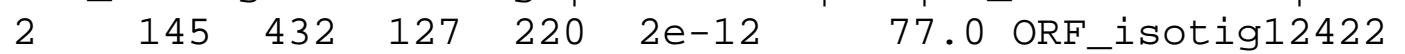

gi|524911978|ref|XP_@05110845.1| $35.87 \quad-92 \quad 59 \quad 0 \quad 145 \quad 420 \quad 116$

207 2e-12 77.0 ORF_isotig12422 gi|198417053|ref|XP_002128372.1|

$\begin{array}{llllllllll}33.07 & 127 & 84 & 1 & 145 & 525 & 110 & 235 & 2 \mathrm{e}-12 & 76.6\end{array}$

$\begin{array}{lllll}\text { ORF_isotig12422 } & \text { gi|219124481|ref|XP_002182531.1| } & 30.63 & 160 & 83\end{array}$

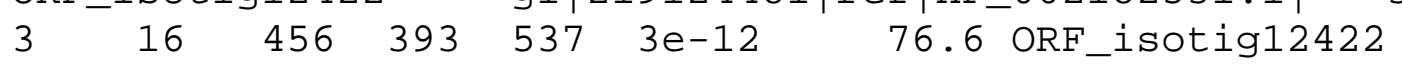

gi|909134565|gb|KNE60759.1| $33.61 \quad 119 \quad 76 \quad 2 \quad 145 \quad 501 \quad 93 \quad 208$

3e-12 75.1 ORF_isotig12422 gi|909134566|gb|KNE60760.1| 33.61

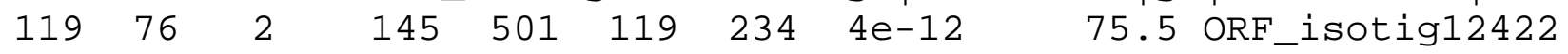

gi|727439853|ref|XP_010501307.1| $37.63 \quad 03 \quad 58 \quad 0 \quad \begin{array}{lllll}142 & 420 & 108\end{array}$

$2004 \mathrm{e}-12$ 76.6 ORF_isotig12422 gi|727439853|ref|XP_010501307.1|

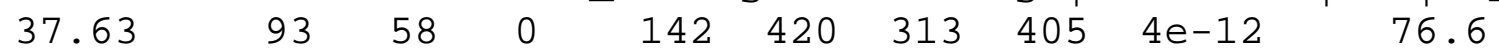

$\begin{array}{llllll}\text { ORF_isotig12422 } & \text { gi|909138881|gb|KNE64222.1| } & 33.61 & 119 & 76 & 2\end{array}$

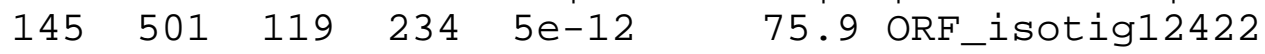




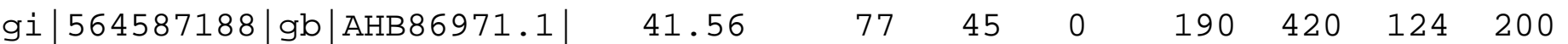

6e-12 75.9 ORF_isotig12422 gi|223995009|ref|XP_002287188.1|

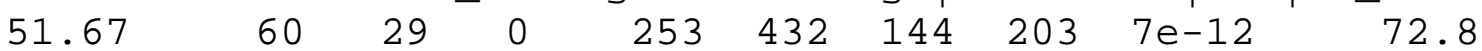

ORF_isotig12422 gi|505014688|ref|WP_015201790.1| $38.83 \quad 103 \quad 58$

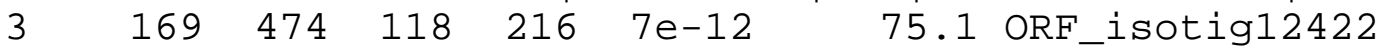

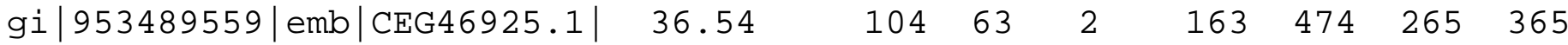

7e-12 75.1 ORF_isotig12422 gi|731346801|ref|XP_010684647.1|

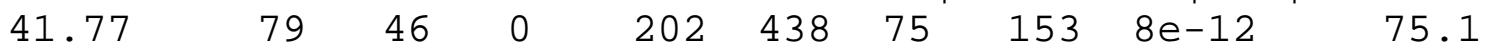

$\begin{array}{llllll}\text { ORF_isotig12422 } & \text { gi|983147566|gb|KWU45526.1| } & 39.39 & 99 & 57 & 2\end{array}$

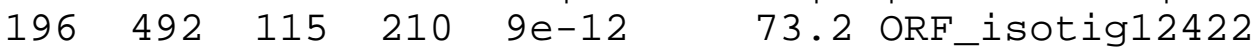

gi|731346799|ref|XP_010684646.1| $41.77 \quad 0 \quad 79 \quad 46 \quad 0 \quad 202 \quad 438 \quad 129$

207 9e-12 75.1 ORF_isotig12422 gi|490267384|ref|WP_004163951.1|

$\begin{array}{llllllllll}29.45 & 146 & 91 & 2 & 28 & 438 & 88 & 230 & 1 \mathrm{e}-11 & 72.8\end{array}$

ORF_isotig12422 gi|727603973|ref|XP_010475030.1| $37.78 \quad 90 \quad 56$

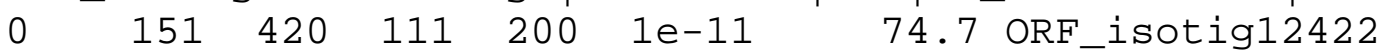

gi|752845383|ref|WP_041474394.1| $29.45 \quad 146 \quad 91 \quad 2 \quad 28 \quad 438 \quad 102$

244 1e-11 73.2 ORF_isotig12422 gi|727569811|ref|XP_010457419.1|

$\begin{array}{llllllllll}37.78 & 90 & 56 & 0 & 151 & 420 & 111 & 200 & 1 \mathrm{e}-11 & 74.7\end{array}$

ORF_isotig12422 $\quad$ gi|312171048|emb|CBX79307.1| $29.45 \quad 146 \quad 91 \quad 2$

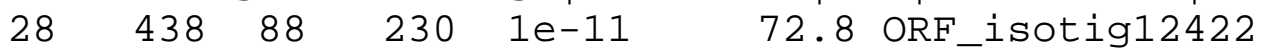

gi|470239215|ref|XP_004351373.1| $35.14 \quad 111 \quad 66 \quad 2 \quad 190 \quad 519 \quad 475$

580 1e-11 75.1 ORF_isotig12422 gi|490258075|ref|WP_004155296.1|

$\begin{array}{llllllllll}29.45 & 146 & 91 & 2 & 28 & 438 & 102 & 244 & 1 \mathrm{e}-11 & 72.8\end{array}$

ORF_isotig12422 $\quad$ gi|310766388|gb|ADP11338.1| $29.45 \quad 146 \quad 91 \quad 2$

$28 \quad 438 \quad 115 \quad 257 \quad 1 \mathrm{e}-11 \quad 73.2$ ORF_isotig12422

gi|219111005|ref|XP_002177254.1| $34.75 \quad 118 \quad 65 \quad 3 \quad 145 \quad 474 \quad 108$

221 1e-11 72.4 ORF_isotig12422 gi|478729310|emb|CCP05801.1|

$\begin{array}{llllllllll}29.45 & 146 & 91 & 2 & 28 & 438 & 102 & 244 & 1 \mathrm{e}-11 & 72.8\end{array}$

$\begin{array}{llllll}\text { ORF_isotig12422 } & \text { gi|635369185|emb|CCI42660.1| } & 34.38 & 128 & 80 & 4\end{array}$

$145 \quad 525 \quad 125249$ 1e-11 73.2 ORF_isotig12422

gi|752845037|ref|WP_041474048.1| $29.45 \quad 146 \quad 91 \quad 2 \quad 28 \quad 438 \quad 102$

244 1e-11 72.8 ORF_isotig12422 gi|817516937|ref|WP_046572938.1|

$\begin{array}{llllllllll}37.34 & 158 & 94 & 4 & 1 & 474 & 126 & 278 & 1 \mathrm{e}-11 & 73.2\end{array}$

ORF_isotig12422 gi|490272265|ref|WP_004168384.1| 29.45 14691

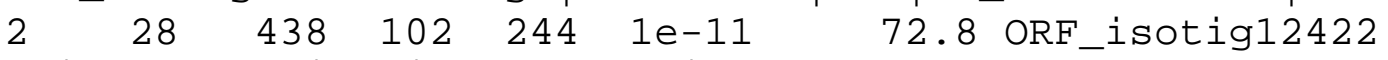

gi|224965368|emb|CAX56900.1| $29.45 \quad 146 \quad 91 \quad 2 \quad 28 \quad 438 \quad 115 \quad 257$

1e-11 72.8 ORF_isotig12422 gi|297848618|ref|XP_002892190.1|

$\begin{array}{llllllllll}37.78 & 90 & 56 & 0 & 151 & 420 & 111 & 200 & 2 e-11 & 74.3\end{array}$

$\begin{array}{llllll}\text { ORF_isotig12422 } & \text { gi|15219610|ref|NP_171894.1| } & 37.78 & 90 & 56 & \odot\end{array}$

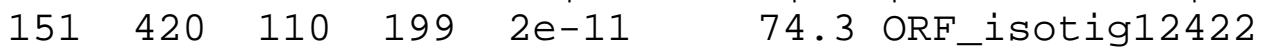

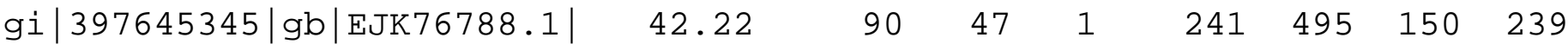

2e-11 74.3 ORF_isotig12422 gi|551588562|ref|XP_005778952.1|

$\begin{array}{llllllllll}38.38 & 99 & 58 & 2 & 178 & 474 & 103 & 198 & 2 \mathrm{e}-11 & 71.6\end{array}$

ORF_isotig12422 gi|301112447|ref|XP_002997994.1| $37.00 \quad 100 \quad 60$

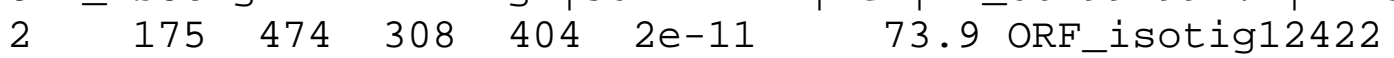

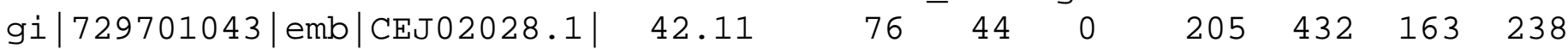

2e-11 73.9 ORF_isotig12422 gi|754352951|ref|XP_011270847.1|

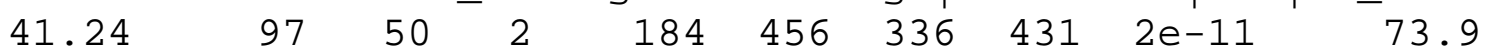

ORF_isotig12422 gi|562746587|ref|WP_023653709.1| 30.00 $\quad 140 \quad 86$

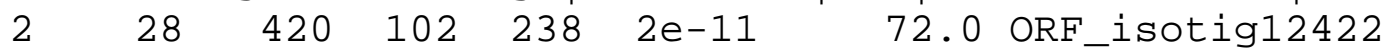

gi|922346013|ref|XP_013449921.1| $36.56 \quad-93 \quad 59 \quad 0 \quad 190 \quad 468 \quad 125$

217 2e-11 73.9 ORF_isotig12422 gi|729710313|emb|CEI93301.1|

$\begin{array}{llllllllll}42.11 & 76 & 44 & 0 & 205 & 432 & 66 & 141 & 2 \mathrm{e}-11 & 73.6\end{array}$

$\begin{array}{llllll}\text { ORF_isotig12422 } & \text { gi|215983522|gb|ACJ71777.1| } & 37.08 & 89 & 56 & 0\end{array}$

$202 \quad 468 \quad 128 \quad 216 \quad 2 e-11 \quad 71.6$ ORF_isotig12422

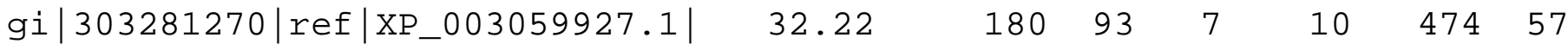

232 2e-11 71.6 ORF_isotig12422 gi|224001048|ref|XP_002290196.1| 
$\begin{array}{llllllllll}32.69 & 104 & 62 & 2 & 145 & 435 & 122 & 224 & 3 e-11 & 71.6\end{array}$

ORF_isotig12422

gi $|552916579| \mathrm{gb} \mid \mathrm{ESA01781.1|} \quad 42.67$

$75 \quad 43$

$\odot$

$208 \quad 432 \quad 124 \quad 198 \quad 3 e-11 \quad 71.2$ ORF_isotig12422

gi|727146284|emb|CEG71516.1| $42.11 \quad-76 \quad 44 \quad 0 \quad 0205432 \quad 139 \quad 214$

3e-11 73.6 ORF_isotig12422 gi|66803020|ref|XP_635353.1| 33.64

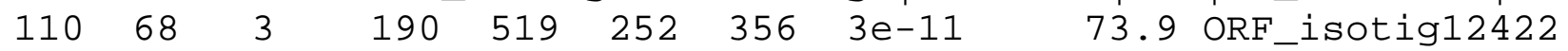

gi|727146285|emb|CEG71517.1| $42.11 \quad 46 \quad 44 \quad 0 \quad 0205432 \quad 163 \quad 238$

3e-11 73.6 ORF_isotig12422 gi|675213985|ref|XP_008915877.1|

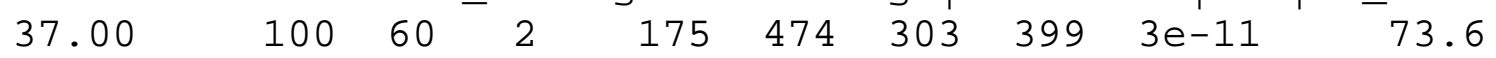

$\begin{array}{llllll}\text { ORF_isotig12422 } & \text { gi|570996289|gb|ETP52930.1| } & 37.00 & 100 & 60 & 2\end{array}$

$175 \quad 474 \quad 302 \quad 398 \quad 3 e-11 \quad 73.6$ ORF_isotig12422

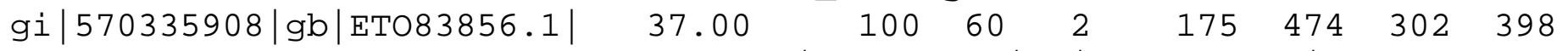
3e-11 73.6 ORF_isotig12422 gi|568057987|gb|ETM54635.1| 37.00

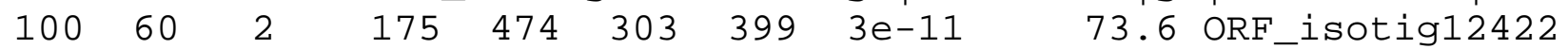

gi|444891677|gb|AGE13358.1| $51.67 \quad 60 \quad 29 \quad 0 \quad-253 \quad 432 \quad 230 \quad 289$

3e-11 72.8 ORF_isotig12422 gi|570959955|gb|ETP24934.1| 37.00

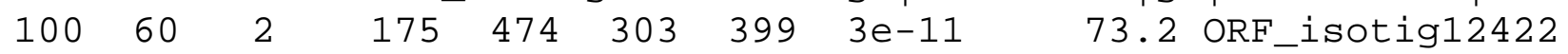

gi|330794008|ref|XP_003285073.1| $40.26 \quad 77 \quad 46 \quad 0 \quad \begin{array}{lllll}190 & 420 & 160\end{array}$

236 3e-11 73.6 ORF_isotig12422 gi|388497750|gb|AFK36941.1|

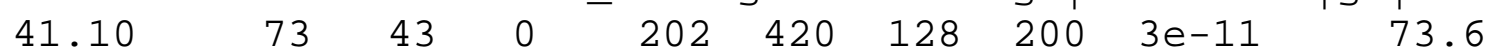

ORF_isotig12422 gi|122202937|sp|Q2QKL5.1|PCS3_LOTJA 41.10

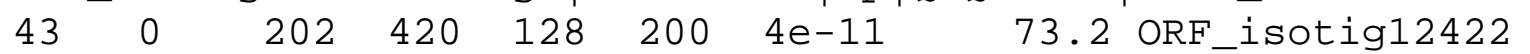

gi|147719992|sp|Q2TE74.2|PCS2_LOTJA $41.10 \quad 73 \quad 43 \quad 0 \quad 202 \quad 420$

$1282004 \mathrm{e}-11$ 73.2 ORF_isotig12422 gi|50659119|gb|AAT80341.1|

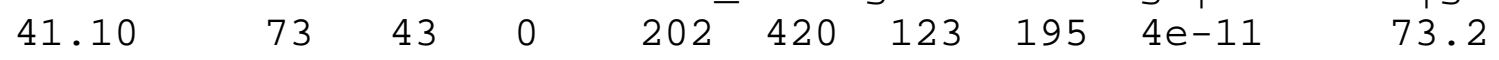

$\begin{array}{llllll}\text { ORF_isotig12422 } & \text { gi|568027478|gb|ETM01432.1| } & 35.29 & 102 & 63 & 2\end{array}$

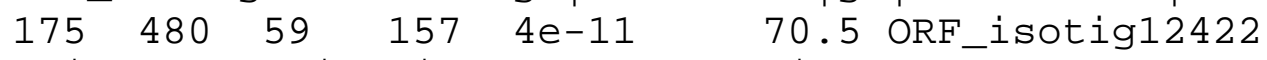

gi|593799054|ref|XP_007162565.1| $42.86 \quad 77 \quad 44 \quad 0 \quad 190 \quad 420 \quad 125$

$2014 \mathrm{e}-11$ 73.2 ORF_isotig12422 gi|902234844|gb|KNA23610.1|

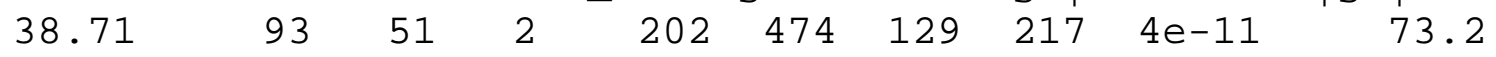

$\begin{array}{llllll}\text { ORF_isotig12422 } & \text { gi|281202856|gb|EFA77058.1| } & 37.66 & 77 & 48 & \odot\end{array}$

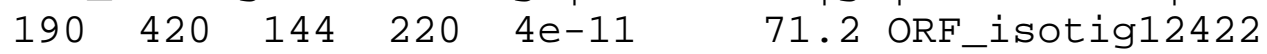

gi|758352771|dbj|GAN05107.1| $33.94 \quad 109 \quad 57 \quad 2 \quad 253 \quad 534 \quad 238 \quad 346$

4e-11 73.6 ORF_isotig12422 gi|570959958|gb|ETP24937.1| 35.29

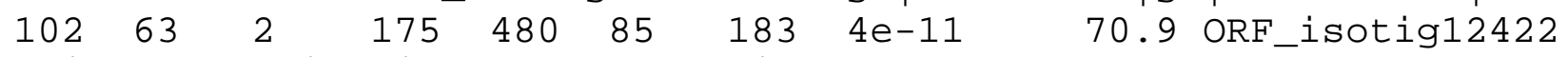

gi|518404331|ref|WP_019574538.1| $45.95 \quad 34 \quad 38 \quad 1 \quad \begin{array}{lllll}1 & 253 & 474 & 182\end{array}$

253 5e-11 71.2 ORF_isotig12422 gi|325516458|gb|ADZ24787.1|

$\begin{array}{llllllllll}36.67 & 90 & 57 & 0 & 151 & 420 & 111 & 200 & 5 \mathrm{e}-11 & 72.8\end{array}$

ORF_isotig12422 gi|590715531|ref|XP_007050222.1| $36.56 \quad 93 \quad 56$

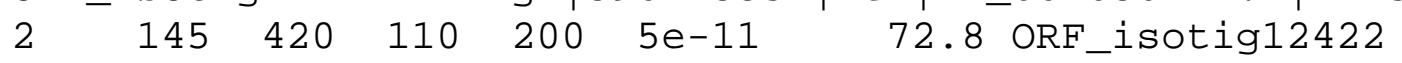

gi|970660098|gb|KUF97679.1| $37.00 \quad 100 \quad 60 \quad 2 \quad \begin{array}{llllll}175 & 474 & 303 & 399\end{array}$

6e-11 72.8 ORF_isotig12422 gi|654738300|ref|WP_028194554.1|

$\begin{array}{llllllllll}36.71 & 158 & 95 & 4 & 1 & 474 & 126 & 278 & 6 \mathrm{e}-11 & 71.2\end{array}$

ORF_isotig12422 gi|736937357|ref|WP_034934372.1| $32.41 \quad 108 \quad 71$

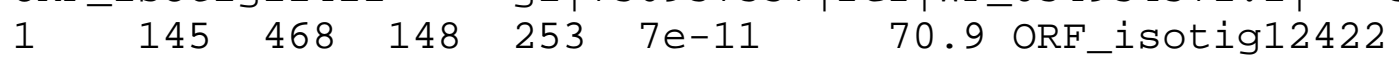

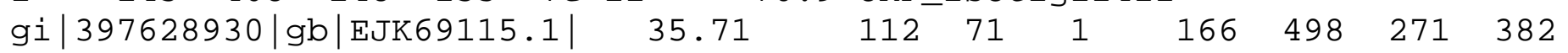

7e-11 72.4 ORF_isotig12422 gi|737586499|ref|WP_035557439.1|

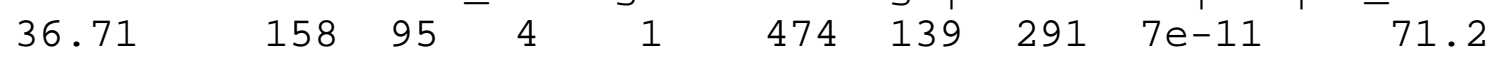

$\begin{array}{llrrrr}\text { ORF_isotig12422 } & \text { gi|357575758|gb|AET85547.1| } & 32.69 & 104 & 62 & 2\end{array}$

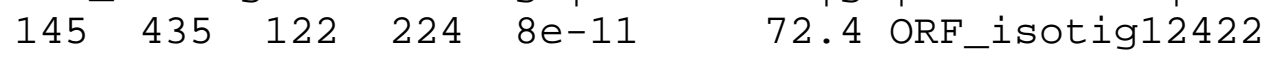

gi|599364119|ref|XP_007405638.1| $40.26 \quad 77 \quad 46 \quad 0 \quad 202 \quad 432 \quad 159$

235 8e-11 70.5 ORF_isotig12422 gi|568057990|gb|ETM54638.1|

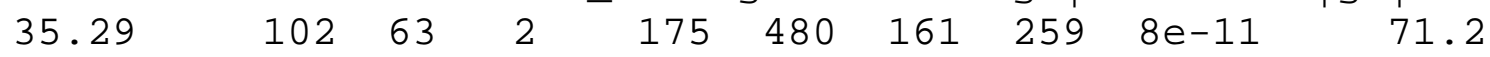

$\begin{array}{lllll}\text { ORF_isotig12422 gi|823127937|ref|XP_012440935.1| } 40.26 & 77 & 46\end{array}$

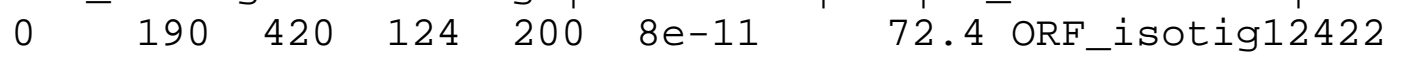

gi|823127935|ref|XP_012440927.1| $40.26 \quad-77 \quad 46 \quad 0 \quad 190 \quad 420 \quad 124$

200 8e-11 72.4 ORF_isotig12422 gi|907093431|gb|KNC99293.1| 
45.31

$64 \quad 35$

$\odot$

$241432 \quad 201 \quad 264 \quad 8 e-11$

72.4

ORF_isotig12422

$145 \quad 465 \quad 110 \quad 209$ gi $\mid 661892612$ | emb |CDP03770.1| 35.51

$107 \quad 62 \quad 2$

\subsection{0}

\begin{tabular}{ll|lccccc} 
gi|823127939|ref|XP_012440942.1| 40.26 & 77 & 46 & $\odot$ & 190 & 420 \\
138 & $8 e-11$ & 72.0 & ORF_isotig12422 & gi|566032216|gb|ETI55127.1|
\end{tabular}

$8 \mathrm{e}-11$

72.4 ORF_isotig12422

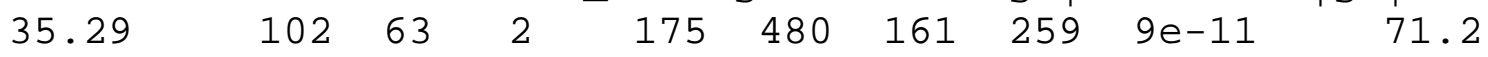

ORF_isotig12422 $\quad$ gi|4322421|gb|AAD16046.1| $39.73 \quad 73 \quad 44 \quad \odot$

$202 \quad 420 \quad 128 \quad 200 \quad 9 e-11 \quad 72.0 \quad$ ORF_isotig12422

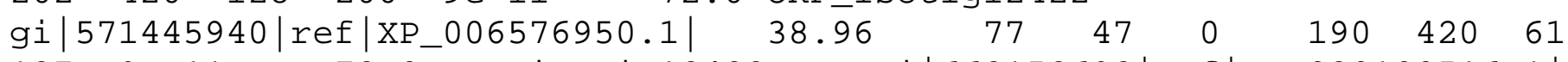

137 9e-11 72.0 ORF_isotig12422 gi|662152692|ref|WP_030100516.1|

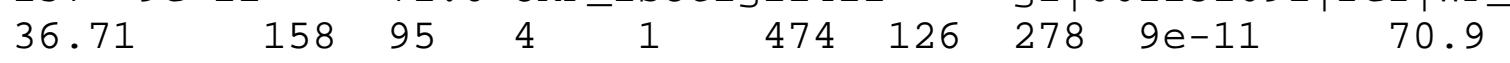

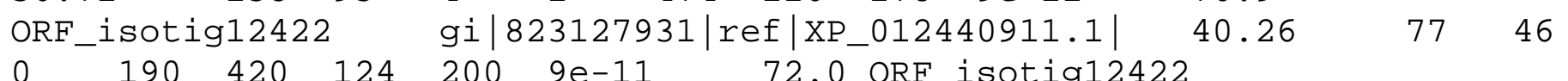

$\begin{array}{lllllllll}0 & 190 & 420 & 124 & 200 & 9 e-11 & 72.0 & \text { ORF_isotig12422 } & \\ \text { gi|908401434|ref|XP_O13070252.1| } & 38.46 & 104 & 58 & 2 & 202 & 513 & 130\end{array}$

$2279 e-11$ 72.0 ORF_isotig12422 gi|763743549|gb|KJB11048.1|

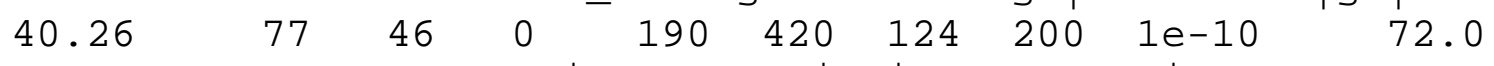

$\begin{array}{llllll}\text { ORF_isotig12422 } & \text { gi|734345461|gb|KHN10759.1| } & 38.96 & 77 & 47 & 0\end{array}$

$190 \quad 420 \quad 123 \quad 199 \quad 1 e-10 \quad 72.0$ ORF_isotig12422

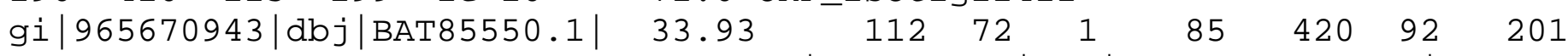

1e-10 72.0 ORF_isotig12422 gi|823127933|ref|XP_012440917.1|

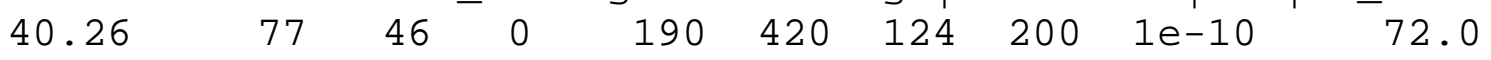

$\begin{array}{llllll}\text { ORF_isotig12422 } & \text { gi|325186679|emb|CCA21228.1| } & 37.04 & 108 & 65 & 3\end{array}$

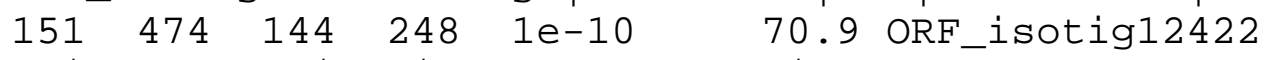

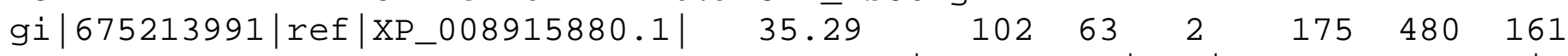
259 1e-10 70.9 ORF_isotig12422 gi|950949688|ref|XP_014495217.1|

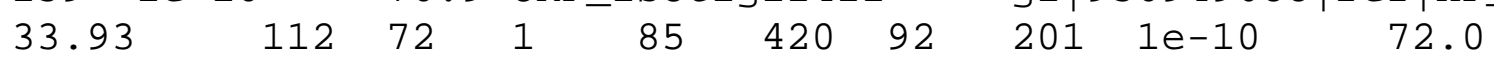

$\begin{array}{llllll}\text { ORF_isotig12422 } & \text { gi|570335911|gb|ET083859.1| } & 35.29 & 102 & 63 & 2\end{array}$

$175 \quad 480 \quad 161 \quad 259 \quad 1 e-10 \quad 70.9$ ORF_isotig12422

gi|763743550|gb|KJB11049.1| $40.26 \quad-77 \quad 46 \quad 0 \quad 190 \quad 420 \quad 124 \quad 200$

1e-10 72.0 ORF_isotig12422 gi|570996278|gb|ETP52920.1| 35.29

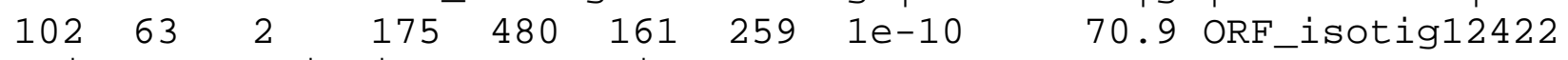

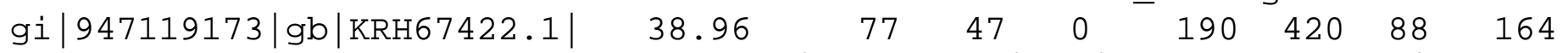

1e-10 71.6 ORF_isotig12422 gi|356505041|ref|XP_003521301.1|

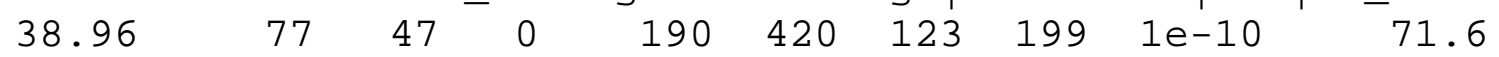

ORF_isotig12422 $\quad$ gi|167537519|ref|XP_-001750428.1| $31.69 \quad 142 \quad 95$

$2 \quad 37456 \quad 105 \quad 246 \quad 1 \mathrm{e}-10 \quad 70.1$ ORF_isotig12422

gi|727647674|ref|XP_010494405.1| $37.08 \quad-89 \quad 56 \quad 0 \quad 0202468 \quad 128$

216 1e-10 71.6 ORF_isotig12422 gi|675852508|ref|XP_009011814.1|

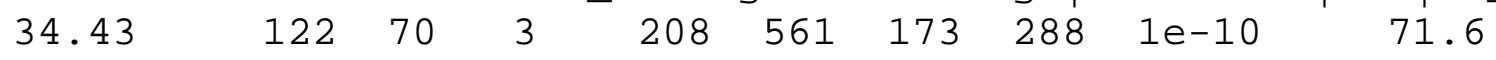

$\begin{array}{llllll}\text { ORF_isotig12422 } & \text { gi|674249977|gb|KFK42742.1| } & 35.56 & 90 & 58 & 0\end{array}$

$151 \quad 420 \quad 111200 \quad 1 \mathrm{e}-10 \quad 71.6$ ORF_isotig12422

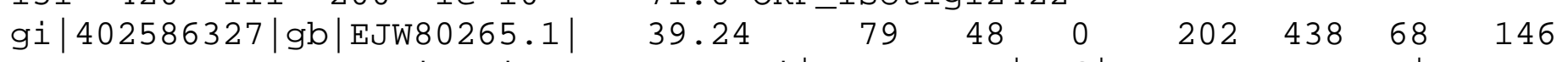

1e-10 69.3 ORF_isotig12422 gi|567185115|ref|XP_006403146.1|

$\begin{array}{llllllllll}38.20 & 89 & 55 & 0 & 202 & 468 & 128 & 216 & 1 \mathrm{e}-10 & 71.6\end{array}$

ORF_isotig12422 gi|449443293|ref|XP_004139414.1| $35.71 \quad 98 \quad 59$

$2 \quad 202495 \quad 53 \quad 146 \quad 1 \mathrm{e}-10 \quad 68.2$ ORF_isotig12422

gi|551561391|ref|XP_005767549.1| $40.00 \quad 060000000$

239 1e-10 69.7 ORF_isotig12422 gi|290980155|ref|XP_002672798.1|

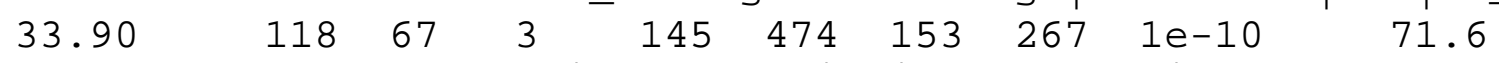

ORF_isotig12422 $\quad$ gi|284466089|gb|ACL00594.3| $39.73 \quad 73 \quad 44 \quad 0$

$202 \quad 420 \quad 128 \quad 200 \quad 2 e-10 \quad 71.2$ ORF_isotig12422

gi|549049394|emb|CCX11465.1| $35.51 \quad \begin{array}{lllllll}107 & 65 & 3 & 145 & 462 & 127 & 230\end{array}$ 2e-10 71.2 ORF_isotig12422 gi|21104518|dbj|BAB93120.1| 34.26

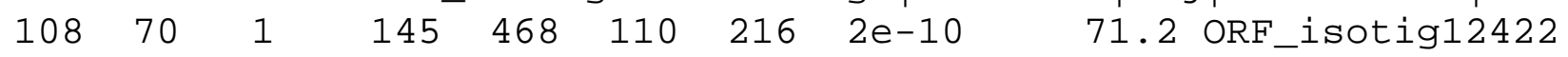

gi|694541843|ref|XP_009497159.1| $37.50 \quad 06 \quad 59 \begin{array}{lllll}1 & 145 & 432 & 48\end{array}$

142 2e-10 71.2 ORF_isotig12422 gi|493073505|ref|WP_006122095.1|

$\begin{array}{llllllllll}31.52 & 92 & 63 & 0 & 145 & 420 & 148 & 239 & 2 \mathrm{e}-10 & 69.7\end{array}$ 
ORF_isotig12422

gi $|814540591|$ emb |CEQ42050.1| 37.80

82

510

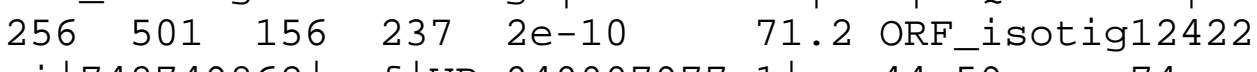

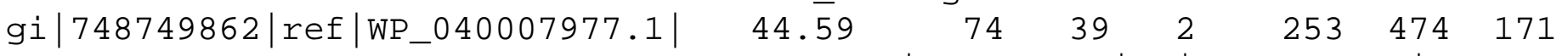

242 2e-10 69.7 ORF_isotig12422 gi|870854053|gb|KMT05872.1|

$\begin{array}{llllllllll}41.10 & 73 & 43 & 0 & 202 & 420 & 104 & 176 & 2 \mathrm{e}-10 & 71.2\end{array}$

$\begin{array}{llllll}\text { ORF_isotig12422 } & \text { gi|870854052|gb|KMT05871.1| } & 41.10 & 73 & 43 & 0\end{array}$

$202420 \quad 129201 \quad 2 \mathrm{e}-10 \quad 71.2$ ORF_isotig12422

gi|870854054|gb|KMT05873.1| $41.10 \quad-73 \quad 43 \quad 0 \quad 0202420 \quad 145 \quad 217$

2e-10 71.2 ORF_isotig12422 gi|970651949|gb|KUF90697.1| 35.29

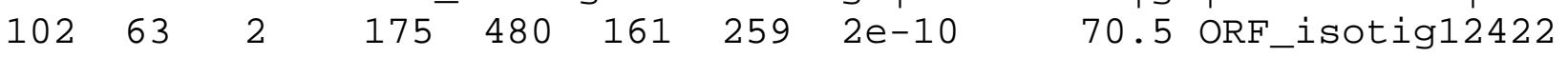

gi|902234842|gb|KNA23608.1| $41.10 \quad 73 \quad 43 \quad 0 \quad \begin{array}{lllll}202 & 420 & 129 & 201\end{array}$

2e-10 71.2 ORF_isotig12422 gi|588263371|ref|XP_006960829.1|

$\begin{array}{llllllllll}36.36 & 77 & 49 & 0 & 202 & 432 & 160 & 236 & 2 \mathrm{e}-10 & 70.1\end{array}$

ORF_isotig12422 $\quad$ gi|15240084|ref|NP_199220.1| $39.73 \quad 73 \quad 44 \quad 0$

$202 \quad 420 \quad 128 \quad 200 \quad 2 \mathrm{e}-10 \quad 71.2$ ORF_isotig12422

gi|18254401|gb|AAL66747.1|AF461180_1 $39.73 \quad 33 \quad 44 \quad 0 \quad 202420$

$128200 \quad 2 \mathrm{e}-10 \quad 71.2$ ORF_isotig12422

gi|694541835|ref|XP_009497157.1| $37.50 \quad 0 \quad 96 \quad 59 \quad 1 \quad 145 \quad 432 \quad 315$

$409 \quad 2 \mathrm{e}-10 \quad 71.6$ ORF_isotig12422

gi|7229390|gb|AAF42805.1|AF162689_1 $39.73 \quad 73 \quad 44 \quad 0 \quad 202420$

$1282002 \mathrm{e}-10 \quad 71.2$ ORF_isotig12422 gi|870854055|gb|KMT05874.1|

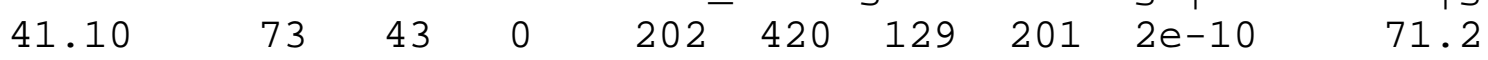

ORF_isotig12422 gi|4768281|gb|AAD29446.1|AF085231_2 $37.08 \quad 89$

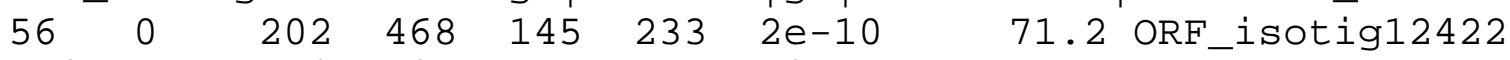

gi|729349445|ref|XP_010542900.1| $35.96 \quad 089 \quad 57 \quad 0 \quad 0202468 \quad 128$

216 2e-10 71.2 ORF_isotig12422 gi|384491545|gb|EIE82741.1|

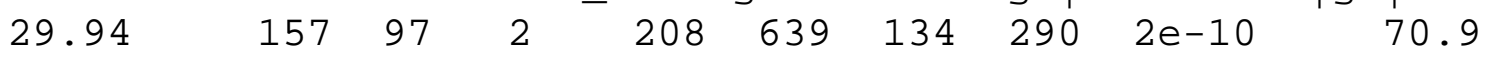

ORF_isotig12422 $\quad$ gi|671683790|emb|CDS12824.1| $41.56 \quad 77 \quad 45 \quad \odot$

$202 \quad 432 \quad 206 \quad 282 \quad 2 \mathrm{e}-10 \quad 71.2$ ORF_isotig12422

gi|731346805|ref|XP_010684649.1| $41.10 \quad 73 \quad 43 \quad 0 \quad 0202420 \quad 181$

$2532 \mathrm{e}-10 \quad 71.2$ ORF_isotig12422 gi|731346807|ref|XP_010684650.1|

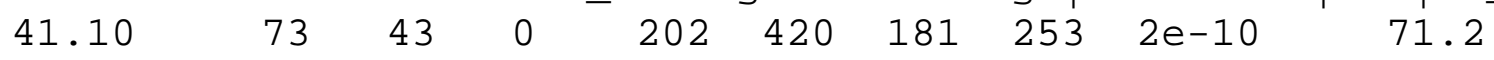

$\begin{array}{llllll}\text { ORF_isotig12422 } & \text { gi|13928024|emb|CAC37692.1| } & 36.26 & 91 & 56 & 1\end{array}$

$202 \quad 474 \quad 128 \quad 216 \quad 2 \mathrm{e}-10 \quad 70.9$ ORF_isotig12422

gi|470508086|ref|XP_004367696.1| $40.00 \quad 075 \quad 45 \quad 0 \quad 196 \quad 420 \quad 108$

182 2e-10 70.9 ORF_isotig12422 gi|902234843|gb|KNA23609.1|

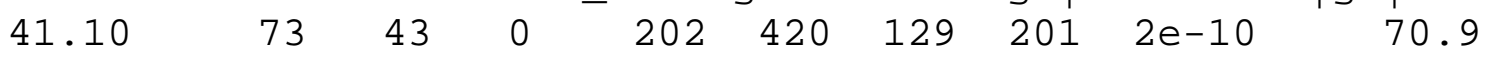

ORF_isotig12422 gi|685326730|ref|XP_009101739.1| $36.26 \quad 91 \quad 56$

$1 \quad 202 \quad 474 \quad 128 \quad 216 \quad 2 \mathrm{e}-10 \quad 70.9$ ORF_isotig12422

gi|443301362|gb|AGC82138.1| $39.73 \quad 33 \quad 44 \quad 0 \quad 0 \begin{array}{llllll}202 & 420 & 129 & 201\end{array}$

2e-10 70.9 ORF_isotig12422 gi|520899875|ref|WP_020322885.1|

$\begin{array}{llllllllll}31.31 & 99 & 68 & 0 & 145 & 441 & 148 & 246 & 2 \mathrm{e}-10 & 69.3\end{array}$

ORF_isotig12422 gi|731346810|ref|XP_010684651.1| $41.10 \quad 73 \quad 43$

$\odot \quad 202 \quad 420 \quad 120 \quad 192 \quad 2 \mathrm{e}-10 \quad 70.9$ ORF_isotig12422

gi|731346803|ref|XP_010684648.1| $41.10 \quad-73 \quad 43 \quad 0 \quad 202 \quad 420 \quad 181$

253 2e-10 70.9 ORF_isotig12422 gi|301128435|gb|ADK61091.1|

$\begin{array}{llllllllll}41.10 & 73 & 43 & \odot & 202 & 420 & 128 & 200 & 2 \mathrm{e}-10 & 70.9\end{array}$

$\begin{array}{lllll}\text { ORF_isotig12422 gi|297794999|ref|XP_002865384.1| } & 39.73 & 73 & 44\end{array}$

$\odot \quad 202 \quad 420 \quad 128 \quad 200 \quad 3 e-10 \quad 70.9$ ORF_isotig12422

gi|923860545|ref|XP_013706438.1| $36.26 \quad-91 \quad 56 \quad 1 \quad 202 \quad 474 \quad 185$

273 3e-10 70.9 ORF_isotig12422 gi|565432669|ref|XP_006280379.1|

$\begin{array}{llllllllll}34.86 & 109 & 68 & 2 & 145 & 468 & 110 & 216 & 3 e-10 & 70.5\end{array}$

$\begin{array}{lllll}\text { ORF_isotig12422 } & \text { gi|170594708|ref|XP_001902100.1| } & 39.24 & 79 & 48\end{array}$

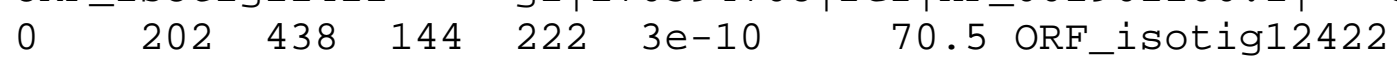

gi|702451810|ref|XP_010025777.1| $39.19 \quad-74 \quad 45 \quad 0 \quad 199 \quad 420 \quad 144$

217 3e-10 68.9 ORF_isotig12422 gi|284468345|gb|ABW98498.2 |

$\begin{array}{llllllllll}39.73 & 73 & 44 & \odot & 202 & 420 & 128 & 20 \odot & 3 e-10 & 70.5\end{array}$ 
ORF_isotig12422

gi $|511010013| \mathrm{gb}|\mathrm{EPB} 91244.1| \quad 45.00$

60

$33 \odot$

$253 \quad 432 \quad 233292 \quad 3 e-10 \quad 70.9$ ORF_isotig12422

gi|301112441|ref|XP_002997991.1| $37 . \overline{8} 0 \quad 82 \quad 50 \quad 1 \quad r \begin{array}{lllll}175 & 420 & 161\end{array}$

$2413 e-10 \quad 69.7$ ORF_isotig12422 gi|928498313|gb|KPD18813.1|

$\begin{array}{llllllllll}35.29 & 153 & 95 & 3 & 31 & 489 & 131 & 279 & 3 e-10 & 69.3\end{array}$

ORF_isotig12422 $\quad$ gi|672829171|gb|KFH74060.1| $38.53 \quad 109 \quad 56 \quad 4$

$190 \quad 504 \quad 488 \quad 589 \quad 3 e-10 \quad 70.5$ ORF_isotig12422

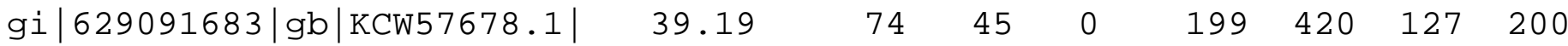

3e-10 68.9 ORF_isotig12422 gi|567155800|ref|XP_006418181.1|

$\begin{array}{llllllllll}35.56 & 90 & 58 & 0 & 151 & 420 & 75 & 164 & 4 \mathrm{e}-10 & 70.1\end{array}$

ORF_isotig12422 gi|494729398|ref|WP_007465264.1| $44.00 \quad 75 \quad 42$

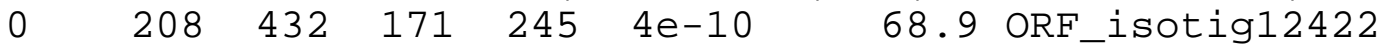

gi|296417443|ref|XP_002838367.1| $33.03 \quad 109 \quad 71 \quad 2 \quad 145 \quad 468 \quad 110$

$2174 \mathrm{e}-10 \quad 70.1$ ORF_isotig12422 gi|567155803|ref|XP_006418182.1|

$\begin{array}{llllllllll}35.56 & 90 & 58 & 0 & 151 & 420 & 111 & 200 & 4 \mathrm{e}-10 & 70.1\end{array}$

ORF_isotig12422 gi|727621889|ref|XP_010481782.1| $37.08 \quad 89 \quad 56$

○ $\quad 202468 \quad 128 \quad 216 \quad 4 \mathrm{e}-10 \quad 70.1$ ORF_isotig12422

gi|21104516|dbj|BAB93119.1| $33.02 \quad 0 \quad 106 \quad 71 \quad 000151 \quad 468 \quad 111 \quad 216$

4e-10 70.1 ORF_isotig12422 gi|219129430|ref|XP_002184892.1|

$\begin{array}{llllllllll}41.56 & 77 & 45 & 0 & 190 & 420 & 594 & 670 & 4 \mathrm{e}-10 & 70.5\end{array}$

ORF_isotig12422 gi|727535400|ref|XP_010441944.1| $37.08 \quad 89 \quad 56$

$\odot \quad 202 \quad 468 \quad 128 \quad 216 \quad 4 \mathrm{e}-10 \quad 70.1$ ORF_isotig12422

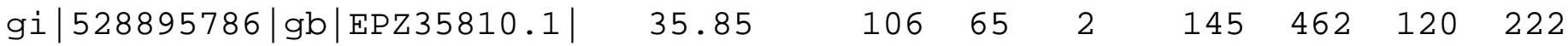

4e-10 70.1 ORF_isotig12422 gi|502110105|ref|XP_004493800.1|

$\begin{array}{llllllllll}42.25 & 71 & 41 & 0 & 208 & 420 & 129 & 199 & 4 \mathrm{e}-10 & 70.1\end{array}$

ORF_isotig12422 gi|743862914|ref|XP_010943667.1| $39.73 \quad 73 \quad 44$

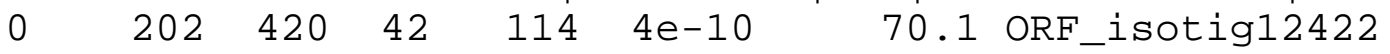

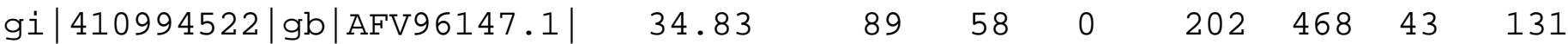

4e-10 66.6 ORF_isotig12422 gi|747088495|ref|XP_011091839.1|

$\begin{array}{llllllllll}38.96 & 77 & 47 & \odot & 190 & 420 & 124 & 200 & 5 \mathrm{e}-10 & 70.1\end{array}$

ORF_isotig12422 gi|695439924|ref|XP_009532833.1| $36.94 \quad 111 \quad 66$

$3 \quad 145 \quad 474 \quad 310 \quad 417 \quad 5 e-10 \quad 70.1 \quad$ ORF_isotig12422

gi|515351240|ref|WP_016863694.1| $36.36 \quad-\begin{array}{llllll}99 & 60 & 2 & 175 & 468 & 120\end{array}$

216 5e-10 69.7 ORF_isotig12422 gi|302789570|ref|XP_002976553.1|

$\begin{array}{llllllllll}37.65 & 85 & 53 & 0 & 202 & 456 & 128 & 212 & 5 \mathrm{e}-10 & 70.1\end{array}$

ORF_isotig12422 gi|545355774|ref|XP_005643662.1| $32.69 \quad 104 \quad 69$

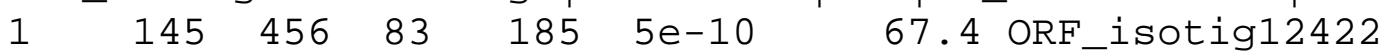

gi|390364326|ref|XP_003730583.1| $38.06 \quad-134 \quad 74 \quad 5 \quad 46 \quad 435 \quad 76$

$2045 e-10 \quad 67.8$ ORF_isotig12422 gi|743862910|ref|XP_010943666.1|

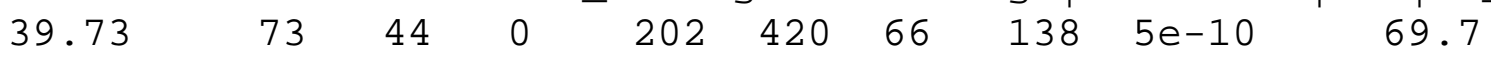

ORF_isotig12422 gi|659070963|ref|XP_008457484.1| $38.36 \quad 73 \quad 45$

( $\quad 202 \quad 420 \quad 233 \quad 305 \quad 5 e-10 \quad 69.3$ ORF_isotig12422

gi|19114464|ref|NP_593552.1| $28.70 \quad 108 \quad 76 \quad 1 \quad 145 \quad 468 \quad 145 \quad 251$

6e-10 69.7 ORF_isotig12422 gi|685358931|ref|XP_009114083.1|

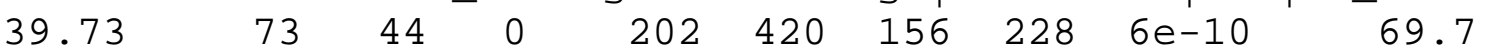

ORF_isotig12422 gi|748629167|ref|WP_039887344.1| $33.10 \quad 142 \quad 91$

$2 \quad 49 \quad 474 \quad 97 \quad 234 \quad 6 e-10 \quad 67.8$ ORF_isotig12422

gi|674920166|emb|CDY13130.1| $39.73 \quad 33 \quad 44 \quad 0 \quad 0202420 \quad 110 \quad 182$

6e-10 69.7 ORF_isotig12422 gi|302782732|ref|XP_002973139.1|

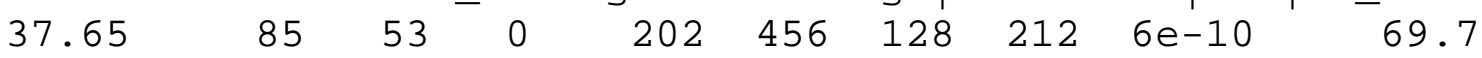

$\begin{array}{llllll}\text { ORF_isotig12422 } & \text { gi|758368534|emb|CEP } 99044.1 \mid & 45.00 & 60 & 33 & \odot\end{array}$

$253 \quad 432 \quad 2342936 \mathrm{e}-10 \quad 69.7$ ORF_isotig12422

gi|901821666|gb|KMZ73511.1| $31.52 \quad \begin{array}{lllllll}92 & 62 & 1 & 145 & 420 & 110 & 200\end{array}$

6e-10 69.7 ORF_isotig12422 gi|661175740|emb|CDH61092.1| 40.26

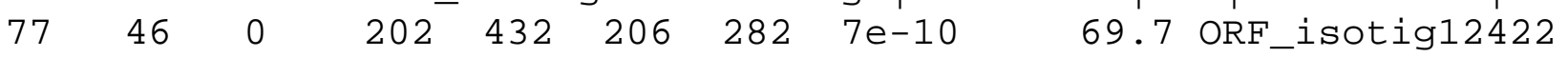

gi|743862902|ref|XP_010943664.1| $39.73 \quad 73 \quad 44 \quad 0 \quad 0202420 \quad 128$

200 7e-10 69.7 ORF_isotig12422 gi|18958247|dbj|BAB85602.1| 
35.16 $91 \quad 57$

1 202

$474 \quad 128 \quad 216 \quad 7 e-10$ 69.3

ORF_isotig12422 gi $\mid 472585716$ |gb|EMS23267.1| 34.06

$13864 \quad 4$

$19 \quad 432 \quad 107 \quad 217 \quad 7 \mathrm{e}-10 \quad 69.3 \quad$ ORF_isotig12422

gi|567902608|ref|XP_006443792.1| $39 . \overline{73} \quad 73 \quad 44 \quad 0 \quad 0202420 \quad 128$ $2007 \mathrm{e}-10 \quad 69.3$ ORF_isotig12422 gi|567902610|ref|XP_006443793.1|

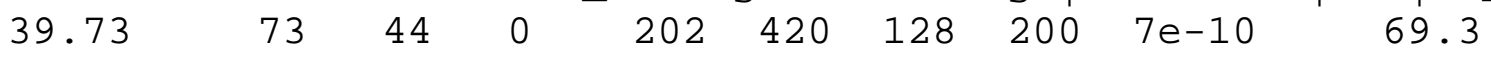

ORF_isotig12422 $\quad$ gi|674877470|emb|CDY54482.1| $35.16 \quad 91 \quad 57 \quad 1$ $202 \quad 474 \quad 128 \quad 216 \quad 7 \mathrm{e}-10$

gi|207100019| emb|CAK24968.2| $35.16 \quad-91 \quad 57 \quad 1$

7e-10 69.3 ORF_isotig12422 gi|672183480|ref|XP_008812020.1|

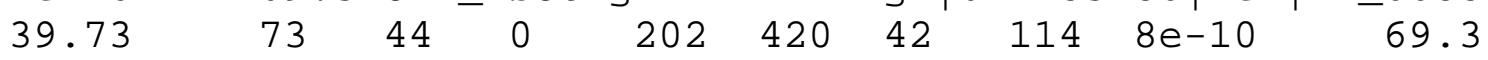

ORF_isotig12422 $\quad$ gi|641841508|gb|KD060420.1| $39.73 \quad 73 \quad 44 \quad 0$

$202 \quad 420 \quad 128 \quad 200 \quad 8 \mathrm{e}-10 \quad 69.3$ ORF_isotig12422

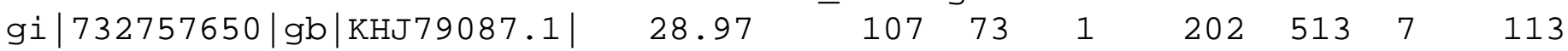

8e-10 65.5 ORF_isotig12422 gi|1005176715|gb|KYF56577.1| 44.59

$\begin{array}{lllllllll}74 & 37 & 1 & 253 & 474 & 150 & 219 & 8 \mathrm{e}-10 & 68.6\end{array}$

gi|659071562|ref|XP_008460630.1| $39.02 \quad 82 \quad 44 \begin{array}{lllll}1 & 202 & 429 & 235\end{array}$

316 8e-10 68.6 ORF_isotig12422 gi|333458192|gb|EGK86811.1|

$\begin{array}{llllllllll}33.10 & 142 & 91 & 2 & 49 & 474 & 127 & 264 & 8 \mathrm{e}-10 & 68.2\end{array}$

ORF_isotig12422 gi|255577926|ref|XP_002529835.1| $33.33 \quad 90 \quad 60$

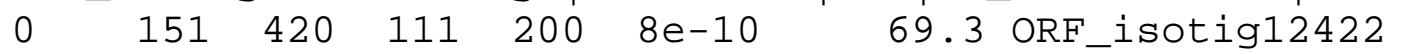

gi|694415952|ref|XP_009336118.1| $38.20 \quad-89 \quad 55 \quad 0 \quad 202 \quad 468 \quad 128$ 216 8e-10 69.3 ORF_isotig12422 gi|720020389|ref|XP_010262388.1|

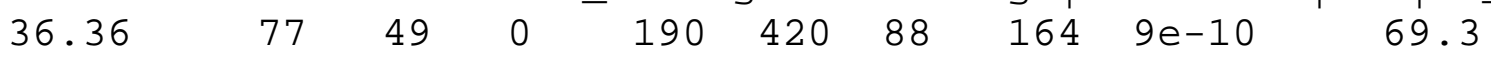

ORF_isotig12422 gi|694415950|ref|XP_009336117.1| $38.20 \quad 89 \quad 55$

$\odot \quad 202 \quad 468 \quad 140 \quad 228 \quad 9 \mathrm{e}-10 \quad 69.3$ ORF_isotig12422

gi|720020382|ref|XP_010262386.1| $36.36 \quad-77 \quad 49 \quad 0 \quad 190 \quad 420 \quad 124$ $2009 \mathrm{e}-10 \quad 69.3$ ORF_isotig12422 gi|723802071|ref|NP_001289777.1| $\begin{array}{llllllllll}36.36 & 77 & 49 & \odot & 190 & 420 & 124 & 200 & 9 \mathrm{e}-10 & 69.3\end{array}$

ORF_isotig12422 gi|915281149|ref|XP_013305271.1| 29.52 $105 \quad 73$

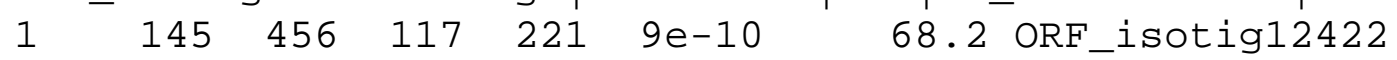

gi|255964729|gb|ACU44656.1| $34.44 \quad 090 \quad 59 \quad 00 \quad 151 \quad 420 \quad 111 \quad 200$

9e-10 69.3 ORF_isotig12422 gi|923847316|ref|XP_013702842.1|

$\begin{array}{llllllllll}33.02 & 106 & 71 & 0 & 151 & 468 & 111 & 216 & 9 \mathrm{e}-10 & 68.6\end{array}$

ORF_isotig12422 gi|672183478|ref|XP_008812019.1| $39.73 \quad 73 \quad 44$

○ $202 \quad 420 \quad 128 \quad 200 \quad 9 e-10 \quad 69.3$ ORF_isotig12422

gi|720020386|ref|XP_010262387.1| $36.36 \quad-77 \quad 49 \quad 0 \quad 190 \quad 420 \quad 124$ 200 9e-10 69.3 ORF_isotig12422 gi|46949222|gb|AAT07467.1|

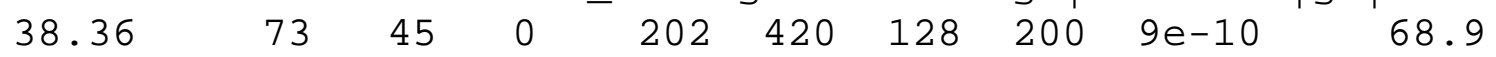

ORF_isotig12422 gi|760447765|ref|XP_011400951.1| $34.44 \quad 90 \quad 59$

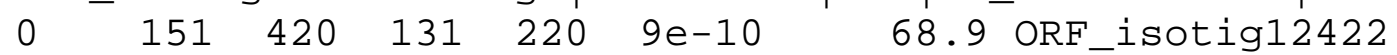

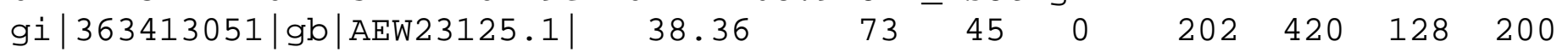

9e-10 68.9 ORF_isotig12422 gi|1000945507|ref|XP_015581239.1|

$\begin{array}{llllllllll}33.33 & 90 & 60 & 0 & 151 & 420 & 111 & 200 & 9 \mathrm{e}-10 & 68.9\end{array}$

ORF_isotig12422 $\quad$ gi|410994560|gb|AFV96166.1| $38.81 \quad 67 \quad 41 \quad \odot$

202402103169 1e-09 66.2 ORF_isotig12422

gi|923762135|ref|XP_013677346.1| $35.16 \quad 91 \quad 57 \quad 1 \quad 202 \quad 474 \quad 184$ 272 1e-09 68.9 ORF_isotig12422 gi|922533447|ref|XP_013598130.1|

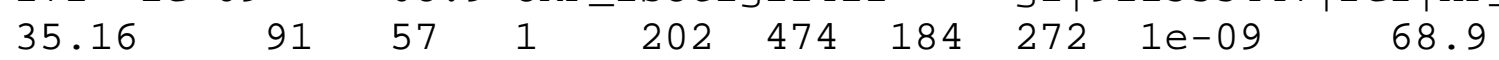

ORF_isotig12422 $\quad$ gi|220683835|gb|ACL80669.1| $39.73 \quad 73 \quad 44 \quad 0$

$202 \quad 420 \quad 128 \quad 200 \quad 1 \mathrm{e}-09 \quad 68.9$ ORF_isotig12422

gi|676382929|ref|XP_009034743.1| $42 . \overline{4} 7 \quad 73 \quad 42 \quad 0 \quad 214 \quad 432 \quad 142$ 214 1e-09 66.6 ORF_isotig12422 gi|923885023|ref|XP_013714028.1|

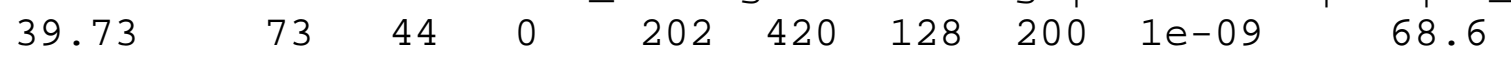

ORF_isotig12422 gi|764527045|ref|XP_011458022.1| $35.56 \quad 90 \quad 58$

$\odot \quad 199 \quad 468 \quad 131 \quad 220 \quad 1 \mathrm{e}-09 \quad 68.9$ ORF_isotig12422

gi|922563714|ref|XP_013610130.1| $39.73 \quad-73 \quad 44 \quad 0 \quad 202 \quad 420 \quad 130$ 
$2021 \mathrm{e}-\odot 9$

68.6 ORF_isotig12422

gi | 693266154 | gb|AIS24729.1|

$\begin{array}{llllllllll}41.10 & 73 & 41 & 1 & 256 & 474 & 188 & 258 & 1 \mathrm{e}-09 & 68.6\end{array}$

ORF_isotig12422

gi $|53760453| g b \mid$ AAU93349.1| 34.44

$90 \quad 59 \quad \odot$

$\begin{array}{lllll}151 & 420 & 111 & 200 & 1 \mathrm{e}-09\end{array}$

68.6 ORF_isotig12422

gi|873224759|emb|CEM25435.1| 34.62

$104 \quad 65 \quad 2$

$\begin{array}{llll}199 & 507 \quad 172 \quad 273\end{array}$

1e-09 68.9 ORF_isotig12422 gi|813208513|dbj|GA050417.1| 37.65

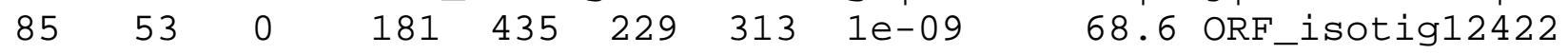

gi|470240016|ref|XP_004351977.1| $32.14 \quad 112 \quad 73 \quad 2 \quad 169 \quad 501 \quad 143$ 252 1e-09 68.6 ORF_isotig12422 gi|657981623|ref|XP_008382838.1|

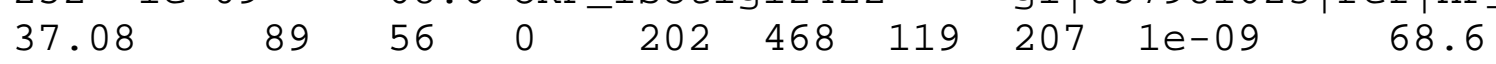

ORF_isotig12422 $\quad$ gi|674895889|emb|CDY36967.1| $39.73 \quad 73 \quad 44 \quad 0$

$202 \quad 420 \quad 128 \quad 200 \quad 2 e-09 \quad 68.2$ ORF_isotig12422

gi|802717229|ref|XP_012085275.1| $33.33 \quad 0000000151420 \quad 111$ 200 2e-09 68.6 ORF_isotig12422 gi|449445620|ref|XP_004140570.1| $\begin{array}{llllllllll}33.62 & 116 & 69 & 2 & 202 & 549 & 128 & 235 & 2 \mathrm{e}-09 & 68.6\end{array}$

ORF_isotig12422 gi|470107265|ref|XP_004289969.1| $35.56 \quad 90 \quad 58$

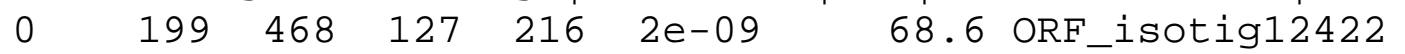

gi|302843401|ref|XP_002953242.1| $37.65 \quad-\begin{array}{llllll}85 & 53 & \odot & 181 & 435 & 125\end{array}$ 209 2e-09 66.6 ORF_isotig12422 gi|922548834|ref|XP_013602398.1| $\begin{array}{llllllllll}33.02 & 106 & 71 & 0 & 151 & 468 & 111 & 216 & 2 \mathrm{e}-09 & 68.2\end{array}$

ORF_isotig12422 gi|657961954|ref|XP_008372572.1| $37.08 \quad 89 \quad 56$

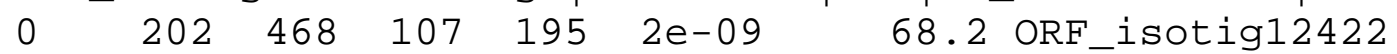

gi|966405784|ref|WP_058449190.1| $46.67 \quad-60 \quad 32 \quad 0 \quad 253 \quad 432 \quad 119$ 178 2e-09 65.9 ORF_isotig12422 gi|657981621|ref|XP_008382837.1| $\begin{array}{llllllllll}37.08 & 89 & 56 & 0 & 202 & 468 & 128 & 216 & 2 e-09 & 68.2\end{array}$

ORF_isotig12422 $\quad$ gi|113594574|dbj|BAF18448.1| $32.41 \quad 108 \quad 67 \quad 1$

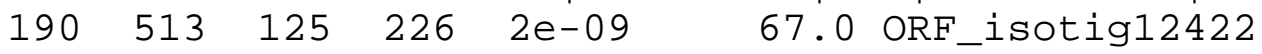

gi|731346789|ref|XP_010684640.1| $33.61 \quad 119 \quad 76 \quad 2 \quad 145 \quad 501 \quad 24$ 139 2e-09 68.2 ORF_isotig12422 gi|676430709|ref|XP_009046015.1| $\begin{array}{llllllllll}31.25 & 96 & 66 & \odot & 145 & 432 & 116 & 211 & 2 \mathrm{e}-09 & 66.6\end{array}$

ORF_isotig12422 gi|657961958|ref|XP_008372575.1| $37.08 \quad 89 \quad 56$

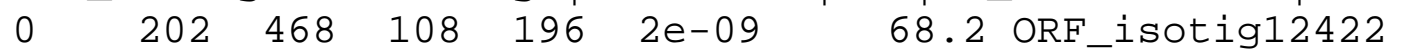

gi|729447939|ref|XP_010522567.1| $37.97 \quad-79 \quad 49 \quad 0 \quad 202 \quad 438 \quad 128$

206 2e-09 68.2 ORF_isotig12422 gi|923864531|ref|XP_013707638.1|

$\begin{array}{llllllllll}35.96 & 89 & 57 & 0 & 202 & 468 & 128 & 216 & 2 \mathrm{e}-09 & 68.2\end{array}$

ORF_isotig12422 gi|922429656|ref|XP_013620881.1| $35.96 \quad 89 \quad 57$

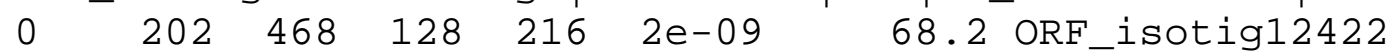

gi|294877972|ref|XP_002768219.1| $37.36 \quad-91 \quad 53 \quad 2 \quad 208 \quad 474 \quad 132$ 220 2e-09 67.8 ORF_isotig12422 gi|922429654|ref|XP_013620880.1| $\begin{array}{llllllllll}35.96 & 89 & 57 & 0 & 202 & 468 & 128 & 216 & 2 \mathrm{e}-09 & 68.2\end{array}$

ORF_isotig12422 gi|685383195|ref|XP_009123930.1| $35.96 \quad 89 \quad 57$

$\odot \quad 202468 \quad 128 \quad 216 \quad 2 \mathrm{e}-09 \quad 68.2$ ORF_isotig12422

gi|985450624|ref|XP_015386247.1| $38.36 \quad-73 \quad 45 \quad 0 \quad 02202420 \quad 92$ 164 2e-09 68.2 ORF_isotig12422 gi|731346783|ref|XP_010684637.1|

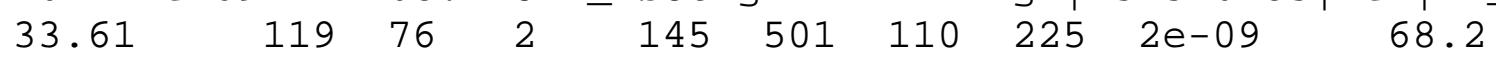

ORF_isotig12422

$205 \quad 420 \quad 129 \quad 200 \quad 2 \mathrm{e}-09 \quad 68.2$ ORF_isotig 12422

gi|224004956|ref|XP_002296129.1| $41.56 \quad 77 \quad 45 \quad 0 \quad 202 \quad 432 \quad 205$ 281 2e-09 67.8 ORF_isotig12422 gi|891585491|ref|XP_013022696.1| $\begin{array}{llllllllll}27.88 & 104 & 74 & 1 & 145 & 456 & 146 & 248 & 2 \mathrm{e}-09 & 67.8\end{array}$ ORF_isotig12422 gi|985450620|ref|XP_015386245.1| 38.36

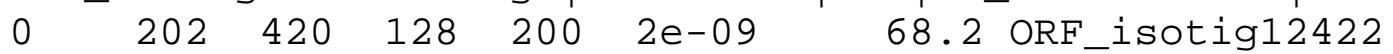

gi|731346787|ref|XP_010684639.1| $33.61 \quad 119 \quad 76 \quad 2 \quad 145 \quad 501 \quad 110$ 225 2e-09 67.8 ORF_isotig12422 gi|522072901|ref|WP_020584110.1|

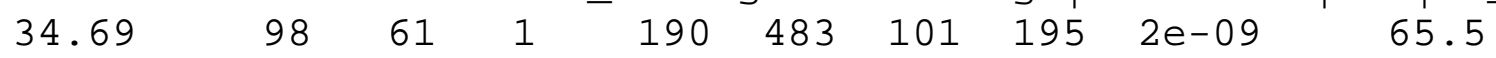

ORF_isotig12422 $\quad$ gi|641841507|gb|KD060419.1| $38.36 \quad 73 \quad 45 \quad 0$ $202420 \quad 126 \quad 198 \quad 2 \mathrm{e}-09 \quad 67.8$ ORF_isotig12422 
gi 674936482 | emb|CDX96939.1| 35.96

$\begin{array}{lllll}89 & 57 & 0 & 202 & 468\end{array}$

128216

2e-09 $\quad 67.8$ ORF_isotig12422

gi $\mid 922867416$ |gb|K0034056.1| 33.63

$\begin{array}{llllllll}113 & 73 & 1 & 100 & 432 & 125 & 237 & 2 \mathrm{e}-09\end{array}$

66.6 ORF_isotig12422

gi|551535405|ref|XP_005756498.1| 49.18

$\begin{array}{llllll}61 & 31 & 0 & 250 & 432 & 227\end{array}$

287 2e-09 67.4 ORF_isotig12422 gi|567902606|ref|XP_006443791.1|

$\begin{array}{llllllllll}38.36 & 73 & 45 & \odot & 202 & 420 & 128 & 200 & 2 \mathrm{e}-09 & 67.8\end{array}$

ORF_isotig12422 gi|676493293|ref|XP_009066193.1| $35.06 \quad 77 \quad 50$

$\odot \quad 190 \quad 420 \quad 119 \quad 195 \quad 3 e-09 \quad 65.9$ ORF_isotig12422

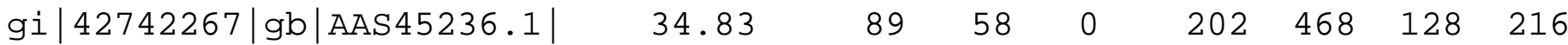

3e-09 67.8 ORF_isotig12422 gi|312072379|ref|XP_003139039.1|

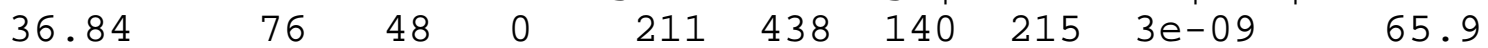

ORF_isotig12422 $\quad$ gi|407312484|gb|AFU06381.1| $32.26 \quad 124 \quad 75 \quad 3$

$145 \quad 513 \quad 110 \quad 225 \quad 3 e-09 \quad 67.8$ ORF_isotig12422

gi|657961548|ref|XP_008372367.1| $37.66 \quad 077 \quad 48 \quad 0 \quad 0202432 \quad 128$

204 3e-09 67.8 ORF_isotig12422 gi|536747464|gb|ERB64445.1|

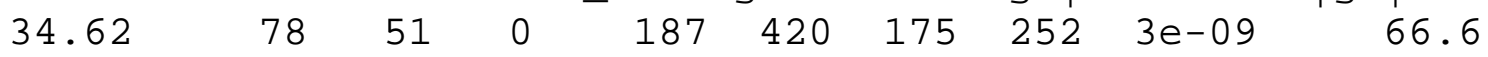

$\begin{array}{llllll}\text { ORF_isotig12422 } & \text { gi|473798304|gb|EMS46513.1| } & 32.41 & 108 & 67 & 1\end{array}$

$190 \quad 513 \quad 38 \quad 139 \quad 3 e-09 \quad 67.8$ ORF_isotig12422

gi|573946675|ref|XP_006655670.1| $31.40 \quad 121 \quad 77 \quad 1 \quad 151 \quad 513 \quad 113$

227 3e-09 67.8 ORF_isotig12422 gi|740321886|ref|WP_038159030.1|

$\begin{array}{llllllllll}34.62 & 78 & 51 & 0 & 187 & 420 & 175 & 252 & 3 e-09 & 66.6\end{array}$

$\begin{array}{lllll}\text { ORF_isotig12422 gi|970053982|ref|XP_015088621.1| } & 33.33 & 90 & 60\end{array}$

$\begin{array}{llllllll}0 & 151 & 420 & 111 & 200 & 3 e-09 & 67.8 & \text { ORF_isotig12422 }\end{array}$

gi|757790478|ref|WP_043008233.1| $34.62 \quad 078 \quad 51 \quad 0 \quad 0187420 \quad 175$

252 3e-09 66.6 ORF_isotig12422 gi|529161423|gb|AGS56990.1|

$\begin{array}{llllllllll}37.08 & 89 & 56 & \odot & 202 & 468 & 128 & 216 & 3 e-09 & 67.8\end{array}$

ORF_isotig12422 $\quad$ gi|565364522|ref|XP_006348973.1| $36.36 \quad 77 \quad 49$

$\begin{array}{llllllll}0 & 190 & 420 & 69 & 145 & 3 e-09 & 67.4 & \text { ORF_isotig12422 }\end{array}$

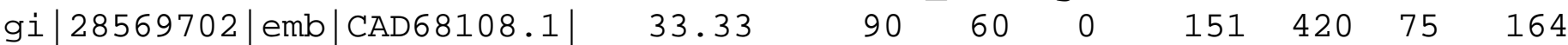

3e-09 67.8 ORF_isotig12422 gi|972777756|ref|NP_001305597.1|

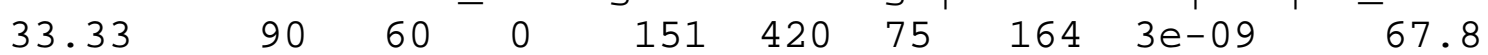

ORF_isotig12422 $\quad$ gi|444891679|gb|AGE13359.1| $41.56 \quad 77 \quad 45 \quad 0$

$202 \quad 432 \quad 205281 \quad 3 e-09 \quad 67.4$ ORF_isotig12422

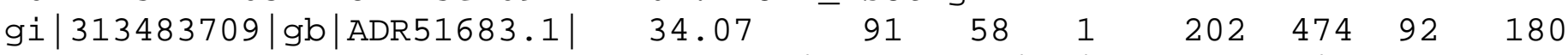

3e-09 67.8 ORF_isotig12422 gi|313483707|gb|ADR51682.1| 34.07

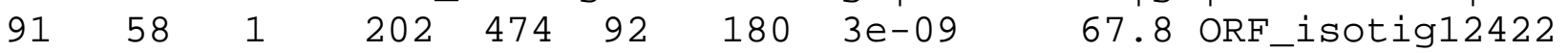

gi|567185112|ref|XP_006403145.1| $35.96 \quad 89 \quad 57 \quad 0 \quad 0202468 \quad 128$

216 3e-09 67.8 ORF_isotig12422 gi|568215457|ref|NP_001275308.1|

$\begin{array}{llllllllll}33.33 & 90 & 60 & \odot & 151 & 420 & 111 & 200 & 3 e-09 & 67.8\end{array}$

ORF_isotig12422 $\quad$ gi|373405317|gb|AEY68568.1| $36.26 \quad 91 \quad 56 \quad 1$

$202 \quad 474 \quad 128 \quad 216 \quad 3 e-09 \quad 67.8$ ORF_isotig12422

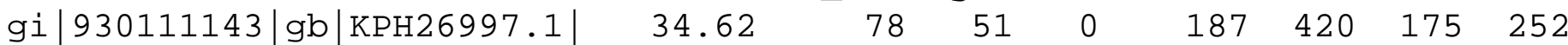

3e-09 66.2 ORF_isotig12422 gi|313483702|gb|ADR51680.1| 34.07

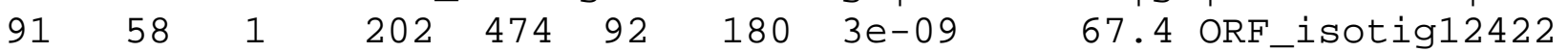

gi|740725877|ref|WP_038511163.1| $34.62 \quad 078 \quad 51 \quad 0 \quad 0187420 \quad 175$

252 3e-०9 66.2 ORF_isotig12422 gi|460404089|ref|XP_004247517.1|

$\begin{array}{llllllllll}33.33 & 90 & 60 & \odot & 151 & 420 & 111 & 200 & 3 e-09 & 67.8\end{array}$

ORF_isotig12422 $\quad$ gi|443690998|gb|ELT92982.1| $29.92 \quad 127 \quad 85 \quad 2$

$145 \quad 516 \quad 112 \quad 237 \quad 3 e-09 \quad 65.9$ ORF_isotig12422

gi|518106286|ref|WP_019276494.1| $34.62 \quad 078 \quad 51 \quad 0 \quad 187 \quad 420 \quad 175$

252 3e-09 66.2 ORF_isotig12422 gi|24963931|gb|AAG22095.3|

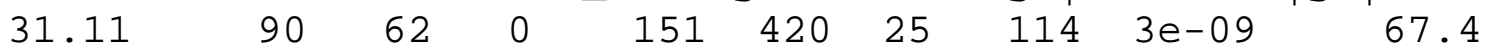

ORF_isotig12422 gi|494020115|ref|WP_006962411.1| $34.62 \quad 78 \quad 51$

$\odot \quad 187 \quad 420 \quad 175 \quad 252 \quad 3 e-09 \quad 66.2$ ORF_isotig12422

gi|970053984|ref|XP_015088622.1| $33.33 \quad-90 \quad 60 \quad 0 \quad 151 \quad 420 \quad 111$

200 3e-09 67.4 ORF_isotig12422 gi|723731779|ref|XP_010326652.1|

$\begin{array}{llllllllll}36.36 & 77 & 49 & \odot & 190 & 420 & 124 & 200 & 3 e-09 & 67.4\end{array}$ 
ORF_isotig12422

gi|659070971|ref|XP_008457528.1| 33.33

93

61

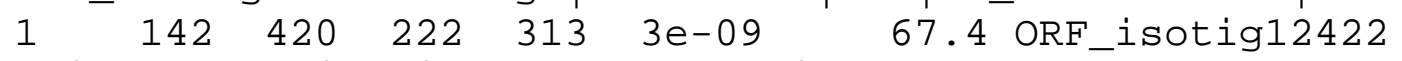

gi|743942813|ref|XP_011015906.1| $39.73 \quad-73 \quad 44 \quad 0 \quad 202 \quad 420 \quad 128$

200 3e-09 67.4 ORF_isotig12422 gi|685369694|ref|XP_009118681.1| $\begin{array}{llllllllll}33.02 & 106 & 71 & 0 & 151 & 468 & 111 & 216 & 4 \mathrm{e}-09 & 67.4\end{array}$

ORF_isotig12422 $\quad$ gi|674900498|emb|CDY32521.1| $33.02 \quad 106 \quad 71 \quad 0$

$\begin{array}{lllll}151 & 468 & 111 & 216 & 4 \mathrm{e}-09\end{array} 67.4$ ORF_isotig12422

gi|743869433|ref|XP_010905811.1| $35.37 \quad 82 \quad 51 \quad 1 \quad 175 \quad 420 \quad 35$

114 4e-09 67.0 ORF_isotig12422 gi|923703471|ref|XP_013660298.1|

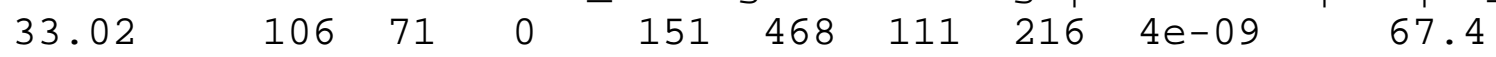

$\begin{array}{llllll}\text { ORF_isotig12422 } & \text { gi|166798217|gb|ABY89660.1| } & 34.44 & 9 \odot & 59 & \odot\end{array}$

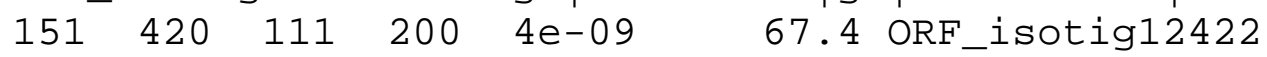

gi|590715534|ref|XP_007050223.1| $33.33 \quad 0000000151420 \quad 113$

202 4e-09 67.4 ORF_isotig12422 gi|218197390|gb|EEC79817.1|

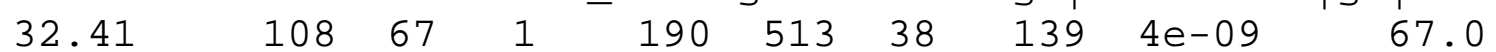

ORF_isotig12422 gi|747072284|ref|XP_011083048.1| $36.71 \quad 79 \quad 50$

$\odot \quad 202438 \quad 128 \quad 206 \quad 4 \mathrm{e}-09 \quad 67.4$ ORF_isotig12422

gi|939611289|gb|KPV73315.1| $47.46 \quad 090 \begin{array}{llllll}31 & 0 & 256 & 432 & 161 & 219\end{array}$

4e-09 67.0 ORF_isotig12422 gi|695064415|ref|XP_009420754.1|

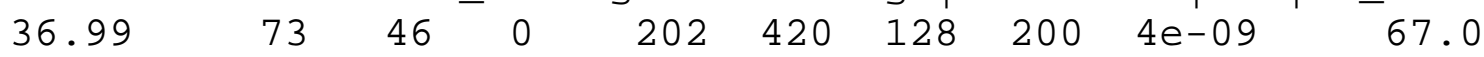

ORF_isotig12422 gi|685369692|ref|XP_009118680.1| $33.02 \quad 106 \quad 71$

$\begin{array}{lllllll}0 & 151 & 468 & 111 & 216 & 4 \mathrm{e}-09 & 67.0\end{array}$

ORF_comp2951_c3_seq1

gi $\mid 501733845$ |ref |WP_012629148.1| 39.31

145

$8125115 \quad 54944 \quad 1815 e-23 \quad 103$ ORF_comp2951_c3_seq1

gi|493029431|ref|WP_006099075.1| $38.73 \quad 14 \overline{2} 80 \quad 2 \quad \begin{array}{lllll}121 & 546 & 47\end{array}$ 181 1e-22 102 ORF_comp2951_c3_seq1 gi|740234212|ref|WP_038075400.1| $\begin{array}{llllllllll}41.10 & 146 & 77 & 4 & 115 & 546 & 36 & 174 & 2 \mathrm{e}-22 & 101\end{array}$

ORF_comp2951_c3_seq1 gi|648291774|ref|WP_026072923.1| 39.44

142

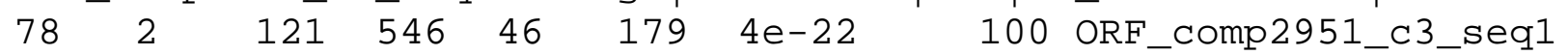

gi|501377640|ref|WP_012409206.1| $37.93 \quad 14 \overline{5} 82 \quad 4 \quad \begin{array}{lllll}115 & 546 & 41\end{array}$

178 9e-22 100 ORF_comp2951_c3_seq1 gi|505020123|ref|WP_015207225.1|

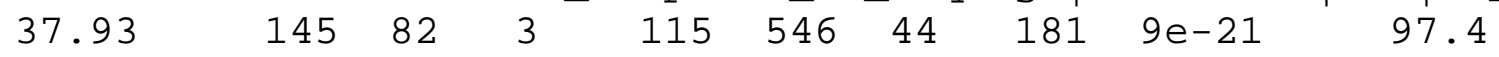

ORF_comp2951_c3_seq1 gi|738534441|ref|WP_036482850.1| 35.42

$90 \quad 2 \quad 118 \quad 546 \quad 46 \quad 187 \quad 1 e-20 \quad 97.1 \quad$ ORF_comp2951_c3_seq1

gi|658419581|ref|WP_029646255.1| $33.80 \quad 142 \quad 87 \quad 2 \quad \begin{array}{llll}121 & 546 & 47\end{array}$

181 1e-20 97.1 ORF_comp2951_c3_seq1

gi|522054471|ref|WP_020565680.1| 35.81

188 2e-20 96.7 ORF_comp2951_c3_seq1

gi|499931312|ref|WP_011612046.1| 37.93

180 2e-20 96.3 ORF_comp2951_c3_seq1

gi|515385832|ref|WP_016877723.1| 38.19

181 3e-20 95.5 ORF_comp2951_c3_seq1

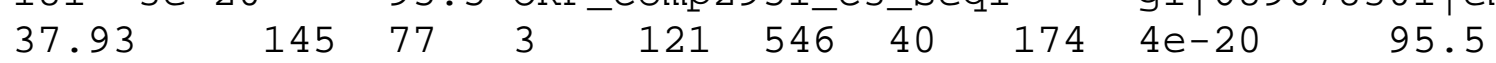

ORF_comp2951_c3_seq1 gi|656719681|gb|KEF42411.1| $38.78 \quad 147 \quad 80$

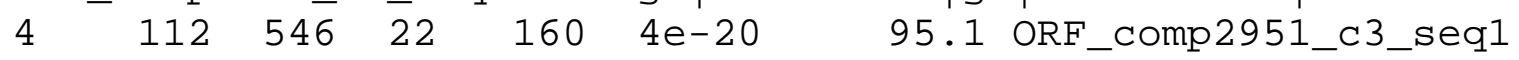

gi|981461217|ref|WP_059669889.1| 37.93

$1746 \mathrm{e}-20 \quad 94.7$ ORF_comp2951_c3_seq1

gi|763312982 |ref|WP_044171432.1| 37.32

172 7e-20 94.7 ORF_comp2951_c3_seq1

gi|826018244|ref|WP_047165229.1| 37.93

168 1e-19 94.4 ORF_comp2951_c3_seq1

gi $\mid 504925739$ |ref|WP_015112841.1| 38.46

181 2e-19 94.0 ORF_comp2951_c3_seq1

gi|516956783|ref|WP_018183684.1| 35.95

181 2e-19 93.6 ORF_comp2951_c3_seq1

$\begin{array}{llllll}145 & 77 & 3 & 121 & 546 & 40\end{array}$

$\begin{array}{llllll}142 & 82 & 2 & 121 & 546 & 38\end{array}$

$\begin{array}{llllll}145 & 80 & 3 & 115 & 546 & 33\end{array}$

$\begin{array}{llllll}143 & 80 & 3 & 121 & 546 & 46\end{array}$

$\begin{array}{llllll}153 & 88 & 4 & 121 & 579 & 39\end{array}$ 
gi|503088795 |ref|WP_013323642.1| 37.50

183 3e-19 93.2 ORF_comp2951_c3_seq1

gi|748165414|ref|WP_039738990.1| 36.36

$1814 \mathrm{e}-19$ 92.4 ORF_comp2951_c3_seq1

gi|515356232 |ref|WP_016865554.1| 37.24

180 6e-19 92.0 ORF_comp2951_c3_seq1 gi $\mid 941312503$ |ref |WP_055116231.1| 36.05

174 6e-19 92.0 ORF_comp2951_c3_seq1 gi|751277775|ref|WP_040986624.1| 36.11 172 7e-19 92.0 ORF_comp2951_c3_seq1 gi|751301901|ref|WP_041010414.1| 36.11 172 7e-19 92.0 ORF_comp2951_c3_seq1 gi|751301199|ref|WP_041009719.1| 36.11 172 8e-19 91.7 ORF_comp2951_c3_seq1 $\begin{array}{llllllll}36.11 & 144 & 85 & 3 & 115 & 546 & 36 & 172\end{array}$ ORF_comp2951_c3_seq1 gi| 751264288 |ref $\begin{array}{lllllll}85 & 3 & 115 & 546 & 38 & 174 & 8 \mathrm{e}-19\end{array}$ gi|737933697|ref|WP_035898371.1| 36.05 174 9e-19 91.7 ORF_comp2951_c3_seq1 gi |648412694|ref|WP_026104445.1| 35.66 182 1e-18 91.7 ORF_comp2951_c3_seq1 gi |917776332|ref|WP_052290273.1| 36.11 169 2e-18 90.9 ORF_comp2951_c3_seq1

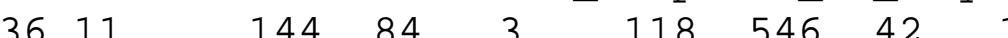
ORF_comp2951_c3_seq1 118 546 $42 \quad 178 \quad 2 \mathrm{e}-18$ gi|927917924|emb|CDX53272.1| 36.11

$3 \quad 115 \quad 546 \quad 36 \quad 172 \quad 2 \mathrm{e}-18 \quad 90.9$ ORF_comp2951_c3_seq1 gi|652338179|ref|WP_026735392.1| 36.55 $1812 \mathrm{e}-18$ 90.9 ORF_comp2951_c3_seq1 gi $\mid 493165631$ |ref |WP_006170876.1| 39.04 207 3e-18 90.5 ORF_comp2951_c3_seq1 gi $504945810 \mid$ ref |WP_015132912.1| 35.92 $1804 \mathrm{e}-18$ 89.7 ORF_comp2951_c3_seq1 gi|505018197|ref|WP_015205299.1| 37.76 $1814 \mathrm{e}-18$ 89.7 ORF_comp2951_c3_seq1

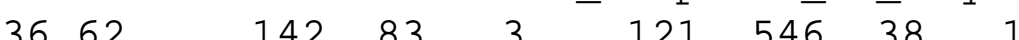

ORF_comp2951_c3_seq1 gi|953510847|dbj|BAT53535.1| 35.62

$\begin{array}{llllll}145 & 84 & 4 & 115 & 546 & 44\end{array}$

$\begin{array}{llllll}146 & 81 & 3 & 112 & 546 & 69\end{array}$

$\begin{array}{llllll}142 & 83 & 3 & 121 & 546 & 47\end{array}$

$\begin{array}{llllll}143 & 81 & 3 & 121 & 546 & 46\end{array}$ gi $|814343774| \mathrm{emb}|\mathrm{CPH} 68122.1|$ $5 \mathrm{e}-18 \quad 89.7$

$5 \quad 115546 \quad 36 \quad 1735 \mathrm{e}-18$ 89.4 ORF_comp2951_c3_seq1 gi $|916294818|$ ref |WP_051029864.1| 36.99 179 5e-18 89.4 ORF_comp2951_c3_seq1 gi|737172391|ref|WP_035158595.1| 36.36 178 5e-18 89.4 ORF_comp2951_c3_seq1 gi|821561551|ref|WP_046868556.1| 37.32 175 6e-18 89.4 ORF_comp2951_c3_seq1 gi|497576287|ref|WP_009890471.1| 37.06 174 6e-18 89.4 ORF_comp2951_c3_seq1 $\begin{array}{llllllll}37.06 & 143 & 81 & 4 & 121 & 546 & 38\end{array}$

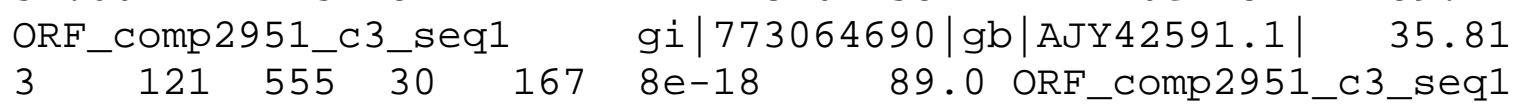
gi|773064690|gb|AJY42591.1| 35.81

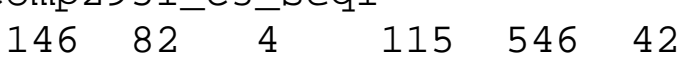
$\begin{array}{llllll}143 & 83 & 4 & 121 & 546 & 43\end{array}$ $\begin{array}{llllll}142 & 82 & 3 & 121 & 546 & 41\end{array}$ $\begin{array}{llllll}143 & 81 & 4 & 121 & 546 & 40\end{array}$ gi | 584107904 | gb|AHI79522.1| 6e-18 89.4 gi | 495550750|ref |WP_-008275329.1 | 37.06 177 8e-18 89.0 ORF_comp2951_c3_seq1 gi|499442921|ref|WP_011130385.1| 38.89 187 8e-18 89.0 ORF_comp2951_c3_seq1 gi|492895672|ref|WP_006026078.1| 35.81 177 9e-18 89.0 ORF_comp2951_c3_seq1 gi|756829253|ref|WP_042646352.1| 35.42 172 9e-18 89.0 ORF_comp2951_c3_seq1 gi|981645748|ref|WP_059844321.1| 37.06

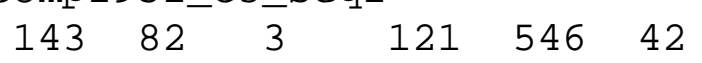

$\begin{array}{llllll}144 & 78 & 5 & 121 & 546 & 52\end{array}$

$\begin{array}{llllll}148 & 82 & 3 & 121 & 555 & 40\end{array}$

$\begin{array}{llllll}144 & 86 & 3 & 115 & 546 & 36\end{array}$

$\begin{array}{llllll}143 & 81 & 4 & 121 & 546 & 40\end{array}$ 
174 1e-17 88.6 ORF_comp2951_c3_seq1 gi|754360430|ref|WP_041861630.1| 35.86 174 1e-17 88.2 ORF_comp2951_c3_seq1 $\begin{array}{lllllll}35.86 & 145 & 80 & 3 & 121 & 546 & 30\end{array}$ $\begin{array}{llllll}145 & 80 & 3 & 121 & 546 & 40\end{array}$ gi | 484585464 | gb |AGK47089 . 1 | ORF_comp2951_c3_seq1 gi |504891436 |ref |WP_015078538.1| 34.48 $87 \quad 3 \quad 115 \quad 546 \quad 44 \quad 181 \quad 1 e-17 \quad 88.2$ ORF_comp2951_c3_seq1 gi|504949901|ref|WP_015137003.1| 37.41 185 1e-17 88.2 ORF_comp2951_c3_seq1 $34.72 \quad 144 \quad 84 \quad 5$
ORF_comp2951_c3_seq1 $\begin{array}{lllll}121 & 546 & 38 & 173 & 2 \mathrm{e}-17\end{array}$ $\begin{array}{llllll}147 & 83 & 4 & 121 & 555 & 46\end{array}$ gi | 75702893 | gb |ABA22569.1| gi|981383968|ref|WP_059597148.1| 37.24 145

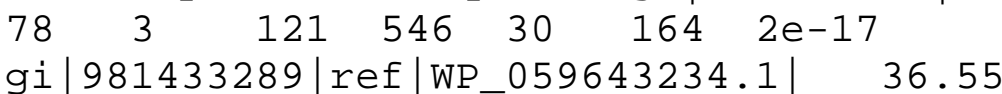
174 2e-17 88.2 ORF_comp2951_c3_seq1 gi|504970487|ref|WP_015157589.1| 35.66 175 2e-17 87.8 ORF_comp2951_c3_seq1 gi|981741072|ref|WP_059934588.1| 35.86 174 2e-17 87.8 ORF_comp2951_c3_seq1

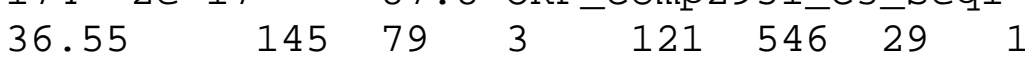
88.2 ORF_comp2951_c3_seq1

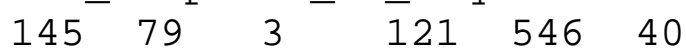

$\begin{array}{llllll}143 & 84 & 3 & 121 & 546 & 40\end{array}$

$\begin{array}{llllll}145 & 80 & 3 & 121 & 546 & 40\end{array}$ gi $\mid 705475049$ |gb|KGSO4910.1| $2 \mathrm{e}-17 \quad 87.8$

ORF_comp2951_c3_seq1 gi|740960548|ref|WP_038745121.1| 36.55

$\begin{array}{lllllll}79 & 3 & 121 & 546 & 40 & 174 & 2 \mathrm{e}-17\end{array} 87.8$ ORF_comp2951_c3_seq1

gi|764950513|ref|WP_044520790.1| 34.03

173 3e-17 87.4 ORF_comp2951_c3_seq1 gi|505013627|ref|WP_015200729.1| 34.03 181 3e-17 87.4 ORF_comp2951_c3_seq1

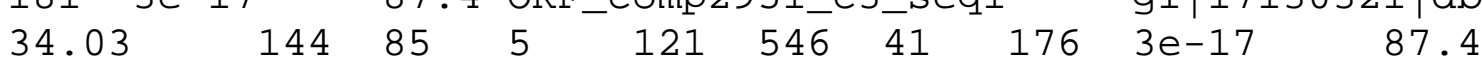
ORF_comp2951_c3_seq1 gi|504967338|ref|WP_015154440.1| 34.27 $86 \quad 3 \quad 121 \quad 546 \quad 45 \quad 180 \quad 3 e-17 \quad 87.4$ ORF_comp2951_c3_seq1 gi|657935844 |ref |WP_029636980.1| 34.97 $\begin{array}{llrl}181 & 3 e-17 & 87.4 & \text { ORF_comp2951_c3_seq1 } \\ \text { gi }|740984489| \text { ref |WP } & \text { O38768164.1| } 36.55\end{array}$ 174 3e-17 87.0 ORF_comp2951_c3_seq1

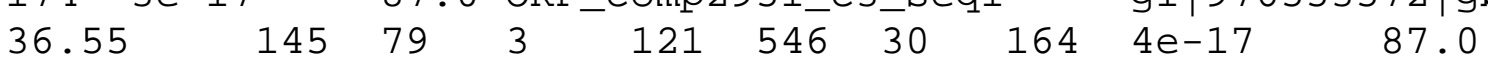
ORF_comp2951_c3_seq1 gi|976471317|gb|KVD74754.1| 36.55 $143 \quad 85 \quad 4 \quad 121 \quad 546 \quad 46$ $\begin{array}{llllll}145 & 79 & 3 & 121 & 546 & 40\end{array}$ gi | 976553572 |gb|KVE52227.1| $\begin{array}{llllll}144 & 87 & 3 & 118 & 546 & 45\end{array}$ gi|17130321|dbj |BAB72932.1| $144 \quad 85 \quad 5 \quad 121 \quad 546 \quad 38$ $3 \quad 121546 \quad 30 \quad 164$ 4e-17 87.0 ORF_comp2951_c3_seq1 gi|909830227|gb|AKU13025.1| $35.21 \quad 142 \quad 85 \quad 3 \quad \begin{array}{lllll}121 & 546 & 45 & 179\end{array}$ 4e-17 87.0 ORF_comp2951_c3_seq1 gi|913438795|ref|WP_050418395.1|

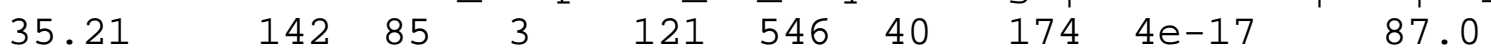
ORF_comp2951_c3_seq1 gi|497590677|ref|WP_009904861.1| 35.86 $80 \quad 3 \quad 121 \quad 546 \quad 40 \quad 174 \quad 4 \mathrm{e}-17 \quad 87.0$ ORF_comp2951_c3_seq1 gi|782673738|ref|WP_045600604.1| 35.86 174 4e-17 87.0 ORF_comp2951_c3_seq1 gi|497231444|ref|WP_009545706.1| 35.66 177 6e-17 86.3 ORF_comp2951_c3_seq1 gi|981251097|ref|WP_059470554.1| 36.55 173 9e-17 85.9 ORF_comp2951_c3_seq1 gi|656037395|ref|WP_029076335.1| 32.68 181 1e-16 85.9 ORF_comp2951_c3_seq1 gi|515888082|ref|WP_017318665.1| 34.27 181 1e-16 85.9 ORF_comp2951_c3_seq1 gi|974600092|ref|WP_059190108.1| 34.03 172 2e-16 85.1 ORF_comp2951_c3_seq1 gi|913356948|ref|WP_050376444.1| 35.86 174 2e-16 85.1 ORF_comp2951_c3_seq1 $\begin{array}{llllll}145 & 80 & 3 & 121 & 546 & 40\end{array}$ $\begin{array}{llllll}143 & 84 & 3 & 121 & 546 & 42\end{array}$ $\begin{array}{llllll}145 & 79 & 3 & 121 & 546 & 39\end{array}$ $\begin{array}{llllll}153 & 93 & 4 & 121 & 579 & 39\end{array}$

$\begin{array}{llllll}143 & 86 & 3 & 121 & 546 & 46\end{array}$

$\begin{array}{llllll}144 & 88 & 3 & 115 & 546 & 36\end{array}$

$\begin{array}{llllll}145 & 80 & 3 & 121 & 546 & 40\end{array}$ gi|160698859|gb|EDP88829.1| $\begin{array}{llllllllll}35.86 & 145 & 80 & 3 & 121 & 546 & 136 & 270 & 2 \mathrm{e}-16 & 85.5\end{array}$ ORF_comp2951_c3_seq1 gi|490301683|ref|WP_004197081.1| 35.86 $80 \quad 3 \quad 121 \quad 546 \quad 40 \quad 174 \quad 2 \mathrm{e}-16 \quad 84.7$ ORF_comp2951_c3_seq1 gi|953804284|ref|WP_058039816.1| $35.86 \quad 145 \quad 80 \quad 3 \quad \begin{array}{lllll}121 & 546 & 40\end{array}$ 
174 2e-16 84.7 ORF_comp2951_c3_seq1

gi|740959588|ref|WP_038744210.1| 35.86

174 2e-16 84.7 ORF_comp2951_c3_seq1

$\begin{array}{llllll}145 & 80 & 3 & 121 & 546 & 40\end{array}$

gi $\mid 148027253$ |gb|EDK85274.1|

$\begin{array}{llllllllll}35.86 & 145 & 80 & 3 & 121 & 546 & 140 & 274 & 2 \mathrm{e}-16 & 85.5\end{array}$

ORF_comp2951_c3_seq1 gi|243063500|gb|EES45686.1| $35.86 \quad 145 \quad 80$

$3 \quad 121546 \quad 140 \quad 274 \quad 2 \mathrm{e}-16 \quad 85.5$ ORF_comp2951_c3_seq1

gi|497606907|ref|WP_009921091.1| $35.86 \quad 145 \quad 80 \quad 3 \quad 121 \quad 546 \quad 40$

174 2e-16 84.7 ORF_comp2951_c3_seq1 gi|121227363|gb|ABM49881.1|

$\begin{array}{llllllllll}35.86 & 145 & 80 & 3 & 121 & 546 & 142 & 276 & 2 \mathrm{e}-16 & 85.5\end{array}$

ORF_comp2951_c3_seq1 $\quad$ gi|800785064|emb|CFL25926.1| $35.86 \quad 145 \quad 80$

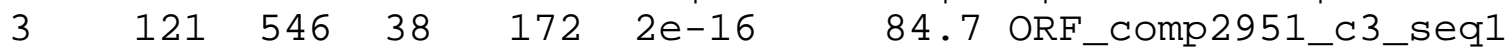

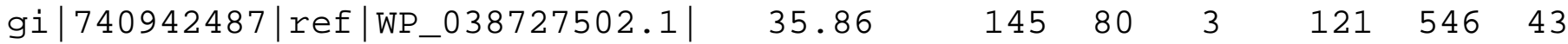

177 2e-16 84.7 ORF_comp2951_c3_seq1

gi|752526330|ref|WP_041197894.1| 35.86

174 3e-16 84.7 ORF_comp2951_c3_seq1

gi|740980945|ref|WP_038764816.1| 35.86

177 3e-16 84.7 ORF_comp2951_c3_seq1

gi $|752524486|$ ref |WP_041196068.1| 35.86

174 3e-16 84.7 ORF_comp2951_c3_seq1

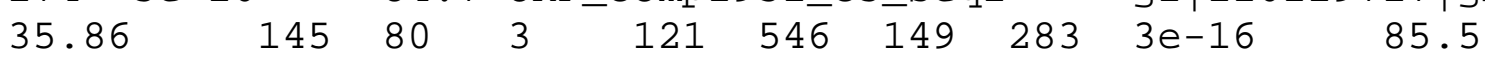

ORF_comp2951_c3_seq1 gi|953803876|ref|WP_058039691.1| 35.86

$80 \quad 3 \quad 121 \quad 546 \quad 40 \quad 174 \quad 3 e-16 \quad 84.7$ ORF_comp2951_c3_seq1

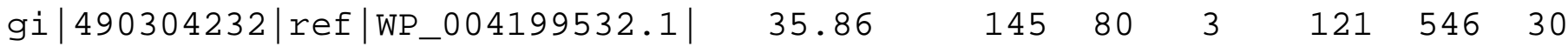

164 3e-16 84.3 ORF_comp2951_c3_seq1 gi|490685647|ref|WP_004550267.1| 35.86

172 3e-16 84.7 ORF_comp2951_c3_seq1

$\begin{array}{llllllllll}35.86 & 145 & 80 & 3 & 121 & 546 & 151 & 285 & 3 e-16 & 85.5\end{array}$

ORF_comp2951_c3_seq1 $\quad$ gi|686840559|gb|KGC20015.1| $35.86 \quad 145 \quad 80$

$3 \quad 121 \quad 546 \quad 30 \quad 164$ 3e-16 84.3 ORF_comp2951_c3_seq1

gi|714526348|gb|KGV56958.1| $35.86 \quad 145 \quad 80 \quad 3 \quad \begin{array}{lllll}121 & 546 & 38 & 172\end{array}$

3e-16 84.7 ORF_comp2951_c3_seq1 gi|714646342|gb|KGW52040.1|

$\begin{array}{llllllllll}35.86 & 145 & 80 & 3 & 121 & 546 & 30 & 164 & 3 e-16 & 84.3\end{array}$

ORF_comp2951_c3_seq1 gi|981296166|ref|WP_059513522.1| 35.21

$85 \quad 3 \quad 121 \quad 546 \quad 39 \quad 173 \quad 3 e-16 \quad 84.7$ ORF_comp2951_c3_seq1

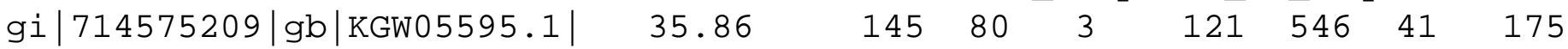

3e-16 84.7 ORF_comp2951_c3_seq1 gi|741015566|ref |WP_038797782.1|

$\begin{array}{llllllllll}35.86 & 145 & 80 & 3 & 121 & 546 & 40 & 174 & 3 e-16 & 84.7\end{array}$

ORF_comp2951_c3_seq1 gi|714507186|gb|KGV37859.1| $35.86 \quad 145 \quad 80$

$3 \quad 121546 \quad 30 \quad 164$ 3e-16 84.3 ORF_comp2951_c3_seq1

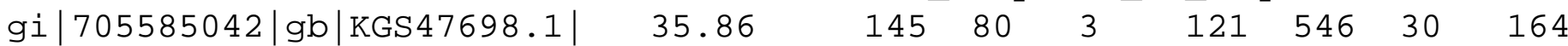

3e-16 84.3 ORF_comp2951_c3_seq1 gi|759580794|ref|WP_043299684.1|

$\begin{array}{llllllllll}35.86 & 145 & 80 & 3 & 121 & 546 & 40 & 174 & 3 e-16 & 84.7\end{array}$

ORF_comp2951_c3_seq1 $\quad$ gi|126229014|gb|ABN92554.1| $35.86 \quad 145 \quad 80$

$3 \quad 121546 \quad 145 \quad 279$ 3e-16 85.5 ORF_comp2951_c3_seq1

gi|686848709|gb|KGC28119.1| $35.86 \quad 145 \quad 80 \quad 3 \quad 121 \quad 546 \quad 30 \quad 164$

3e-16 84.3 ORF_comp2951_c3_seq1 gi|169654349|gb|EDS87042.1|

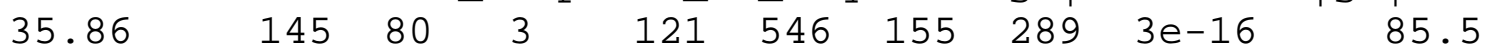

ORF_comp2951_c3_seq1 gi|157936951|gb|ED092621.1| $35.86 \quad 145 \quad 80$

$3 \quad 121546 \quad 160 \quad 294 \quad 3 e-16 \quad 85.5$ ORF_comp2951_c3_seq1

gi|701274860|gb|AIV79086.1| $35.86 \quad 145 \quad 80 \quad 3 \quad$ 121 $546 \quad 30 \quad 164$

3e-16 84.3 ORF_comp2951_c3_seq1 gi|254218068|gb|EET07452.1|

$\begin{array}{llllllllll}35.86 & 145 & 80 & 3 & 121 & 546 & 162 & 296 & 3 e-16 & 85.5\end{array}$

ORF_comp2951_c3_seq1 gi|217395731|gb|EEC35749.1| $35.86 \quad 145 \quad 80$

$3 \quad 121546 \quad 168 \quad 302 \quad 3 e-16 \quad 85.5$ ORF_comp2951_c3_seq1

gi|741018909|ref|WP_038800916.1| $35.92 \quad 3 \quad 142 \quad 84 \quad 3 \quad 121 \quad 546 \quad 39$

173 4e-16 84.3 ORF_comp2951_c3_seq1 gi|685679385|gb|AI066505.1|

$\begin{array}{llllllllll}35.92 & 142 & 84 & 3 & 121 & 546 & 38 & 172 & 4 \mathrm{e}-16 & 84.3\end{array}$ 
ORF_comp2951_c3_seq1

gi|157806771|gb|ED083941.1| 35.86

14580

$3 \quad 121546 \quad 158 \quad 292$ 4e-16 85.1 ORF_comp2951_c3_seq1

gi|225927831|gb|EEH23872.1| 35.86

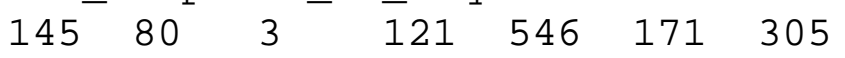

4e-16 85.5 ORF_comp2951_c3_seq1

gi $\mid 134251017$ | gb|EBA51096.1|

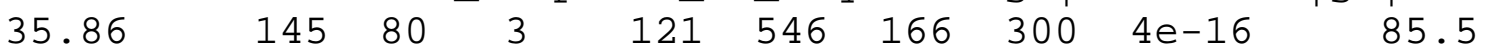

ORF_comp2951_c3_seq1 gi|237502694|gb|ACQ95012.1| 35.86

$3 \quad 121546 \quad 144 \quad 278$ 4e-16 85.1 ORF_comp2951_c3_seq1

gi|714902087|gb|KGX30928.1| $35.86 \quad 145 \quad 80 \quad 3 \quad \begin{array}{lllll}121 & 546 & 152 & 286\end{array}$

4e-16 85.1 ORF_comp2951_c3_seq1 gi|640368848|ref|WP_024880427.1|

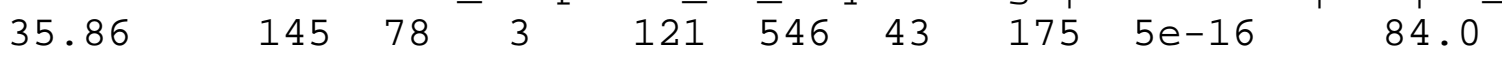

ORF_comp2951_c3_seq1 gi|85544315|pdb|2BTW|B $33.33 \quad 144 \quad 86 \quad 5$

12154652187 6e-16 84.0 ORF_comp2951_c3_seq1

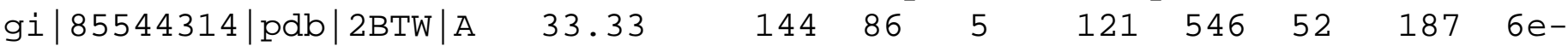

16 83.6 ORF_comp2951_c3_seq1 gi|817516937|ref|WP_046572938.1|

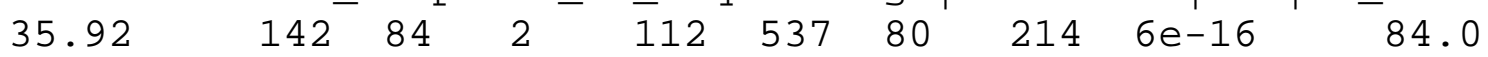

ORF_comp2951_c3_seq1 gi|751564302|ref|WP_041033204.1| 33.57

143

$87 \quad 3 \quad 121 \quad 546 \quad 46 \quad 181 \quad 7 \mathrm{e}-16 \quad 83.2$ ORF_comp2951_c3_seq1

gi|741007236|ref|WP_038789710.1| $35.17 \quad 145 \quad 81 \quad 3 \quad \begin{array}{llll}121 & 546 & 40\end{array}$

174 8e-16 83.2 ORF_comp2951_c3_seq1

gi|741019975|ref|WP_038801977.1| 35.86

173 8e-16 83.2 ORF_comp2951_c3_seq1

$\begin{array}{llllll}145 & 80 & 3 & 121 & 546 & 39\end{array}$

gi|772972989|gb|AJX33047.1|

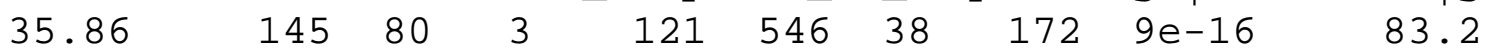

ORF_comp2951_c3_seq1 gi|740943421|ref|WP_038728427.1| 35.17

$81 \quad 3 \quad 121 \quad 546 \quad 40 \quad 174$ 9e-16 83.2 ORF_comp2951_c3_seq1

145

gi|785594494|ref|WP_045719475.1| $35.17 \quad 145 \quad 81 \quad 3 \quad 121 \quad 546 \quad 40$

174 9e-16 83.2 ORF_comp2951_c3_seq1 gi|806494169|gb|KKC16091.1|

$\begin{array}{llllllllll}35.17 & 145 & 81 & 3 & 121 & 546 & 30 & 164 & 9 \mathrm{e}-16 & 82.8\end{array}$

ORF_comp2951_c3_seq1 gi|938273386|gb|KPQ๑8481.1| $35.21 \quad 142 \quad 85$

$3 \quad 121 \quad 546 \quad 32 \quad 166 \quad 1 e-15 \quad 82.8$ ORF_comp2951_c3_seq1

gi|123963072|gb|ABM77828.1| $36.11 \quad 144 \quad 82 \quad 5 \quad \begin{array}{lllll}121 & 546 & 52 & 187\end{array}$

1e-15 83.2 ORF_comp2951_c3_seq1 gi|496440563|ref|WP_009149408.1|

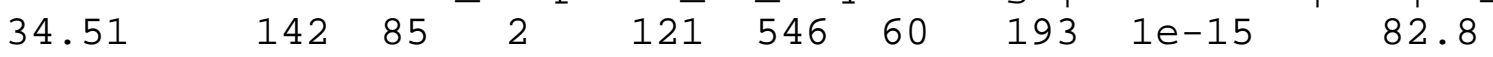

ORF_comp2951_c3_seq1 gi|740986897|ref|WP_038770440.1| 35.17

$8133121 \quad 546 \quad 40 \quad 174$ 1e-15 82.4 ORF_comp2951_c3_seq1

gi|568795567|gb|AHE26011.1| $35.17 \quad 345 \quad 81 \quad 3 \quad 121 \quad 546 \quad 30$

1e-15 82.4 ORF_comp2951_c3_seq1 gi|427346779|gb|AFY29492.1|

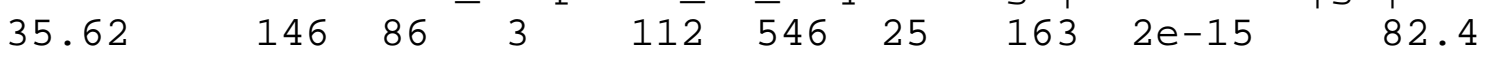

ORF_comp2951_c3_seq1 gi|652914138|ref|WP_027168079.1| 33.33

$89 \quad 3 \quad 115 \quad 546 \quad 36 \quad 172 \quad 2 \mathrm{e}-15 \quad 82.4$ ORF_comp2951_c3_seq1

gi|686936961|gb|KGD15055.1| $37.04 \quad 135 \quad 72 \quad 3 \quad 151 \quad \overline{5} 46 \quad 8 \quad 132$

2e-15 81.6 ORF_comp2951_c3_seq1 gi|740967447|ref |WP_038751917.1|

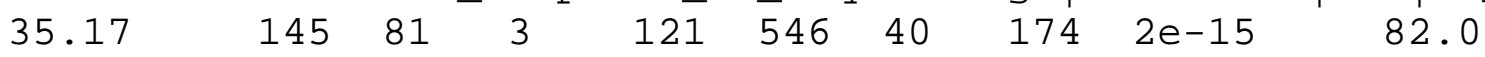

ORF_comp2951_c3_seq1 gi|759607566|ref|WP_043325766.1| 35.62

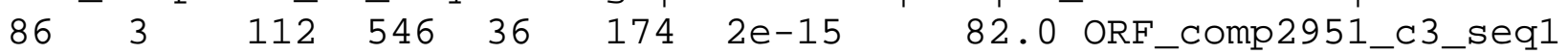

gi|738342341|ref|WP_036294947.1| $35.17 \quad 145 \quad 79 \quad 3 \quad r \begin{array}{llll}121 & 546 & 48\end{array}$

180 3e-15 81.6 ORF_comp2951_c3_seq1

gi|737586499|ref|WP_035557439.1| 35.92

227 3e-15 82.0 ORF_comp2951_c3_seq1

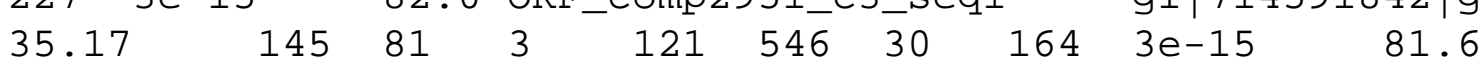

ORF_comp2951_c3_seq1 gi|501395618|ref|WP_012427184.1| 37.32

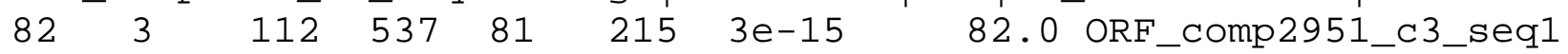

gi|651604058|ref|WP_026598946.1| $35.17 \quad 145 \quad 79 \quad 3 \quad \begin{array}{llll}121 & 546 & 38\end{array}$

$1704 \mathrm{e}-15$ 81.3 ORF_comp2951_c3_seq1 gi|814476642|emb|CPI32267.1|

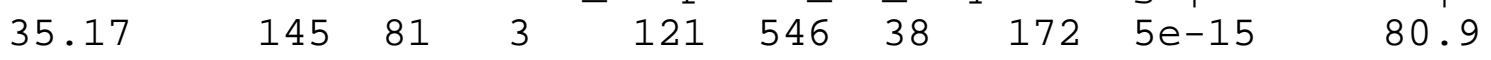

ORF_comp2951_c3_seq1 gi|648499655|ref|WP_026191406.1| 35.17

$79 \quad 3 \quad 121 \quad 546 \quad 43 \quad 175$ 5e-15 80.9 ORF_comp2951_c3_seq1

gi|917319449|ref|WP_051926161.1| $35.71 \quad 140 \quad 82 \quad 3 \quad \begin{array}{lllll}121 & 537 & 46\end{array}$ 
178 7e-15 80.5 ORF_comp2951_c3_seq1

gi $\mid 494317175$ |ref|WP_007177410.1| 35.92

$2247 e-15 \quad 80.9$ ORF_comp2951_c3_seq1

$\begin{array}{llllllll}34.67 & 150 & 75 & 3 & 112 & 537 & 76 & 210\end{array}$

gi $\mid 928498313$ |gb|KPD18813.1|

ORF_comp2951_c3_seq1 gi|654738300|ref|WP_028194554.1| 34.51

142

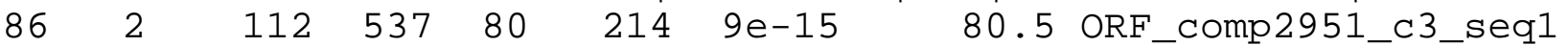

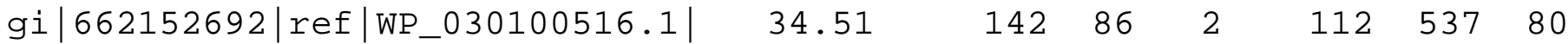

214 1e-14 80.5 ORF_comp2951_c3_seq1 gi|91691483|gb|ABE34681.1|

$\begin{array}{llllllllll}33.80 & 142 & 87 & 2 & 112 & 537 & 44 & 178 & 2 \mathrm{e}-14 & 79.0\end{array}$

ORF_comp2951_c3_seq1 gi|739030777|ref|WP_036911908.1| 36.11

144

$82 \quad 5 \quad 121 \quad 546 \quad 52 \quad 187 \quad 3 e-14 \quad 79.0 \quad$ ORF_comp2951_c3_seq1

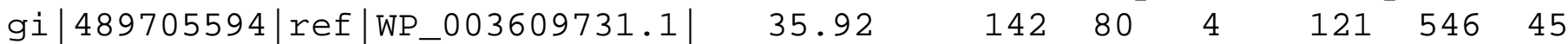

175 3e-14 78.6 ORF_comp2951_c3_seq1

gi $|749301086|$ ref |WP_040125311.1| 33.80

228 3e-14 79.0 ORF_comp2951_c3_seq1

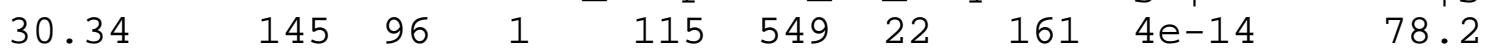

ORF_comp2951_c3_seq1 gi|207082025|gb|ABS78257.2| 30.34 14596

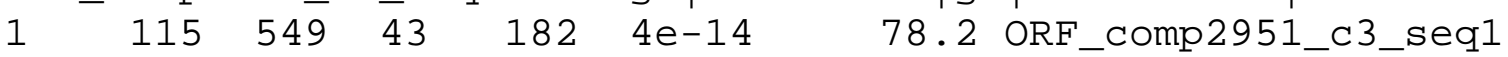

gi|165917203|gb|EDR35807.1| $30.34 \quad 145 \quad 96 \quad 1 \quad \begin{array}{lllll}115 & 549 & 81 & 220\end{array}$

4e-14 78.6 ORF_comp2951_c3_seq1 gi|754969497|ref|WP_042325399.1|

$\begin{array}{llllllllll}37.32 & 142 & 82 & 4 & 112 & 537 & 73 & 207 & 1 \mathrm{e}-13 & 77.4\end{array}$

ORF_comp2951_c3_seq1 gi|748629167|ref|WP_039887344.1| 33.33

$832 \quad 115 \quad 546 \quad 43 \quad 173 \quad 2 e-13 \quad 76.3$ ORF_comp2951_c3_seq1

gi|333458192|gb|EGK86811.1| $33.33 \quad 144 \quad 83 \quad 2 \quad 115 \quad 546 \quad 73 \quad 203$

2e-13 76.3 ORF_comp2951_c3_seq1 gi|800135199|dbj|GA034503.1|

$\begin{array}{llllllllll}35.34 & 133 & 79 & 4 & 148 & 546 & 44 & 169 & 3 e-13 & 75.9\end{array}$

ORF_comp2951_c3_seq1 gi|797211671|ref|WP_045872614.1| 34.72

144

$84 \quad 4 \quad 121546 \quad 46 \quad 181 \quad 3 e-13 \quad 75.5$ ORF_comp2951_c3_seq1

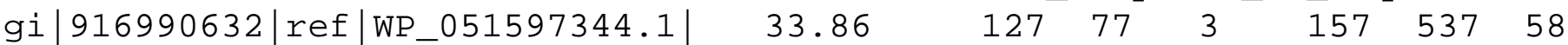

177 6e-13 75.1 ORF_comp2951_c3_seq1

gi|981354997|ref|WP_059570263.1| 34.51

174 2e-12 73.6 ORF_comp2951_c3_seq1

gi $|504939174|$ ref|WP_015126276.1| 31.47

187 2e-12 73.6 ORF_comp2951_c3_seq1

gi|966518160|ref|WP_058534654.1| 31.43

$1696 \mathrm{e}-11$ 68.9 ORF_comp2951_c3_seq1

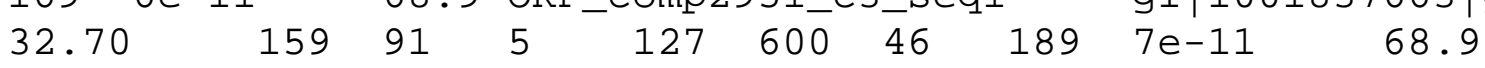

ORF_comp2951_c3_seq1 gi|500232799|ref|WP_011902392.1| 30.56

$\begin{array}{llllll}142 & 86 & 3 & 121 & 546 & 40\end{array}$

$\begin{array}{llllll}143 & 90 & 2 & 118 & 546 & 53\end{array}$

$\begin{array}{llllll}140 & 88 & 4 & 130 & 549 & 38\end{array}$

gi|1001837663|gb|KXS32259.1|

$\begin{array}{lllllll}91 & 4 & 121 & 549 & 38 & 173 & 1 \mathrm{e}-10\end{array}$

gi|931478713|gb|KPK59845.1| 35.21

3e-10 67.0 ORF_comp2951_c3_seq1

$\begin{array}{llllllllll}33.99 & 153 & 81 & 5 & 121 & 549 & 48 & 190 & 7 \mathrm{e}-10 & 66.2\end{array}$

ORF_comp2951_c3_seq1 $\quad$ gi|827519218|gb|KLN61905.1| 26.39 14499

$3 \quad 115 \quad 546 \quad 24 \quad 160 \quad 2 \mathrm{e}-09 \quad 64.3$ ORF_comp2951_c3_seq1

gi|518404331|ref|WP_019574538.1| 34.65

151 2e-09 64.7 ORF_comp2951_c3_seq1

gi|920578875|ref|WP_053005650.1| 26.39

176 3e-09 63.9 ORF_comp2951_c3_seq1

gi $\mid 497514158$ |ref |WP_009828356.1| 28.87

68.2 ORF_comp2951_c3_seq1

144

167 2e-08 62.0 ORF_comp2951_c3_seq1

$28.17 \quad \begin{array}{lllllll}142 & 95 & 3 & 121 & 546 & 34 & 168\end{array}$

ORF_comp2951_c3_seq1

gil496167609|ref/WP $2 \mathrm{e}-08$ - 61.6

$\begin{array}{llllll}101 & 61 & 1 & 115 & 417 & 56\end{array}$

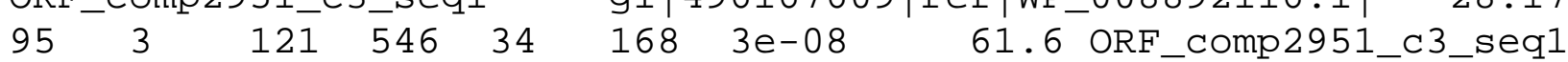

gi|817130062 | ref |WP_046502581.1| 25.69

$1764 \mathrm{e}-08$ 61.2 ORF_comp2951_c3_seq1

gi $\mid 522080484$ |ref |WP_020591693.1| 26.39

$\begin{array}{llllll}144 & 99 & 3 & 115 & 546 & 40\end{array}$

$\begin{array}{llllll}142 & 94 & 3 & 121 & 546 & 33\end{array}$

gi | 1000089089 | gb|KXJ56520.1 |

$2 \mathrm{e}-08 \quad 61.6$

$1724 \mathrm{e}-08 \quad 60.8$ ORF_comp2951_c3_seq1

$\begin{array}{llllll}144 & 100 & 3 & 115 & 546 & 40\end{array}$

$\begin{array}{llllll}144 & 99 & 3 & 115 & 546 & 36\end{array}$ 
gi|213409680|ref|XP_002175610.1| 30.34 190 9e-08 61.2 ORF_comp2951_c3_seq1 gi|966405784|ref|WP_058449190.1| 32.94 86 9e-08 59.3 ORF_comp2951_c3_seq1 gi|503689125|ref|WP_013923201.1| 36.11 138 1e-07 58.5 ORF_comp2951_c3_seq1 gi|490267384|ref|WP_004163951.1| 29.27 179 2e-07 59.3 ORF_comp2951_c3_seq1 gi |918692141|ref|WP_052561880.1| 30.00 164 2e-07 58.9 ORF_comp2951_c3_seq1 gi|490258075|ref|WP_004155296.1| 29.27 193 2e-07 59.3 ORF_comp2951_c3_seq1 gi|831778908|ref|XP_012755554.1| 25.38 2004 e-07 59.3 ORF_comp2951_c3_seq1

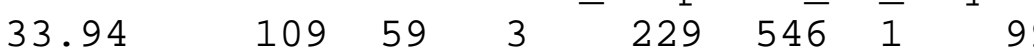
ORF_comp2951_c3_seq1 gi $|813208513| d b j|G A 050417.1| 29.32$ $\begin{array}{llllll}145 & 85 & 5 & 121 & 546 & 59\end{array}$ $\begin{array}{llllll}85 & 49 & 2 & 157 & 408 & 9\end{array}$ $\begin{array}{llllll}108 & 58 & 5 & 121 & 429 & 37\end{array}$ $\begin{array}{llllll}164 & 93 & 5 & 121 & 546 & 17\end{array}$ $\begin{array}{llllll}140 & 83 & 3 & 130 & 549 & 40\end{array}$ $\begin{array}{llllll}164 & 93 & 5 & 121 & 546 & 31\end{array}$ $\begin{array}{llllll}130 & 82 & 4 & 160 & 546 & 85\end{array}$ gi $\mid 925230922$ |gb|K0S75550.1| $4 \mathrm{e}-07 \quad 57.0$

$4 \quad 154546 \quad 1462635 \mathrm{e}-07 \quad 59.3$ ORF_comp2951_c3_seq1 gi|310766388|gb|ADP11338.1| $29.27 \quad 164 \quad 93 \quad 5 \quad \begin{array}{lllll}121 & 546 & 44 & 206\end{array}$ 5e-07 58.5 ORF_comp2951_c3_seq1 gi|752845383|ref|WP_041474394.1| $\begin{array}{llllllllll}29.27 & 164 & 93 & 5 & 121 & 546 & 31 & 193 & 5 e-07 & 58.2\end{array}$ ORF_comp2951_c3_seq1 gi|752845037|ref|WP_041474048.1| 29.27

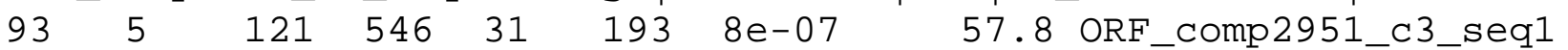
gi|224965368|emb|CAX56900.1| $29.27 \quad 164 \quad 93 \quad 5 \quad 121 \quad 546 \quad 44 \quad 206$ 9e-07 57.8 ORF_comp2951_c3_seq1 gi|915474789|ref|WP_050814322.1| $\begin{array}{llllllllll}30.84 & 107 & 65 & 3 & 229 & 546 & 1 & 99 & 1 \mathrm{e}-06 & 55.8\end{array}$ ORF_comp2951_c3_seq1 gi|872579356|ref|WP_048533013.1| 27.91 $84 \quad 4 \quad 157 \quad 537 \quad 144 \quad 265 \quad 1 \mathrm{e}-06 \quad 57.8$ ORF_comp2951_c3_seq1 gi|497652379|ref|WP_-009966563.1| $30.28 \quad 142 \quad 69 \quad 3 \quad 121 \quad 546 \quad 40$ 151 2e-06 56.2 ORF_comp2951_c3_seq1 $\begin{array}{llllllllll}30.28 & 142 & 69 & 3 & 121 & 546 & 30 & 141 & 2 \mathrm{e}-06 & 55.8\end{array}$ ORF_comp2951_c3_seq1 gi|736937357|ref|WP_034934372.1| 28.05 $95 \quad 5 \quad 121 \quad 546 \quad 32 \quad 194$ 3e-06 56.2 ORF_comp2951_c3_seq1 gi|517208875 |ref|WP_018397693.1| 38.38 134 3e-06 54.7 ORF_comp2951_c3_seq1 gi|923123265|ref|XP_013755101.1| 28.79 158 3e-06 56.6 ORF_comp2951_c3_seq1 gi|308510244|ref|XP_003117305.1| 29.01 166 3e-06 56.6 ORF_comp2951_c3_seq1 gi|737401579|ref|WP_035382755.1| 32.18 125 4e-06 55.5 ORF_comp2951_c3_seq1 gi|493073505|ref|WP_006122095.1| 27.11 194 4e-06 55.8 ORF_comp2951_c3_seq1

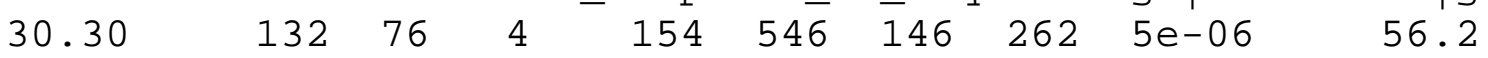
ORF_comp2951_c3_seq1 gi|857976683|emb|CE097478.1| 26.72 $3 \quad 160546 \quad 39 \quad 156 \quad 8 \mathrm{e}-06 \quad 55.5$ ORF_comp2951_c3_seq1 gi|953489559|emb|CEG46925.1| $30.23 \quad 129 \quad 78 \quad 4 \quad \begin{array}{lllll}160 & 546 & 188 & 304\end{array}$ 9e-06 55.5 ORF_comp2951_c3_seq1

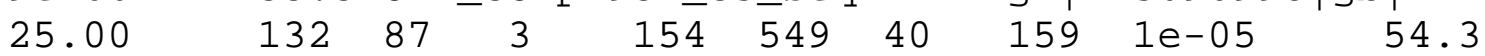
ORF_comp2951_c3_seq1 gi|800137352|dbj|GA033130.1| 29.13 $\begin{array}{llllll}132 & 79 & 5 & 160 & 546 & 39\end{array}$ $\begin{array}{llllll}131 & 78 & 5 & 160 & 546 & 49\end{array}$ $\begin{array}{llllll}87 & 54 & 1 & 157 & 417 & 44\end{array}$ $\begin{array}{llllll}166 & 98 & 5 & 115 & 546 & 30\end{array}$ gi $\mid 672825823$ | gb|KFH70714 . $1 \mid$ 13181 $2 \quad 157450 \quad 46 \quad 143 \quad 1 \mathrm{e}-05 \quad 54.3$ ORF_comp2951_c3_seq1 gi|159485978|ref|XP_001701021.1| 27.69

134 1e-05 53.1 ORF_comp2951_c3_seq1 $28.46 \quad 130 \quad 80 \quad 3$
ORF_comp2951_c3_seq1 $\begin{array}{lllll}160 & 546 & 41 & 158 & 2 \mathrm{e}-05\end{array}$ $\begin{array}{llllll}130 & 81 & 3 & 160 & 546 & 17\end{array}$ gi $\mid 341888907$ |gb|EGT44842 .1 | ORF_comp2951_c3_seq1 gi|502816264|ref|WP_013051240.1| 28.14

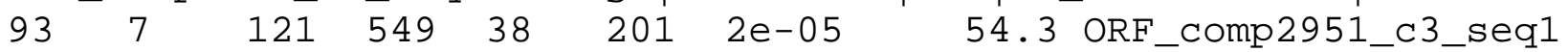
gi|290993528|ref|XP_002679385.1| 32.06 172 2e-05 53.5 ORF_comp2951_c3_seq1 $\begin{array}{llllll}131 & 75 & 5 & 154 & 546 & 56\end{array}$ gi $|309365040|$ emb | CAP23744 . 2 | 

$\begin{array}{lll}131 & 77 \quad 5\end{array}$

$160 \quad 546 \quad 41 \quad 158 \quad 3 e-05$

53.9

ORF_comp2951_c3_seq1

gi|301112447|ref|XP_002997994.1| 29.46

$79 \quad 4 \quad 160 \quad 546 \quad 227 \quad 343 \quad 3 e-05 \quad 53.9$ ORF_comp2951_c3_seq1

gi|301112441|ref|XP_002997991.1| 28.68

$\begin{array}{llllll}129 & 80 & 4 & 160 & 546 & 80\end{array}$

196 3e-05 53.5 ORF_comp2951_c3_seq1

gi $\mid 922867416$ | gb |K0034056 . 1 |

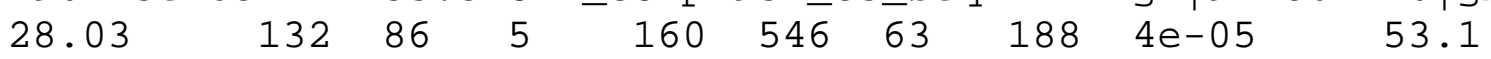

ORF_comp2951_c3_seq1 gi|302847090|ref|XP_002955080.1| 29.55

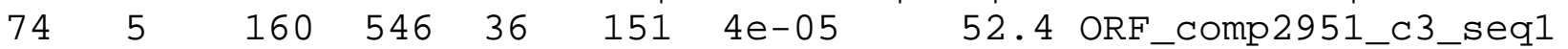

gi|520899875|ref|WP_020322885.1| $26.83 \quad 164 \quad 97 \quad 5 \quad \begin{array}{lllll}121 & 546 & 32\end{array}$

194 4e-05 53.1 ORF_comp2951_c3_seq1

gi|268531908|ref|XP_002631082.1| 29.77

158 4e-05 53.1 ORF_comp2951_c3_seq1

$28.68 \quad 129 \quad 80 \quad 4$
ORF comp2951_c3 seq1

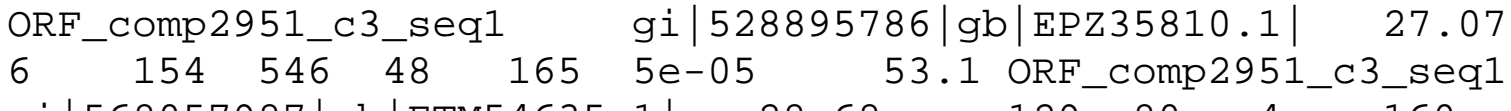

$\begin{array}{llllll}131 & 77 & 5 & 160 & 546 & 41\end{array}$ gi $\mid 570996289$ | gb |ETP52930.1|

5e- $05 \quad 53.1$

gi|568057987|gb|ETM54635.1| 28.68

5e-05 53.1 ORF_comp2951_c3_seq1

28.68

$12980 \quad 4$

$160 \quad 546 \quad 222$

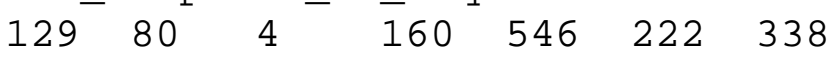

gi $\mid 970660098$ |gb|KUF97679.1|

ORF_comp2951_c3_seq1 gi|675213985|ref|XP_008915877.1| 28.68

129

$80 \quad 4 \quad 160 \quad 546222338$ 5e-05 53.1 ORF_comp2951_c3_seq1

gi|544211776|ref|XP_005536287.1| 29.66

277 5e-05 53.1 ORF_comp2951_c3_seq1

gi|695439924|ref|XP_009532833.1| 29.46

356 6e-05 52.8 ORF_comp2951_c3_seq1

gi|193204637|ref|NP_001122616.1| 29.01

158 6e-05 52.8 0RF_comp2951_c3_seq1

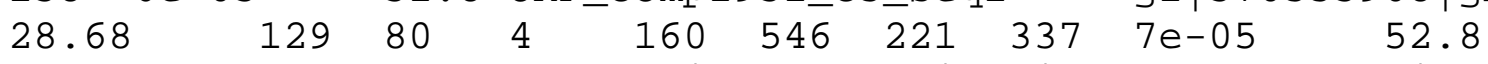
ORF_comp2951_c3_seq1 gi|193204635|ref|NP_001122615.1| 29.01

$\begin{array}{llllll}129 & 79 & 4 & 160 & 546 & 240\end{array}$

$\begin{array}{llllll}131 & 78 & 5 & 160 & 546 & 41\end{array}$ gi $|570335908| \mathrm{gb} \mid \mathrm{ET083856.1|}$

$\begin{array}{lllllll}78 & 5 & 160 & 546 & 49 & 166 & 7 \mathrm{e}-05\end{array}$

52.8 ORF_comp2951_c3_seq1

$\begin{array}{llr}\text { gi|} 14485518|g b| & \text { AAK62991.1|AF299332_1 } 29.01 \\ 41 \quad 1589 e-05 \quad 52.4 \text { ORF_comp2951_c3_seq1 }\end{array}$

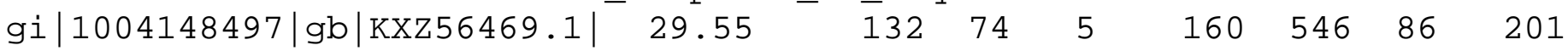

1e-04 52.4 ORF_comp2951_c3_seq1 gi|505014688|ref|WP_015201790.1|

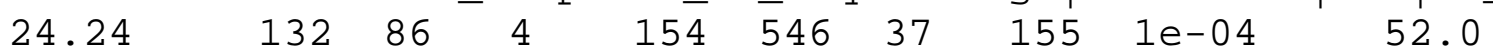

ORF_comp2951_c3_seq1 gi|303281270|ref|XP_003059927.1| 33.33

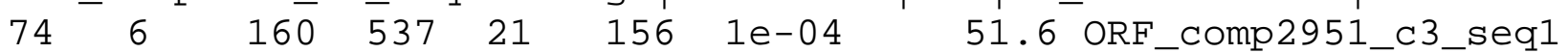

gi|970651951|gb|KUF90699.1| $27.91 \quad 0129 \quad 81 \quad 4 \quad \begin{array}{lllll}160 & 546 & 222 & 338\end{array}$

1e-04 52.0 ORF_comp2951_c3_seq1 gi|570959955|gb|ETP24934.1|

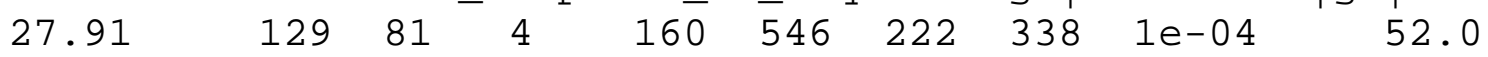

ORF_comp2951_c3_seq1 gi|499786267|ref|WP_011467001.1| 24.36

$97 \quad 3 \quad 121 \quad 531 \quad 93 \quad 246 \quad 2 \mathrm{e}-04 \quad 51.6$ ORF_comp2951_c3_seq1

gi|567998271|gb|ETL48346.1| $27.91 \quad 129 \quad 81 \quad 4 \quad 160 \quad 546 \quad 222 \quad 338$

2e-04 51.6 ORF_comp2951_c3_seq1

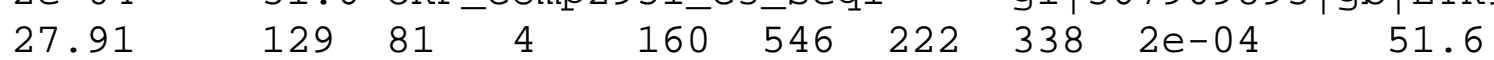

ORF_comp2951_c3_seq1 gi|504984674|ref|WP_015171776.1| 25.00

$85 \quad 4 \quad 154 \quad 546 \quad 39 \quad 157 \quad 3 e-04 \quad 51.2$ ORF_comp2951_c3_seq1

gi|748749862|ref|WP_-040007977.1| 31.18

$1404 \mathrm{e}-04 \quad 50.4$ ORF_comp2951_c3_seq1

gi|599364119|ref|XP_007405638.1| 27.74

$\begin{array}{lllll}93 & 57 & 3 & 160 & 432\end{array}$

131

$186 \quad 5 e-04 \quad 49.7$

$\begin{array}{llllll}137 & 79 & 5 & 160 & 546 & 62\end{array}$

ORF_comp2951_c3_seq2

gi|501733845|ref|WP_012629148.1| 42.44

205

$1084 \quad 115 \quad 72344 \quad 240 \quad 5 \mathrm{e}-40 \quad 153$ ORF_comp2951_c3_seq2

gi|516956783|ref|WP_018183684.1| $41.06 \quad 20 \overline{7} 113 \quad 4 \quad 121 \quad 735 \quad 39$

238 2e-39 151 ORF_comp2951_c3_seq2 gi|740234212|ref|WP_038075400.1|

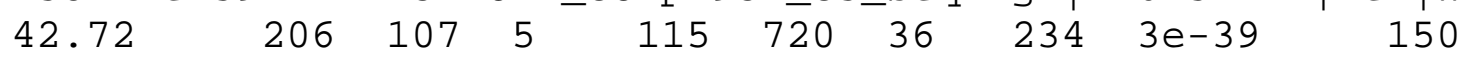


ORF_comp2951_c3_seq2

gi|493029431|ref|WP_006099075.1| 40.29

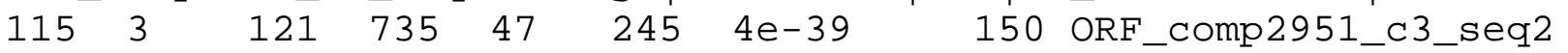

gi|751277775|ref|WP_040986624.1| $40.98 \quad 20 \overline{5} \quad 112 \quad 4 \quad \begin{array}{lllll}115 & 723 & 36\end{array}$

$2335 e-39 \quad 150$ ORF_comp2951_c3_seq2 gi|751301901|ref|WP_041010414.1|

$\begin{array}{llllllllll}40.98 & 205 & 112 & 4 & 115 & 723 & 36 & 233 & 5 e-39 & 150\end{array}$

ORF_comp2951_c3_seq2 gi|751301199|ref|WP_041009719.1| 40.98

205

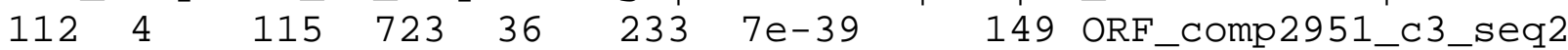

gi|501377640|ref|WP_012409206.1| $39.81 \quad 206 \quad 115 \quad 5 \quad 115 \quad 726 \quad 41$

239 8e-39 149 ORF_comp2951_c3_seq2 gi|522054471|ref|WP_020565680.1|

$\begin{array}{llllllllll}38.91 & 221 & 111 & 5 & 121 & 762 & 54 & 257 & 2 \mathrm{e}-38 & 149\end{array}$

ORF_comp2951_c3_seq2 gi|515385832|ref|WP_016877723.1| 41.55

207

$106 \quad 6 \quad 121 \quad 726 \quad 46 \quad 242$ 3e-38 148 ORF_comp2951_c3_seq2

gi|505020123|ref|WP_015207225.1| $41.06 \quad 207 \quad 111 \quad 5 \quad 115 \quad 726 \quad 44$

242 3e-38 148 ORF_comp2951_c3_seq2 gi|763312982|ref|WP_044171432.1|

$\begin{array}{llllllllll}40.29 & 206 & 115 & 3 & 121 & 735 & 38 & 236 & 7 \mathrm{e}-38 & 147\end{array}$

ORF_comp2951_c3_seq2 gi|499931312|ref|WP_011612046.1| 41.46

205

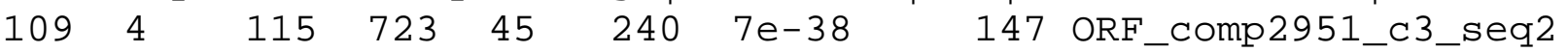

gi|756829253|ref|WP_042646352.1| $40.49 \quad 20 \overline{5} 113 \quad 4 \quad \begin{array}{lllll}115 & 723 & 36\end{array}$

$2331 \mathrm{e}-37 \quad 146$ ORF_comp2951_c3_seq2 gi|751264288|ref|WP_040974001.1|

$\begin{array}{llllllllll}40.00 & 205 & 114 & 4 & 115 & 723 & 38 & 235 & 1 \mathrm{e}-37 & 146\end{array}$

ORF_comp2951_c3_seq2 gi|685089209|emb|CDX24205.1| 40.00 $205 \quad 114$

$4 \quad \begin{array}{llllll}115 & 723 & 36 & 233 & 1 \mathrm{e}-37 & 146 \text { ORF_comp2951_c3_seq2 }\end{array}$

gi|689678301|emb|CEF48871.1| $40.78 \quad 206 \quad 107 \quad 4 \quad \begin{array}{lllll}121 & 723 & 40 & 235\end{array}$

1e-37 146 ORF_comp2951_c3_seq2 gi|503088795|ref|WP_013323642.1|

$\begin{array}{llllllllll}40.69 & 204 & 112 & 4 & 121 & 726 & 47 & 243 & 2 \mathrm{e}-37 & 146\end{array}$

ORF_comp2951_c3_seq2 gi|981461217|ref|WP_059669889.1| 41.75

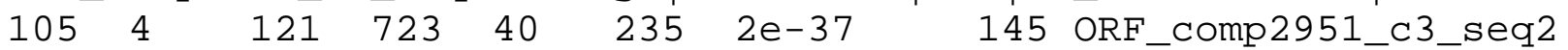

gi|504925739|ref|WP_015112841.1| $40.20 \quad 204 \quad 113 \quad 4 \quad 121 \quad 726 \quad 46$

242 3e-37 145 ORF_comp2951_c3_seq2 gi|927917924|emb|CDX53272.1|

$\begin{array}{llllllllll}40.00 & 205 & 114 & 4 & 115 & 723 & 36 & 233 & 3 e-37 & 145\end{array}$

ORF_comp2951_c3_seq2 gi|826018244|ref|WP_047165229.1| 41.46

$1094 \quad 115 \quad 723 \quad 33 \quad 2284 \mathrm{e}-37 \quad 145$ ORF_comp2951_c3_seq2

gi|656719681|gb|KEF42411.1| $43.00 \quad 207 \quad 10 \overline{5} 6 \quad 6 \quad 112 \quad 720 \quad 22$

6e-37 144 ORF_comp2951_c3_seq2 gi|748165414|ref|WP_039738990.1|

$\begin{array}{llllllllll}39.90 & 203 & 113 & 5 & 121 & 723 & 46 & 241 & 6 e-37 & 144\end{array}$

ORF_comp2951_c3_seq2 gi|814343774|emb|CPH68122.1| $42.23 \quad 206 \quad 104$

$4 \quad \begin{array}{llllll}121 & 723 & 38 & 233 & 7 \mathrm{e}-37 & 144 \text { ORF_comp2951_c3_seq2 }\end{array}$

gi|648291774|ref|WP_026072923.1| $40.29 \quad-206 \quad 114 \quad 3 \quad 121 \quad 73546$

243 7e-37 144 ORF_comp2951_c3_seq2 gi|648412694|ref|WP_026104445.1|

$\begin{array}{llllllllll}38.65 & 207 & 119 & 4 & 121 & 735 & 46 & 246 & 8 e-37 & 144\end{array}$

ORF_comp2951_c3_seq2 gi|505018197|ref|WP_015205299.1| 41.18

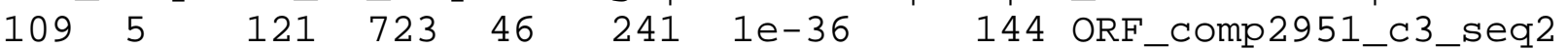

gi|484585464|gb|AGK47089.1| $41.26 \quad 206 \quad 106 \quad 4 \quad 121 \quad 723 \quad 30$

204

2e-36 143 ORF_comp2951_c3_seq2 gi|499442921|ref|WP_011130385.1|

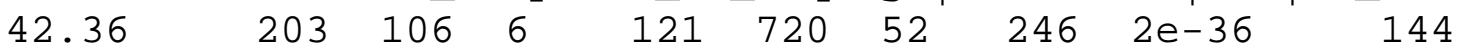

ORF_comp2951_c3_seq2

gi|754360430|ref|WP_041861630.1| 41.26

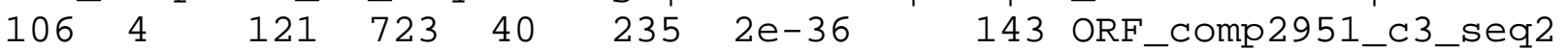

gi|773064690|gb|AJY42591.1| $41.26 \quad 206 \quad 10 \overline{6} \quad 4 \quad 1 \overline{1} 1$ - $723 \quad 30$

225

2e-36 142 ORF_comp2951_c3_seq2 gi|981741072|ref|WP_059934588.1|

$\begin{array}{llllllllll}41.26 & 206 & 106 & 4 & 121 & 723 & 40 & 235 & 2 e-36 & 143\end{array}$

ORF_comp2951_c3_seq2 gi|737172391|ref|WP_035158595.1| 39.32

$1125 \quad 121 \quad 726 \quad 43 \quad 239$ 3e-36 143 ORF_comp2951_c3_seq2

gi|738534441|ref|WP_036482850.1| $37.44 \quad 203 \quad 123 \quad 3 \quad 118 \quad 720 \quad 46$

246 3e-36 143 ORF_comp2951_c3_seq2 gi|492895672|ref|WP_006026078.1|

$\begin{array}{llllllllll}41.26 & 206 & 106 & 4 & 121 & 723 & 40 & 235 & 3 e-36 & 142\end{array}$

ORF_comp2951_c3_seq2 gi|913438795|ref|WP_050418395.1| 40.39

203

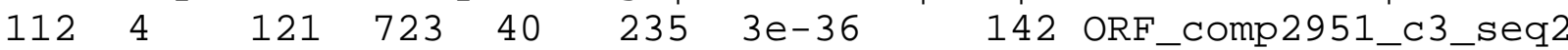


gi $\mid 501395618$ |ref |WP_012427184.1|

42.11

$209 \quad 113 \quad 4$

112735

81

$2823 e-36$

144 ORF_comp2951

3 seq2 gi

gi| 909830227 |gb|AKU13025.1|

$\begin{array}{llllllllll}40.39 & 203 & 112 & 4 & 121 & 723 & 45 & 240 & 3 e-36 & 142\end{array}$

ORF_comp2951_c3_seq2

gi|584107904|gb|AHI79522.1| 41.67

204108

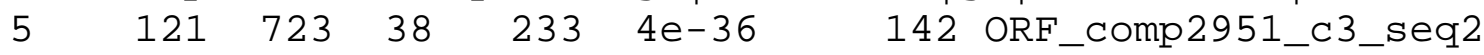

gi|505013627|ref|WP_015200729.1| $37.98 \quad 208 \quad 120 \quad 4 \quad 118 \quad 735 \quad 45$

245 4e-36 142 ORF_comp2951_c3_seq2 gi|974600092|ref|WP_059190108.1|

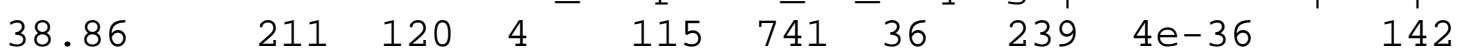

ORF_comp2951_c3_seq2 gi|740984489|ref|WP_038768164.1| 41.75

206

$1054 \quad 121 \quad 72340 \quad 2354 \mathrm{e}-36 \quad 142$ ORF_comp2951_c3_seq2

gi|497576287|ref|WP_009890471.1| $41.67 \quad 204 \quad 108 \quad 5 \quad \begin{array}{lllll}121 & 723 & 40\end{array}$

235 4e-36 142 ORF_comp2951_c3_seq2 gi|657935844|ref|WP_029636980.1|

$\begin{array}{llllllllll}39.41 & 203 & 114 & 5 & 121 & 723 & 46 & 241 & 4 \mathrm{e}-36 & 142\end{array}$

ORF_comp2951_c3_seq2 gi|497590677|ref|WP_009904861.1| 41.38

$1104 \quad 121 \quad 72340 \quad 235$ 5e-36 142 ORF_comp2951_c3_seq2

gi|782673738|ref|WP_045600604.1| $41.38 \quad 20 \begin{array}{llllll}110 & 4 & 121 & 723 & 40\end{array}$

235 5e-36 142 ORF_comp2951_c3_seq2 gi|493165631|ref|WP_006170876.1|

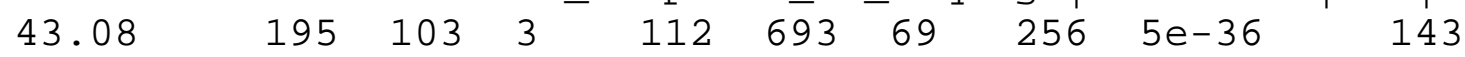

ORF_comp2951_c3_seq2 gi|91691483|gb|ABE34681.1| 40.67 $209 \quad 116$

$3 \quad 112735 \quad 44 \quad 245 \quad 6 e-36 \quad 142$ ORF_comp2951_c3_seq2

gi|981383968|ref|WP_059597148.1| $41.75 \quad 20 \begin{array}{llllll}206 & 105 & 4 & 121 & 723 & 30\end{array}$

225 6e-36 142 ORF_comp2951_c3_seq2 gi|953510847|dbj|BAT53535.1|

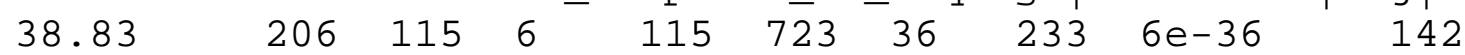

ORF_comp2951_c3_seq2 gi|515356232|ref|WP_016865554.1| 39.32

$1165 \quad 115 \quad 726 \quad 43 \quad 241 \quad 7 e-36 \quad 142$ ORF_comp2951_c3_seq2

gi|981645748|ref|WP_059844321.1| $41.67 \quad 204 \quad 108 \quad 5 \quad 121 \quad 723 \quad 40$

235 7e-36 142 ORF_comp2951_c3_seq2 gi|821561551|ref|WP_046868556.1|

$\begin{array}{llllllllll}41.38 & 203 & 110 & 4 & 121 & 723 & 41 & 236 & 8 \mathrm{e}-36 & 141\end{array}$

ORF_comp2951_c3_seq2 gi|652338179|ref|WP_026735392.1| 40.38

$1116 \quad 115 \quad 726 \quad 44 \quad 242 \quad 1 \mathrm{e}-35 \quad 141$ ORF_comp2951_c3_seq2

gi|749301086|ref|WP_040125311.1| $40.67 \quad 209 \quad 116 \quad 3 \quad r \begin{array}{llll}112 & 735 & 94\end{array}$

$2951 e-35 \quad 142$ ORF_comp2951_c3_seq2 gi|976471317|gb|KVD74754.1|

$\begin{array}{llllllllll}40.78 & 206 & 107 & 4 & 121 & 723 & 30 & 225 & 2 \mathrm{e}-35 & 140\end{array}$

ORF_comp2951_c3_seq2 gi|917776332|ref|WP_052290273.1| 39.81

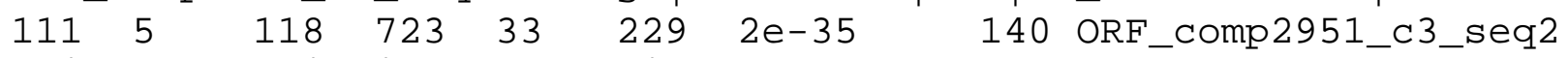

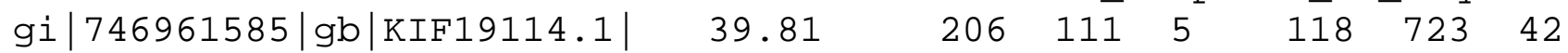

2e-35 140 ORF_comp2951_c3_seq2 gi|941312503|ref|WP_055116231.1|

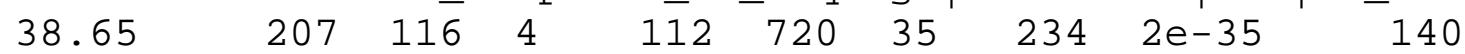

ORF_comp2951_c3_seq2 gi|981296166|ref|WP_059513522.1| 40.89

$111^{4} 121 \quad 723 \quad 39234 \quad 2 \mathrm{e}-35 \quad 140$ ORF_comp2951_c3_seq2

gi|740959588|ref|WP_038744210.1| $41.26 \quad 206 \quad 106 \quad 4 \quad \begin{array}{lllll}121 & 723 & 40\end{array}$

235 2e-35 140 ORF_comp2951_c3_seq2 gi|714646342|gb|KGW52040.1|

$\begin{array}{llllllllll}41.26 & 206 & 106 & 4 & 121 & 723 & 30 & 225 & 3 e-35 & 140\end{array}$

ORF_comp2951_c3_seq2 gi|656037395|ref|WP_029076335.1| 38.46

$1194 \quad 121 \quad 738 \quad 39 \quad 239 \quad 3 e-35 \quad 140$ ORF_comp2951_c3_seq2

$\begin{array}{llllllll}\text { gi|976553572|gb|KVE52227.1| } & 40.78 & 206 & 10 \overline{7} & 4 & 121 & 723 & 30\end{array}$

3e-35 140 ORF_comp2951_c3_seq2 gi|504949901|ref|WP_015137003.1|

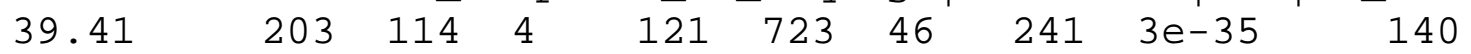

ORF_comp2951_c3_seq2 gi|494317175|ref|WP_007177410.1| 41.95

$1114112 \quad 72390 \quad 287$ 3e-35 141 ORF_comp2951_c3_seq2

gi|752524486|ref|WP_041196068.1| $41.26 \quad 206 \quad 106 \quad 4 \quad r \begin{array}{lllll}121 & 723 & 40\end{array}$

235 3e-35 140 ORF_comp2951_c3_seq2 gi|913356948|ref|WP_050376444.1|

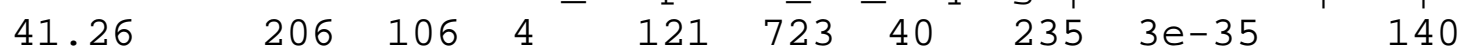

ORF_comp2951_c3_seq2 gi|800785064|emb|CFL25926.1| $41.26 \quad 206 \quad 106$

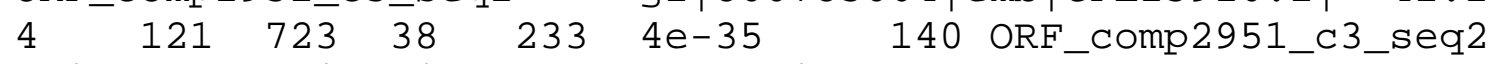

gi|953804284|ref|WP_058039816.1| $41.26 \quad-206 \quad 106 \quad 4 \quad 121 \quad 72340$

235 4e-35 140 ORF_comp2951_c3_seq2 gi|490685647|ref|WP_004550267.1| 
$41.26 \quad 2061064$
ORF_comp2951_c3_seq2

$\begin{array}{llllll}121 & 723 & 38 & 233 & 4 \mathrm{e}-35 & 140\end{array}$

gi|714507186|gb|KGV37859.1| 41.26

206106

$4 \quad 121723 \quad 30 \quad 225 \quad 4 \mathrm{e}-35 \quad$ 139 ORF_comp2951_c3_seq2

gi|490301683|ref|WP_004197081.1| $41.26 \quad-206 \quad 106 \quad 4 \quad 121 \quad 723 \quad 40$

235 4e-35 140 ORF_comp2951_c3_seq2 gi|953803876|ref|WP_058039691.1|

$\begin{array}{llllllllll}41.26 & 206 & 106 & 4 & 121 & 723 & 40 & 235 & 4 \mathrm{e}-35 & 140\end{array}$

ORF_comp2951_c3_seq2 gi|490304232|ref|WP_004199532.1| 41.26

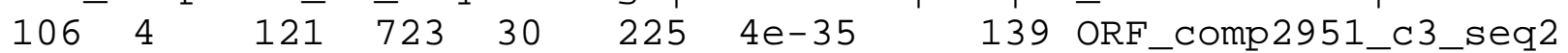

gi|981251097|ref|WP_059470554.1| $41.26 \quad 20 \overline{6} \quad 106 \quad 4 \quad \begin{array}{llll}121 & 723 & 39\end{array}$

234 4e-35 139 ORF_comp2951_c3_seq2 gi|737933697|ref|WP_035898371.1|

$\begin{array}{llllllllll}38.65 & 207 & 116 & 4 & 112 & 720 & 35 & 234 & 4 \mathrm{e}-35 & 139\end{array}$

ORF_comp2951_c3_seq2 gi|504970487|ref|WP_015157589.1| 39.71

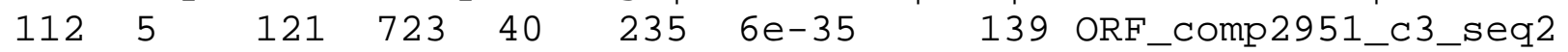

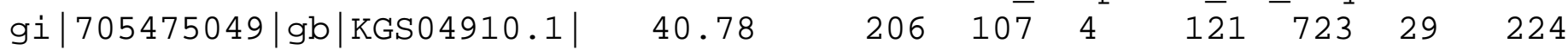

9e-35 138 ORF_comp2951_c3_seq2 gi|75702893|gb|ABA22569.1| 38.05

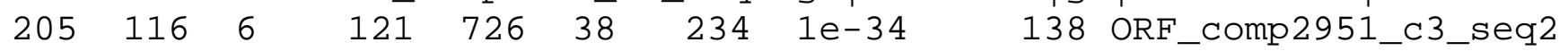

gi|981433289|ref|WP_059643234.1| $40.78 \quad 206 \quad 107 \quad 4 \quad 121 \quad 723 \quad 40$

235 1e-34 139 ORF_comp2951_c3_seq2 gi|740960548|ref|WP_038745121.1|

$\begin{array}{llllllllll}40.78 & 206 & 107 & 4 & 121 & 723 & 40 & 235 & 1 \mathrm{e}-34 & 138\end{array}$

ORF_comp2951_c3_seq2 gi|504945810|ref|WP_015132912.1| 39.13

207

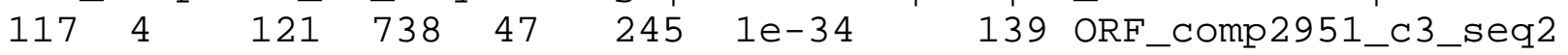

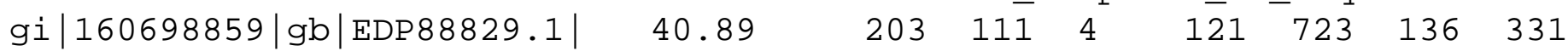
1e-34 140 ORF_comp2951_c3_seq2 gi|148027253|gb|EDK85274.1| 40.89

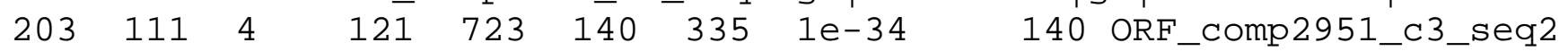

gi|243063500|gb|EES45686.1| $40.89 \quad 203 \quad 111 \quad 4 \quad \begin{array}{llllll}121 & 723 & 140 & 335\end{array}$

1e-34 140 ORF_comp2951_c3_seq2 gi|785594494|ref|WP_045719475.1|

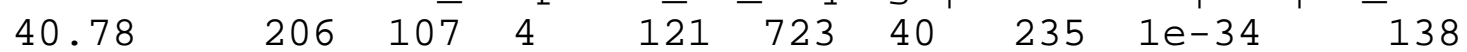

ORF_comp2951_c3_seq2 gi|126229014|gb|ABN92554.1| $40.89 \quad 203 \quad 111$

$4 \quad \begin{array}{llllll}121 & 723 & 145 & 340 & 1 \mathrm{e}-34 & 141 \text { ORF_comp2951_c3_seq2 }\end{array}$

gi|121227363|gb|ABM49881.1| $40.89 \quad 203 \quad 111 \quad 4 \quad r \begin{array}{lllll}121 & 723 & 142 & 337\end{array}$

1e-34 140 ORF_comp2951_c3_seq2 gi|741007236|ref|WP_038789710.1|

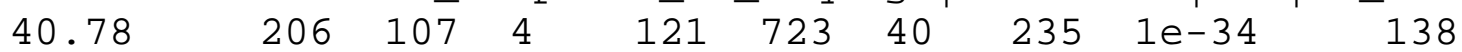

ORF_comp2951_c3_seq2 gi|157806771|gb|ED083941.1| 40.89 $203 \quad 111$

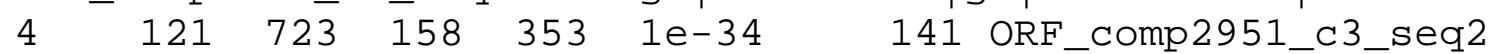

gi|169654349|gb|EDS87042.1| $40.89 \quad 203 \quad 111 \quad 4 \quad 121 \quad 723 \quad 155 \quad 350$ 1e-34 141 ORF_comp2951_c3_seq2 gi|806494169|gb|KKC16091.1| 40.78

$\begin{array}{llllllll}206 & 107 & 4 & 121 & 723 & 30 & 225 & 1 \mathrm{e}-34\end{array}$ 138 ORF_comp2951_c3_seq2

gi|740943421|ref|WP_038728427.1| $40.78 \quad 206 \quad 107 \quad 4 \quad 121 \quad 723 \quad 40$

235 1e-34 138 ORF_comp2951_c3_seq2 gi|237502694|gb|ACQ95012.1|

$\begin{array}{llllllllll}40.89 & 203 & 111 & 4 & 121 & 723 & 144 & 339 & 2 \mathrm{e}-34 & 140\end{array}$

ORF_comp2951_c3_seq2 gi|126219717|gb|ABN83223.1| $40.89 \quad 203 \quad 111$

$4 \quad \begin{array}{llllll}121 & 723 & 149 & 344 & 2 \mathrm{e}-34 & 140 \text { ORF_comp2951_c3_seq2 }\end{array}$

gi|652914138|ref|WP_027168079.1| $38.54 \quad 205 \quad 117 \quad 4 \quad 115 \quad 723 \quad 36$

233 2e-34 138 ORF_comp2951_c3_seq2 gi|157936951|gb|ED092621.1|

$\begin{array}{llllllllll}40.89 & 203 & 111 & 4 & 121 & 723 & 160 & 355 & 2 \mathrm{e}-34 & 141\end{array}$

ORF_comp2951_c3_seq2

gi|184209881|gb|EDU06924.1| 40.89

203111

$4 \quad 121723 \quad 151346 \quad 2 \mathrm{e}-34 \quad 140$ ORF_comp2951_c3_seq2

gi|254218068|gb|EETO7452.1| $40.89 \quad 203 \quad 111 \quad 4 \quad 121 \quad 723 \quad 162 \quad 357$

2e-34 141 ORF_comp2951_c3_seq2 gi|568795567|gb|AHE26011.1| 40.49

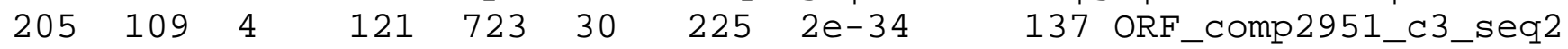

gi|217395731|gb|EEC35749.1| $40.89 \quad 203 \quad 111 \quad 4 \quad 121 \quad 723 \quad 168 \quad 363$

2e-34 141 ORF_comp2951_c3_seq2 gi|504891436|ref|WP_015078538.1|

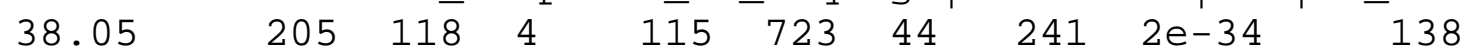

ORF_comp2951_c3_seq2 gi|714902087|gb|KGX30928.1| $40.89 \quad 203 \quad 111$

$4 \quad \begin{array}{llllll}121 & 723 & 152 & 347 & 2 \mathrm{e}-34 & 140 \text { ORF_comp2951_c3_seq2 }\end{array}$

gi|225927831|gb|EEH23872.1| $40.89 \quad 203 \quad 111 \quad 4 \quad 121 \quad 723 \quad 171 \quad 366$

2e-34 141 ORF_comp2951_c3_seq2 gi|134251017|gb|EBA51096.1| 40.89 
$\begin{array}{llllllll}203 & 111 & 4 & 121 & 723 & 166 & 361 & 2 \mathrm{e}-34\end{array}$ 141 ORF_comp2951_c3_seq2 gi|515888082|ref|WP_017318665.1| 37.93 $\begin{array}{llllll}203 & 117 & 4 & 121 & 723 & 46\end{array}$ $2412 \mathrm{e}-34 \quad 138$ ORF_comp2951_c3_seq2 gi|705585042|gb|KGS47698.1| $\begin{array}{llllllllll}40.78 & 206 & 107 & 4 & 121 & 723 & 30 & 225 & 2 \mathrm{e}-34 & 137\end{array}$ ORF_comp2951_c3_seq2 gi|686840559|gb|KGC20015.1| 40.78 $4 \quad \begin{array}{llllll}121 & 723 & 30 & 225 & 2 \mathrm{e}-34 & 137 \text { ORF_comp2951_c3_seq2 }\end{array}$ gi|497606907|ref|WP_009921091.1| $40.78 \quad-206 \quad 107 \quad 4 \quad 121 \quad 723 \quad 40$ 235 2e-34 138 ORF_comp2951_c3_seq2 gi|741015566|ref|WP_038797782.1|

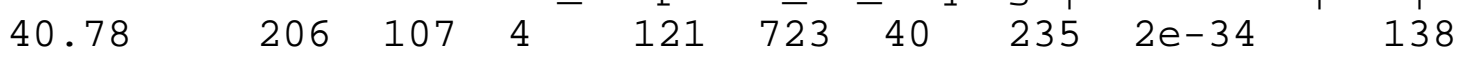
ORF_comp2951_c3_seq2 gi|17130321|dbj|BAB72932.1| 37.07 $205 \quad 118$ $6 \quad \begin{array}{llllll}121 & 726 & 41 & 237 & 2 \mathrm{e}-34 & 137 \text { ORF_comp2951_c3_seq2 }\end{array}$ gi|740986897|ref|WP_038770440.1| $40.49 \quad 205 \quad 109 \quad 4 \quad 121 \quad 723 \quad 40$ 235 2e-34 137 ORF_comp2951_c3_seq2 gi|764950513|ref|WP_044520790.1| $\begin{array}{llllllllll}37.07 & 205 & 118 & 6 & 121 & 726 & 38 & 234 & 2 e-34 & 137\end{array}$ ORF_comp2951_c3_seq2 gi|740942487|ref|WP_038727502.1| 40.78

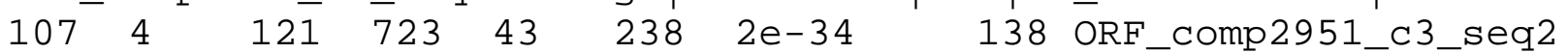

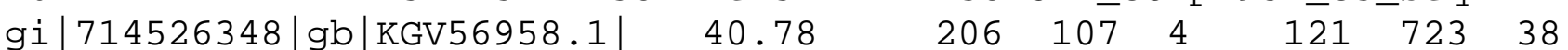
206

2e-34 137 ORF_comp2951_c3_seq2 gi|752526330|ref|WP_041197894.1|

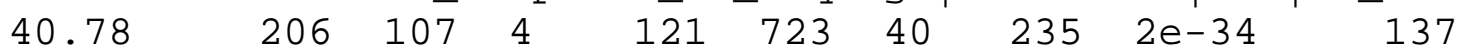
ORF_comp2951_c3_seq2 gi|714575209|gb|KGW05595.1| 40.78 $206 \quad 107$ $4 \quad \begin{array}{llllll}121 & 723 & 41 & 236 & 3 e-34 & 137 \text { ORF_comp2951_c3_seq2 }\end{array}$ gi|686848709|gb|KGC28119.1| $40.78 \quad 206 \quad 107 \quad 4 \quad \begin{array}{lllll}121 & 723 & 30 & 225\end{array}$ 3e-34 137 ORF_comp2951_c3_seq2 gi|740980945|ref|WP_038764816.1|

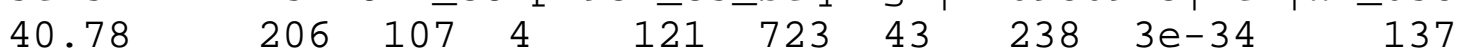

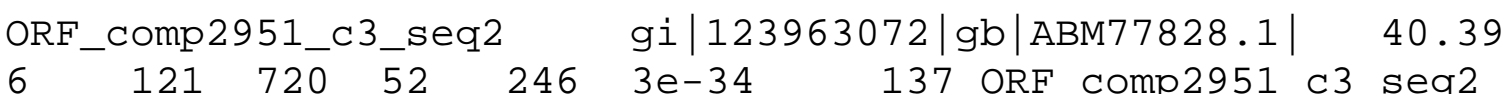

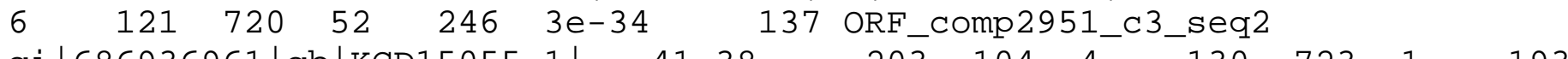

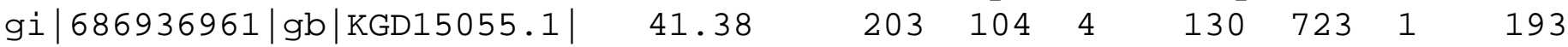
3e-34 136 ORF_comp2951_c3_seq2 gi|772972989|gb|AJX33047.1| 40.78

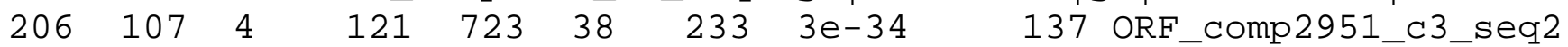
gi|685679385|gb|AI066505.1| $40.39 \quad 203 \quad 112 \quad 4 \quad 121 \quad 723 \quad 38$ 233 3e-34 137 ORF_comp2951_c3_seq2 gi|741019975|ref|WP_038801977.1|

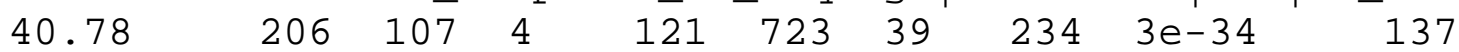
ORF_comp2951_c3_seq2 gi|741018909|ref|WP_038800916.1| 40.39

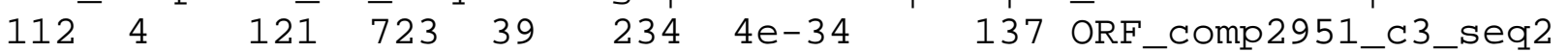
gi|495550750|ref|WP_008275329.1| $39.22 \quad 204 \quad 115 \quad 4 \quad 121 \quad 726 \quad 42$ 238 4e-34 137 ORF_comp2951_c3_seq2 gi|658419581|ref|WP_029646255.1|

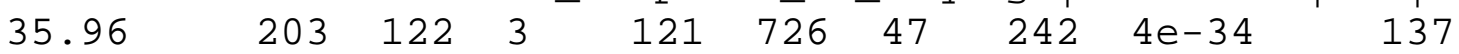
ORF_comp2951_c3_seq2 gi|916294818|ref|WP_051029864.1| 38.83 206 $115 \quad 5 \quad 115 \quad 723 \quad 42 \quad 239 \quad 4 \mathrm{e}-34 \quad 137$ ORF_comp2951_c3_seq2 gi|714591842|gb|KGW22095.1| $40.78 \quad 206 \quad 107 \quad 4 \quad 121 \quad 723 \quad 30$ 4e-34 137 ORF_comp2951_c3_seq2 gi|740967447|ref|WP_038751917.1| $\begin{array}{llllllllll}40.78 & 206 & 107 & 4 & 121 & 723 & 40 & 235 & 4 \mathrm{e}-34 & 137\end{array}$ ORF_comp2951_c3_seq2 gi|504967338|ref|WP_015154440.1| 39.02

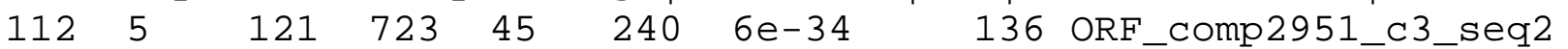
gi|754969497|ref|WP_042325399.1| $41.43 \quad 210 \quad 115 \quad 4 \quad 112 \quad 738$ 275 7e-34 137 ORF_comp2951_c3_seq2 gi|814476642|emb|CPI32267.1|

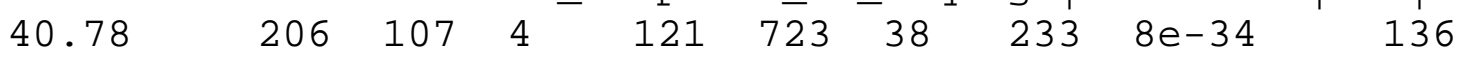
ORF_comp2951_c3_seq2 gi|497231444 |ref |WP_009545706.1| 38.92 225 $\begin{array}{lllllll}115 & 4 & 121 & 723 & 42 & 237 & 1 \mathrm{e}-33\end{array}$ 135 ORF_comp2951_c3_seq2 gi|496440563|ref|WP_009149408.1| $38.35 \quad 206 \quad 118 \quad 3 \quad \begin{array}{lllll}121 & 735 & 60\end{array}$ 257 2e-33 135 ORF_comp2951_c3_seq2 gi|751564302|ref|WP_041033204.1| $\begin{array}{llllllllll}37.44 & 203 & 118 & 4 & 121 & 723 & 46 & 241 & 3 e-33 & 135\end{array}$ ORF_comp2951_c3_seq2 gi|759580794|ref|WP_043299684.1| 40.19

$1056 \quad 121 \quad 723 \quad 40 \quad 243 \quad 9 \mathrm{e}-33 \quad 134$ ORF_comp2951_c3_seq2

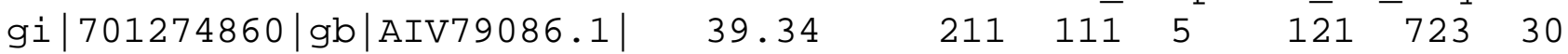

$9 e-33$

39.32 133 ORF_comp2951_c3_seq2 $206 \quad 108 \quad 4$

121723 gi | 640368848 | ref |WP_

$43 \quad 2369 e-33$

024880427.11 133 
ORF_comp2951_c3_seq2

gi|739030777|ref|WP_036911908.1| 40.39

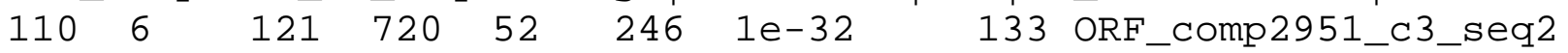

gi|648499655|ref|WP_026191406.1| $39.81 \quad 206 \quad 107 \quad 4 \quad \begin{array}{lllll}121 & 723 & 43\end{array}$

$2361 e-32133$ ORF_comp2951_c3_seq2 gi|966518160|ref|WP_058534654.1|

$\begin{array}{llllllllll}36.36 & 198 & 117 & 5 & 130 & 723 & 38 & 226 & 1 \mathrm{e}-32 & 132\end{array}$

ORF_comp2951_c3_seq2 gi|938273386|gb|KPQ๑8481.1| $39.11 \quad 202 \quad 115$

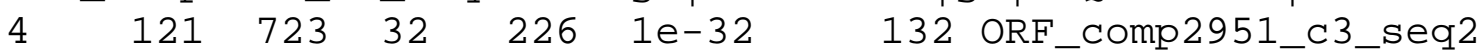

gi|85544315|pdb|2BTW|B $36.59 \quad 205 \quad 119$

32 133 ORF_comp2951_c3_seq2 gi|85544314|pdb|2BTW|A $36.59 \quad 205 \quad 119$

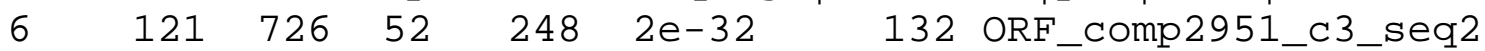

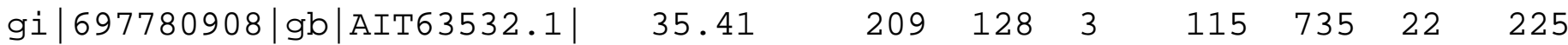

2e-32 132 ORF_comp2951_c3_seq2 gi|817516937|ref|WP_046572938.1|

$\begin{array}{llllllllll}41.43 & 210 & 115 & 3 & 112 & 738 & 80 & 282 & 3 e-32 & 133\end{array}$

ORF_comp2951_c3_seq2 gi|738342341|ref|WP_036294947.1| 39.32

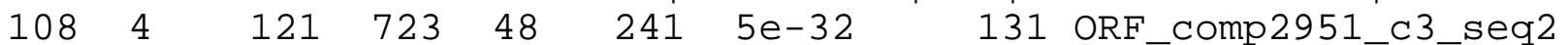

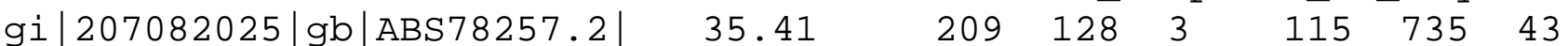

5e-32 131 ORF_comp2951_c3_seq2 gi|651604058|ref|WP_026598946.1|

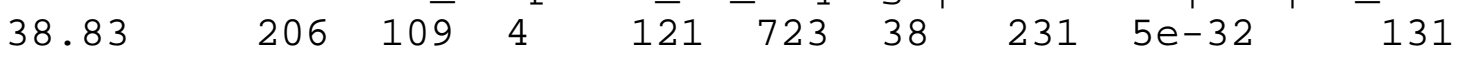

ORF_comp2951_c3_seq2 gi|737586499|ref|WP_035557439.1| 42.11

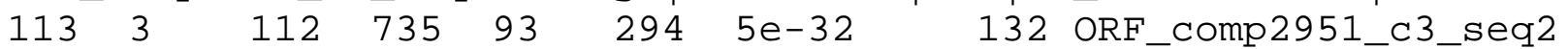

gi|165917203|gb|EDR35807.1| $37.23 \quad 188$ 113 $11 \quad 115 \quad 678 \quad 81$

8e-32 132 ORF_comp2951_c3_seq2 gi|489705594|ref|WP_003609731.1|

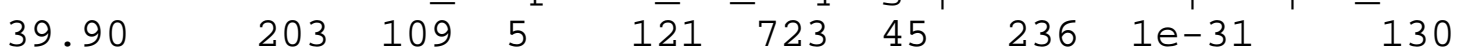

ORF_comp2951_c3_seq2 gi|917319449|ref|WP_051926161.1| 39.41

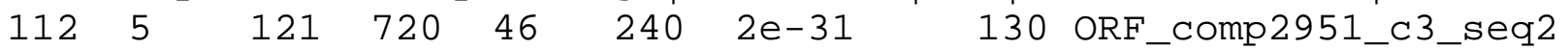

$\begin{array}{llllllll}\text { gi|427346779|gb|AFY29492.1| } & 39.02 & 205 & 116 & 4 & 112 & 720 & 25\end{array}$

3e-31 129 ORF_comp2951_c3_seq2 gi|759607566|ref|WP_043325766.1|

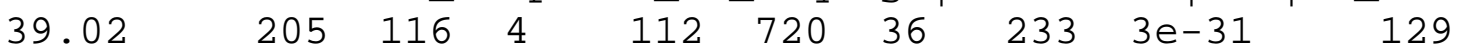

ORF_comp2951_c3_seq2 gi|662152692|ref|WP_030100516.1| 40.48

206

246

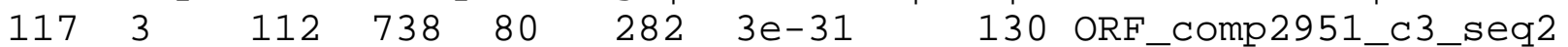

gi|654738300|ref|WP_028194554.1| $40.48 \quad 210 \quad 117 \quad 3 \quad r \begin{array}{lllll}112 & 738 & 80\end{array}$

282 4e-31 130 ORF_comp2951_c3_seq2 gi|797211671|ref|WP_045872614.1|

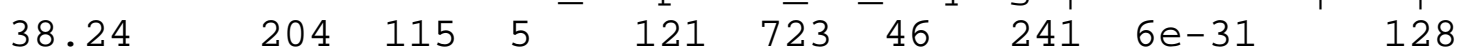

ORF_comp2951_c3_seq2 gi|981354997|ref|WP_059570263.1| 40.39

203

$\begin{array}{lllllll}112 & 4 & 121 & 723 & 40 & 235 & 1 \mathrm{e}-30\end{array}$

gi|916990632|ref|WP_051597344.1| $35.23 \quad 193 \quad 118 \quad 3 \quad r \begin{array}{lllll}157 & 735 & 58\end{array}$

243 8e-30 125 ORF_comp2951_c3_seq2 gi|928498313|gb|KPD18813.1|

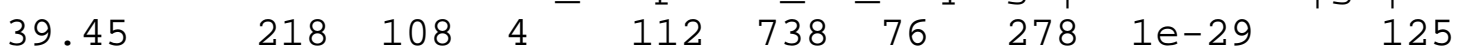

ORF_comp2951_c3_seq2 gi|504939174|ref|WP_015126276.1| 36.84

$\begin{array}{lllllll}121 & 418 & 738 & 53 & 252 & 1 \mathrm{e}-28 & 122 \\ \text { ORF_comp2951_c3_seq2 }\end{array}$

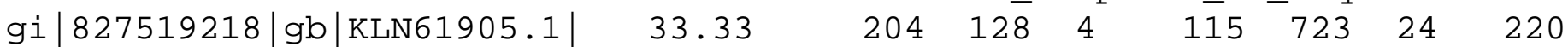

8e-28 119 ORF_comp2951_c3_seq2 gi|500232799|ref|WP_011902392.1|

$\begin{array}{llllllllll}34.98 & 203 & 120 & 6 & 121 & 720 & 38 & 231 & 2 \mathrm{e}-27 & 119\end{array}$

ORF_comp2951_c3_seq2 gi|920578875|ref|WP_053005650.1| 33.33

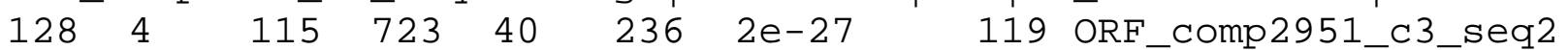

gi|167537519|ref|XP_001750428.1| $38.24 \quad 204 \quad 106 \quad 5 \quad r \begin{array}{lllll}121 & 702 & 48\end{array}$

2414 -27 118 ORF_comp2951_c3_seq2 gi|817130062|ref|WP_046502581.1|

$\begin{array}{llllllllll}33.33 & 204 & 128 & 4 & 115 & 723 & 40 & 236 & 1 \mathrm{e}-26 & 116\end{array}$

ORF_comp2951_c3_seq2 gi|1001837663|gb|KXS32259.1| 37.31 $201 \quad 117$

$5 \quad 127 \quad 723 \quad 46 \quad 239 \quad 1 \mathrm{e}-26 \quad 116$ ORF_comp2951_c3_seq2

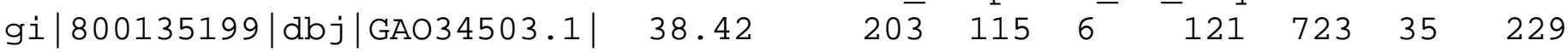

2e-26 115 ORF_comp2951_c3_seq2 gi|918692141|ref|WP_052561880.1|

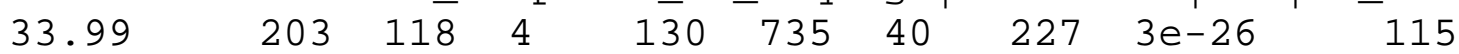

ORF_comp2951_c3_seq2 gi|925230922|gb|KOS75550.1| $41.18 \quad 170 \quad 85$

$4 \quad 229723 \quad 1 \quad 160 \quad 1 e-25 \quad 111$ ORF_comp2951_c3_seq2

gi|522080484|ref|WP_020591693.1| $33.33 \quad-204 \quad 128 \quad 4 \quad 115 \quad 723 \quad 36$

232 2e-25 113 ORF_comp2951_c3_seq2 gi|497652379|ref|WP_009966563.1| 
$37.44 \quad 203 \quad 95 \quad 4$

$\begin{array}{lllll}121 & 723 & 40 & 212 & 4 \mathrm{e}-25\end{array}$

112

ORF_comp2951_c3_seq2

gi $|772943476| \mathrm{gb}|\mathrm{AJX} 80078.1|$

37.44

20395

$4 \quad 121 \quad 723 \quad 30 \quad 202 \quad 5 e-25 \quad 111$ ORF_comp2951_c3_seq2

gi|748629167|ref|WP_039887344.1| $35.75 \quad-193 \quad 111 \quad 2 \quad 115 \quad 693 \quad 43$

222 7e-25 111 ORF_comp2951_c3_seq2 gi|497514158|ref|WP_009828356.1|

$\begin{array}{llllllllll}32.34 & 201 & 128 & 4 & 121 & 720 & 33 & 226 & 1 e-24 & 110\end{array}$

ORF_comp2951_c3_seq2 gi|748749862|ref|WP_040007977.1| 34.36

$1156 \quad 160 \quad 723 \quad 53 \quad 241 \quad 1 e-24 \quad 110$ ORF_comp2951_c3_seq2

gi|931478713|gb|KPK59845.1| $38.14 \quad 194 \quad 10 \overline{1} 4 \quad 4 \quad 121 \quad 702 \quad 26 \quad 200$

1e-24 110 ORF_comp2951_c3_seq2 gi|333458192|gb|EGK86811.1| 35.57

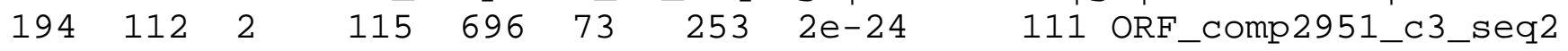

gi|1000089089|gb|KXJ56520.1| $33.66 \quad 202 \quad 126 \quad 4 \quad 121 \quad 723 \quad 34$

228

7e-24 108 ORF_comp2951_c3_seq2 gi|496167609|ref|WP_008892116.1|

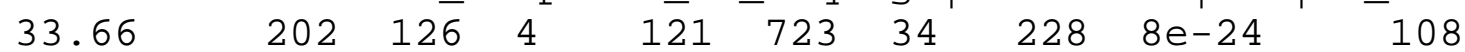

ORF_comp2951_c3_seq2 gi|966405784|ref|WP_058449190.1| 31.69

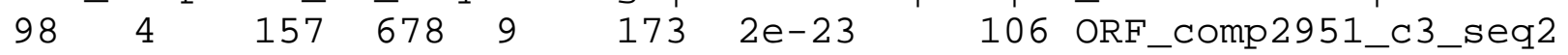

gi|915474789|ref|WP_050814322.1| $33.93 \quad 168 \quad 102 \quad 3 \quad r 229729 \quad 1$

160 7e-23 103 ORF_comp2951_c3_seq2 gi|290993528|ref|XP_002679385.1|

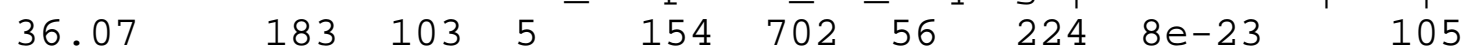

ORF_comp2951_c3_seq2 gi|518404331|ref|WP_019574538.1| 33.64

$1224 \quad 115735 \quad 56 \quad 256 \quad 1 e-22 \quad 105$ ORF_comp2951_c3_seq2

gi|692118754|ref|WP_032075052.1| $34.50 \quad 17 \overline{1} 105 \quad 3 \quad r 22 \quad 735 \quad 1$

166 2e-22 103 ORF_comp2951_c3_seq2 gi|308510244|ref|XP_003117305.1|

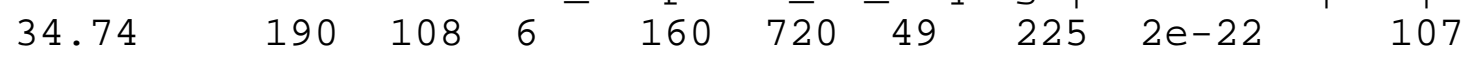

ORF_comp2951_c3_seq2 gi|213409680|ref|XP_002175610.1| 33.17

$120 \quad 6 \quad 121 \quad 72359 \quad 250 \quad 1 e-21 \quad 104$ ORF_comp2951_c3_seq2

gi|301112441|ref|XP_002997991.1| $33.67 \quad 19 \overline{9} 117 \quad 6 \quad 160 \quad 753 \quad 80$

264 1e-21 103 ORF_comp2951_c3_seq2 gi|599364119|ref|XP_007405638.1|

$\begin{array}{llllllllll}33.85 & 195 & 108 & 6 & 160 & 717 & 62 & 244 & 1 \mathrm{e}-21 & 102\end{array}$

ORF_comp2951_c3_seq2 gi|301112447|ref|XP_002997994.1| 34.39

189

$1115160 \quad 723 \quad 227403 \quad 1 e-21 \quad 105$ ORF_comp2951_c3_seq2

gi|193204637|ref|NP_001122616.1| $35.26 \quad 190 \quad 107 \quad 6 \quad r \begin{array}{lllll}160 & 720 & 41\end{array}$

217 1e-21 104 ORF_comp2951_c3_seq2

gi|14485518|gb|AAK62991.1|AF299332_1 $35.26 \quad 190 \quad 107 \quad 6 \quad 160 \quad 720$

$41 \quad 217$ 1e-21 103 ORF_comp2951_c3_seq2

gi|193204635|ref|NP_001122615.1| $35.26 \quad 190 \quad 107 \quad 6 \quad 160 \quad 720 \quad 49$

225 1e-21 104 ORF_comp2951_c3_seq2 gi|746710169|ref|WP_039669460.1|

$\begin{array}{llllllllll}33.92 & 171 & 106 & 3 & 229 & 735 & 1 & 166 & 2 \mathrm{e}-21 & 100\end{array}$

ORF_comp2951_c3_seq2 gi|676382929|ref|XP_009034743.1| 32.54

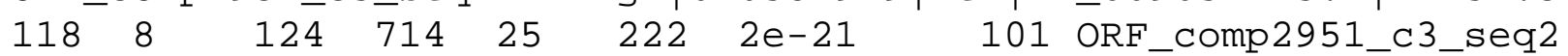

gi|325186679|emb|CCA21228.1| $34.92 \quad 189 \quad 11 \overline{0} \quad 5 \quad 160 \quad 723 \quad 71 \quad 247$

2e-21 102 ORF_comp2951_c3_seq2 gi|501565810|ref|WP_012570258.1|

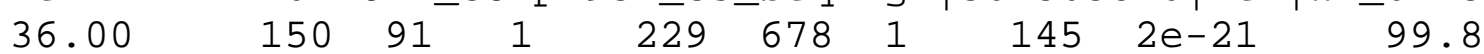

ORF_comp2951_c3_seq2 gi|309365040|emb|CAP23744.2| 35.26 $190 \quad 107$

$6 \quad 160 \quad 720 \quad 41 \quad 217 \quad 3 e-21 \quad 103$ ORF_comp2951_c3_seq2

gi|268531908|ref|XP_002631082.1| $35.26 \quad-190 \quad 107 \quad 6 \quad 160 \quad 720 \quad 41$

217 3e-21 102 ORF_comp2951_c3_seq2 gi|528895786|gb|EPZ35810.1|

$\begin{array}{llllllllll}32.67 & 202 & 118 & 7 & 154 & 750 & 48 & 234 & 3 e-21 & 103\end{array}$

ORF_comp2951_c3_seq2 gi|341888907|gb|EGT44842.1| 33.33 189112

$4 \quad 160 \quad 720 \quad 41 \quad 217 \quad 4 \mathrm{e}-21 \quad 103$ ORF_comp2951_c3_seq2

gi|551535405|ref|XP_005756498.1| $36.02 \quad 0 \quad 186 \quad 107 \quad 6 \quad 154 \quad 702 \quad 114$

290 4e-21 102 ORF_comp2951_c3_seq2 gi|695439924|ref|XP_009532833.1|

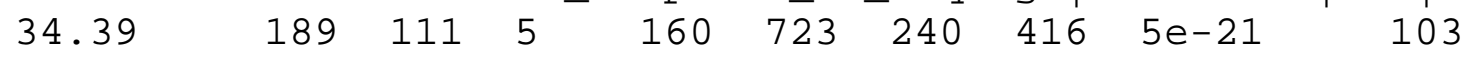

ORF_comp2951_c3_seq2 gi|872579356|ref|WP_048533013.1| 33.33

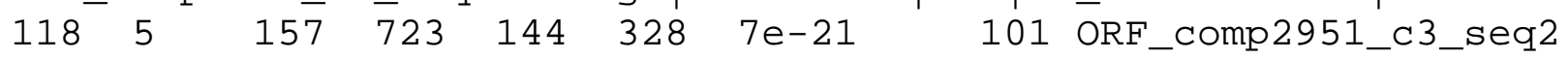

gi|813208513|dbj|GA050417.1| $33.33 \quad 180 \quad 107 \quad 3 \quad 154 \quad 693 \quad 146 \quad 312$

7e-21 103 ORF_comp2951_c3_seq2 gi|290980155|ref|XP_002672798.1| 
$\begin{array}{llllllllll}33.67 & 199 & 106 & 7 & 160 & 723 & 83 & 266 & 9 \mathrm{e}-21 & 102\end{array}$

ORF_comp2951_c3_seq2

gi|953489559|emb|CEG46925.1| 33.86

189112

$5 \quad 160723 \quad 188 \quad 364$ 9e-21 102 ORF_comp2951_c3_seq2

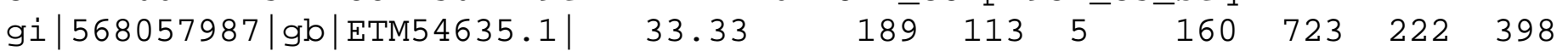

1e-20 102 ORF_comp2951_c3_seq2 gi|570996289|gb|ETP52930.1| 33.33

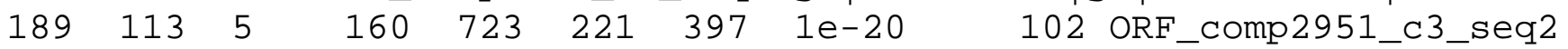

gi|675213985|ref|XP_008915877.1| $33.33 \quad 189 \quad 113 \quad 5 \quad 160 \quad 723 \quad 222$

398 1e-20 102 ORF_comp2951_c3_seq2 gi|570959958|gb|ETP24937.1|

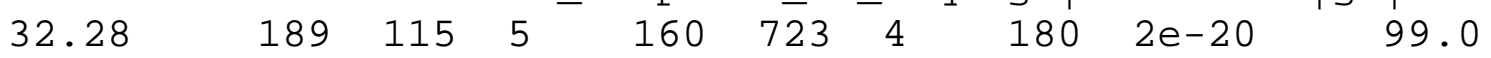

ORF_comp2951_c3_seq2 gi|970660098|gb|KUF97679.1| 33.33 $189 \quad 113$

$5 \quad 160 \quad 723 \quad 222398 \quad 2 e-20 \quad 101$ ORF_comp2951_c3_seq2

$\begin{array}{lllllllll}\text { gi|800137352|dbj|GA033130.1| } & 32.82 & 195 & 113 & 4 & 157 & 723 & 46 & 228\end{array}$

2e-20 98.6 ORF_comp2951_c3_seq2 gi|570335908|gb|ET083856.1|

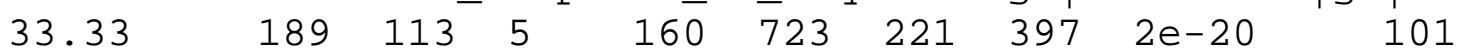

ORF_comp2951_c3_seq2 gi|544211776|ref|XP_005536287.1| 35.00

$1119 \quad 124 \quad 735 \quad 137 \quad 340 \quad 2 \mathrm{e}-20 \quad 102$ ORF_comp2951_c3_seq2

gi|33187146|gb|AA013810.2|AF384111_1 $34.56 \quad-217 \quad 123 \quad 8 \quad 115 \quad 750$

24226 2e-20 102 ORF_comp2951_c3_seq2

gi|737401579|ref|WP_035382755.1| $34.21 \quad 190 \quad 117 \quad 4 \quad 157 \quad 723 \quad 44$ 226 2e-20 98.2 ORF_comp2951_c3_seq2 gi|397628930|gb|EJK69115.1|

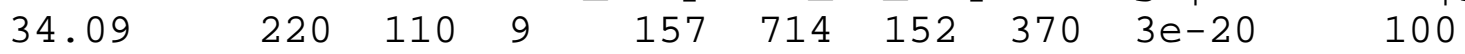

ORF_comp2951_c3_seq2 gi|675852512|ref|XP_009011816.1| 32.80

$114 \quad 4 \quad 160 \quad 723 \quad 114 \quad 290 \quad 3 e-20 \quad 100$ ORF_comp2951_c3_seq2

gi|566032216|gb|ETI55127.1| $32.28 \quad \begin{array}{lllllll}189 & 115 & 5 & 160 & 723 & 80 & 256\end{array}$

3e-20 99.4 ORF_comp2951_c3_seq2 gi|570335911|gb|ET083859.1|

$\begin{array}{llllllllll}32.28 & 189 & 115 & 5 & 160 & 723 & 80 & 256 & 3 \mathrm{e}-20 & 99.4\end{array}$

ORF_comp2951_c3_seq2 gi|570959955|gb|ETP24934.1| $32.80 \quad 189 \quad 114$

$\begin{array}{lllllllllll}5 & 160 & 723 & 222 & 398 & 3 e-20 & 100 & \text { ORF_comp2951_c3_seq2 } & & \\ \text { gi| } 568057990|\mathrm{gb}| \text { ETM54638.1| } & 32.28 & 189 & 115 & 5 & 160 & 723 & 80 & 256\end{array}$

4e-20 99.0 ORF_comp2951_c3_seq2 gi|570996278|gb|ETP52920.1|

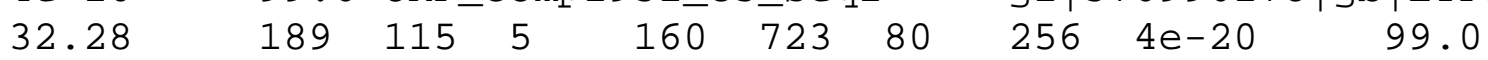

ORF_comp2951_c3_seq2 gi|675213991|ref|XP_008915880.1| 32.28

$1155160 \quad 723 \quad 80 \quad 256 \quad 4 \mathrm{e}-20 \quad 99.0$ ORF_comp2951_c3_seq2

gi|511010013|gb|EPB91244.1| $32.09 \quad 215 \quad 119 \quad 9 \quad 115 \quad 732 \quad 111 \quad 307$

5e-20 101 ORF_comp2951_c3_seq2 gi|758368534|emb|CEP09044.1| 32.09

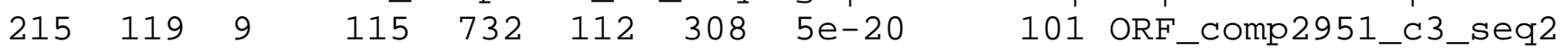

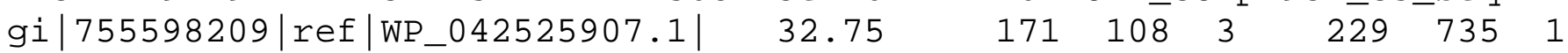

166 6e-20 95.9 ORF_comp2951_c3_seq2

gi|695064415|ref|XP_009420754.1| $33.17 \quad 208 \quad 120 \quad 6 \quad 115 \quad 723 \quad 22$

215 6e-20 100 ORF_comp2951_c3_seq2 gi|312072379|ref|XP_003139039.1|

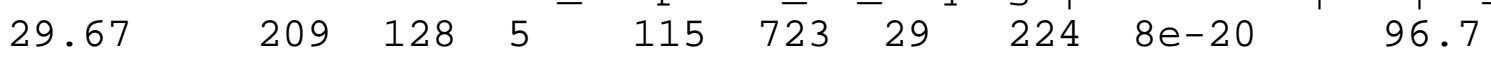

ORF_comp2951_c3_seq2 gi|758352771|dbj|GAN05107.1| $31.92 \quad 213 \quad 122$

$8 \quad 115732 \quad 116 \quad 312 \quad 8 \mathrm{e}-20 \quad 100$ ORF_comp2951_c3_seq2

gi|499786267|ref|WP_011467001.1| $29.57 \quad 230 \quad 131 \quad 7 \quad 121 \quad 738 \quad 93$

315 8e-20 98.2 ORF_comp2951_c3_seq2 gi|970651949|gb|KUF90697.1|

$\begin{array}{llllllllll}32.28 & 189 & 115 & 5 & 160 & 723 & 80 & 256 & 1 \mathrm{e}-19 & 97.8\end{array}$

ORF_comp2951_c3_seq2 gi|373405317|gb|AEY68568.1| 33.03 $218 \quad 123$

$8 \quad \begin{array}{llllll}121 & 753 & 24 & 225 & 1 \mathrm{e}-19 & 99.8 \text { ORF_comp2951_c3_seq2 }\end{array}$

gi|223995009|ref|XP_-002287188.1| 33.96

214 2e-19 95.5 ORF_comp2951_c3_seq2

gi $|760447765|$ ref|XP_011400951.1| 32.38

255 2e-19 98.2 ORF_comp2951_c3_seq2

gi|831778908|ref|XP_012755554.1| 29.47

260 2e-19 99.0 ORF_comp2951_c3_seq2

gi|694415950|ref|XP_009336117.1| 32.11

237 2e-19 99.0 ORF_comp2951_c3_seq2

gi|242051649|ref|XP_002454970.1| 32.88

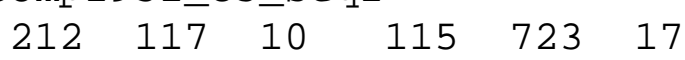

$\begin{array}{llllll}210 & 120 & 6 & 160 & 765 & 60\end{array}$

$\begin{array}{llllll}190 & 118 & 5 & 160 & 723 & 85\end{array}$

$\begin{array}{llllll}218 & 125 & 8 & 121 & 753 & 36\end{array}$

$\begin{array}{llllll}219 & 124 & 8 & 115 & 750 & 24\end{array}$ 
226 3e-19 99.๑ ORF_comp2951_c3_seq2 gi|694415952|ref|XP_009336118.1| 32.57 225 3e-19 98.6 ORF_comp2951_c3_seq2 gi|493073505|ref|WP_006122095.1| 29.33 252 3e-19 95.5 ORF_comp2951_c3_seq2 gi|676386555|ref|XP_009036556.1| 36.04 241 3e-19 95.5 ORF_comp2951_c3_seq2

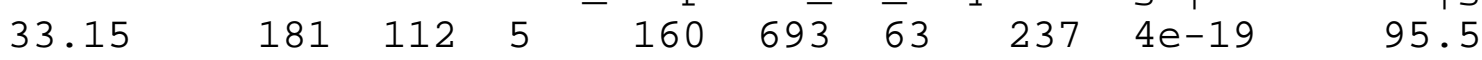
ORF_comp2951_c3_seq2 gi|635369185|emb|CCI42660.1| 32.80 $\begin{array}{llllll}218 & 124 & 8 & 121 & 753 & 24\end{array}$

$\begin{array}{llllll}225 & 135 & 6 & 115 & 723 & 30\end{array}$

$\begin{array}{llllll}197 & 111 & 5 & 151 & 723 & 54\end{array}$ gi $\mid 922867416$ | gb|K0034056 . 1| $5 \quad 16072355 \quad 231 \quad 4 \mathrm{e}-19 \quad 95.9$ ORF_comp2951_c3_seq2 gi|747072284|ref|XP_011083048.1| 34.33 224 4e-19 98.2 ORF_comp2951_c3_seq2 gi|490267384|ref|WP_004163951.1| 30.84 229 6e-19 94.7 ORF_comp2951_c3_seq2 gi|514776917|ref|XP_004968585.1| 31.51 226 6e-19 97.8 ORF_comp2951_c3_seq2 gi|747072282|ref|XP_011083047.1| 34.33 224 7e-19 98.2 ORF_comp2951_c3_seq2 gi 75213147 |sp|Q9SWW5 . 1|PCS1_WHEAT 34.33 224 8e-19 97.4 ORF_comp2951_c3_seq2

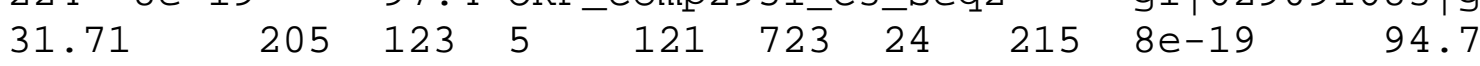
ORF_comp2951_c3_seq2 gi|702451810|ref|XP_010025777.1| 31.71 $1235121 \quad 723 \quad 41 \quad 232$ 9e-19 94.4 ORF_comp2951_c3_seq2 gi|490258075|ref|WP_004155296.1| $30.84 \quad 214 \quad 125 \quad 5 \quad \begin{array}{lllll}121 & 696 & 31\end{array}$ 243 9e-19 94.4 ORF_comp2951_c3_seq2 gi|326494126|dbj|BAJ85525.1| $\begin{array}{llllllllll}34.33 & 201 & 112 & 7 & 160 & 750 & 40 & 224 & 1 \mathrm{e}-18 & 97.1\end{array}$ ORF_comp2951_c3_seq2 gi|736937357|ref|WP_034934372.1| 29.60

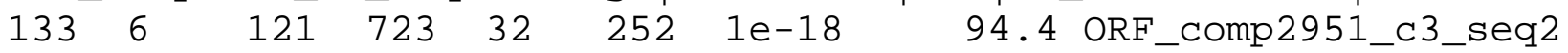
gi|907093431|gb|KNC99293.1| $32.26 \quad 217 \quad 113 \quad 6 \quad 154 \quad 747 \quad 98 \quad 299$ 1e-18 97.8 ORF_comp2951_c3_seq2

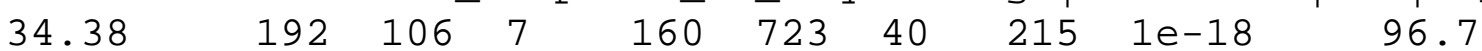
ORF_comp2951_c3_seq2 gi|443690998|gb|ELT92982.1| 28.64

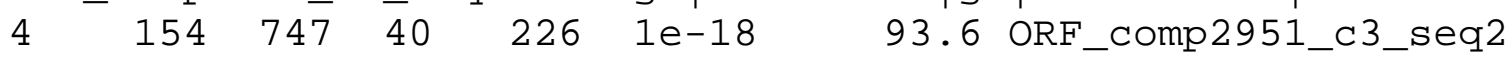
gi|901821666|gb|KMZ73511.1| $32.65 \quad 196 \quad 115 \quad 5 \quad \begin{array}{lllll}127 & 702 & 25 & 207\end{array}$ 2e-18 96.3 ORF_comp2951_c3_seq2 gi|727603973|ref|XP_010475030.1|

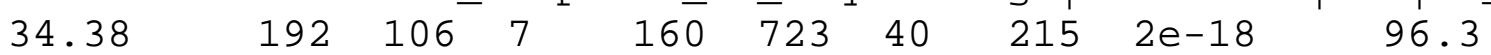
ORF_comp2951_c3_seq2 gi|752845383|ref|WP_041474394.1| 30.36 224 $1335 \quad 121 \quad 726 \quad 31 \quad 253 \quad 2 \mathrm{e}-18 \quad 93.6$ ORF_comp2951_c3_seq2 gi|541047267|gb|ERG86016.1| $32.98 \quad 191 \quad 109 \quad 6 \quad 160 \quad 723 \quad 32 \quad 206$ 2e-18 95.1 ORF_comp2951_c3_seq2 gi|551588562|ref|XP_005778952.1| $\begin{array}{llllllllll}31.09 & 193 & 121 & 5 & 160 & 723 & 12 & 197 & 2 \mathrm{e}-18 & 92.0\end{array}$ ORF_comp2951_c3_seq2 gi|407312484|gb|AFU06381.1| 33.66 $7 \quad 160750 \quad 40 \quad 224 \quad 2 \mathrm{e}-18 \quad 96.3$ ORF_comp2951_c3_seq2 gi|723731779|ref|XP_010326652.1| $32.84 \quad-201 \quad 115 \quad \overline{7} \quad 160 \quad 750 \quad 40$ 224 2e-18 95.9 ORF_comp2951_c3_seq2 gi|970053982|ref|XP_015088621.1| 32.84 224 2e-18 95.9 ORF_comp2951_c3_seq2

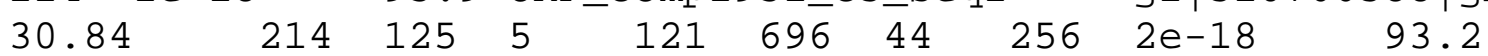
ORF_comp2951_c3_seq2 gi|970053984|ref|XP_015088622.1| 32.84

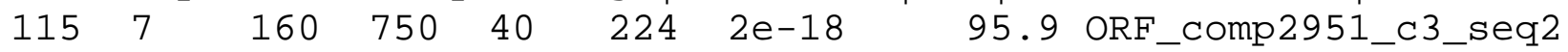
gi|460404089|ref|XP_004247517.1| $32.84 \quad 201 \quad 115 \quad 7 \quad 160 \quad 750 \quad 40$ 224 2e-18 95.9 ORF_comp2951_c3_seq2 gi|729701043|emb|CEJ०2028.1| $\begin{array}{llllllllll}29.58 & 213 & 127 & 8 & 115 & 732 & 57 & 253 & 3 \mathrm{e}-18 & 95.5\end{array}$ ORF_comp2951_c3_seq2 gi|28569702|emb|CAD68108.1| 33.33 $7 \quad 160 \quad 750 \quad 4 \quad 188$ 3e-18 95.5 ORF_comp2951_c3_seq2 gi|743942813|ref|XP_011015906.1| 30.62 215 3e-18 95.9 ORF_comp2951_c3_seq2

$\begin{array}{llllll}201 & 115 & 7 & 160 & 750 & 40\end{array}$ gi $\mid 310766388$ | gb|ADP11338.1| 
gi $\mid 972777756$ |ref|NP_001305597.1| 33.33 188 3e-18 95.5 ORF_comp2951_c3_seq2 gi|695071232|ref|XP_009382679.1| 31.66 224 3e-18 95.9 ORF_comp2951_c3_seq2 $\begin{array}{llllllll}33.85 & 192 & 107 & 7 & 160 & 723 & 40 & 215\end{array}$ ORF_comp2951_c3_seq2

$\begin{array}{lllllll}133 & 5 & 121 & 726 & 31 & 253 & 3 e-18\end{array}$ gi|531558322|gb|AGT57959.1| 33.33 3e-18 95.1 ORF_comp2951_c3_seq2 33.83 $201 \quad 113 \quad 7$

ORF_comp2951_c3_seq2 $160 \quad 750 \quad 40$ gi | 848932096 |ref|XP_012828972.1| $\begin{array}{llllll}199 & 120 & 5 & 160 & 750 & 40\end{array}$ gi | 325516458 |gb |ADZ24787 . 1 | 3e-18 95.5 ef WP_o41474048.1| 30.36

224 92.8 ORF_comp2951_c3_seq2 $\begin{array}{lllllll}195 & 116 & 5 & 160 & 738 & 51 & 233\end{array}$ gi | 848932098 | ref |XP_012828973 .1| $224 \quad 3 e-18 \quad 95.9$

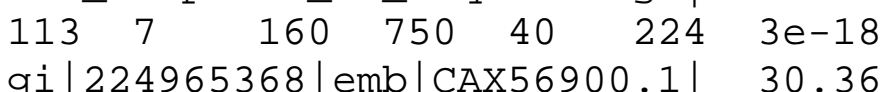
3e-18 92.8 ORF_comp2951_c3_seq2 $\begin{array}{lllllll}29.58 & 213 & 127 & 8 & 115 & 732 & 57\end{array}$ ORF_comp2951_c3_seq2

95.9 ORF_comp2951_c3 seq2 $\begin{array}{lllllll}224 & 133 & 5 & 121 & 726 & 44 & 266\end{array}$ gi |727146285 | emb |CEG71517.1| $253 \quad 4 \mathrm{e}-18 \quad 95.1$ gi| 568215457|ref |NP_001275308.1| 33.33 $\begin{array}{lllllll}114 & 7 & 160 & 750 & 40 & 224 & 4 \mathrm{e}-18\end{array}$ gi|857976683|emb|CE097478.1| 29.73 4e-18 94.7 ORF_comp2951_c3_seq2 33.83 $\begin{array}{llllll}201 & 113 & 7 & 160 & 750 & 40\end{array}$ 95.5 ORF_comp2951_c3_seq2

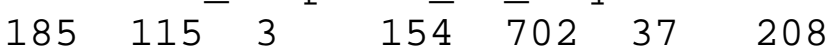
gi | 848932094 |ref |XP_012828971.1| $224 \quad 4 \mathrm{e}-18 \quad 95.9$ ORF_comp2951_c3_seq2 gi|1002820966|gb|AMN87031.1| 32.72 $217 \quad 127$ $7 \quad \begin{array}{lllll}115 & 750 & 24 & 226 & 4 \mathrm{e}-18 \quad 95.5 \text { ORF_comp2951_c3_seq2 }\end{array}$ gi|926791675|ref|XP_013904311.1| 40.16 125 4e-18 90.1 ORF_comp2951_c3_seq2 $33.51 \quad 191106 \quad 6$
ORF comp2951 c3 seq2 $\begin{array}{lllll}154 & 714 & 403 & 576 & 4 \mathrm{e}-18\end{array}$ $\begin{array}{llllll}122 & 71 & 2 & 361 & 723 & 5\end{array}$ gi | 672829171 | gb |KFH74060 . 1| $4 \mathrm{e}-18 \quad 95.9$

ORF_comp2951_c3_seq2 gi | 670428756 |ref|XP_008655225.1| 32.26 217

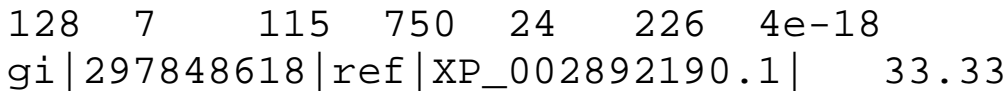
215 4e-18 94.4 ORF_comp2951_c3_seq2

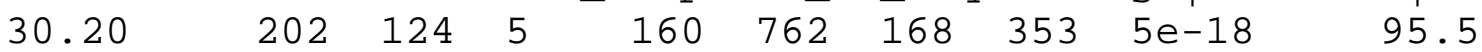
ORF_comp2951_c3_seq2 gi | 293337247 | ref | NP_001168641.1| 95.1 ORF_comp2951_c3_seq2

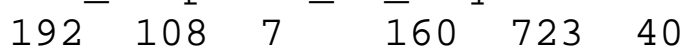
gi $|66803020|$ ref $\left|X P \_635353.1\right|$

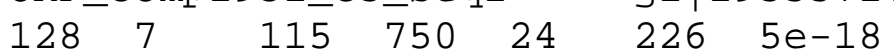
95.1 ORF_comp2951_c3_seq2

gi|302847090|ref|XP_002955080.1| 34.52 187 5e-18 90.9 ORF_comp2951_c3_seq2 gi|302782732|ref|XP_002973139.1| 31.71 224 6e-18 94.7 ORF_comp2951_c3_seq2 gi|302789570|ref|XP_002976553.1| 31.71 224 6e-18 94.7 ORF_comp2951_c3_seq2 33.33 1921087
ORF_comp2951_c3_seq2 $\begin{array}{lllll}160 & 723 & 40 & 215 & 6 \mathrm{e}-18\end{array}$ $16891 \quad 5 \quad 160 \quad 654 \quad 36$ 217 $\begin{array}{llllll}205 & 116 & 7 & 160 & 762 & 40\end{array}$ $\begin{array}{llllll}205 & 116 & 7 & 160 & 762 & 40\end{array}$ gi|674249977|gb|KFK42742.1| gi|312171048 | emb|CBX79307.1| 29.91 $214 \quad 127$ $5 \quad 121696 \quad 17 \quad 229$ 6e-18 91.7 ORF_comp2951_c3_seq2 gi|1002820968|gb|AMN87032.1| 31.51 7e-18 94.7 ORF_comp2951_c3_seq2 $\begin{array}{llll}30.41 & 217 & 113 & 6 \\ \text { ORF comp2951 c3 seq2 }\end{array}$ $\begin{array}{llllll}10 & 115 & 723 & 57 & 300 & 8 \mathrm{e}-18\end{array}$ $\begin{array}{lll}115 & 723 & 17\end{array}$ $\begin{array}{llllll}219 & 127 & 8 & 115 & 750 & 24\end{array}$ 226 gi |552916579|gb|ESA01781.1| $2098 \mathrm{e}-18 \quad 90.9$ gi|444891677|gb|AGE13358.1| 30.59 255114 gi|478729310|emb|CCP05801.1| 29.91 9e-18 91.3 ORF_comp2951_c3_seq2 32.82 $\begin{array}{llllll}195 & 111 & 6 & 157 & 696 & 93\end{array}$ 92.8 ORF_comp2951_c3_seq2

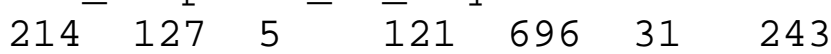
gi | 224004956 | ref |XP_002296129.1| $\begin{array}{lll}282 & 1 e-17 & 92.4\end{array}$

ORF_comp2951_c3_seq2 gi |470239215 |ref|XP_004351373.1| 30.56 216 $\begin{array}{lllllll}128 & 8 & 127 & 762 & 380 & 577 & 1 \mathrm{e}-17\end{array}$ gi|661175740|emb|CDH61092.1| 32.80 1e-17 94.4 ORF_comp2951_c3_seq2 94.7 ORF_comp2951_c3_seq2 $\begin{array}{lllllll}189 & 112 & 5 & 160 & 723 & 119 & 293\end{array}$ gi | 490272265 |ref |WP_004168384.1|

29.91 $214 \quad 127 \quad 5$ $\begin{array}{lllll}121 & 696 & 31 & 243 & 1 \mathrm{e}-17\end{array}$ 91.3

ORF_comp2951_c3_seq2 gi|159485978|ref|XP_001701021.1| 32.53

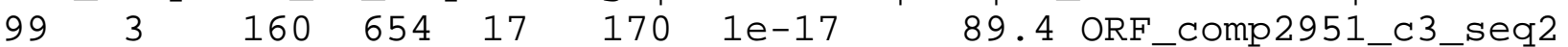

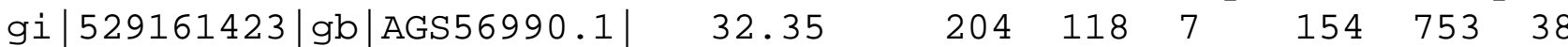


ORF_comp2951_c3_seq2

gi|113594574|dbj|BAF18448.1| 31.82

198121

$4 \quad 160750 \quad 41 \quad 225$ 1e-17 90.9 ORF_comp2951_c3_seq2

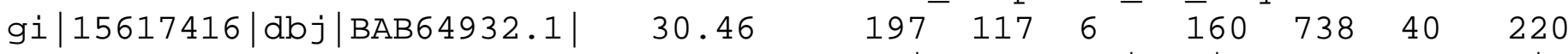

1e-17 94.0 ORF_comp2951_c3_seq2

gi|552820766 |ref|XP_005845668.1|

30.29

$175 \quad 107 \quad 4$

$154 \quad 675 \quad 47$

$2071 \mathrm{e}-17$ 90.1

ORF_comp2951_c3_seq2 gi|515351240|ref|WP_016863694.1| 30.46

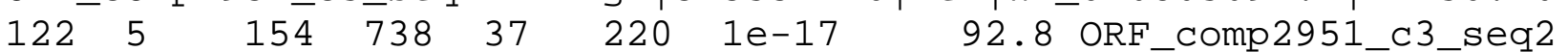

gi $|671683790| \mathrm{emb}|\mathrm{CDS} 12824.1| \quad 32.80$

1e-17 94.0 ORF_comp2951_c3_seq2

27.85 $237 \quad 133 \quad 7$

$115 \quad 732 \quad 40$

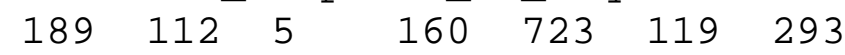
gi | 916690104 | ref |WP_051297195.1| $269 \quad 1 \mathrm{e}-17 \quad 90.9$

ORF_comp2951_c3_seq2 gi|502816264|ref|WP_013051240.1| 30.22

225

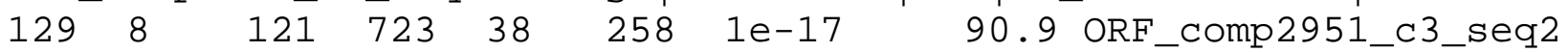

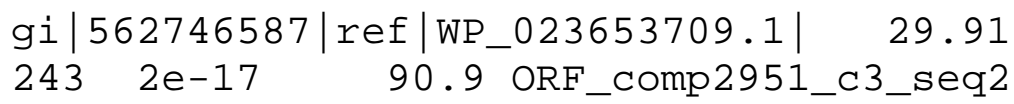

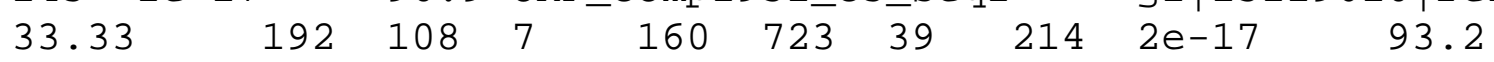

ORF_comp2951_c3_seq2 gi|743835874|ref|XP_010935936.1| 33.51

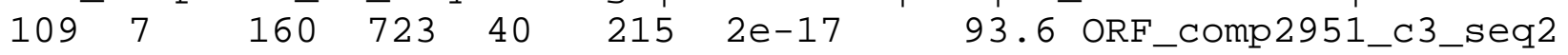

gi|672825823|gb|KFH70714.1| $33.33 \quad 201 \quad 114 \quad 6 \quad 160 \quad 756 \quad 148 \quad 330$

2e-17 94.0 ORF_comp2951_c3_seq2 gi|505014688|ref|WP_015201790.1|

$\begin{array}{llllllllll}29.83 & 181 & 113 & 4 & 154 & 693 & 37 & 204 & 2 \mathrm{e}-17 & 92.8\end{array}$

ORF_comp2951_c3_seq2 gi|727439853|ref|XP_010501307.1| 33.85

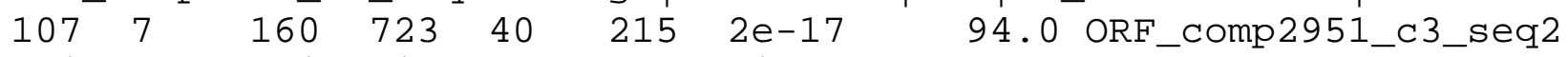

192

gi|727439853|ref|XP_010501307.1| $33.71 \quad 178 \quad 98 \quad 7 \quad \begin{array}{llll}7202 & 723 & 259\end{array}$

$4205 e-14 \quad 83.6$ ORF_comp2951_c3_seq2

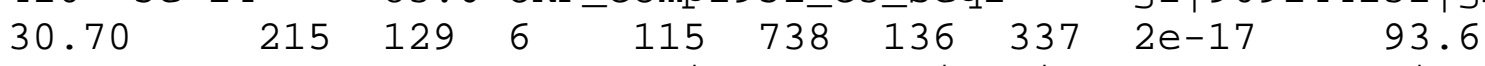

ORF_comp2951_c3_seq2 gi|550281002|ref|WP_022607401.1| 33.52

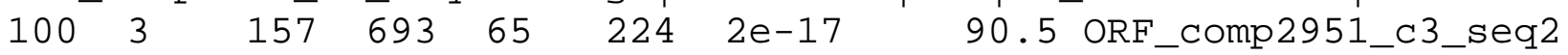

gi|734553453|gb|KHN80812.1| $30.77 \quad \begin{array}{lllllll}195 & 121 & 4 & 160 & 738 & 43 & 225\end{array}$

2e-17 92.0 ORF_comp2951_c3_seq2

$28.70 \quad 223 \quad 135 \quad 6 \quad 121 \quad 723 \quad 32 \quad 252 \quad 2 \mathrm{e}-17 \quad 9.1$

ORF_comp2951_c3_seq2 gi|971557981|ref|XP_015166074.1| 32.84

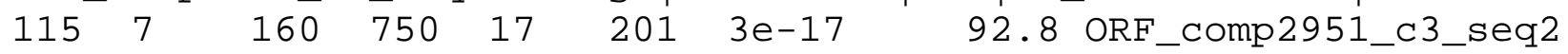

gi|971557978|ref|XP_015166073.1| $32.84 \quad 201 \quad 115 \quad 7 \quad \begin{array}{lllll}160 & 750 & 40\end{array}$

224 3e-17 92.8 ORF_comp2951_c3_seq2

gi $|122202937| \mathrm{sp}|\mathrm{Q} 2 \mathrm{QKL} 5 . \overline{1}|$ PCS3_LOTJA 30.00

$22 \quad 2153 e-17 \quad 92.4$ ORF_comp2951_c3_seq2

\begin{tabular}{ll|r|} 
gi $|330794008| r e f\left|X P \_003285073.1\right|$ & 29.35 \\
$2613 e-17$ & 92.8 & ORF_comp2951_c3_seq2
\end{tabular}

$\begin{array}{lll}2613 e-17 & 92.8 & \text { ORF_comp2951_c3_seq2 } \\ \text { gi }|224129604| \text { ref|XP_o02320627.1| 30.35 }\end{array}$

207 3e-17 92.8 ORF_comp2951_c3_seq2

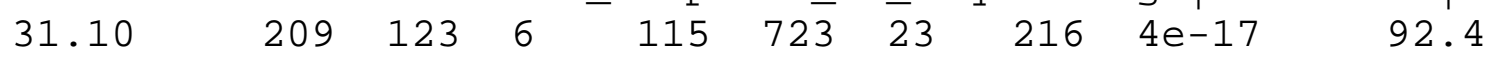

ORF_comp2951_c3_seq2 gi|444891679|gb|AGE13359.1| 32.31 195112

$6 \quad 157696 \quad 93 \quad 282$ 4e-17 90.9 ORF_comp2951_c3_seq2

gi|19114464|ref|NP_593552.1| $28.27 \quad 191 \quad 123 \quad 5 \quad \begin{array}{lllll}154 & 723 & 73 & 250\end{array}$

4e-17 91.7 ORF_comp2951_c3_seq2

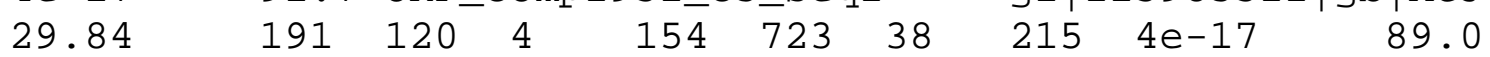

ORF_comp2951_c3_seq2 gi|909141466|gb|KNE66257.1| 32.80

$4 \quad \begin{array}{llllll}160 & 723 & 87 & 262 & 4 \mathrm{e}-17 & 92.8 \text { ORF_comp2951_c3_seq2 }\end{array}$

gi|676493293|ref|XP_009066193.1| $29.67 \quad \begin{array}{llllll}182 & 114 & 4 & 160 & 702 & 34\end{array}$

202 4e-17 89.0 ORF_comp2951_c3_seq2

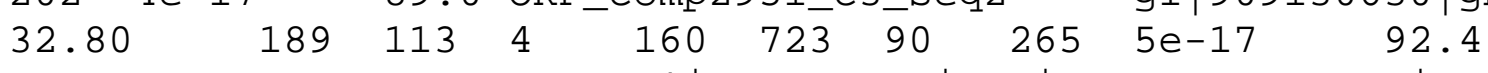

ORF_comp2951_c3_seq2 gi|743869439|ref|XP_010905812.1| 30.92

$122 \quad 6 \quad 121 \quad 723 \quad 24 \quad 215 \quad 5 e-17 \quad 90.5$ ORF_comp2951_c3_seq2

gi|731346799|ref|XP_010684646.1| $31.43 \quad 210 \quad 121 \quad 8 \quad \begin{array}{cccc}115 & 723 & 23\end{array}$

216 6e-17 92.0 ORF_comp2951_c3_seq2 
gi|676430709|ref|XP_009046015.1| 29.63

$2226 \mathrm{e}-17$ 88.6 ORF_comp2951_c3_seq2

gi $|331218210|$ ref|XP_003321783.1| 31.31

364 6e-17 90.9 ORF_comp2951_c3_seq2 $\begin{array}{llllll}189 & 120 & 4 & 160 & 723 & 46\end{array}$

$\begin{array}{llllll}198 & 117 & 5 & 154 & 723 & 178\end{array}$ gi | 902234844 | gb|KNA23610.1|

6e-17 92.0

ORF_comp2951_c3_seq2 gi|50659119|gb|AAT80341.1| $31.16 \quad 199 \quad 114$

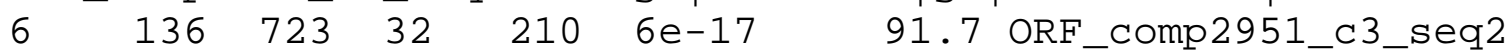

gi|388497750|gb|AFK36941.1| $30.00 \quad 210 \quad 124 \quad 7 \quad \begin{array}{lllll}115 & 723 & 22 & 215\end{array}$ 7e-17 91.7 ORF_comp2951_c3_seq2 gi|659119977|ref|XP_008459945.1|

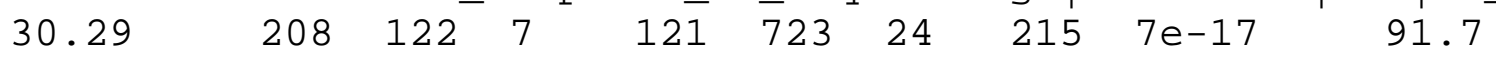

ORF_comp2951_c3_seq2 gi|573946675|ref|XP_006655670.1| 31.31 $1224 \quad 160 \quad 750 \quad 42 \quad 226 \quad 8 \mathrm{e}-17 \quad 91.7$ ORF_comp2951_c3_seq2 gi|698514101|ref|XP_o09801957.1| 33.33
$2248 \mathrm{e}-17 \quad 91.7$ ORF comp2951 c3 seq2 gi|567155800|ref|XP_006418181.1| 32.29 179 8e-17 90.9 ORF_comp2951_c3_seq2 gi|331218212|ref|XP_003321784.1| 32.02 239 8e-17 90.9 ORF_comp2951_c3_seq2 $\begin{array}{lllllll}31.25 & 192 & 116 & 5 & 154 & 723 & 120\end{array}$ $201 \quad 114 \quad 7 \quad 160 \quad 750 \quad 40$

$\begin{array}{llllll}192 & 110 & 7 & 160 & 723 & 4\end{array}$

$\begin{array}{llllll}203 & 111 & 5 & 154 & 723 & 51\end{array}$ gi $|1001614567| \mathrm{gb} \mid \mathrm{KXS18316.1|}$ ORF_comp2951_c3_seq2 gi | 698514104 |ref |XP_009801958.1| 33.33 201 $1147 \quad 160 \quad 750 \quad 40 \quad 224 \quad 8 \mathrm{e}-17 \quad 91.7$ ORF_comp2951_c3_seq2 gi|504984674|ref|WP_015171776.1| 29.89 209 8e-17 90.5 ORF_comp2951_c3_seq2 gi $\mid 156379434$ |ref|XP_001631462.1| 25.95 213 9e-17 88.2 ORF_comp2951_c3_seq2 $33.33 \quad 201 \quad 114 \quad 7$
ORF_comp2951_c3_seq2

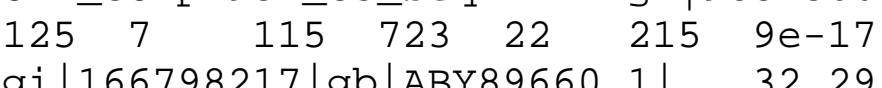
$\begin{array}{llr}\text { gi|166798217|gb|ABY89660.1| } 32.29 \\ 9 \mathrm{e}-17 & 91.3 \text { ORF comp2951 c3 seq2 }\end{array}$

183 108_ 4 ( 160 $31.69 \quad 1831084$
ORF_comp2951_c3_seq2 $160 \quad 702 \quad 39 \quad 206 \quad 9 \mathrm{e}-17 \quad 91.3$

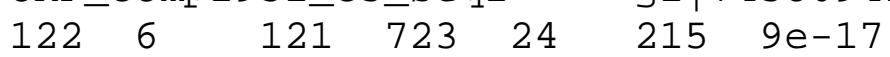
gi|734345461|gb|KHN10759.1| 30.62 9e-17 90.9 ORF_comp2951_c3_seq2

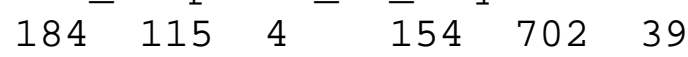
$\begin{array}{llllll}185 & 122 & 3 & 160 & 702 & 40\end{array}$ gi|768677235 |gb|AJU57239.1| $2249 e-17 \quad 91$. $2249 \mathrm{e}-17 \quad 91.3$ 29.52 210 91.3 ORF_comp2951_c3_seq2

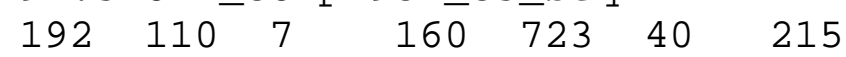
gi|502110105|ref|XP_004493800.1|

gi|743869429|ref|XP_010905810.1| 30.92

207

91.3 ORF_comp2951_c3_seq2

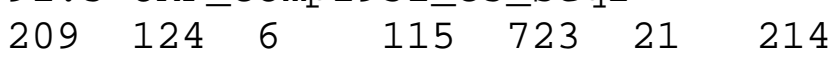
29.52 gi|641841507|gb|KD060419.1

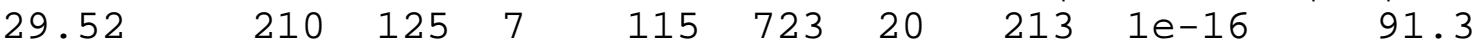
ORF_comp2951_c3_seq2 gi|923123265|ref|XP_013755101.1| 32.18 $1198 \quad 160 \quad 747 \quad 39 \quad 228 \quad 1 \mathrm{e}-16 \quad 90.9$ ORF_comp2951_c3_seq2 gi|567185112|ref|XP_006403145.1| $34.38 \quad 192 \quad 106 \quad 7 \quad \begin{array}{lllll}160 & 723 & 40\end{array}$ 215 1e-16 91.3 ORF_comp2951_c3_seq2 gi|21104516|dbj|BAB93119.1|

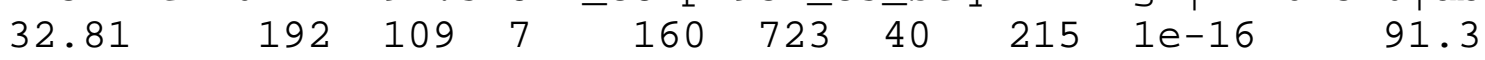
ORF_comp2951_c3_seq2 gi|961088958|ref|XP_014771294.1| 32.46 $1116 \quad 160 \quad 723 \quad 39 \quad 214 \quad 1 e-16 \quad 90.9$ ORF_comp2951_c3_seq2 gi|674895889|emb|CDY36967.1| 30.37 1e-16 90.5 ORF_comp2951_c3_seq2 $29.89 \quad 174 \quad 1074$
ORF_comp2951_c3_seq2 $160 \quad 678 \quad 44$

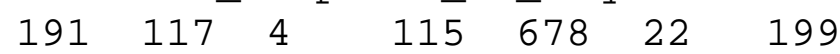
191 gi|302843401|ref|XP_002953242.1| gi $\mid 567902606$ |ref|XP_006443791.1| 29.52 210 $\begin{array}{lllllll}125 & 7 & 115 & 723 & 22 & 215 & 1 \mathrm{e}-16\end{array}$ gi|641841510|gb|KD060422.1| 33.68 1e-16 91.3 ORF_comp2951_c3_seq2 $\begin{array}{lllllll}30.62 & 209 & 124 & 6 & 115 & 723 & 23\end{array}$ 91.3 ORF_comp2951_c3_seq2 $19310877 \quad 160 \quad 726 \quad 40 \quad 216$ gi | 950949688 |ref|XP_014495217.1| 216 1e-16 90.9 ORF_comp2951_c3_seq2 gi|922429656|ref|XP_013620881.1| 33.33 $10877 \quad 160 \quad 723 \quad 40 \quad 215 \quad 1 \mathrm{e}-16 \quad 90.9$ ORF_comp2951_c3_seq2 gi $\mid 674936482$ | emb |CDX96939.1| 33.33 1e-16 90.9 ORF_comp2951_c3_seq2 33.33 $192108 \quad 7$ ORF_comp2951_c3_seq2 $160 \quad 723 \quad 40$

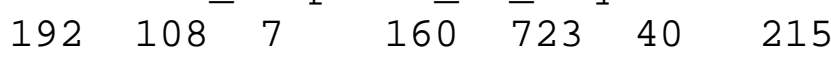
gi | 685383195 | ref |XP_009123930.1 | $215 \quad 1 \mathrm{e}-16 \quad 90.9$ gi|675852508|ref|XP_009011814.1| 31.84 
$\begin{array}{lllllll}122 & 5 & 154 & 750 & 80 & 267 & 1 \mathrm{e}-16\end{array}$ 90.9 ORF_comp2951_c3_seq2 gi | 27448224 |gb|AA013809.1|AF384110_1 32.81 $\begin{array}{llll}192 & 109 & 7 & 160\end{array}$

723

$40 \quad 2151 \mathrm{e}-16 \quad 90.9$ ORF_comp2951_c3_seq2

gi|922429654|ref|XP_013620880.1| 33.33

$\begin{array}{llllll}192 & 108 & 7 & 160 & 723 & 40\end{array}$

215 1e-16 90.9 ORF_comp2951_c3_seq2 gi|923864531|ref|XP_013707638.1| 33.33 $2151 \mathrm{e}-16$ 90.9 ORF_comp2951_c3_seq2 gi|567155803|ref|XP_006418182.1| 32.29 215 1e-16 90.5 ORF_comp2951_c3_seq2 gi|685951299|ref|XP_009269521.1| 31.22 231 1e-16 90.1 ORF_comp2951_c3_seq2 gi|568851639|ref|XP_006479495.1| 33.68 216 1e-16 90.9 ORF_comp2951_c3_seq2 gi|922563714|ref|XP_013610130.1| 30.57 201 1e-16 90.9 ORF_comp2951_c3_seq2 gi|694387379|ref|XP_009369444.1| 32.35 $2251 \mathrm{e}-16 \quad 90.9$ ORF_comp2951_c3_seq2

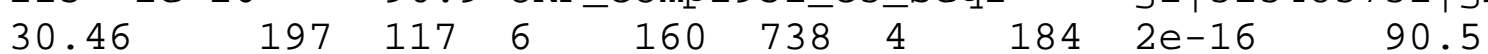
ORF_comp2951_c3_seq2 gi|763775184|gb|KJB42307.1| 32.81

$\begin{array}{llllll}192 & 108 & 7 & 160 & 723 & 40\end{array}$

$\begin{array}{llllll}192 & 110 & 7 & 160 & 723 & 40\end{array}$

$\begin{array}{llllll}189 & 115 & 5 & 160 & 723 & 57\end{array}$

$\begin{array}{llllll}193 & 108 & 7 & 160 & 726 & 40\end{array}$

$\begin{array}{llllll}193 & 114 & 5 & 115 & 678 & 24\end{array}$

$\begin{array}{llllll}204 & 118 & 7 & 154 & 753 & 38\end{array}$ gi | 313483731 |gb|ADR51694 . 1 | $7 \quad 160723 \quad 42 \quad 217$ 2e-16 88.6 ORF_comp2951_c3_seq2 gi $|567902610|$ ref|XP_006443793.1| 30.20 207 2e-16 90.5 ORF_comp2951_c3_seq2 gi $|685326730|$ ref|XP_009101739.1| 31.58 215 2e-16 90.5 ORF_comp2951_c3_seq2

30.26 1951205

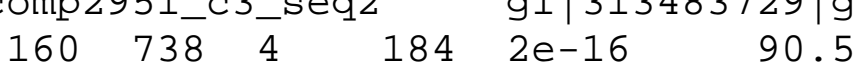
gi|891585491|ref|XP_013022696.1|

$\begin{array}{llllll}202 & 119 & 6 & 115 & 702 & 22\end{array}$ gi $|1002277234|$ ref|XP_015642955.1| 31.82 90.1 ORF_comp2951_c3_seq2 225 2e-16 90.5 ORF_comp2951_c3_seq2 gi|4768281|gb|AAD29446.1|AF085231_2 30.77 $45 \quad 232$ 2e-16 90.5 ORF_comp2951_c3_seq2 gi|470107265|ref|XP_004289969.1| 30.73 225 2e-16 90.5 ORF_comp2951_c3_seq2

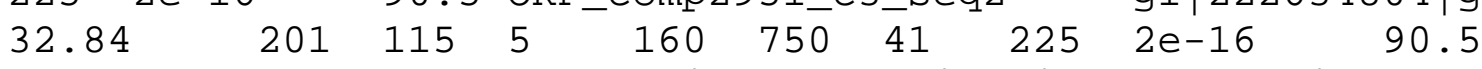

ORF_comp2951_c3_seq2 gi|661892612|emb|CDP03770.1| 34.03

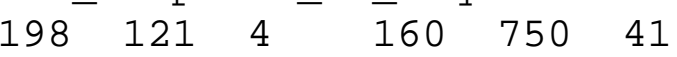

$6 \quad 160723 \quad 40 \quad 215 \quad 2 \mathrm{e}-16 \quad 90.5$ ORF_comp2951_c3_seq2

gi $|764527045|$ ref|XP_011458022.1| 30.73

229 2e-16 90.5 ORF_comp2951_c3_seq2

gi 672113326 |ref|XP_008810429.1| 32.98

215 2e-16 90.5 ORF_comp2951_c3_seq2

gi|595795184|ref|XP_007200974.1| 32.35

224 2e-16 90.5 ORF_comp2951_c3_seq2

gi|891559693|ref|XP_013018242.1| 27.75

251 2e-16 89.7 ORF_comp2951_c3_seq2

32. $29 \quad 192 \quad 110$ $\begin{array}{llllll}160 & 723 & 40 & 215 & 2 \mathrm{e}-16 & 90.5\end{array}$

ORF_comp2951_c3_seq2 gi|567902612 |ref |XP_

gi|593799054 | ref|XP_007162565.1| 31.10

216 2e-16 90.1 ORF_comp2951_c3_seq2

gi|985451243|ref|XP_015386353.1| 33.68

216 2e-16 90.5 ORF_comp2951_c3_seq2

$33.68 \quad 193 \quad 108 \quad 7$

ORF_comp2951_c3_seq2

218128

$\begin{array}{lll}121 & 753 \quad 28\end{array}$

$\begin{array}{llllll}191 & 110 & 7 & 160 & 723 & 40\end{array}$

$\begin{array}{llllll}204 & 118 & 7 & 154 & 753 & 37\end{array}$

$\begin{array}{llllll}191 & 124 & 5 & 154 & 723 & 74\end{array}$ gi | 18958247 | dbj |BAB85602 . 1| ORF comp2951 33.68 $209123 \quad 6 \quad 115723 \quad 23$

$\begin{array}{llllll}193 & 108 & 7 & 160 & 726 & 40\end{array}$ gi | 641841512 |gb|KD060424.1| $2 e-16 \quad 90.5$ $1196 \quad 115 \quad 702 \quad 22 \quad 207 \quad 2$ - 626 90.5 ORF_comp2951_c3_seq2 gi $|303281270|$ ref|XP_003059927.1| 33.66 220 2e-16 87.0 ORF_comp2951_c3_seq2 30.26 $\begin{array}{lllllll}195 & 120 & 5 & 160 & 738 & 4 & 184\end{array}$

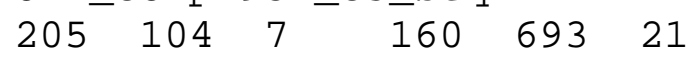
gi | 313483727 | gb |ADR51692 . 1 | $2 e-16 \quad 90.1$ 
ORF_comp2951_c3_seq2

gi|313483717|gb|ADR51687.1| 30.26

195120

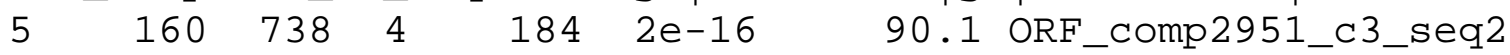

gi|923847316|ref|XP_013702842.1| $31.77 \quad-\begin{array}{llllll}192 & 111 & \overline{7} & 160 & 723 & 40\end{array}$

215 2e-16 88.2 ORF_comp2951_c3_seq2 gi|641841509|gb|KD060421.1|

$\begin{array}{llllllllll}33.68 & 193 & 108 & 7 & 160 & 726 & 40 & 216 & 2 \mathrm{e}-16 & 90.5\end{array}$

ORF_comp2951_c3_seq2 gi|313483733|gb|ADR51695.1| 30.46 $197 \quad 117$

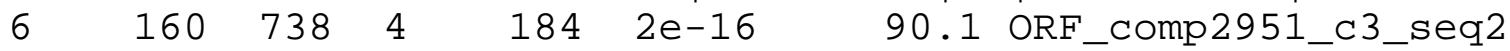

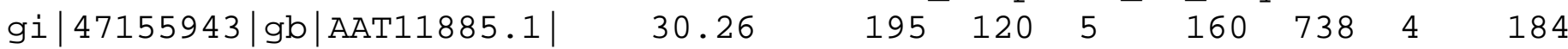

2e-16 90.1 ORF_comp2951_c3_seq2 gi|641841508|gb|KD060420.1|

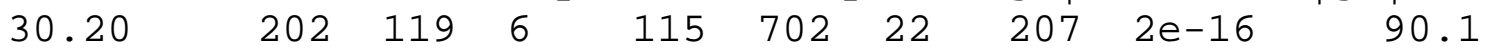

ORF_comp2951_c3_seq2 gi|697171098|ref|XP_009594480.1| 32.84

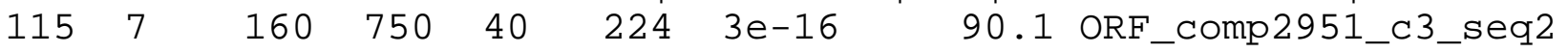

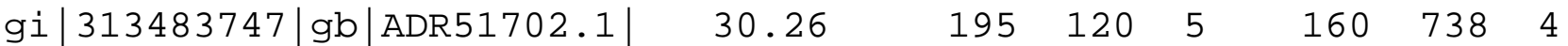

3e-16 89.7 ORF_comp2951_c3_seq2

$\begin{array}{llllllllll}30.26 & 195 & 120 & 5 & 160 & 738 & 4 & 184 & 3 e-16 & 89.7\end{array}$

ORF_comp2951_c3_seq2 gi|313483751|gb|ADR51704.1| 30.46

197117

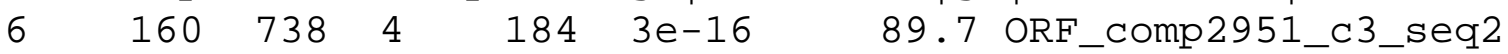

gi|313483713|gb|ADR51685.1| $30.26 \quad 195 \quad 120 \quad 5 \quad 160 \quad 738 \quad 4 \quad 184$

3e-16 89.7 ORF_comp2951_c3_seq2 gi|313483711|gb|ADR51684.1|

$\begin{array}{llllllllll}30.26 & 195 & 120 & 5 & 160 & 738 & 4 & 184 & 3 e-16 & 89.7\end{array}$

ORF_comp2951_c3_seq2 gi|763775185|gb|KJB42308.1| 32.81 $192 \quad 109$

$7 \quad 160 \quad 723 \quad 42 \quad 217$ 3e-16 89.0 ORF_comp2951_c3_seq2

gi|356505041|ref|XP_003521301.1| $30.62 \quad 209 \quad 124 \quad 6 \quad 115 \quad 723 \quad 21$

214 3e-16 90.1 ORF_comp2951_c3_seq2 gi|313483725|gb|ADR51691.1|

$\begin{array}{llllllllll}30.46 & 197 & 117 & 6 & 160 & 738 & 4 & 184 & 3 e-16 & 89.7\end{array}$

ORF_comp2951_c3_seq2 gi|313483715|gb|ADR51686.1| 30.46 $197 \quad 117$

$\begin{array}{lllllll}6 & 160 & 738 & 4 & 184 & 3 \mathrm{e}-16 & 89.7 \text { ORF_comp2951_c3_seq2 }\end{array}$

gi|657981621|ref|XP_008382837.1| $32.84 \quad 204 \quad 117 \quad 7 \quad 34 \quad 753 \quad 38$

225 3e-16 89.7 ORF_comp2951_c3_seq2 gi|674877470|emb|CDY54482.1|

$\begin{array}{llllllllll}31.77 & 192 & 111 & 7 & 160 & 723 & 40 & 215 & 3 e-16 & 89.7\end{array}$

ORF_comp2951_c3_seq2 gi|313483721|gb|ADR51689.1| 30.46

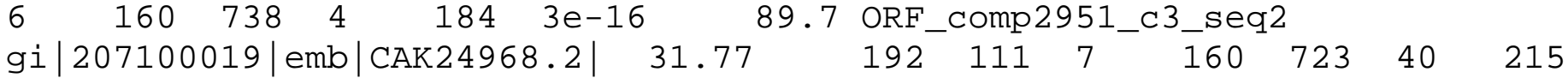

3e-16 89.7 ORF_comp2951_c3_seq2 gi|13928024|emb|CAC37692.1|

$\begin{array}{llllllllll}31.77 & 192 & 111 & 7 & 160 & 723 & 40 & 215 & 3 \mathrm{e}-16 & 89.7\end{array}$

ORF_comp2951_c3_seq2 gi|676476523|ref|XP_009060820.1| 28.02

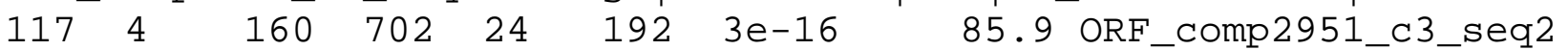

gi|923885023|ref|XP_013714028.1| $29.53 \quad 193 \quad 116 \quad 5 \quad 115 \quad 678 \quad 22$

199 3e-16 89.7 ORF_comp2951_c3_seq2 gi|475597402|gb|EMT23611.1|

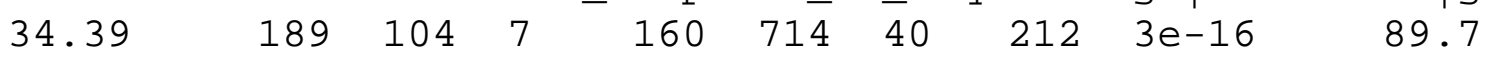

ORF_comp2951_c3_seq2 gi|170594708|ref|XP_001902100.1| 31.43

$1107 \quad 160 \quad 738 \quad 44 \quad 236 \quad 3 e-16 \quad 89.0$ ORF_comp2951_c3_seq2

gi|242051651|ref|XP_002454971.1| $31.80 \quad 217 \quad 128 \quad 8 \quad \begin{array}{lllll}121 & 750 & 26\end{array}$

229 4e-16 89.4 ORF_comp2951_c3_seq2 gi|870854054|gb|KMT05873.1|

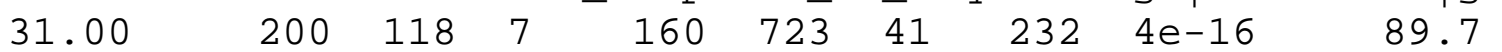

ORF_comp2951_c3_seq2 gi|313483704|gb|ADR51681.1| 30.46 $197 \quad 117$

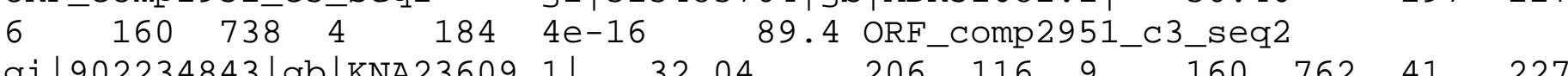

4e-16 89.7 ORF_comp2951_c3_seq2

gi|147719992|sp|Q2TE74.2|PCS2_LOTJA $\quad 31.0 \odot \quad 300 \quad 118 \quad 6 \quad 136 \quad 723$

$32 \quad 2154 \mathrm{e}-16 \quad 89.4$ ORF_comp2951_c3_seq2

gi|657981623|ref|XP_008382838.1| $32.84 \quad 204 \quad 117 \quad 7 \quad 154 \quad 753 \quad 29$

$2164 \mathrm{e}-16 \quad 89.4$ ORF_comp2951_c3_seq2 gi|902234842|gb|KNA23608.1|

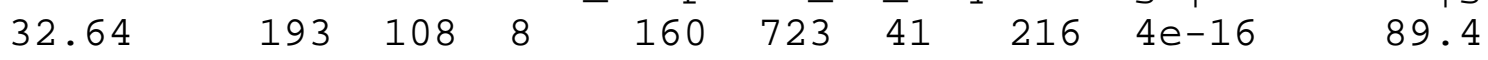

ORF_comp2951_c3_seq2 gi|922533447|ref|XP_013598130.1| 31.77

$1117 \quad 160 \quad 723 \quad 96 \quad 271 \quad 4 \mathrm{e}-16 \quad 89.7$ ORF_comp2951_c3_seq2

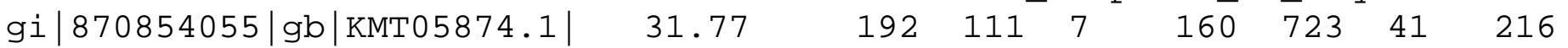


$4 e-16$

89.4 ORF_comp2951_c3_seq2

gi |923762135 |ref|XP_013677346.1|

31.77

1921117

$160723 \quad 96$

$2714 \mathrm{e}-16$

89.4

ORF_comp2951_c3_seq2 gi|870854053|gb|KMT05872.1| 31.77

192111

$7 \quad 160 \quad 723 \quad 16 \quad 191 \quad 4 \mathrm{e}-16 \quad 89.4$ ORF_comp2951_c3_seq2

gi|588263371|ref|XP_006960829.1| 30.73

247 5e-16 87.0 ORF_comp2951_c3_seq2

gi|731346810|ref|XP_010684651.1| 31.77

207 5e-16 89.4 ORF_comp2951_c3_seq2

29.891841123
ORF_comp2951_c3_seq2

$\begin{array}{llll}160 & 702 & 68 & 237 \\ 5 e-16\end{array}$

$\begin{array}{llllll}192 & 111 & 7 & 160 & 723 & 32\end{array}$ gi $|802733870| \mathrm{gb}|\mathrm{KKA} 73731.1|$

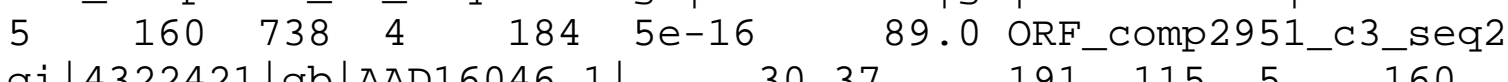

gi|313483741|gb|ADR51699.1| 29.74 $195 \quad 121$

gi|4322421|gb|AAD16046.1| $\quad 30.37 \quad 191 \quad 115 \quad 5 \quad \begin{array}{lllll}160 & 723 & 40 & 215\end{array}$

5e-16 89.0 ORF_comp2951_c3_seq2

29.21
ORF_comp2951_c3_seq2

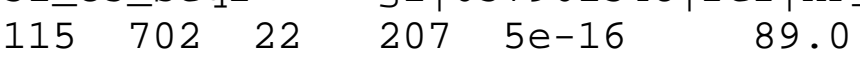

gi|657961548|ref|XP_008372367.1|

gi|685358931|ref|XP_009114083.1| 29.53

193

$\begin{array}{lllllll}116 & 5 & 115 & 678 & 50 & 227 & 5 \mathrm{e}-16\end{array}$

gi|870854052 |gb|KMT05871.1| 31.77

5e-16 89.0 ORF_comp2951_c3_seq2

30.89

$\begin{array}{llllll}191 & 114 & 5 & 160 & 723 & 40\end{array}$

89.4 ORF_comp2951_c3_seq2

$\begin{array}{lllllll}192 & 111 & 7 & 160 & 723 & 41 & 216\end{array}$

gi | 297794999|ref|XP_002865384.1|

ORF_comp2951_c3_seq2

gi|29470177|gb|AA074500.1| 32.34

201116

$7 \quad 160750 \quad 40 \quad 2245 \mathrm{e}-16 \quad 89.0$ ORF_comp2951_c3_seq2

gi|985450624|ref|XP_015386247.1| 30.21

179 5e-16 89.0 ORF_comp2951_c3_seq2

gi|823189101|ref|XP_012490722.1| 32.29

$1796 e-16 \quad 89.0$ ORF_comp2951_c3_seq2

gi|823189093|ref|XP_012490720.1| 32.29

217 6e-16 89.0 ORF_comp2951_c3_seq2

$\begin{array}{lll}31.80 & 217 & 128 \quad 8 \\ \text { ORF Comp2951 c3 seq2 }\end{array}$

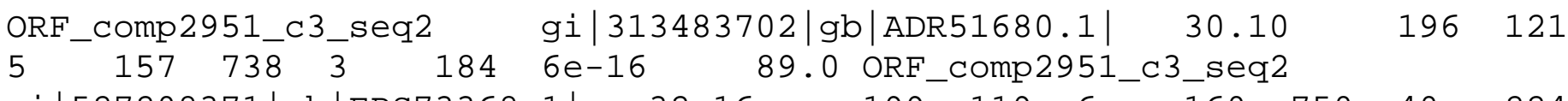

$192114 \quad 6 \quad 160 \quad 723 \quad 4$

$\begin{array}{llllll}192 & 110 & 7 & 160 & 723 & 4\end{array}$

$\begin{array}{llllll}192 & 110 & 7 & 160 & 723 & 42\end{array}$ gi | 992271392 | gb |KXG31593 . 1 |

$121750 \quad 26 \quad 2296 \mathrm{e}-16 \quad 89.0$

gi|527208371|gb|EPS73368.1| $32.16 \quad 199 \quad 119 \quad 6 \quad \begin{array}{lllll}160 & 750 & 40 & 224\end{array}$

6e-16 89.0 ORF_comp2951_c3_seq2

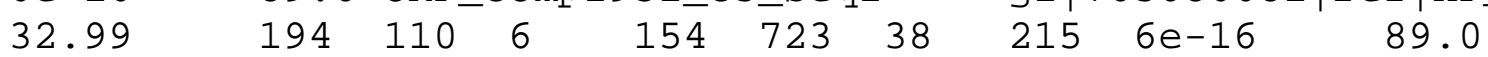

ORF_comp2951_c3_seq2 gi|909606181|gb|KNE96553.1| 31.84

$5 \quad 154714 \quad 118 \quad 304$ 6e-16 89.0 ORF_comp2951_c3_seq2

gi|284466089|gb|ACL00594.3| $30.89 \quad 191 \quad 114 \quad 5 \quad 160 \quad 723 \quad 40 \quad 215$

6e-16 89.0 ORF_comp2951_c3_seq2 gi|731346807|ref|XP_010684650.1|

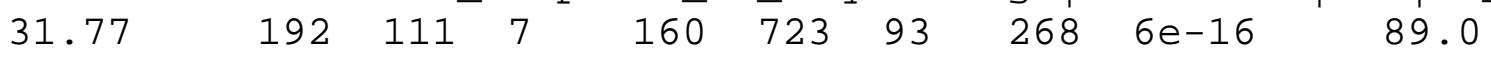

ORF_comp2951_c3_seq2 gi|731346803|ref|XP_010684648.1| 31.77

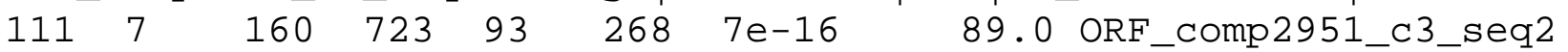

gi|313483755|gb|ADR51706.1| $30.46 \quad \begin{array}{lllllll}197 & 117 & 6 & 160 & 738 & 4 & 184\end{array}$

7e-16 88.6 ORF_comp2951_c3_seq2 gi|823127937|ref|XP_012440935.1|

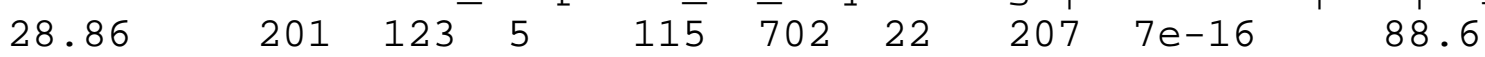

ORF_comp2951_c3_seq2 gi|731346805|ref|XP_010684649.1| 31.77

$1117 \quad 160 \quad 723 \quad 93 \quad 268 \quad 7$ e-16 89.0 ORF_comp2951_c3_seq2

gi|313483709|gb|ADR51683.1| 30.46

7e-16 88.6 ORF_comp2951_c3_seq2

$30.46 \quad 197 \quad 117^{-} 6 \quad 160 \quad 738 \quad 4 \quad 184 \quad 7 \mathrm{e}-16 \quad 88.6$

ORF_comp2951_c3_seq2 gi|823127935|ref|XP_012440927.1| 28.86

$1235115 \quad 702 \quad 22 \quad 207 \quad 8$ e-16 88.6 ORF_comp2951_c3_seq2

gi|823127931|ref|XP_012440911.1| $28.86 \quad 201 \quad 123 \quad 5 \quad \begin{array}{llll}115 & 702 & 22\end{array}$

207 8e-16 88.6 ORF_comp2951_c3_seq2

gi|551561391|ref|XP_005767549.1| 33.50

228 8e-16 85.5 ORF_comp2951_c3_seq2

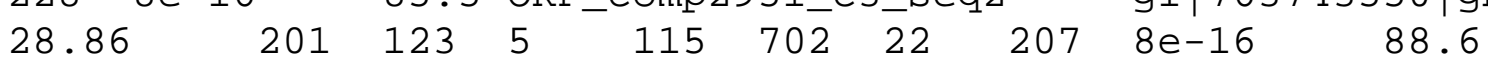

ORF_comp2951_c3_seq2 gi|313483699|gb|ADR51679.1| $29.95 \quad 197 \quad 118$

$6 \quad 160738 \quad 4 \quad 184$ 9e-16 88.2 ORF_comp2951_c3_seq2

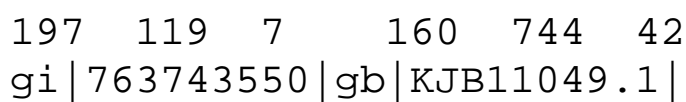

$8 \mathrm{e}-16 \quad 88.6$ 
gi|470240016|ref|XP_004351977.1| 28.80

242 9e-16 88.2 ORF_comp2951_c3_seq2

. 192110

ORF_comp2951_c3_seq2

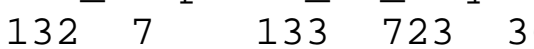
$\begin{array}{llll}160 & 723 & 40 & 215\end{array}$ gi|915844541|ref|WP_050904055.1| 29.03 $\begin{array}{llllll}191 & 119 & 6 & 160 & 723 & 66\end{array}$ gi|53760453|gb|AAU93349.1| $9 \mathrm{e}-16 \quad 88.6$ $2509 \mathrm{e}-16$ 85.5 ORF_comp2951_c3_seq2 gi|672183478|ref|XP_008812019.1| 31.86 224 9e-16 88.6 ORF_comp2951_c3_seq2 32.81 $\begin{array}{lcc}32.81 & 1921097 \\ \text { ORF_comp2951_c3_seq2 }\end{array}$ $\begin{array}{llll}160 & 723 & 40 & 215\end{array}$ $\begin{array}{llllll}204 & 117 & 6 & 160 & 762 & 40\end{array}$ gi|966806441|gb|ALT55649.1| gi|449445620|reflXP 004140570.11

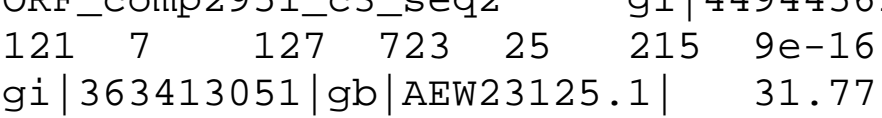
9e-16 88.2 ORF_comp2951_c3_seq2 $\begin{array}{lll}32.28 & 189114 \quad 4 \\ \text { ORF_comp2951_c3_seq2 }\end{array}$ $\begin{array}{llllll}7 & 160 & 723 & 40 & 215 & 1 \mathrm{e}-15\end{array}$ $160 \quad 723 \quad 40$ (XP_.04140570.1| 29.95 207 gi|823127933|ref|XP_012440917.1| 28.86 207 1e-15 88.2 ORF_comp2951_c3_seq2 gi|743862902|ref|XP_010943664.1| 33.17 224 1e-15 88.2 ORF_comp2951_c3_seq2 $\begin{array}{lllllll}28.86 & 201 & 123 & 5 & 115 & 702 & 22\end{array}$ 88.2 ORF_comp2951_c3_seq2 $\begin{array}{llllll}192 & 111 & 7 & 160 & 723 & 40\end{array}$ 215 gi 674920164 | emb |CDY13128.1| $215 \quad 9 \mathrm{e}-16 \quad 88.2$ 88.2
32.29

192110

ORF_comp2951_c3_seq2 gi | 46949222 | gb|AAT07467.1| $\begin{array}{llllll}205 & 113 & 7 & 160 & 762 & 40\end{array}$ gi $|763743549| \mathrm{gb}|\mathrm{KJB} 11048.1|$ $7 \quad 160 \quad 723 \quad 40 \quad 215 \quad 1 \mathrm{e}-15$ gi|926792009|ref|XP_013904478.1| 30.16 198 1e-15 87.8 ORF_comp2951_c3_seq2 $30.89 \quad 191 \quad 114 \quad 5$
ORF_comp2951_c3_seq2 $\begin{array}{lllll}160 & 723 & 4 & 179 & 1 \mathrm{e}-15\end{array}$ 88.2 31.77 192111 $201123{ }^{2} \quad 115 \quad 702 \quad 22$

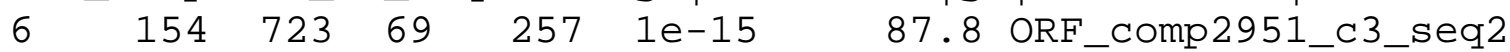
gi|645260011|ref|XP_008235633.1| $31.86 \quad 204 \quad 119 \quad 7 \quad 154 \quad 753 \quad 38$ 225 1e-15 88.2 ORF_comp2951_c3_seq2 $\begin{array}{llllllllll}31.09 & 193 & 115 & 5 & 154 & 723 & 40 & 217 & 1 \mathrm{e}-15 & 88.2\end{array}$ ORF_comp2951_c3_seq2

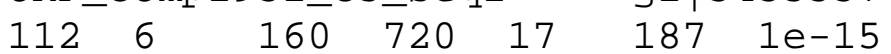
gi $|122208907|$ sp |Q2TSC7 .1|PCS1_LOTJA gi $|545355774|$ ref |XP_005643662.1| 30.16 $160 \quad 720 \quad 24$ gi|947119173|gb|KRH67422.1| 1e-15 87.8 $38 \quad 215$ 1e-15 88.2 ORF_comp2951_c3_seq2 gi|384491545|gb|EIE82741.1| 30.99 1e-15 87.4 ORF_comp2951_c3_seq2 $\begin{array}{lllllll}213 & 98 & 8 & 115 & 732 & 53 & 223\end{array}$ gi|590715531|ref|XP_007050222.1| 30.20 $202 \quad 1196$ $115702 \quad 22$ $207 \quad 1 e-15$ 87.8 ORF_comp2951_c3_seq2 gi|922548834|ref|XP_013602398.1| 31.77 $\begin{array}{lllllllll}111 & 7 & 160 & 723 & 40 & 215 & 1 \mathrm{e}-15 & 87.8 & \text { ORF_comp2951_c3_seq2 }\end{array}$ $\begin{array}{llllllll}\text { gi }|313483719| \mathrm{gb} \mid \text { ADR51688.1| } & 30.46 & 197 & 117 & 6 & 160 & 738 & 4\end{array}$ 1e-15 87.8 ORF_comp2951_c3_seq2 gi|220683835|gb|ACL80669.1| $\begin{array}{llllllllll}32.29 & 192 & 110 & 7 & 160 & 723 & 40 & 215 & 1 \mathrm{e}-15 & 87.8\end{array}$ ORF_comp2951_c3_seq2 gi|922346013|ref|XP_013449921.1| 31.15

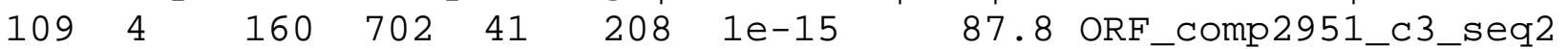
gi|255964729|gb|ACU44656.1| 30.73 $\begin{array}{llllll}192 & 113 & 7 & 160 & 723 & 40\end{array}$ $1 \mathrm{e}-15$ 87.8 
Horizontal gene transfer of phytochelatin synthases from bacteria to extremophilic green algae; Microbial Ecology; Olsson S*, Penacho V, Puente-Sánchez F, Díaz S, Aguilera A; * corresponding author, Department of Agricultural Sciences, P.O. Box 27, 00014 University of Helsinki, Finland, sanna.olsson@helsinki.fi

Online resource 5 Introns in the predicted PCS genes from C. acidophila and D. acidophila. There are three spliceosomal introns in CaPCS2, four in CaPCS1 and three in DaPCS1. The introns are either the most common GT-AG splice site or variations of it.

\begin{tabular}{|l|l|l|}
\hline Gene & Intron 5' end & Intron 3' end \\
\hline CaPCS2 & GAG | GTGAGG & TAG | G \\
\hline & CAG | GTGTGT & TAN | G \\
\hline & CAA | GTAAGG & TAG | G \\
\hline CaPCS1 & AAG | GTAAGC & CAG | G \\
\hline & CAG | GTACTT & CAG | C \\
\hline & CAG | GTGATG & CAG | A \\
\hline DaPCS1 & CAG | GTGAGA & TAG | G \\
\hline & CTG | GTGGGT & GAG | G \\
\hline & CAG | GCGAGC & CAG | A \\
& CAA | GTGCGG & CAG | G \\
\hline
\end{tabular}


Horizontal gene transfer of phytochelatin synthases from bacteria to extremophilic green algae; Microbial Ecology;

olsson S*, Penacho V, Puente-Sánchez F, Díaz S, Aguilera A;

* corresponding author, Department of Agricultural Sciences, P.0. Box 27, 00014 University of Helsinki, Finland, sanna.olsson@helsinki.fi.

Online Resource 6 Isoforms of component comp17619_c $\odot$ coding for the CaPCS1 gene in Chlamydomonas acidophila

>comp17619_c0_seq1

GCTCAATGTTAGAACCGCAAAATTTTGCTGTGGCTACCTTAAAAGTTTTTCAGGTCTAAAATCCAGTAAGTTCA TGTCCA

AGCTGTGCATACCAAACTGCTTATGTATCACAGTCTTATCCCCATGTCCAGGTGCTATCACACCCTCCAATTTT GAGAAA

CGACACGCAACAATATCTTTTTCGGGCACTCCTTCTTCCTTGAAGGGAATTGAATACAACAGCGCCACATTGTC TCCAAT

GAATGAAACAGTTCCTGAGATGACATGCAGCACTCAAAAATCAAGCTGTACCTCTAGGGTTGAGCATGTTTGTG GAGAGC

CACССTCTTCAAAAAATCCTATCTCAATACACCTGTATAATATCTCTGGTGCCGGGTCTGTGCAACCTTCTCAA TCAACA

GCCACTCCTATTGTGGGGGTGACAGCTCTTCAAACAGGCTCTGGGTTTAGAAGGACATTTTATAAGCGTCATCT ACCAAG

CCCACCAGCAATTGCTTTTTCCTCACCTCAAGGGCGTCAAATATTTCAAGAGGCGCTAATGGGCGGCACCCTAG GAGGCT

TTTTCAAGTTGATGGAGCAATTCAGCATGCAAGACGAACCAGCCTTTTGTGGTCTTACAAGCTTGACTATGGTG CTGAAT

GCACTCTCCATCGACCCGCGGCGAACATGGAAGGTGCATGGCGTTGGTTCCATGAGGCCATGCTAGACTGCTGC AGGTGA

TGAGTCACCAAATCAAACGCTGTGATTCCTCAGCTCCTGATGCTCACTTTTCAACTACATCTTAATCCCATCCC CATCCA

TCGCAACAGCTTATGACCCTCATATCAACTAGAAGTGATATGGAAACTTTGCTTTCTCTCTCACAGACCATTGG AATCTG

TTAAGGAGGATGGCATAACCCTTGCTCAGGCTGCATGTCTTGCCCGCTGCAACGGGGCCCGCGTTGACATGATG CGACAT

GGCACCTTCTCAGAGGCTGAGTTCAGGCAGCAAGTGATGGATGTGTGTAGGAGTGGGGAGGAGCATCTGGTGGT TAGCTA

CGCCCGACGTGCTTTTAGTCAGACTGGGGATGGTCATTTTAGTCCTGTTGGAGGATACCATGAGGGCCAGGACT TGGTAC

TAATCTTGGATGTGGCCCGCTTCAAGTATCCTCCACACTGGGTACCTCTACCTCTGCTCTATGAGGCCATGTCA CATGTA

GACCCCACCACTGGCCTCCCACGTGGCTACATGCTCCTGTCTGCTCACCCGCTGCTGGACTCCGCCATGTTCTC TCTAGA

CATCCGTCAAGAGGGATGGAAGCTGGCAAGAAGCTATGTCACCATGATGCCTGTAGTCTTGGAGCAGTACATGT CCTTGA

GAGCTCAGAGGCTGCAGCAGGAGCAGCAGATGTTGGCAGCCAAGGAAGACAAGCTTGTGGTTATGGAGCATGCT GTAAGG

CATTTAGTCCTAGGTATTCCAAAACAAGGCATTATATCCTTCATTGCCATTCGGGAGCTGGCAAGAGCGCCTGG AGAGAA

GTGTGTGCCTCAGAAGTCTAGAGAGACTCTATTGTTGGAGCTGAGGGCCATGGTTTGTATCAGCTGGTGCTCAA GGCAAT

CAAAGGGGATGGACCATCTGCCTCGGCAGCAGCTGATAGAGAAGCTGGTATGCGGAGCCCTGAGGATACAGAGG ACATGG

GCGGATCTCTGTGTTCTTCCTCGGCCTACATGGCAGATCGTTGTACACTTCTCATCATGCTGCAGCCCCCCTCA GCATGG

CCAGCCTGGTCATCTCCAGAGCTCAACACTCAGTGGCAAGCTCTTTTAGATCTAACGAATTTCAGTGTTGTTGC TACTGA

GGTGGCTTACATGCGCCAGCAGTTTGTACATGTGGATGAAGTGATGCAGGGAGACTCAGTGGACGCTACTTGTG 
GCCAAC

CTGCATGCAAATCCAGACTCGACTGTGCAGTTCTGGGACATTGACTTTGAATGACTGCTTCATGCTGAGAACAA CTGTGA

TTCTGTTGGCATGACTGAGCCAATCAATATCTCTTTTGATAAACCAGACTGAATATACATTATGGGTTTGAGCA AATGCC

CATAAGAGACTTTGTGCACGTCCAACATCCTTAGTCACTGTGACTAATGAGACTTTAAACCGCTCTCCTTCGCA CATAG

>comp17619_c0_seq2

GCTCAATGTTAGAACCGCAAAATTTTGCTGTGGCTACCTTAAAAGTTTTTCAGGTCTAAAATCCAGTGCTATCA CACCCT

CCAATTTTGAGAAACGACACGCAACAATATCTTTTTCGGGCACTCCTTCTTCCTTGAAGGGAATTGAATACAAC AGCGCC

ACATTGTCTCCAATGAATGAAACAGTTCCTGAGATGACATGCAGCACTCAAAAATCAAGCTGTACCTCTAGGGT TGAGCA

TGTTTGTGGAGAGCCACCCTCTTCAAAAAATCCTATCTCAATACACCTGTATAATATCTCTGGTGCCGGGTCTG TGCAAC

CTTCTCAATCAACAGCCACTCCTATTGTGGGGGTGACAGCTCTTCAAACAGGCTCTGGGTTTAGAAGGACATTT

TATAAG

CGTCATCTACCAAGCCCACCAGCAATTGCTTTTTCCTCACCTCAAGGGCGTCAAATATTTCAAGAGGCGCTAAT GGGCGG

CACCCTAGGAGGCTTTTTCAAGTTGATGGAGCAATTCAGCATGCAAGACGAACCAGCCTTTTGTGGTCTTACAA GCTTGA

CTATGGTGCTGAATGCACTCTCCATCGACCCGCGGCGAACATGGAAGGTGCATGGCGTTGGTTCCATGAGGCCA TGCTAG

ACTGCTGCAGGTGATGAGTCACCAAATCAAACGCTGTGATTCCTCAGCTCCTGATGCTCACTTTTCAACTACAT CTTAAT

CCCATCCCCATCCATCGCAACAGCTTATGACCCTCATATCAACTAGAAGTGATATGGAAACTTTGCTTTCTCTC TCACAG

ACCATTGGAATCTGTTAAGGAGGATGGCATAACCCTTGCTCAGGCTGCATGTCTTGCCCGCTGCAACGGGGCCC GCGTTG

ACATGATGCGACATGGCACCTTCTCAGAGGCTGAGTTCAGGCAGCAAGTGATGGATGTGTGTAGGAGTGGGGAG

GAGCAT

CTGGTGGTTAGCTACGCCCGACGTGCTTTTAGTCAGACTGGGGATGGTCATTTTAGTCCTGTTGGAGGATACCA TGAGGG

CCAGGACTTGGTACTAATCTTGGATGTGGCCCGCTTCAAGTATCCTCCACACTGGGTACCTCTACCTCTGCTCT ATGAGG

CCATGTCACATGTAGACCCCACCACTGGCCTCCCACGTGGCTACATGCTCCTGTCTGCTCACCCGCTGCTGGAC TCCGCC

ATGTTCTCTCTAGACATCCGTCAAGAGGGATGGAAGCTGGCAAGAAGCTATGTCACCATGATGCCTGTAGTCTT GGAGCA

GTACATGTCCTTGAGAGCTCAGAGGCTGCAGCAGGAGCAGCAGATGTTGGCAGCCAAGGAAGACAAGCTTGTGG TTATGG

AGCATGCTGTAAGgCATTTAGTCCTAGgTATTCCAAAACAAGGCATTATATCCTTCATTGCCATTCGGGAGCTG GCAAGA

GCGCCTGGAGAGAAGTGTGTGCCTCAGAAGTCTAGAGAGACTCTATTGTTGGAGCTGAGGGCCATGGTTTGTAT CAGCTG

GTGCTCAAGGCAATCAAAGGGGATGGACCATCTGCCTCGGCAGCAGCTGATAGAGAAGCTGGTATGCGGAGCCC TGAGGA

TACAGAGGACATGGGCGGATCTCTGTGTTCTTCCTCGGCCTACATGGCAGATCGTTGTACACTTCTCATCATGC TGCAGC

CCCCCTCAGCATGGCCAGCCTGGTCATCTCCAGAGCTCAACACTCAGTGGCAAGCTCTTTTAGATCTAACGAAT TTCAGT

GTTGTTGCTACTGAGGTGGCTTACATGCGCCAGCAGTTTGTACATGTGGATGAAGTGATGCAGGGAGACTCAGT GGACGC

TACTTGTGGCCAACCTGCATGCAAATCCAGACTCGACTGTGCAGTTCTGGGACATTGACTTTGAATGACTGCTT CATGCT 
GAGAACAACTGTGATTCTGTTGGCATGACTGAGCCAATCAATATCTCTTTTGATAAACCAGACTGAATATACAT

TATGGG

TTTGAGCAAATGCCCATAAGAGACTTTGTGCACGTCCAACATCCTTAGTCACTGTGACTAATGAGACTTTAAAC CGCTCT CCTTCGCACATAG

>comp17619_c0_seq3

GCTCAATGTTAGAACCGCAAAATTTTGCTGTGGCTACCTTAAAAGTTTTTCAGGTCTAAAATCCAGTAAGTTCA TGTCCA

AGCTGTGCATACCAAACTGCTTATGTATCACAGTCTTATCCCCATGTCCAGGTGCTATCACACCCTCCAATTTT GAGAAA

CGACACGCAACAATATCTTTTTCGGGCACTCCTTCTTCCTTGAAGGGAATTGAATACAACAGCGCCACATTGTC TCCAAT

GAATGAAACAGTTCCTGAGATGACATGCAGCACTCAAAAATCAAGCTGTACCTCTAGGGTTGAGCATGTTTGTG GAGAGC

CACССТСTTCAAAAAATCCTATCTCAATACACCTGTATAATATCTCTGGTGCCGGGTCTGTGCAACCTTCTCAA TCAACA

GCCACTCCTATTGTGGGGGTGACAGCTCTTCAAACAGGCTCTGGGTTTAGAAGGACATTTTATAAGCGTCATCT ACCAAG

CCCACCAGCAATTGCTTTTTCCTCACCTCAAGGGCGTCAAATATTTCAAGAGGCGCTAATGGGCGGCACCCTAG GAGGCT

TTTTCAAGTTGATGGAGCAATTCAGCATGCAAGACGAACCAGCCTTTTGTGGTCTTACAAGCTTGACTATGGTG CTGAAT

GCACTCTCCATCGACCCGCGGCGAACATGGAAGGTGCATGGCGTTGGTTCCATGAGGCCATGCTAGACTGCTGC AGACCA

TTGGAATCTGTCAAGGAGGATGGCATAACCCTTGCTCAGGCTGCATGTCTTGCCCGCTGCAACGGGGCCCGCGT TGACAT

GATGCGACATGGCACCTTCTCAGAGGCTGAGTTCAGGCAGCAAGTGATGGATGTGTGTAGGAGTGGGGAGGAGC ATCTGG

TGGTTAGCTACGCCCGACGTGCTTTTAGTCAGACTGGGGATGGTCATTTTAGTCCTGTTGGAGGATACCATGAG GGCCAG

GACTTGGTACTAATCTTGGATGTGGCCCGCTTCAAGTATCCTCCACACTGGGTACCTCTACCTCTGCTCTATGA GGCCAT

GTCACATGTAGACCCCACCACTGGCCTCCCACGTGGCTACATGCTCCTGTCTGCTCACCCGCTGCTGGACTCCG CCATGT

TCTCTCTAGACATCCGTCAAGAGGGATGGAAGCTGGCAAGAAGCTATGTCACCATGATGCCTGTAGTCTTGGAG CAGTAC

ATGTCCTTGAGAGCTCAGAGGCTGCAGCAGGAGCAGCAGATGTTGGCAGCCAAGGAAGACAAGCTTGTGGTTAT GGAGCA

TGCTGTAAGGCATTTAGTCCTAGgTATTCCAAAACAAGGCATTATATCCTTCATTGCCATTCGGgAGCTGGCAA GAGCGC

CTGGAGAGAAGTGTGTGCCTCAGAAGTCTAGAGAGACTCTATTGTTGGAGCTGAGGGCCATGGTTTGTATCAGC TGGTGC

TCAAGGCAATCAAAGGGGATGGACCATCTGCCTCGGCAGCAGCTGATAGAGAAGCTGGTATGCGGAGCCCTGAG GATACA

GAGGACATGGGCGGATCTCTGTGTTCTTCCTCGGCCTACATGGCAGATCGTTGTACACTTCTCATCATGCTGCA GCCCCC

CTCAGCATGGCCAGCCTGGTCATCTCCAGAGCTCAACACTCAGTGGCAAGCTCTTTTAGATCTAACGAATTTCA GTGTTG

TTGCTACTGAGGTGGCTTACATGCGCCAGCAGTTTGTACATGTGGATGAAGTGATGCAGGGAGACTCAGTGGAC GCTACT

TGTGGCCAACCTGCATGCAAATCCAGACTCGACTGTGCAGTTCTGGGACATTGACTTTGAATGACTGCTTCATG CTGAGA

ACAACTGTGATTCTGTTGGCATGACTGAGCCAATCAATATCTCTTTTGATAAACCAGACTGAATATACATTATG GGTTTG

AGCAAATGCCCATAAGAGACTTTGTGCACGTCCAACATCCTTAGTCACTGTGACTAATGAGACTTTAAACCGCT CTCCTT CGCACATAG 
>comp17619_c0_seq4

CCGCTCTCCTTCGCACATAGGGACACGCCTCCCAGGAAGGTTTGCACACATCATATCCTCTAACAACATATTTT CTTTCA

TTCTTATTTACATGACAAATATAACACATAGTCATCAAAAATTGAGGGATCGCAATCAGAGGCCTTCACTTATA TTTTCA

TTTTATTCATTACAGATCTCAACACTTGgTGCGCATTTTGGTCTCAGTGTTATTTTAATCACATTACTCCCAAA ACATAT

TTCTTAAACATGGCCTTCAAGAACCTTGCCAGACCTAGGACAATGACACACATTTCACAACGCCCTAAGCAGTC GTTGTA

AACAATACTCTTATTACTCTTACTCTCAACGGGCTCAAGCCATCCTGCAGTAGGCTAATAAAAGATAATCCATC TAACAC

ATTGAGAATATTACACCATTTCATTGCAGAGACGGACCTGCCTAGACTTCTGCAAGCTGGTGATGTCCAGAAAG AGCTTT

TATCCTTCCTGATGAAGGCGGCACAAAACAACCGCAACAGGTTCACGAAGAAGTAGTTAGCCCTGCTGCTTATG CAGCCT

GTTACTCATTTCTGCGATCTTTTTCATATCTTCGGCTTCTTCCTGCAACTTGGCCCACACACCACTATCTCCCA TGCGCC

ACATCCAATTATTCTCAGCTCGCCCAGGAGTATTCATTCTTGCAGTATTATCTAATCTCATCACATCCTGCATC ATGACA

ATGCATGTGGCTGCCACGCTGTTCATGCAAGCTCGCAGCAGGTCCCAAGCAATATCATCTCCAGTAGTTTGTAG GTATTG

ACACAGGTACTCCTTGTCTTCCTTGGAAGCGCTGTCTTTAAACCAGCCCACCGATGTCTCATTGTCGTGGGTGC CAGGAT

ACACAAAACAATTAGCATAGTGGTTGTGAGGGAGGTGGACATTGCCTGGGCCACCTCCCCATGCAAACTGGAGC ACCACC

ATGCCAGGAGCACCAATACTCTCCCTCAACTCCACCACGTCAGTGGTGATGACCCCTAGATCCTCCGCCAAGAT CGGCAC

TGCCCCTAGCTTGGCCTTGATAGCTGTGAACAGCTCCAATCCGGGCCCTTTACACCACGACCCCCCCATGGCAG TCTCAG

CTTTACCATCAACAGCCCAATAGCCTGCAAAGCCCCGGAAGTGATCAATCCTTGTTTGGTCATAAAGCTGCATA GCTCGC

CCCATGCGTTGACACCACCAGTCGTAGTTCTGCGCCTTGTGAGCCGACCACTTGTAAAGCGGACTCCCCCACAA CTGACC

AGTCTCTGAGAATGCATCAGGTGGCACCCCACTGACATTAGCTGGCAGGCCTGTAGGTCCTAGCTCAAAGAGGT

CTTGAT

TAGACCACACATCTGCTGAGTGCCCACCCACATAAATGGGCATGTCTCCAATGATGTCCACACCTTTTGAGTTT GCATAC

GCTCTGATAGCCTTCCACTGACGATCAAAGAAGAACTGTATGGCAATAAATTCCTCAATCGCCTGCTTATGTTC CTCCTG

GAATGTTTTCAGAGCATCCGgGTCCCTGAAGCGCAGCGACTCTGGCCAATCCCACCAGGCCATCGTAGATAGTT CTGAAG

GTTCCTTGCTACATCAAACACAGCGCTTTCCTCAATCCATGGATTCAATTTCCTAAATGCTATCATCTCCTCCC TGAGAT

CCTTAAATTTGATGTCTTCTAGGAGCCTTTCAGCTGCAATGCGCAAGGCAGGCGTTTTAGCTGCCATGACAGCA GGGTAG

TCAATGTTGCCAACTGAGACTTTAGCAGGGGCGTCCTTGGCATGTATCAAGCCCTCTTGAATCAACTCATCAAT AGAGAT

GATGAGTGGATTGCCACAATTTGCGTCAGTTCCACTGTAAGGAGAGTAAAACATGGGGTCTGGAGGCCCAAGTG GTAGCA

TCTGCCAGCAAGTCATCCCTGCTGTAACAAGCCAGTCTACAAACAGTCTTGCCTCTCCCCCAACTCGCCCACAC CATAAG

GTCCTGGTAGAGAAGTTGGATGCAAAATAATCCCAGCCCTCCGTTTAATTGCATTTTTGGGTTGTAAAACTGTC ATATGC

TTGCGGTAGTGTATCCCCTATTGAGGCTTTCTCATAAACTGATGACATGTTTAATGCAGGTGCCTGGGCCCACG GCAGTG

CCCAAGTCTGTACTTTCATCTTTGATGTTGAAATTTTGGCATCACGATGCATGGAGACAGCACACGATTTCCTA CGTATA 
TCATGCGCTAACAAACACGATCTAGCTTTGATCTGTTCTGATTTGATGAAGAGTTTCGACATCAGAATATCTAG TGAACA

AACACAATATCTATGTTCTGATCACACAGAAATTATCAATGTATATTATTATCTTTAGTCTATTCAAGCATCGT TAAG

>comp17619_c0_seq5

GCTCAATGTTAGAACCGCAAAATTTTGCTGTGGCTACCTTAAAAGTTTTTCAGGTCTAAAATCCAGTGCTATCA CACCCT

CCAATTTTGAGAAACGACACGCAACAATATCTTTTTCGGGCACTCCTTCTTCCTTGAAGGGAATTGAATACAAC AGCGCC

ACATTGTCTCCAATGAATGAAACAGTTCCTGAGATGACATGCAGCACTCAAAAATCAAGCTGTACCTCTAGGGT TGAGCA

TGTTTGTGGAGAGCCACCCTCTTCAAAAAATCCTATCTCAATACACCTGTATAATATCTCTGGTGCCGGGTCTG TGCAAC

CTTCTCAATCAACAGCCACTCCTATTGTGGGGGTGACAGCTCTTCAAACAGGCTCTGGGTTTAGAAGGACATTT TATAAG

CGTCATCTACCAAGCCCACCAGCAATTGCTTTTTCCTCACCTCAAGGGCGTCAAATATTTCAAGAGGCGCTAAT GGGCGG

CACCCTAGGAGGCTTTTTCAAGTTGATGGAGCAATTCAGCATGCAAGACGAACCAGCCTTTTGTGGTCTTACAA GCTTGA

CTATGGTGCTGAATGCACTCTCCATCGACCCGCGGCGAACATGGAAGGTGCATGGCGTTGGTTCCATGAGGCCA TGCTAG

ACTGCTGCAGACCATTGGAATCTGTCAAGGAGGATGGCATAACCCTTGCTCAGGCTGCATGTCTTGCCCGCTGC AACGGG

GCCCGCGTTGACATGATGCGACATGGCACCTTCTCAGAGGCTGAGTTCAGGCAGCAAGTGATGGATGTGTGTAG GAGTGG

GGAGGAGCATCTGGTGGTTAGCTACGCCCGACGTGCTTTTAGTCAGACTGGGGATGGTCATTTTAGTCCTGTTG GAGGAT

ACCATGAGGGCCAGGACTTGGTACTAATCTTGGATGTGGCCCGCTTCAAGTATCCTCCACACTGGGTACCTCTA CCTCTG

CTCTATGAGGCCATGTCACATGTAGACCCCACCACTGGCCTCCCACGTGGCTACATGCTCCTGTCTGCTCACCC GCTGCT

GGACTCCGCCATGTTCTCTCTAGACATCCGTCAAGAGGGATGGAAGCTGGCAAGAAGCTATGTCACCATGATGC CTGTAG

TCTTGGAGCAGTACATGTCCTTGAGAGCTCAGAGGCTGCAGCAGGAGCAGCAGATGTTGGCAGCCAAGGAAGAC AAGCTT

GTGGTTATGGAGCATGCTGTAAGGCATTTAGTCCTAGGTATTCCAAAACAAGGCATTATATCCTTCATTGCCAT TCGGGA

GCTGGCAAGAGCGCCTGGAGAGAAGTGTGTGCCTCAGAAGTCTAGAGAGACTCTATTGTTGGAGCTGAGGGCCA TGGTTT

GTATCAGCTGGTGCTCAAGGCAATCAAAGGGGATGGACCATCTGCCTCGGCAGCAGCTGATAGAGAAGCTGGTA TGCGGA

GCCCTGAGGATACAGAGGACATGGGCGGATCTCTGTGTTCTTCCTCGGCCTACATGGCAGATCGTTGTACACTT CTCATC

ATGCTGCAGCCCCCCTCAGCATGGCCAGCCTGGTCATCTCCAGAGCTCAACACTCAGTGGCAAGCTCTTTTAGA TCTAAC

GAATTTCAGTGTTGTTGCTACTGAGGTGGCTTACATGCGCCAGCAGTTTGTACATGTGGATGAAGTGATGCAGG GAGACT

CAGTGGACGCTACTTGTGGCCAACCTGCATGCAAATCCAGACTCGACTGTGCAGTTCTGGGACATTGACTTTGA ATGACT

GCTTCATGCTGAGAACAACTGTGATTCTGTTGGCATGACTGAGCCAATCAATATCTCTTTTGATAAACCAGACT GAATAT

ACATTATGGGTTTGAGCAAATGCCCATAAGAGACTTTGTGCACGTCCAACATCCTTAGTCACTGTGACTAATGA GACTTT AAACCGCTCTCCTTCGCACATAG

>comp17619_c0_seq6

CCGCTCTCCTTCGCACATAGGGACACGCCTCCCAGGAAGGTTTGCACACATCATATCCTCTAACAACATATTTT 
CTTTCA

TTCTTATTTACATGACAAATATAACACATAGTCATCAAAAATTGAGGGATCGCAATCAGAGgCCTTCACTTATA TTTTCA

TTTTATTCATTACAAGATCTCAACACTTGGTTCGCATTTTGGTCTCAGTGTTATTTTAATCACATTACTCCTAA AACATA

TTTCATAAACATGGCCTTCAAGAAACTTGCCAGACCAAGGATGACAATGACAATCATTTCACAACGCCCTAAGC AGTCAT

TTCACGACGCTCTAAGCAGTCGTTGTAAACTTGTAAACAATACGGCTCTTATTAGTCTTACTCTCAACGGGCTT AAGCCA TCCTGCAGTAGGCTAATAAA 
Horizontal gene transfer of phytochelatin synthases from bacteria to extremophilic green algae; Microbial Ecology;

olsson S*, Penacho V, Puente-Sánchez F, Díaz S, Aguilera A;

* corresponding author, Department of Agricultural Sciences, P.0. Box 27, 00014 University of Helsinki, Finland, sanna.olsson@helsinki.fi.

Online Resource 7 Isoforms of component comp11852_c $\odot$ coding for the CaPCS2 gene in Chlamydomonas acidophila

$>$ comp11852_c0_seq1_len=2482

TTTGTTCACTTTTCGGCAGTAAATAAGCAATATTGTAGTCAAGTTGTTATCCTCGCTCTTACCTTTTGATTTCA AGGATG

CTTTCAGTCTTGCTTTGTACCAGAATTTTGAAAACATACATTCTCTTTCAAGTTCTGACCTCATCCGTCCAAGG GGAGTT

GGGTCTGCCTTGTTCCCTTTTGTCATTAACATCACACGAAGGACAAAAGGCCTTCATTCGTGCCAAGTATAAGG GCGATG

CATTTTCCATGCTGGAGAACTTTGTTGCACAGGTCAACGATGCGTACTGCGGGCCAGCCTCTGCTGTAACAGTC CTGAAT

TCTTTGGGGTGGGGCTCCCTATGCAGGACCCTGCTTCAGGGTTAGACTTGCCTGAATTTGCATATTACACACAA GACAAC

GTATTCTCACTAGAAACAGAAAGAGTGAAGCCCAAAGCTCAGATCAGGGAAGAGGGCCTCAGCCTATCAGAGCT CTCAAG

TTTCATCGCTGCTCACTCAGGAGTCAACGCCACGTACCGCCACAGCAGGGACATGAAAGACATGAATGAGTTTC GACAGC

TGGCTGTGTCAGCTCTAGCACAGCCTAATGTGTATGTTATTGTCAACTTCTTCCGGCAGACATTGCAGGAACGA GGCCAG

GGTCATCACTCTCCAATTGCAGCATACAATGAAGAAAAAGACTCTTTCCTGGTATTGGATGTCTCCAGATACAA GTATCC

ATCTTGGTGGgTACCAGTTTCAGAGCTGTTTGCAGCAGTCCTTCCAAGAGATCCGTCAGATGTAGAAGATCCAG GAAGGG

GCCTCGTGTTTGTCTGGGCTCAAACATCAGATGGGACACTGCAACCTTCCTTAGGAAGGGAGTTCTTCTCATCA GGGCTG

GACGATGGCATGCCTACTTATGACTTTTGCGATGATGTGCACTCAGCATTTGGTCTTGAGGTGCTGTCCTTTTG GCTGGG

ATTGGGATATTGTGCTTGCCTCTGTTGTTGTTTATGTCAACTGGAGTTATCAAGGTCAGCTACCAGGCACCCTC TTGGGG

GATCGTTGGAATAAGAAACTTCTGGGCTAGgGACCTGAGATGTTCAATCAAAGTGCAGGGGCATAAACAGATTG CTACCG

AATTCCATCAAGACCAGCAGCGAAGAGCAGgCGCTGACTATGAGTTATCTGCAACATCATTTGATGACACTGAA TTATCA

CCACTGTGCGCTGTTAGATGAACTGAAGCGGTTCTTCTGATTCCTTTGCATGTCAGCTTTTAACGTTCTTGACA AAACTG

TTCCTGATGTCATCATGCGTGCCCTCGGTTTTTACATGAGCACAAGCTCTTCGAATCATCAATGTAAAGAGTAC ATTGAA

TAGCATAGATAGGAAAACTCACAAAAATTGAACATATTTTTTGGATAATCAGGCTATCGAGAGCAACTGGTGGA AAAAGT

TATACATACTATCTCTGTCTCAATACAACAACATTTTTATGAAGAAACAGAAGCCTTGTAAACATAGAAAGCAG GAAATG

TTCATCATCTCCAATTAATTCTGAGGTCTGATGTAGCAAGCTTCACCAAAGAGGTAAGAACATGCAGTTCCAAG GATTCT

GTTCTGACCACTCTCCTGCTCAGGTTTTGATTTTCATGGCCTCTGCCACTGGAGAAGCGACAGCTCCGTTGTTA TTGCTG

GTAGCGTTCAGCTTGTTTGACACCTCATCAATGGAGGCGTTGAGCTGTGCAATCTTATCAAACAGATCCTGTTT GGTCTG

TTCAGGGTCCTTCACCTCATCCTCAAGAAATCTTGGAAGTTTCAGGCGTTGATCATCGCCCAAGAGCGCTTTAG AGATCT

GCAGAGAACAAAATAAAACCGCATGGTTCACACACCGCGGGAAAGAACGGCACATGGAATGAATGACATGGCAT 
GTTAAT

AAGCACAGTTAAATAAATCTCCATGTTGAGgTATCTCAATTTCAAGATTATGATGTAGGTAACTGCGTTTCAGC AGTTAT

TCAAAAAATTAGTAGGCCTACATGGCTATTGCACATGTGTATGTGATGCCTTTGCAATTCATCATACCTGCGGG GCAAAT

ATGAATCCCAGAGTACCGAAAACAACACCCCCAAGGACAAATCCAGAGACGAAGCTGTCACTTCGGTCACTATT CTCAGC

TCTGCAAGCGACAATAATGAGATCAACAACACAAATCCAATCATGAAGGTATGAGCATGTCTCTCACCTAGCTA CGATAT

TCCTCTCTTTTCTTAACGTTCTTGTCACAGGAGTATGCTGTATCGGAGAGCTTTCGCGACAATGGAAAGATCCC GAACAT

AAGTTCCCTGAGgTCAAACAAAAGATGGGAGGAGAATTCATCGGGGTACAAAACGTGCAGCTTACCTGTCCTGC TTGTTA

ACATTCTTGAACACTACTATGCGTATCGTTAATCAGGAGGACTCCGTGTAAGCGTTAAAGGGCAATCATGCAGC CACGTA AG

>comp11852_c0_seq2_len=2416

TTTGTTCACTTTTCGGCAGTAAATAAGCAATATTGTAGTCAAGTTGTTATCCTCGCTCTTACCTTTTGATTTCA AGGATG

CTTTCAGTCTTGCTTTGTACCAGAATTTTGAAAACATACATTCTCTTTCAAGTTCTGACCTCATCCGTCCAAGG GGAGTT

GGGTCTGCCTTGTTCCCTTTTGTCATTAACATCACACGAAGGACAAAAGGCCTTCATTCGTGCCAAGTATAAGG GCGATG

CATTTTCCATGCTGGAGAACTTTGTTGCACAGGTCAACGATGCGTACTGCGGGCCAGCCTCTGCTGTAACAGTC CTGAAT

TCTTTGGGGTGGGGCTCCCTATGCAGGACCCTGCTTCAGGGTTAGACTTGCCTGAATTTGCATATTACACACAA GACAAC

GTATTCTCACTAGAAACAGAAAGAGTGAAGCCCAAAGCTCAGATCAGGGAAGAGGGCCTCAGCCTATCAGAGCT CTCAAG

TTTCATCGCTGCTCACTCAGGAGTCAACGCCACGTACCGCCACAGCAGGGACATGAAAGACATGAATGAGTTTC GACAGC

TGGCTGTGTCAGCTCTAGCACAGCCTAATGTGTATGTTATTGTCAACTTCTTCCGGCAGACATTGCAGGAACGA GGCCAG

GGTCATCACTCTCCAATTGCAGCATACAATGAAGAAAAAGACTCTTTCCTGGTATTGGATGTCTCCAGATACAA GTATCC

ATCTTGGTGGGTACCAGTTTCAGAGCTGTTTGCAGCAGTCCTTCCAAGAGATCCGTCAGATGTAGAAGATCCAG GAAGGG

GCCTCGTGTTTGTCTGGGCTCAAACATCAGATGGGACACTGCAACCTTCCTTAGGAAGGGAGTTCTTCTCATCA GGGCTG

GACGATGGCATGCCTACTTATGACTTTTGCGATGATGTGCACTCAGCATTTGGTCTTGAGGTGCTGTCCTTTTG GCTGGG

ATTGGGATATTGTGCTTGCCTCTGTTGTTGTTTATGTCAACTGGAGTTATCAAGGTCAGCTACCAGGCACCCTC TTGGGG

GATCGTTGGAATAAGAAACTTCTGGGCTAGgGACCTGAGATGTTCAATCAAAGTGCAGGGGCATAAACAGATTG CTACCG

AATTCCATCAAGACCAGCAGCGAAGAGCAGGCGCTGACTATGAGTTATCTGCAACATCATTTGATGACACTGAA TTATCA

CCACTGTGCGCTGTTAGATGAACTGAAGCGGTTCTTCTGATTCCTTTGCATGTCAGCTTTTAACGTTCTTGACA AAACTG

TTCCTGATGTCATCATGCGTGCCCTCGGTTTTTACATGAGCACAAGCTCTTCGAATCATCAATGTAAAGAGTAC ATTGAA

TAGCATAGATAGGAAAACTCACAAAAATTGAACATATTTTTTGGATAATCAGGCTATCGAGAGCAACTGGTGGA AAAAGT

TATACATACTATCTCTGTCTCAATACAACAACATTTTTATGAAGAAACAGAAGCCTTGTAAACATAGAAAGCAG GAAATG

TTCATCATCTCCAATTAATTCTGAGGTCTGATGTAGCAAGCTTCACCAAAGAGGTAAGAACATGCAGTTCCAAG GATTCT 
GTTCTGACCACTCTCCTGCTCAGGTTTTGATTTTCATGGCCTCTGCCACTGGAGAAGCGACAGCTCCGTTGTTA TTGCTG

GTAGCGTTCAGCTTGTTTGACACCTCATCAATGGAGGCGTTGAGCTGTGCAATCTTATCAAACAGATCCTGTTT GGTCTG

TTCAGGGTCCTTCACCTCATCCTCAAGAAATCTTGGAAGTTTCAGGCGTTGATCATCGCCCAAGAGCGCTTTAG AGATCT

GCAGAGAACAAAATAAAACCGCATGGTTCACACACCGCGGGAAAGAACGGCACATGGAATGAATGACATGGCAT GTTAAT

AAGCACAGTTAAATAAATCTCCATGTTGAGgTATCTCAATTTCAAGATTATGATGTAGgTAACTGCGTTTCAGC AGTTAT

TCAAAAAATTAGTAGGCCTACATGGCTATTGCACATGTGTATGTGATGCCTTTGCAATTCATCATACCTGCGGG GCAAAT

ATGAATCCCAGAGTACCGAAAACAACACCCCCAAGGACAAATCCAGAGACGAAGCTGTCACTTCGGTCACTATT CTCAGC

TCTAGCTACGATATTCCTCTCTTTTCTTAACGTTCTTGTCACAGGAGTATGCTGTATCGGAGAGCTTTCGCGAC AATGGA

AAGATCCCGAACATAAGTTCCCTGAGgTCAAACAAAAGATGGGAGGAGAATTCATCGGGgTACAAAACGTGCAG CTTACC

TGTCCTGCTTGTTAACATTCTTGAACACTACTATGCGTATCGTTAATCAGGAGGACTCCGTGTAAGCGTTAAAG GGCAAT CATGCAGCCACGTAAG

>comp11852_c0_seq3_len=2253

TTTGTTCACTTTTCGgCAGTAAATAAGCAATATTGTAGTCAAGTTGTTATCCTCGCTCTTACCTTTTGATTTCA AGGATG

CTTTCAGTCTTGCTTTGTACCAGAATTTTGAAAACATACATTCTCTTTCAAGTTCTGACCTCATCCGTCCAAGG GGAGTT

GGGTCTGCCTTGTTCCCTTTTGTCATTAACATCACACGAAGGACAAAAGGCCTTCATTCGTGCCAAGTATAAGG GCGATG

CATTTTCCATGCTGGAGAACTTTGTTGCACAGGTCAACGATGCGTACTGCGGGCCAGCCTCTGCTGTAACAGTC CTGAAT

TCTTTGGGGTGGGGCTCCCTATGCAGGACCCTGCTTCAGGGTTAGACTTGCCTGAATTTGCATATTACACACAA GACAAC

GTATTCTCACTAGAAACAGAAAGAGTGAAGCCCAAAGCTCAGATCAGGGAAGAGGGCCTCAGCCTATCAGAGCT

CTCAAG

TTTCATCGCTGCTCACTCAGGAGTCAACGCCACGTACCGCCACAGCAGGGACATGAAAGACATGAATGAGTTTC GACAGC

TGGCTGTGTCAGCTCTAGCACAGCCTAATGTGTATGTTATTGTCAACTTCTTCCGGCAGACATTGCAGGAACGA GGCCAG

GGTCATCACTCTCCAATTGCAGCATACAATGAAGAAAAAGACTCTTTCCTGGTATTGGATGTCTCCAGATACAA GTATCC

ATCTTGGTGGGTACCAGTTTCAGAGCTGTTTGCAGCAGTCCTTCCAAGAGATCCGTCAGATGTAGAAGATCCAG GAAGGG

GCCTCGTGTTTGTCTGGGCTCAAACATCAGATGGGACACTGCAACCTTCCTTAGGAAGGGAGTTCTTCTCATCA GGGCTG

GACGATGGCATGCCTACTTATGACTTTTGCGATGATGTGCACTCAGCATTTGGTCTTGAGGTGCTGTCCTTTTG GCTGGG

ATTGGGATATTGTGCTTGCCTCTGTTGTTGTTTATGTCAACTGGAGTTATCAAGGTCAGCTACCAGGCACCCTC TTGGGG

GATCGTTGGAATAAGAAACTTCTGGGCTAGGGACCTGAGATGTTCAATCAAAGTGCAGGGGCATAAACAGATTG CTACCG

AATTCCATCAAGACCAGCAGCGAAGAGCAGGCGCTGACTATGAGTTATCTGCAACATCATTTGATGACACTGAA TTATCA

CCACTGTGCGCTGTTAGATGAACTGAAGCGGTTCTTCTGATTCCTTTGCATGTCAGCTTTTAACGTTCTTGACA AAACTG

TTCCTGATGTCATCATGCGTGCCCTCGgTTTTTACATGAGCACAAGCTCTTCGAATCATCAATGTAAAGAGTAC ATTGAA

TAGCATAGATAGGAAAACTCACAAAAATTGAACATATTTTTTGGATAATCAGGCTATCGAGAGCAACTGGTGGA 
AAAAGT

TATACATACTATCTCTGTCTCAATACAACAACATTTTTATGAAGAAACAGAAGCCTTGTAAACATAGAAAGCAG GAAATG

TTCATCATCTCCAATTAATTCTGAGGTCTGATGTAGCAAGCTTCACCAAAGAGGTAAGAACATGCAGTTCCAAG GATTCT

GTTCTGACCACTCTCCTGCTCAGGTTTTGATTTTCATGGCCTCTGCCACTGGAGAAGCGACAGCTCCGTTGTTA TTGCTG

GTAGCGTTCAGCTTGTTTGACACCTCATCAATGGAGGCGTTGAGCTGTGCAATCTTATCAAACAGATCCTGTTT GGTCTG

TTCAGGGTCCTTCACCTCATCCTCAAGAAATCTTGGAAGTTTCAGGCGTTGATCATCGCCCAAGAGCGCTTTAG AGATCT

GCGGGGCAAATATGAATCCCAGAGTACCGAAAACAACACCCCCAAGGACAAATCCAGAGACGAAGCTGTCACTT CGGTCA

CTATTCTCAGCTCTGCAAGCGACAATAATGAGATCAACAACACAAATCCAATCATGAAGGTATGAGCATGTCTC TCACCT

AGCTACGATATTCCTCTCTTTTCTTAACGTTCTTGTCACAGGAGTATGCTGTATCGGAGAGCTTTCGCGACAAT GGAAAG

ATCCCGAACATAAGTTCCCTGAGGTCAAACAAAAGATGGGAGGAGAATTCATCGGGGTACAAAACGTGCAGCTT ACCTGT

CCTGCTTGTTAACATTCTTGAACACTACTATGCGTATCGTTAATCAGGAGGACTCCGTGTAAGCGTTAAAGGGC AATCAT GCAGCCACGTAAG

>comp11852_c0_seq4_len=2187

TTTGTTCACTTTTCGGCAGTAAATAAGCAATATTGTAGTCAAGTTGTTATCCTCGCTCTTACCTTTTGATTTCA AGGATG

CTTTCAGTCTTGCTTTGTACCAGAATTTTGAAAACATACATTCTCTTTCAAGTTCTGACCTCATCCGTCCAAGG GGAGTT

GGGTCTGCCTTGTTCCCTTTTGTCATTAACATCACACGAAGGACAAAAGGCCTTCATTCGTGCCAAGTATAAGG GCGATG

CATTTTCCATGCTGGAGAACTTTGTTGCACAGGTCAACGATGCGTACTGCGGGCCAGCCTCTGCTGTAACAGTC CTGAAT

TCTTTGGGGTGGGGCTCCCTATGCAGGACCCTGCTTCAGGGTTAGACTTGCCTGAATTTGCATATTACACACAA GACAAC

GTATTCTCACTAGAAACAGAAAGAGTGAAGCCCAAAGCTCAGATCAGGGAAGAGGGCCTCAGCCTATCAGAGCT

CTCAAG

TTTCATCGCTGCTCACTCAGGAGTCAACGCCACGTACCGCCACAGCAGGGACATGAAAGACATGAATGAGTTTC GACAGC

TGGCTGTGTCAGCTCTAGCACAGCCTAATGTGTATGTTATTGTCAACTTCTTCCGGCAGACATTGCAGGAACGA GGCCAG

GGTCATCACTCTCCAATTGCAGCATACAATGAAGAAAAAGACTCTTTCCTGGTATTGGATGTCTCCAGATACAA GTATCC

ATCTTGGTGGGTACCAGTTTCAGAGCTGTTTGCAGCAGTCCTTCCAAGAGATCCGTCAGATGTAGAAGATCCAG GAAGGG

GCCTCGTGTTTGTCTGGGCTCAAACATCAGATGGGACACTGCAACCTTCCTTAGGAAGGGAGTTCTTCTCATCA GGGCTG

GACGATGGCATGCCTACTTATGACTTTTGCGATGATGTGCACTCAGCATTTGGTCTTGAGGTGCTGTCCTTTTG GCTGGG

ATTGGGATATTGTGCTTGCCTCTGTTGTTGTTTATGTCAACTGGAGTTATCAAGGTCAGCTACCAGGCACCCTC TTGGGG

GATCGTTGGAATAAGAAACTTCTGGGCTAGgGACCTGAGATGTTCAATCAAAGTGCAGGgGCATAAACAGATTG CTACCG

AATTCCATCAAGACCAGCAGCGAAGAGCAGGCGCTGACTATGAGTTATCTGCAACATCATTTGATGACACTGAA TTATCA

CCACTGTGCGCTGTTAGATGAACTGAAGCGGTTCTTCTGATTCCTTTGCATGTCAGCTTTTAACGTTCTTGACA AAACTG

TTCCTGATGTCATCATGCGTGCCCTCGGTTTTTACATGAGCACAAGCTCTTCGAATCATCAATGTAAAGAGTAC ATTGAA 
TAGCATAGATAgGAAAACTCACAAAAATTGAACATATTTTTTGGATAATCAGgCTATCGAGAGCAACTGgTGGA AAAAGT

TATACATACTATCTCTGTCTCAATACAACAACATTTTTATGAAGAAACAGAAGCCTTGTAAACATAGAAAGCAG GAAATG

TTCATCATCTCCAATTAATTCTGAGgTCTGATGTAGCAAGCTTCACCAAAGAGgTAAGAACATGCAGTTCCAAG GATTCT

GTTCTGACCACTCTCCTGCTCAGGTTTTGATTTTCATGGCCTCTGCCACTGGAGAAGCGACAGCTCCGTTGTTA TTGCTG

GTAGCGTTCAGCTTGTTTGACACCTCATCAATGGAGGCGTTGAGCTGTGCAATCTTATCAAACAGATCCTGTTT GGTCTG

TTCAGGGTCCTTCACCTCATCCTCAAGAAATCTTGGAAGTTTCAGGCGTTGATCATCGCCCAAGAGCGCTTTAG AGATCT

GCGGGGCAAATATGAATCCCAGAGTACCGAAAACAACACCCCCAAGGACAAATCCAGAGACGAAGCTGTCACTT CGGTCA

CTATTCTCAGCTCTAGCTACGATATTCCTCTCTTTTCTTAACGTTCTTGTCACAGGAGTATGCTGTATCGGAGA GCTTTC

GCGACAATGGAAAGATCCCGAACATAAGTTCCCTGAGGTCAAACAAAAGATGGGAGgAGAATTCATCGGGGTAC AAAACG

TGCAGCTTACCTGTCCTGCTTGTTAACATTCTTGAACACTACTATGCGTATCGTTAATCAGGAGGACTCCGTGT AAGCGT TAAAGGGCAATCATGCAGCCACGTAAG

>comp11852_c0_seq5_len=2129

TTTGTTCACTTTTCGGCAGTAAATAAGCAATATTGTAGTCAAGTTGTTATCCTCGCTCTTACCTTTTGATTTCA AGGATG

CTTTCAGTCTTGCTTTGTACCAGAATTTTGAAAACATACATTCTCTTTCAAGTTCTGACCTCATCCGTCCAAGg GGAGTT

GGGTCTGCCTTGTTCCCTTTTGTCATTAACATCACACGAAGGACAAAAGGCCTTCATTCGTGCCAAGTATAAGG GCGATG

CATTTTCCATGCTGGAGAACTTTGTTGCACAGGTCAACGATGCGTACTGCGGGCCAGCCTCTGCTGTAACAGTC CTGAAT

TCTTTGGGGTGGGGCTCCCTATGCAGGACCCTGCTTCAGGGTTAGACTTGCCTGAATTTGCATATTACACACAA GACAAC

GTATTCTCACTAGAAACAGAAAGAGTGAAGCCCAAAGCTCAGATCAGGGAAGAGGGCCTCAGCCTATCAGAGCT CTCAAG

TTTCATCGCTGCTCACTCAGGAGTCAACGCCACGTACCGCCACAGCAGGGACATGAAAGACATGAATGAGTTTC GACAGC

TGGCTGTGTCAGCTCTAGCACAGCCTAATGTGTATGTTATTGTCAACTTCTTCCGGCAGACATTGCAGGAACGA GGCCAG

GGTCATCACTCTCCAATTGCAGCATACAATGAAGAAAAAGACTCTTTCCTGGTATTGGATGTCTCCAGATACAA GTATCC

ATCTTGGTGGGTACCAGTTTCAGAGCTGTTTGCAGCAGTCCTTCCAAGAGATCCGTCAGATGTAGAAGATCCAG GAAGGG

GCCTCGTGTTTGTCTGGGCTCAAACATCAGATGGGACACTGCAACCTTCCTTAGGAAGGGAGTTCTTCTCATCA GGGCTG

GACGATGGCATGCCTACTTATGACTTTTGCGATGATGTGCACTCAGCATTTGGTCTTGAGGTGCTGTCCTTTTG GCTGGG

ATTGGGATATTGTGCTTGCCTCTGTTGTTGTTTATGTCAACTGGAGTTATCAAGGTCAGCTACCAGGCACCCTC TTGGGG

GATCGTTGGAATAAGAAACTTCTGGGCTAGGGACCTGAGATGTTCAATCAAAGTGCAGGGGCATAAACAGATTG CTACCG

AATTCCATCAAGACCAGCAGCGAAGAGCAGGCGCTGACTATGAGTTATCTGCAACATCATTTGATGACACTGAA TTATCA

CCACTGTGCGCTGTTAGATGAACTGAAGCGGTTCTTCTGATTCCTTTGCATGTCAGCTTTTAACGTTCTTGACA AAACTG

TTCCTGATGTCATCATGCGTGCCCTCGgTTTTTACATGAGCACAAGCTCTTCGAATCATCAATGTAAAGAGTAC ATTGAA

TAGCATAGATAGgAAAACTCACAAAAATTGAACATATTTTTTGGATAATCAGgCTATCGAGAGCAACTGGTGGA 
AAAAGT

TATACATACTATCTCTGTCTCAATACAACAACATTTTTATGAAGAAACAGAAGCCTTGTAAACATAGAAAGCAG GAAATG

TTCATCATCTCCAATTAATTCTGAGGTCTGATGTAGCAAGCTTCACCAAAGAGGTAAGAACATGCAGTTCCAAG GATTCT

GTTCTGACCACTCTCCTGCTCAGGTTTTGATTTTCATGGCCTCTGCCACTGGAGAAGCGACAGCTCCGTTGTTA TTGCTG

GTAGCGTTCAGCTTGTTTGACACCTCATCAATGGAGGCGTTGAGCTGTGCAATCTTATCAAACAGATCCTGTTT GGTCTG

TTCAGGGTCCTTCACCTCATCCTCAAGAAATCTTGGAAGTTTCAGGCGTTGATCATCGCCCAAGAGCGCTTTAG AGATCT

GCGGGGCAAATATGAATCCCAGAGTACCGAAAACAACACCCCCAAGGACAAATCCAGAGACGAAGCTGTCACTT CGGTCA

CTATTCTCAGCTCTAGCTACGATATTCCTCTCTTTTCTTAACGTTCTTGTCACAGGAGTATGCTGTATCGGAGA GCTTTC

GCGACAATGGAAAGATCCCGAACATAAGTTCCCTGTCCTGCTTGTTAACATTCTTGAACACTACTATGCGTATC GTTAAT CAGGAGGACTCCGTGTAAGCGTTAAAGGGCAATCATGCAGCCACGTAAG

>comp11852_c0_seq6_len=2424

TTTGTTCACTTTTCGGCAGTAAATAAGCAATATTGTAGTCAAGTTGTTATCCTCGCTCTTACCTTTTGATTTCA AGGATG

CTTTCAGTCTTGCTTTGTACCAGAATTTTGAAAACATACATTCTCTTTCAAGTTCTGACCTCATCCGTCCAAGG GGAGTT

GGGTCTGCCTTGTTCCCTTTTGTCATTAACATCACACGAAGGACAAAAGGCCTTCATTCGTGCCAAGTATAAGg GCGATG

CATTTTCCATGCTGGAGAACTTTGTTGCACAGGTCAACGATGCGTACTGCGGGCCAGCCTCTGCTGTAACAGTC CTGAAT

TCTTTGGGGTGGGGCTCCCTATGCAGGACCCTGCTTCAGGGTTAGACTTGCCTGAATTTGCATATTACACACAA GACAAC

GTATTCTCACTAGAAACAGAAAGAGTGAAGCCCAAAGCTCAGATCAGGGAAGAGGGCCTCAGCCTATCAGAGCT CTCAAG

TTTCATCGCTGCTCACTCAGGAGTCAACGCCACGTACCGCCACAGCAGGGACATGAAAGACATGAATGAGTTTC GACAGC

TGGCTGTGTCAGCTCTAGCACAGCCTAATGTGTATGTTATTGTCAACTTCTTCCGGCAGACATTGCAGGAACGA GGCCAG

GGTCATCACTCTCCAATTGCAGCATACAATGAAGAAAAAGACTCTTTCCTGGTATTGGATGTCTCCAGATACAA GTATCC

ATCTTGGTGGGTACCAGTTTCAGAGCTGTTTGCAGCAGTCCTTCCAAGAGATCCGTCAGATGTAGAAGATCCAG GAAGGG

GCCTCGTGTTTGTCTGGGCTCAAACATCAGATGGGACACTGCAACCTTCCTTAGGAAGGGAGTTCTTCTCATCA GGGCTG

GACGATGGCATGCCTACTTATGACTTTTGCGATGATGTGCACTCAGCATTTGGTCTTGAGGTGCTGTCCTTTTG GCTGGG

ATTGGGATATTGTGCTTGCCTCTGTTGTTGTTTATGTCAACTGGAGTTATCAAGGTCAGCTACCAGGCACCCTC TTGGGG

GATCGTTGGAATAAGAAACTTCTGGGCTAGGGACCTGAGATGTTCAATCAAAGTGCAGGGGCATAAACAGATTG CTACCG

AATTCCATCAAGACCAGCAGCGAAGAGCAGGCGCTGACTATGAGTTATCTGCAACATCATTTGATGACACTGAA TTATCA

CCACTGTGCGCTGTTAGATGAACTGAAGCGGTTCTTCTGATTCCTTTGCATGTCAGCTTTTAACGTTCTTGACA AAACTG

TTCCTGATGTCATCATGCGTGCCCTCGGTTTTTACATGAGCACAAGCTCTTCGAATCATCAATGTAAAGAGTAC ATTGAA

TAGCATAGATAGGAAAACTCACAAAAATTGAACATATTTTTTGGATAATCAGGCTATCGAGAGCAACTGGTGGA AAAAGT

TATACATACTATCTCTGTCTCAATACAACAACATTTTTATGAAGAAACAGAAGCCTTGTAAACATAGAAAGCAG GAAATG 
TTCATCATCTCCAATTAATTCTGAGGTCTGATGTAGCAAGCTTCACCAAAGAGGTAAGAACATGCAGTTCCAAG GATTCT

GTTCTGACCACTCTCCTGCTCAGGTTTTGATTTTCATGGCCTCTGCCACTGGAGAAGCGACAGCTCCGTTGTTA TTGCTG

GTAGCGTTCAGCTTGTTTGACACCTCATCAATGGAGGCGTTGAGCTGTGCAATCTTATCAAACAGATCCTGTTT GGTCTG

TTCAGGGTCCTTCACCTCATCCTCAAGAAATCTTGGAAGTTTCAGGCGTTGATCATCGCCCAAGAGCGCTTTAG AGATCT

GCAGAGAACAAAATAAAACCGCATGGTTCACACACCGCGGGAAAGAACGGCACATGGAATGAATGACATGGCAT GTTAAT

AAGCACAGTTAAATAAATCTCCATGTTGAGgTATCTCAATTTCAAGATTATGATGTAGgTAACTGCGTTTCAGC AGTTAT

TCAAAAAATTAGTAGGCCTACATGGCTATTGCACATGTGTATGTGATGCCTTTGCAATTCATCATACCTGCGGG GCAAAT

ATGAATCCCAGAGTACCGAAAACAACACCCCCAAGGACAAATCCAGAGACGAAGCTGTCACTTCGGTCACTATT CTCAGC

TCTGCAAGCGACAATAATGAGATCAACAACACAAATCCAATCATGAAGGTATGAGCATGTCTCTCACCTAGCTA CGATAT

TCCTCTCTTTTCTTAACGTTCTTGTCACAGGAGTATGCTGTATCGGAGAGCTTTCGCGACAATGGAAAGATCCC GAACAT

AAGTTCCCTGTCCTGCTTGTTAACATTCTTGAACACTACTATGCGTATCGTTAATCAGGAGGACTCCGTGTAAG CGTTAA AGGGCAATCATGCAGCCACGTAAG

>comp11852_c0_seq7_len=2195

TTTGTTCACTTTTCGGCAGTAAATAAGCAATATTGTAGTCAAGTTGTTATCCTCGCTCTTACCTTTTGATTTCA AGGATG

CTTTCAGTCTTGCTTTGTACCAGAATTTTGAAAACATACATTCTCTTTCAAGTTCTGACCTCATCCGTCCAAGg GGAGTT

GGGTCTGCCTTGTTCCCTTTTGTCATTAACATCACACGAAGGACAAAAGGCCTTCATTCGTGCCAAGTATAAGG GCGATG

CATTTTCCATGCTGGAGAACTTTGTTGCACAGGTCAACGATGCGTACTGCGGGCCAGCCTCTGCTGTAACAGTC CTGAAT

TCTTTGGGGTGGGGCTCCCTATGCAGGACCCTGCTTCAGGGTTAGACTTGCCTGAATTTGCATATTACACACAA GACAAC

GTATTCTCACTAGAAACAGAAAGAGTGAAGCCCAAAGCTCAGATCAGGGAAGAGGGCCTCAGCCTATCAGAGCT CTCAAG

TTTCATCGCTGCTCACTCAGGAGTCAACGCCACGTACCGCCACAGCAGGGACATGAAAGACATGAATGAGTTTC GACAGC

TGGCTGTGTCAGCTCTAGCACAGCCTAATGTGTATGTTATTGTCAACTTCTTCCGGCAGACATTGCAGGAACGA GGCCAG

GGTCATCACTCTCCAATTGCAGCATACAATGAAGAAAAAGACTCTTTCCTGGTATTGGATGTCTCCAGATACAA GTATCC

ATCTTGGTGGGTACCAGTTTCAGAGCTGTTTGCAGCAGTCCTTCCAAGAGATCCGTCAGATGTAGAAGATCCAG GAAGGG

GCCTCGTGTTTGTCTGGGCTCAAACATCAGATGGGACACTGCAACCTTCCTTAGGAAGGGAGTTCTTCTCATCA GGGCTG

GACGATGGCATGCCTACTTATGACTTTTGCGATGATGTGCACTCAGCATTTGGTCTTGAGGTGCTGTCCTTTTG GCTGGG

ATTGGGATATTGTGCTTGCCTCTGTTGTTGTTTATGTCAACTGGAGTTATCAAGGTCAGCTACCAGGCACCCTC TTGGGG

GATCGTTGGAATAAGAAACTTCTGGGCTAGgGACCTGAGATGTTCAATCAAAGTGCAGGGGCATAAACAGATTG CTACCG

AATTCCATCAAGACCAGCAGCGAAGAGCAGGCGCTGACTATGAGTTATCTGCAACATCATTTGATGACACTGAA TTATCA

CCACTGTGCGCTGTTAGATGAACTGAAGCGGTTCTTCTGATTCCTTTGCATGTCAGCTTTTAACGTTCTTGACA AAACTG

TTCCTGATGTCATCATGCGTGCCCTCGGTTTTTACATGAGCACAAGCTCTTCGAATCATCAATGTAAAGAGTAC 
ATTGAA

TAGCATAGATAGgAAAACTCACAAAAATTGAACATATTTTTTGGATAATCAGGCTATCGAGAGCAACTGGTGGA AAAAGT

TATACATACTATCTCTGTCTCAATACAACAACATTTTTATGAAGAAACAGAAGCCTTGTAAACATAGAAAGCAG GAAATG

TTCATCATCTCCAATTAATTCTGAGgTCTGATGTAGCAAGCTTCACCAAAGAGGTAAGAACATGCAGTTCCAAG GATTCT

GTTCTGACCACTCTCCTGCTCAGGTTTTGATTTTCATGGCCTCTGCCACTGGAGAAGCGACAGCTCCGTTGTTA TTGCTG

GTAGCGTTCAGCTTGTTTGACACCTCATCAATGGAGGCGTTGAGCTGTGCAATCTTATCAAACAGATCCTGTTT GGTCTG

TTCAGGGTCCTTCACCTCATCCTCAAGAAATCTTGGAAGTTTCAGGCGTTGATCATCGCCCAAGAGCGCTTTAG AGATCT

GCGGGGCAAATATGAATCCCAGAGTACCGAAAACAACACCCCCAAGGACAAATCCAGAGACGAAGCTGTCACTT CGGTCA

CTATTCTCAGCTCTGCAAGCGACAATAATGAGATCAACAACACAAATCCAATCATGAAGgTATGAGCATGTCTC

TCACCT

AGCTACGATATTCCTCTCTTTTCTTAACGTTCTTGTCACAGGAGTATGCTGTATCGGAGAGCTTTCGCGACAAT GGAAAG

ATCCCGAACATAAGTTCCCTGTCCTGCTTGTTAACATTCTTGAACACTACTATGCGTATCGTTAATCAGGAGGA

CTCCGT GTAAGCGTTAAAGGGCAATCATGCAGCCACGTAAG

>comp11852_c0_seq8_len=2358

TTTGTTCACTTTTCGgCAGTAAATAAGCAATATTGTAGTCAAGTTGTTATCCTCGCTCTTACCTTTTGATTTCA AGGATG

CTTTCAGTCTTGCTTTGTACCAGAATTTTGAAAACATACATTCTCTTTCAAGTTCTGACCTCATCCGTCCAAGG GGAGTT

GGGTCTGCCTTGTTCCCTTTTGTCATTAACATCACACGAAGGACAAAAGGCCTTCATTCGTGCCAAGTATAAGG GCGATG

CATTTTCCATGCTGGAGAACTTTGTTGCACAGGTCAACGATGCGTACTGCGGGCCAGCCTCTGCTGTAACAGTC CTGAAT

TCTTTGGGGTGGGGCTCCCTATGCAGGACCCTGCTTCAGGGTTAGACTTGCCTGAATTTGCATATTACACACAA GACAAC

GTATTCTCACTAGAAACAGAAAGAGTGAAGCCCAAAGCTCAGATCAGGGAAGAGGGCCTCAGCCTATCAGAGCT

CTCAAG

TTTCATCGCTGCTCACTCAGGAGTCAACGCCACGTACCGCCACAGCAGGGACATGAAAGACATGAATGAGTTTC GACAGC

TGGCTGTGTCAGCTCTAGCACAGCCTAATGTGTATGTTATTGTCAACTTCTTCCGGCAGACATTGCAGGAACGA GGCCAG

GGTCATCACTCTCCAATTGCAGCATACAATGAAGAAAAAGACTCTTTCCTGGTATTGGATGTCTCCAGATACAA GTATCC

ATCTTGGTGGGTACCAGTTTCAGAGCTGTTTGCAGCAGTCCTTCCAAGAGATCCGTCAGATGTAGAAGATCCAG GAAGGG

GCCTCGTGTTTGTCTGGGCTCAAACATCAGATGGGACACTGCAACCTTCCTTAGGAAGGGAGTTCTTCTCATCA GGGCTG

GACGATGGCATGCCTACTTATGACTTTTGCGATGATGTGCACTCAGCATTTGGTCTTGAGGTGCTGTCCTTTTG GCTGGG

ATTGGGATATTGTGCTTGCCTCTGTTGTTGTTTATGTCAACTGGAGTTATCAAGGTCAGCTACCAGGCACCCTC TTGGGG

GATCGTTGGAATAAGAAACTTCTGGGCTAGgGACCTGAGATGTTCAATCAAAGTGCAGGgGCATAAACAGATTG CTACCG

AATTCCATCAAGACCAGCAGCGAAGAGCAGGCGCTGACTATGAGTTATCTGCAACATCATTTGATGACACTGAA TTATCA

CCACTGTGCGCTGTTAGATGAACTGAAGCGGTTCTTCTGATTCCTTTGCATGTCAGCTTTTAACGTTCTTGACA AAACTG

TTCCTGATGTCATCATGCGTGCCCTCGGTTTTTACATGAGCACAAGCTCTTCGAATCATCAATGTAAAGAGTAC ATTGAA 
TAGCATAGATAGgAAAACTCACAAAAATTGAACATATTTTTTGGATAATCAGgCTATCGAGAGCAACTGGTGGA AAAAGT

TATACATACTATCTCTGTCTCAATACAACAACATTTTTATGAAGAAACAGAAGCCTTGTAAACATAGAAAGCAG GAAATG

TTCATCATCTCCAATTAATTCTGAGGTCTGATGTAGCAAGCTTCACCAAAGAGGTAAGAACATGCAGTTCCAAG GATTCT

GTTCTGACCACTCTCCTGCTCAGGTTTTGATTTTCATGGCCTCTGCCACTGGAGAAGCGACAGCTCCGTTGTTA TTGCTG

GTAGCGTTCAGCTTGTTTGACACCTCATCAATGGAGGCGTTGAGCTGTGCAATCTTATCAAACAGATCCTGTTT GGTCTG

TTCAGGGTCCTTCACCTCATCCTCAAGAAATCTTGGAAGTTTCAGGCGTTGATCATCGCCCAAGAGCGCTTTAG AGATCT

GCAGAGAACAAAATAAAACCGCATGGTTCACACACCGCGGGAAAGAACGGCACATGGAATGAATGACATGGCAT GTTAAT

AAGCACAGTTAAATAAATCTCCATGTTGAGGTATCTCAATTTCAAGATTATGATGTAGgTAACTGCGTTTCAGC AGTTAT

TCAAAAAATTAGTAGGCCTACATGGCTATTGCACATGTGTATGTGATGCCTTTGCAATTCATCATACCTGCGGG GCAAAT

ATGAATCCCAGAGTACCGAAAACAACACCCCCAAGGACAAATCCAGAGACGAAGCTGTCACTTCGGTCACTATT CTCAGC

TCTAGCTACGATATTCCTCTCTTTTCTTAACGTTCTTGTCACAGGAGTATGCTGTATCGGAGAGCTTTCGCGAC AATGGA

AAGATCCCGAACATAAGTTCCCTGTCCTGCTTGTTAACATTCTTGAACACTACTATGCGTATCGTTAATCAGGA GGACTC CGTGTAAGCGTTAAAGGGCAATCATGCAGCCACGTAAG 
Horizontal gene transfer of phytochelatin synthases from bacteria to extremophilic green algae; Microbial Ecology; Olsson S*, Penacho V, Puente-Sánchez F, Díaz S, Aguilera A; * corresponding author, Department of Agricultural Sciences, P.O. Box 27, 00014 University of Helsinki, Finland, sanna.olsson@helsinki.fi

Online Resource 8 PCR products amplified in Dunaliella acidophila strain RT5 using gradient annealing run on $1 \%$ agarose gel (1X TBE). The gel electrophoresis shows amplification of products sized $1198 \mathrm{bp}$ and $542 \mathrm{bp}$ and several non-specific amplification products. The intensity of the nonspecific bands depends on the used annealing temperature and PCR program. Wells 1: Ladder $1 \mathrm{~kb}$ (Promega), marker sizes from $250 \mathrm{~kb}$ to $10000 \mathrm{~kb}$, well2: annealing $52-55^{\circ} \mathrm{C}$. Well 3: annealing 55$60^{\circ} \mathrm{C}$.

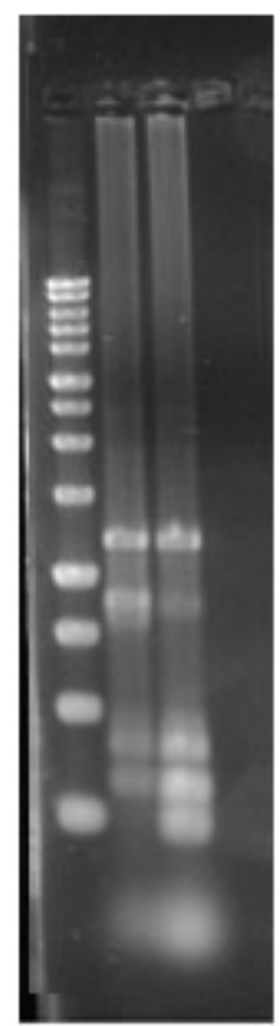


Horizontal gene transfer of phytochelatin synthases from bacteria to extremophilic green algae; Microbial Ecology; Olsson S*, Penacho V, Puente-Sánchez F, Díaz S, Aguilera A; * corresponding author, Department of Agricultural Sciences, P.O. Box 27, 00014 University of Helsinki, Finland, sanna.olsson@helsinki.fi

Online Resource 9 Phylogram based on the novel phytochelatin synthase genes and the most similar genes obtained with a BLASTx search against NCBI's databases. The tree represents the majority consensus of trees sampled after stationarity in the Bayesian analysis. PP values from the Bayesian inference $>0.50$ are indicated above each branch. The amino acid alignment was 383 aa long. 
$\$ 1$ Coleofasciculus chthonoplastes

Cyanobacteria

Cyanobacteria

Cyanobacteria

Cyanothece sp1

- Cyanothece sp3

\section{- Cyanothece sp2}

Microcoleus vaginatus

. 69 Chamaesiphon minutus

- Crinalium epipsammum

- Chroococcidiopsis thermalis

- Calothrix $s p$

[ Nostoc PCC 7107

764 Anabaena variabilis

$0.7 p 44-$ Anabaena sp

0.782 Cylindrospermum stagnale

Nostoc punctiforme

1 - Prochlorococcus marinus

d.906 Synechococcus sp1

- Cyanobium gracile

- Methylosinus trichosporium

${ }_{1}$ Burkholderia oklahomensis

0.998 Burkholderia pseudomallei1

0.860

Burkholderia pseudomallei2

$0995 \quad$ Aureococcus anophagefferens2

$0.985-$ Burkholderia xenovorans

$\begin{array}{ll}0.9 \$ 55 & \text { Burkholder } \\ 1 \text { CaPCS2 }\end{array}$

$\begin{array}{ll}1 & 1 \\ 1 & \text { DaPCS1 }\end{array}$

DaPCS1

0.829 Monosiga brevicollis

Coxiella burnetii

0.500 Francisella sp2

Saccharophagus degradans

Strongylocentrotus purpuratus

0.601 Thalassiosira oceanica

0.844 Polynucleobacter necessarius

1 - Rhodobacterales bacterium

Thalassosira profundimaris

$0.576 \quad 1 \quad$ Shewanella sediminis

1 Pantoea stewarti

1 Erwinia amylovora

Erwinia pyrifoliae

0.929 Aureococcus anophagefferens1

$0.505\left[\begin{array}{c}0.929 \\ 0.95\end{array}\right.$

- Thalassiosira pseudonana

- Rhizopus delemar

1 - Melampsora laricipopulina

- Puccinia graminis

0,095 Naegleria gruberi2

- Schizosaccharomyces japonicus

Cyanidioschyzon merolae

0.98

Schistosoma mansoni

1 - Albugo laibachii

0.983 [ Phytophthora infestans

Phytophthora sojae

1 - Nematostella vectensis 3

0.609 Nematostella vectensis2

0.954 Naegleria gruberi1

054694 1 1 Brugia malayi

1 Wuchereria bancrofti

1. Caenorhabditis elegans1

Caenorhabditis remanei

Volvox carteri1

$0.875-$ Chlorella variabilis

0.899 Coccomyxa subellipsoidea

0.998 - CaPCS1

1. Chlamydomonas reinhardtii

Volvox carteri2

0.993 - Pteris vittata

- Selaginella moellendorffii

${ }_{1}$ Lotus japonicus LjPCS3

Lotus japonicus LjPCS2 $7 R$

0.952 [ Ceratophyllum demersum

Salix

0.9451 Lathyrus sativus

0.671 [L Lotus japonicus

0.928 Avicennia germinans

0.9562 Arabidopsis thaliana

$\left[\begin{array}{l}\text { Arabidopsis thaliana } \\ \text { Hirschfeldia incana }\end{array}\right.$

Aegilops tauschi

Oryza sativa2

0.5 .5090 - Cynodon dactylon

L Oryza sativa1

0.962 Hordeum vulgare

0.61 Triticum aestivum

Triticum urartu

Cyanobacteria

Cyanobacteria

Cyanobacteria

Cyanobacteria

Bacteria

Chromalveolata

Bacteria

Green algae

Choanoflagellate

Bacteria

Bacteria

Bacteria

Metazoa

Chromalveolata

Bacteria

Bacteria

Diatom

Bacteria

Chromalveolata

Jungi

Fungi

Excavata

Fungi

Red algae

Metazoa

Fungi

Metazoa

Green algae

Streptophyta

Land plants 